\title{
THE EVOLUTION OF LIFE
}

H.CHARLTON BASTIAN 


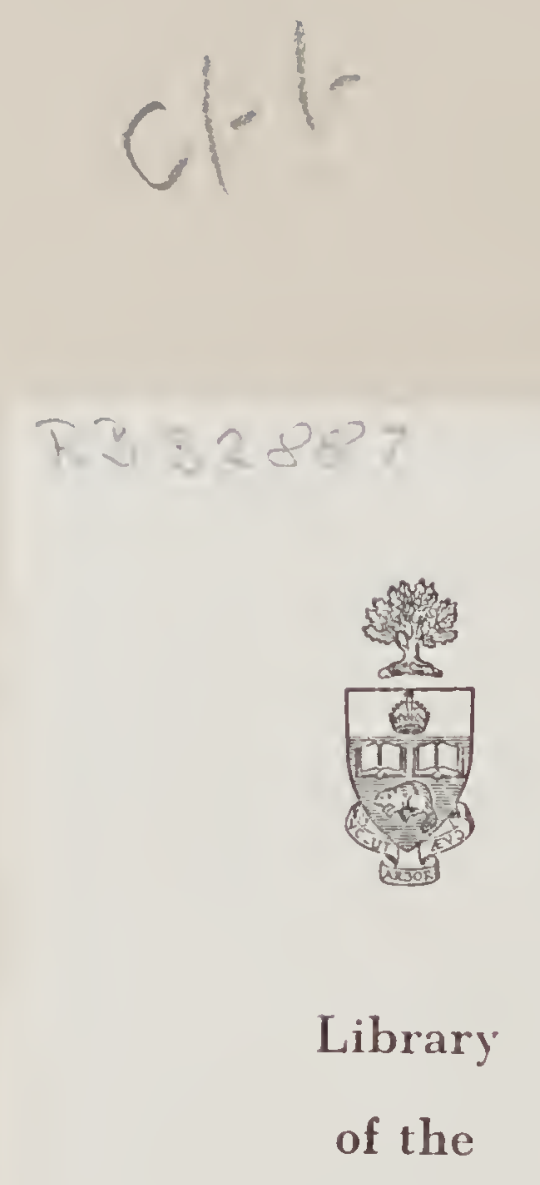

University of Toronto

THIS BOOR WAS SUPPLIRD BY ZOWARD BAKJR,

14 8 JOHN BRIGHT SIR

BI?MTCMA, שNGLA

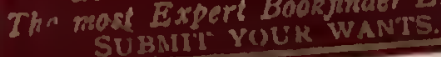



Digitized by the Internet Archive in 2020 with funding from University of Toronto 

BY THE SAME AUTHOR

STUDIES IN HETEROGENESIS

THE NATURE AND ORIGIN OF LIVING MATTER 


\section{THE}

\section{EVOLUTION OF LIFE}

$\mathrm{BY}$

H. CHARLTON BASTIAN, M.A., M.D., F.R.S.

EMERITUS PROFESSOR OF THE PRINCIPLES AND PRACTICE OF MEDICINE AND OF

CLINICAL MEDICINE IN UNIVERSITY COLLEGE, LONDON ;

CONSULTING PHYSICIAN TO UNIVERSITY COLLEGE HOSPITAL, AND TO THE,

NATIONAL HOSPITAI. FOR THE PARALYSED AND EPILEPTIC

IVITH DIAGRAMS AND MANY PHOTOMICROGRAPHS

METHUEN \& CO.

36 ESSEX STREET W.C.

LONDON 
First Published in 1907 


\section{PREFACE}

THE publication of my work "The Beginnings of Life" in I 872 gave rise to a good deal of criticism, and was followed by much controversy for a few years. The criticism concerned both sections of the work-that dealing with Heterogenesis, as well as the portion in which I endeavoured to establish the reality of Archebiosis, though there was controversy only in regard to the latter subject.

The absence of controversy about Heterogenesis was due to the fact that I made no reply whatever to any of the criticisms concerning this section of my book. It was a kind of subject which did not seem open to profitable discussion, especially with persons who declined to attempt to repeat the observations in question.

I was at the time, however, more sanguine of good resulting from a discussion concerning Archebiosis. Much controversy therefore followed with some very formidable opponents, and as a result I continued to do further work in reference to this question up to the year 1877 . During that and the previous year a very heated controversy was carried on with Professor Tyndall in this country and with $M$. Pasteur in France. Both were excessively dogmatic, and one at least showed little courtesy to his opponent ; so that, at last, a time came when, in my 
capacity as a comparatively young physician, I felt compelled to renounce these investigations for a time and to devote all my energies to professional work. The last article written by me on the subject of Archebiosis appeared in The Nineteent/ Century for February i $87 S$, in reply to one from Professor Tyndall.

Since that date I have published nothing on this subject except a single chapter last year in my work "The Nature and Origin of Living Matter." Still, though nothing had been published, I had, in the intervening years - from time to time, when opportunity permitted-done a good deal of work in reference to this question.

After having devoted most of my energies to professional and teaching work during the following twenty years, I resigned my teaching appointments at University College and Hospital at the beginning of 1898 , in order that all the time and energy that could be spared from private practice might then be devoted to a reinvestigation of the subject of Heterogenesis, concerning which I had been silent since ${ }_{1} S_{72}$.

The fact of my silence for the previous twenty years on both subjects may well have given colour to the notion, probably entertained by many, that I had been defeated and had given up the cause. Meanwhile the most extraordinary advances had been made by bacteriologists in many parts of the world, resulting in an increase of knowledge of vast importance for the science of medicine, and of a kind which doubtless seemed to many only compatible 
with views opposed to mine. There is, however, in reality no such irreconcilability between my views and modern bacteriological work. My views, in fact, serve merely to give a broader outlook to ætiological and sanitary problems.

This being the state of things, it may well be imagined that if I had not been imbued with the strongest feeling of the truth, in the main, of the views which I had previously published concerning Heterogenesis, I should never again have taken up this very unpopular subject and have worked hard at it for five years, till I had completed in I 903 my larger work entitled "Studies in Heterogenesis." During these years the subject was worked at, partly over new and partly over old ground, and this time with the aid of photomicrographs rather than with drawings, so as to avoid the bias more or less inseparable therefrom.

During the past year, in my leisure time, I have again been at work on the subject of Archebiosis, and have, I trust, done something at last which will carry conviction to very many as to the reality of the present de novo origin of living matter. The penultimate chapters of this book will show that in my new attempt to solve this old and fascinating problem, the experiments have in some respects been conducted in a new way, though by methods as notable for their simplicity as for their stringency in reference to all possible precautions. 



\title{
CONTENTS
}

INTRODUCTION . . . . . . . X . . .

\author{
PART I
}

THE MODERN ASPECT OF THE PROBLEMS

CHAP.

I. The Earth as one among a Multitude of Inhabited

Worlds . . . . . . . . . I

II. The Constitution of Matter . . . . . 4

III. Inorganic Evolution $\quad$. $\quad$. $\quad$. $\quad$. 10

IV. Organic Evolution as a Natural Sequence of Inorganic Evolution . . . . . . .

V. Some Modern Views and Present-day Misconceptions . $\quad 26$

\section{PART II}

THE CONDITIONS OF THE PROBLEM AND THE MODES OF EXPERIMENTATION

VI. Experimental Conditions as opposed to Natural Conditions : their Unfavourable Nature : . 35

VII. The Presence of Germs in Air and Water . . 39

VIII. The Limits of Vital Resistance to Heat: Early Observations . $\quad$ • . $\quad$. $\quad$. 43

IX. The Limits of Vital Resistance to Heat : later Observa-

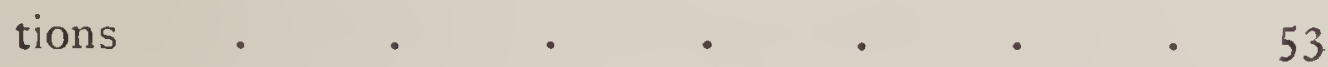

X. The Limits of Vital Resistance to Heat : Conclusion • 67

XI. Modes of Testing the Question whether certain Solutions can give Birth to Specks of Living Matter • 87 


\section{PART III}

THE EXPERIMENTAL EVIDENCE IN REFERENCE TO PASTEUR'S

\section{CONCLUSIONS}

XII. Was Pasteur right in saying that guarded Acid Fluids previously Heated to $100^{\circ} \mathrm{C}$. remain barren?

XIII. Was Pasteur right in his explanation of the Fact that guarded Neutral, or Slightly Alkaline, Infusions previously Heated to $100^{\circ} \mathrm{C}$. will often Ferment?

XIV. New Experiments in reference to the Fertility of Neutral Organic Solutions heated to $100^{\circ} \mathrm{C}$. .

XV. Discussion with M. Pasteur in reference to the Experiments recorded in the last Chapter, followed by the appointment of a Commission by the Academy of Sciences of Paris

XVI. Final Experiments on the Cause of the Fertility of Boiled Neutral, or Slightly Alkaline, Organic Solutions

XVII. Was Pasteur right in saying that Neutral or Slightly Alkaline Organic Fluids previously exposed to $110^{\circ} \mathrm{C}$. always remain barren?

\section{PART IV}

CONPLICATED METHODS AND CONFLICTING RESULTS

XVIII. Professor Tyndall's Experimental Evidence with Heated Organic Fluids

\section{PAR'T V}

NEW EXPERIMENTS WITH SUPER-HEATED SALINE SOLUTIONS

XIX. Objects and Methods in the New Experiments: Initial

Trials . . . . . . . 229

XX. Final I)ecisive Experiments . . . . . 259

\section{PART VI}

XXI. The Relation of my Work and Views to Modern Bacteriology • . . . . 295

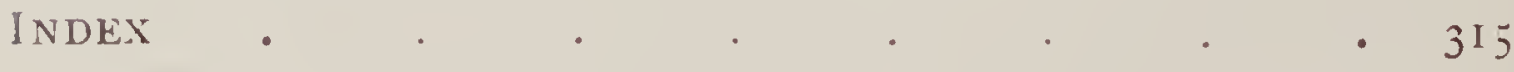




\section{LIST OF ILLUSTRATIONS}

\section{WOODCUTS}

FIG. I. Jeffries Wyman's Apparatus for Calcining the Air

2. Plugged Flask with charged Liquor Potasse Tube . 134

3. Burette Tube for Measuring Liquor Potassæ. . ${ }_{1} 8$

4. Mode of charging Liquor Potasse Tubes . . I 39

5. Lipped Measure . . . . . I4I

6. Sealed Retort containing charged Liquor Potassæ Tube . . . . . . I +3

7. Retort with Platinum Electrodes for Liberation of Oxygen . . . . . . . . 146

8. Pasteur's Double Tube. . . . . 175

9. Micro-organisms obtained from superheated Hay

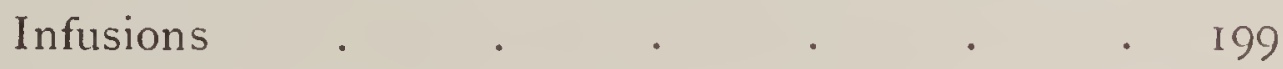

Io. Fungus Mycelium and Torulie from superheated Hay Infusions $\quad$. $\quad$. $\quad$. $\quad$. 203

II. Tyndall's Experimental Chamber . • . 222

12. Simple Experimental Tube, showing Deposit of Silica after the Solution has been heated . . . 233

\section{PLATES}

FACING PAGE

Plate I. Bacillus Spores, with Bacteria, Bacilli, Torulæ, and Fungus Spores, whose Thermal Deathpoint must be known (Figs. 1, 2, 3, 4) . 70

II. Organisms from Saline Solutions in Plugged Flasks, previously heated to $100^{\circ} \mathrm{C}$. for ten minutes (Figs. 5, 6, 7) . . .

111. Other Organisms from Saline Solutions in Plugged Flasks, previously heated to $100^{\circ} \mathrm{C}$. for ten minutes (Figs. 8,9 , 10) . 
FACING PAGE

Plate IV. Organisms taken from previously closed Tubes which had been heated to $100^{\circ} \mathrm{C}$. for ten minutes (Figs. I I, I 2, I3)

V. Other Organisms taken from previously closed Tubes which had been heated to $100^{\circ} \mathrm{C}$. for ten minutes (Figs. I 4, I 5, I6)

VI. Organisms taken from previously closed Tubes which had been heated to II $5^{\circ} \mathrm{C}$. for ten minutes (Figs. 17, I8, 19)

VII. Organisms taken from previously closed Tubes which had been heated to $120^{\circ} \mathrm{C}$. for ten minutes (Figs. 20, 21, 22)

"VIII. Organisms taken from previously closed Tubes which had been heated to $125^{\circ} \mathrm{C}$. for ten minutes (Figs. 23, 24, 25)

" IX. Organisms taken from previously closed Tubes which had been heated to $130^{\circ} \mathrm{C}$. for twenty minutes (Figs. 26, 27, 28)

X. Other Organisms taken from previously closed 'Tubes which had been heated to $130^{\circ} \mathrm{C}$. for twenty minutes (Figs. 29, 30, 31, 32) .

XI. Other Organisms taken from previously closed Tubes which had been heated to $130^{\circ} \mathrm{C}$. for twenty minutes (Figs. 33, 34, 35) upon the Saline Media thenselves (Figs. 36, $37,38)$

** For permission to reproduce the woodcuts shown in Figs. 2, 3, 4, 5 . $6,7,9$, and 10, my thanks are due to the Council of the Linnean Society; while for permission to reproduce Fig. II I have similarly to thank the Council of the Royal Society. 


\section{INTRODUCTION}

$\mathrm{A}^{\mathrm{S}}$ knowledge increases concerning any departA ment of science it almost always becomes necessary to give up some terms or modes of expression that have been long in use; either because they convey notions absolutely irreconcilable with the later development of knowledge, or because they are too vague and general. Hence it is that the phrase "spontaneous generation" should be rejected at the present day. The phenomena hitherto referred to under this name would be no more "spontaneous" than are any others which take place in accordance with natural laws. The phrase is, moreover, quite inadequate, since under it, if retained, we should have to include two sets of phenomena which, in the present day, ought to be carefully discriminated from one another.

Many who have written on the subject of "spontaneous generation" have, however, failed to appreciate the full extent of the difference that exists between the origin of living things from not-living materials (Archebiosis), and their production, in whatever fashion-whether by modes that are familiar or by others which are unfamiliar-from the substance of pre-existing living things. This difference, which is so little dwelt upon by some, assumes in the minds of others an overwhelming 
importance. They might be open to conviction as to the possibility of living things arising by previously unknown methods from the matter of pre-existing living things (Heterogenesis); while they would regard the origin of living things from not-living materials to be altogether impossible.

It may be easily understood that the general views entertained concerning Life--its nature, or the meaning to be attached to it as a term-exercise no small influence in producing a variation in the point of view of different writers in regard to Archebiosis. Thus, statements which would appear to many to be consistent only with a belief in Archebiosis are, when taken in conjunction with the general views of the writers, often found not to warrant any such conclusion.

This may be illustrated by reference to the views of Burdach, the physiologist, from whom the term "Heterogenesis" has been derived. In the first volume of his "Physiologia," published in I $\$ 26$, Burdach introduced the words Homogenia and Heterogenia, as names for the two principal class distinctions in the mode of origin of living things. Homogenia was the class name applied to the processes by which individuals result from preexisting living things similar to themselves in organisation; while Heterogenia was the class name for processes by which living things arise from the matter of pre-existing organisms belonging to a totally different species - the production, that is, of alien forms of life from the actual substance of organisms or their germs. 
But Burdach gave to the term Heterogenesis a wider signification still : he made it include the process of Archebiosis also, as may be seen when he says ${ }^{1}$ :"Nul doute, que notre planète ne soit arrivée par degrés à son état actuel, qu'à une époque très reculée elle n'ait-été inhabitable pour les êtres organisés, et que tous ces êtres ne soient formés peu à peu sans parens, consequemment par la voie de l'hétérogenie."

Although this passage shows that Burdach believed in the possibility of the origin of living things from what are called not-living materials, nevertheless he did not believe that in such a case there would be a creation of the something altogether new, which we term "Life." This divergence arises from the nature of his theoretical views. The whole universe was to him the organism of organisms, endowed with Life. Thus, elsewhere he says":- "Mais si l'univers est l'organisme absolu, chacune de ses parties doit être un tout organique ... Il y a plus encore: la force du tout doit être inhérente à chaque chose particulière, et effectivement nous rencontrons des traces de vie dans toute existence quelconque."

Very similar vitalistic or pantheistic doctrines were also entertained by Buffon, Needham, and Pouchet. Each of them believed that pre-existing "vital force" of some kind-pre-existing Life therefore-was necessary, and that without the agency of

1 In the second edition of his work, as translated by Jourdan,

"Traité de Physiologie," I837, c. i. p. 8.

2 Loc. cit., t. iv. p. 149. 
this no living thing can come into being. They also were heterogenists in the wide sense given to this term by Burdach, and their theoretical views would not have allowed them to believe in what we have been speaking of as Archebiosis.

The term Heterogenesis is, however, always used by me in the more limited sense originally given to it by Burdach in his definition, as the class name for processes by which living things arise from the matter of pre-existing organisms belonging to a totally different species. When such a process occurs, it is a matter of altogether secondary importance whether the individualisation of a portion of the matter of an organism (with power of independent development) takes place during the life of such organism or after its death-since the death of any of the higher organisms does not at once entail the death of the matter entering into its composition. Its constituent parts continue to live for a time, and gradually, at different intervals, they lapse into the condition of mere dead matter.

When this stage comes, when the living substance is dead, we still have to do with organic matter composed of highly complex molecules - though now it is soluble in water. But when such matter has given up its semi-solid form and has undergone solution, it is in no sense, in accordance with commonly received views, to be regarded as living; so that if, in a solution thus formed, the evidence were to point to the de novo origin therein of living units, we might quite legitimately speak of it as a process of Archebiosis. Although the matter in solution may 
have once entered into the constitution of living things, it is now, in the ordinary acceptation of the term, dead matter - just as much as the matter contained in an ammoniacal saline solution is dead matter. It is true that the molecules in the organic solution are much more complex, and that, on this account, it might reasonably enough be considered to be a more easy process to bring about a new lifeorigination therein than in a more simple saline solution. The principal lesson that we may learn from such a fact would be, that the process of Archebiosis, which so many believe to have undoubtedly occurred in the past (when hitherto there had been no living thing on the face of the earth) ought to be much more easy of occurrence in the present day when organic matter in solution is so widespread over its surface.

The foregoing views, and the radical distinction between the processes of Archebiosis and of Heterogenesis, may perhaps be more fully brought home to the reader by the following table reproduced from my work, "The Beginnings of Life."

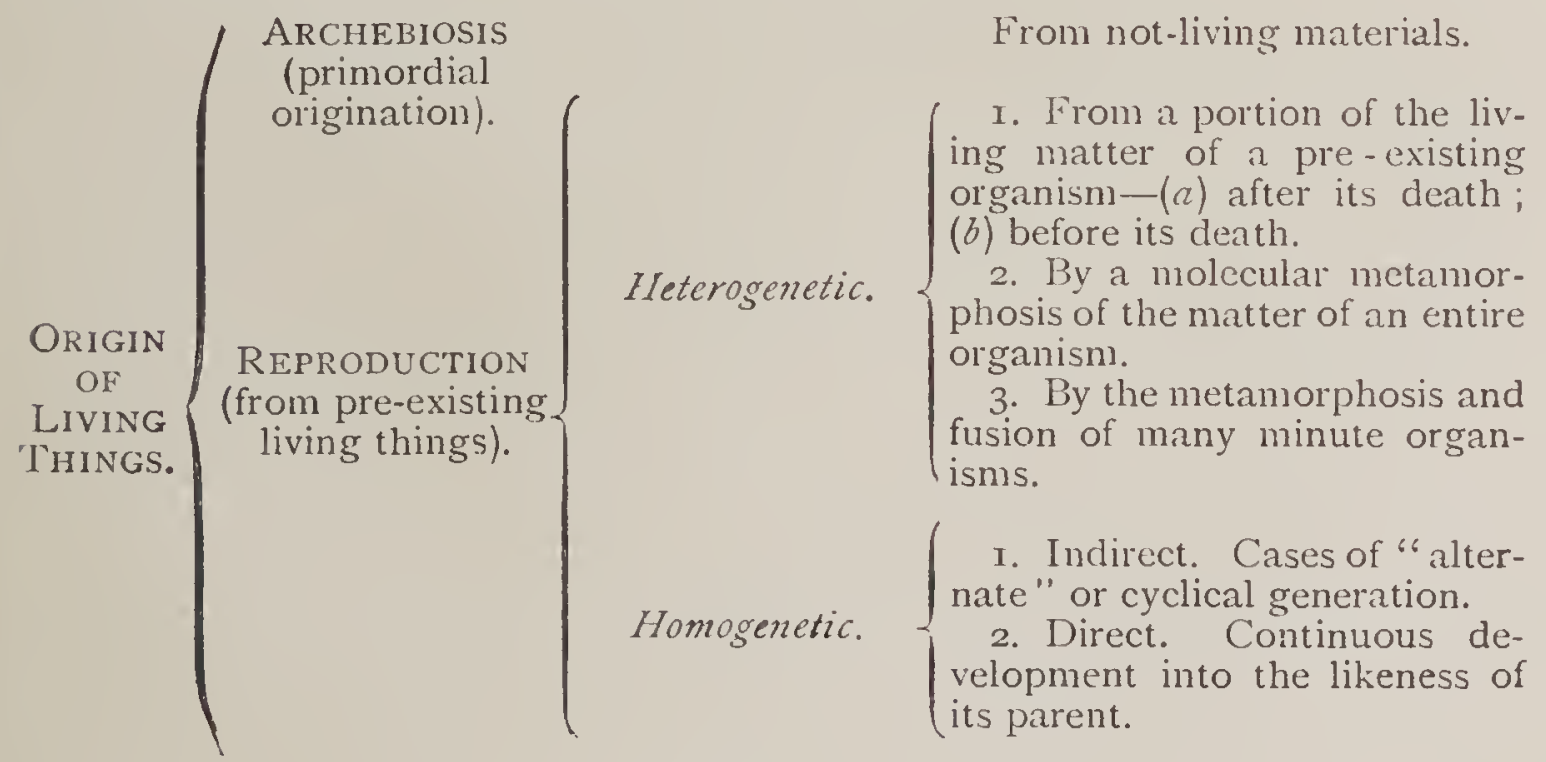


In the present volume I shall not deal at all with Heterogenesis. Five years of independent work have been recently devoted by me to this subject, and the results were published in my "Studies in Heterogenesis" (I901-1903); while portions of these results, together with my views on the subject of living matter generally, have been embodied in a more popular form in my recently published book on "The Nature and Origin of Living Matter."

Here we shall limit ourselves to the problems connected with Archebiosis - that is, to the question of the actual origin of life or the genesis of living matter. I shall, moreover, limit myself to a consideration of the evidence bearing upon this question which has accumulated since 1872 , when my work, "The Beginnings of Life," was published. An account of the earlier investigations on this subject, from the time of Spallanzani and Needham onwards, will there be found, which it would be comparatively useless to attempt to repeat in a much condensed form. It seems better to devote all available space to a fuller consideration of the more modern aspect of the problem and more recent experimental work-and, moreover, to take a broad outlook upon the question generally, in the light afforded by modern investigations on the constitution of matter, on the "mystery of radium," and on "inorganic evolution." 


\title{
THE EVOLUTION OF LIFE
}

\author{
PART I \\ THE MODERN ASPECT OF THE QUESTION \\ CHAPTER I
}

THE EARTH AS ONE AMONG A MULTITUdE OF INHABITE,D WORLDS

STRONOMERS tell us that among the billions
of suns which people space, thousands have orbs circling round them which are, in all probability, at this present time, the abode of living creatures of some kind.

For, as Professor Simon Newcomb says, " "In a number of bodies so vast we should expect every variety of conditions as regards temperature and surroundings," so that if we suppose "the special conditions which prevail on our planet are necessary to the highest forms of life, we still have reason to believe that these same conditions prevail on thousands of other worlds."

That this is no mere individual and isolated opinion can easily be shown. No man was more cautious and exact in the expression of his opinions

${ }^{1}$ Harper's Magazine, August 1905.

A 
than the late Sir George Stokes, a former president of the Royal Society, yet in his "Lectures on Light" he says, in reference to the stars: "We can hardly suppose that life is confined to one particular planet circulating round one particular sun out of this vast multitude . . . We can hardly avoid surmising that these distant suns may, like our own sun, be accompanied by planets circulating around them, and that these planets again, or such of them as may be habitable, are like our own earth, tenanted by living beings, it may be by rational beings of some kind" (loc. cit. pp. 243, 199).

And although life has undoubtedly existed on the surface of our earth for an enormously long period, yet, as R. A. Procter pointed out," "the whole duration of life must be regarded but as a wave on the vast ocean of time, while the duration of the life of creatures capable of reasoning upon the wonders which surround them is but as a ripple upon the surface of such a wave." Thus, the infinity of habitable worlds implies also the existence of an infinity of worlds not as yet habitable, or which have long since passed their period of habitability.

If the number of suns with their attendant planets is so vast as to transcend all human powers of imagination, still more hopeless would be the attempt to form any conception of the infinite realms of space through which these orbs are distributed. What is supposed to be the nearest star, situated in the constellation Centaur, is so 1 "Our Place among Infinities," p. 6r. 
distant that, as computed by Prof. Bickerton,1 " it would take the swiftest express train forty millions of years to get to the nearest star"-while even light, with its enormous velocity, would take over four years to travel from it to us, and probably thousands of years to reach us from one of the most distant suns.

Yet all through this stupendous universe, as Bickerton says, "the spectroscope reveals identity of matter and community of motion."

Not only are the same elements that exist on this earth found in the stars, but, as Sir Norman Lockyer tells us ${ }^{2}$ - and the statement, as we shall see, is one of the greatest importance-they are found in gradually increasing numbers, "as we come down from the hottest stars to the cooler ones." The number of spectral lines, as he says, gradually increases, "and with the number of lines the number of chemical elements." The full significance of these statements will be revealed in the next two chapters.

1 "The Romance of the Heavens," I901, p. I62.

2 "Inorganic Evolution as studied by Spectrum Analysis," I900, p. 159 . 


\section{CHAPTER II}

\section{THE CONSTITUTION OF MATTER}

$\prod \begin{gathered}\mathrm{HE} \text { atomistic conceptions of Leucippus and } \\ \text { Democritus have acquired new interest owing }\end{gathered}$ to the results of modern experimental work concerning the constitution of matter. All substances, they taught, were primarily formed of atoms too small to be apprehended by the senses: indivisible and unchangeable, though differing greatly in shape and size. These atoms were supposed to be constantly in motion and separated "by a void." The qualities of different bodies were thought to be due in part to the kind of atoms existing, and in part to their arrangement in the bodies in question. Aristotle put it thus: "Democritus and Leucippus say that all things are composed of indivisible bodies, and that they are infinite both in number and in their forms, and that the differences between things are due to the elements of which they are composed and to the position and arrangement of these elements."

Such views were, of course, mere speculations having no basis either in observation or in experiment. But, like the modern "Atomic Theory," promulgated by Dalton in the early years of the last century, these speculations rejected the notion of 
one primordial kind of matter, to which other philosophers in intervening years had leaned. Again, the atoms of the early philosophers were indefinite in point of number, while those of Dalton were strictly limited to the number of the different chemical elements known to exist in his time. Each atom was supposed by him to have a weight characteristic of, and peculiar to, the element to which it belonged; and all were believed to be indestructible. Dalton said, " "we might as well attempt to introduce a new planet into the solar system, or to annihilate one already in existence, as to create or destroy a particle of hydrogen "-a view which, as we shall see, no longer holds good for all of the so-called elements.

But Dalton's notion of an uncertain number "of elementary principles which can never be metamorphosed one into another by any power we can control," was, in the latter third of the last century, gradually being superseded by the view, as Sir William Crookes" put it, "that our so-called elements or simple bodies are in reality compound molecules," made up by varying combinations of some extremely minute primordial element, which, confessedly hypothetical, he spoke of as protyle.

Crookes, however, demonstrated the actual existence of particles very many times more minute than the chemical atoms, as constituents of the so-called "cathode rays" - and resulting from the splitting up of gases in vacuum tubes by electricity.

1 "New System of Chemical Philosophy," I 808.

2 "Genesis of the Elements," Chomical Neres, 55, 1887, p. $\&_{3}$. 
Later, the researches of Professor J. J. Thomson ${ }^{1}$ and his school have shown that in the conduction of electricity through highly rarefied gases the particles shot out from the cathode (or negative pole), constituting the above-mentioned cathode rays, always carry an electrical charge identical with that conveyed by a single atom of hydrogen, though each particle in these cathode rays associated with this constant minimum charge has only about $\frac{1}{1000}$ of the bulk of the hydrogen atom; and, more important still, that the particle is always of the same kind and size whatever the substance may be from which the cathode ray emanates. Such particles, all identical in mass and in the charge which they carry, are by Professor Thomson named "corpuscles," though they are more commonly spoken of as "electrons"a name previously suggested by Dr Johnstone Stoney, and indicative of the view now held by many that these elementary corpuscles are in reality electrical units.

These "corpuscles " or "electrons" are indeed now supposed to be the elementary units out of which the atoms of the various chemical elements are compounded-their number in each atom being very great, and increasing with each increase in the atomic weights of the several elements.

Thus, while an atom of hydrogen whose atomic weight is very low (I) has been supposed to contain I000 of these corpuscles or negative electrons, an atom of mercury, with a very high atomic weight (200), has been supposed to contain I00,000 of such 1 "Conduction of Electricity through Gases," 1903. 
elementary units. What seems, however, more astounding still is the fact, as stated by Sir Oliver Lodge, that even with these vast numbers in a single atom the corpuscles do not "fill all the space, and if the distance between them were calculated they seemed to be about as far apart, in proportion to their size, as the planets in the solar system." It is, in fact, now supposed by many authorities that there is "in the case of the atom, nothing but a group of positive electrons, forming a body like our sun, round which their negative partners revolve at distances and in orbits corresponding not imperfectly to those of the planets ... and the difference of chemical and physical behaviour displayed, for instance, by an atom of hydrogen and another of iron is accounted for by supposing the planets of one to be either more numerous, or to have different orbits from those of others." 1

This last is an all-important statement, but it is needless for us here to attempt to follow the actual investigations further. What they have led to is this: that the corpuscle or electron is the infinitesimally minute primordial substance, the compounding of which in vast and gradually increasing numbers gives rise to the several collections of properties met with in the atoms of the different so-called elements. In what precise way these combinations are brought about we know not; though in the next chapter some of the conditions will be made known that appear to have favoured the process, and which permitted the locking up, 
within the atoms, of the vast stores of energy which they are now known to contain.

Radium, thorium and uranium are the three chemical elements whose atomic weight is the highest: and how great is the difference, in this respect, between the several chemical elements may be gathered from the fact that the atomic weight of hydrogen being I, that of uranium is 239. Think what this means in the way of atomic complexity for uranium, when even the atom of hydrogen is supposed to contain no less than Iooo corpuscles. How vastly complex must be the atoms of these higher terms of the series, such as radium, thorium, and uranium! And how vast, too, must be the amount of intratomic energy locked up within such larger atoms may be dimly understood when we are told that Professor Thomson "as the result of his calculations, concludes that a grain of hydrogen has within it energy sufficient to lift a million tons through a height considerably exceeding one hundred yards; and that since the amount of energy is proportional to the number of corpuscles comprising the atom of the element, the energy of the other elements such as sulphur, iron, or lead must enormously exceed this amount." 1

From what has been said it may be gathered how impossible it must be to form any adequate conception of the complexity of matter, when we consider not only this marvellous constitution of the very atoms of the elements, but the fact, as we know, that these atoms ever tend to combine with one

${ }^{1}$ I uncan, "The New Knowledge," 1905, p. 176. 
another to form more and more complex moleculessuch as are to be met with in bases and acids, in salts and double salts, in alkaloids and organic compounds - some of the latter of which may be composed of hundreds of the marvellous atoms whose constitution we have been brielly considering.

Can anything be said as to the mode of origin of these chemical elements, or as to some of the conditions that seem to favour their origin? Some hints on this subject must be reserved for the next chapter. 


\section{CHAPTER III}

\section{INORGANIC EVOLUTION}

IF the atoms of the various chemical elements are in reality such complex bodies as the researches of physicists indicate, and especially if they are compounded of vastly different numbers of infinitesimally small corpuscles all of one size, merely varying in mode of arrangement and in their numbers and relative motions, it seems natural to ask whether any evidence can be obtained bearing (a) upon their genesis, or (b) upon their decomposition or disintegration.

Modern research has furnished us with something in both directions. The former kind of evidence has been derived from the stars, as studied by spectrum analysis; while for the latter knowledge we are indebted to quite recent researches-that is, to some of the astounding revelations concerning radium.

The spectroscope has been assiduously used during the last forty years by Sir Norman Lockyer in studying the constitution of the sun and the stars. And his results, together with those of other workers in the same field, have led to the gradual building up of the doctrine of "Inorganic Evolution." Without the aid of the spectroscope no such know- 
ledge could ever have been obtained. Concerning this instrument and what it is capable of revealing we find Professor Duncan in his very interesting work, "The New Knowledge," saying, "That the spectroscope will detect the millionth of a milligram of matter, and on that account has discovered new elements, commands our admiration; but when we find, in addition, that it will detect the nature of forms of matter billions of miles away and, moreover, that it will measure the velocities with which these forms of matter are moving with an absurdly small per cent. of possible error, we can easily acquiesce in the statement that it is the greatest instrument ever devised by the brain and hand of man."

Though spectrum analysis is a very complicated subject, upon which an enormous amount of work has been done, not many details need be referred to here. Points of major importance are that under certain conditions the different elements when volatilised by heat, and viewed through the spectroscope, give rise to different discontinuous or line spectra, and that no two elements yield precisely the same spectra.

The occurrence of these distinctive line spectra is dependent, as Norman Lockyer says in his work on "Inorganic Evolution," upon the law that "gases and vapours, when relatively cool, absorb those rays which they themselves emit when incandescent," and upon the existence of the particular conditions above referred to that lead to the production of the line spectra. For the heavenly bodies these condi- 
tions are to be found in the fact that "in the sun, and in most of the stars, we have light from a more highly heated centre passing through an envelope of cooler vapours, and on this account absorption phenomena are produced," thereby giving rise to the various groups of lines seen in the spectra. By the use of the spectroscope, therefore, and after prolonged labours devoted to the identification of the various sets of lines characterising the spectra of the different elements, we have gradually been taught that very many of the same elements which exist on this earth are to be found, in various combinations, entering into the composition of the sun and of the stars, and, what is all-important, in gradually increasing numbers as stars of lower temperature are brought under observation.

In the early days of spectroscopic investigation it was supposed that an element could yield only one particular line spectrum. The first blow was given to this conception in 1865 by Plucker and Hittorf, who announced that "there is a certain number of elementary substances which, when differently treated, furnish two kinds of spectra of quite a different character, not having any line or band in common." Much work has since been done in this direction by Sir Norman Lockyer, and his results with those of other workers have gone far to establish upon a firm basis the doctrine of Inorganic Evolution with which we are now concerned.

It soon became recognised that the spectrum of an element depended, within certain limits, upon the temperature to which the substance was exposed; 
and that the same element might, as many of them did, give totally different spectra when subjected to the following markedly increasing degrees of heat :-

(a) The temperature of a flame.

(b) The temperature of the electric arc.

(c) The temperature due to an electric spark of very high potential.

When examined in the laboratory at these different temperatures the spectra of iron, of magnesium, of calcium and other elements are found to vary in ways which seem incapable of explanation except on the assumption that the changes when passing from $(a)$ to $(b)$ or $(c)$ are due to dissociation of the elements under the influence of the high temperatures to which they are subjectedthat is, that the simplification of the spectra thus produced is due to the breaking up of their atoms into simpler groupings of corpuscles or electrons.

The significance of these variations has been vastly increased owing to the fact that precisely the same kind of changes which are seen in the laboratory are also recognisable in the spectra of the elements as they exist in the chromosphere of the sun, and in the light emanating from many stars. Concerning the latter bodies Kennedy Duncan says (loc. cit. p. 204): "The spectra of the stars afford in many cases the same simplified spectra observed in the sun of iron, magnesium, and calcium. In addition, simplified spectra of other metals are discovered, such as titanium, copper, manganese, nickel, chromium, vanadium, and strontium. To these supposedly broken-down metals the prefix proto is 
applied. By proto-iron, proto-copper, and protonickel, for example, is meant the constituents of iron, copper, and nickel as they exist in the sun or hottest stars. A very important proto-element is protohydrogen, discovered some time ago by Professor Pickering of Harvard University, in the star named zeta-Puppis in the constellation Argo. The spectral lines representing this substance Pickering at first supposed to signify a new element; but he was able to show, later, that they belonged to a new series of hydrogen lines constituting a form of hydrogen unknown on earth. Subsequently, this same proto-hydrogen was discovered in the stars 29 Canis Major and gamma-Argus. It is interesting to note that this broken down or proto-hydrogen is confined to the very hottest stars known. . . . If we accept the dissociation hypothesis, these curious proto-spectra are naturally, simply, and sufficiently explained. If we reject it, we shall look in vain for any other present-day explanation which will co-ordinate and harmonise the observed results."

Looked at from the point of view of the elements entering into their composition, Sir Norman Lockyer divides the stars into three main groups, namely: ( I) gaseous stars, (2) metallic stars, and (3) carbon stars - each representing different grades of temperature. In the former, or hottest stars, there are found, almost exclusively, the gases hydrogen, helium, and the gas asterium, which is as yet unknown on earth. In the stars of medium temperature, these gases become replaced by metals in the dissociated state in which they exist in an 
electric spark of extremely high potential. While in the third group, or stars of lowest temperature, the gases disappear almost entirely, and the metals exist in the state produced by the electric arc, that is, with more complicated spectra.

This is the kind of evidence afforded by a study of the stars. It is surely most impressive. We find the hottest stars containing the fewest elements, and these gradually increasing in the cooler stars. Nay, more, the elements which appear first are the lightest (that is, have the lowest atomic weights), and as a rule they appear in the order of their atomic weights. Again, the metallic elements always first appear in their dissociated or simplified state, and later in their normal form. Thus we have laboratory evidence of the dissociating effect of great heat upon the elements; and we have stellar evidence strongly pointing to the fact that as the stars cool, and the disintegrating effects of heat diminish, different chemical elements come into being, by reason of more and more complex combinations of some primordial element - the hypothetical "protyle" of Crookes, or what he subsequently made known to us as constituents of the cathode rays, the "corpuscles" or "electrons" of modern science.

\section{The Disintegration of Atoms}

The above-mentioned evidence supplied by the spectroscope for the occurrence of "dissociation," that is, disintegration of the atoms of elements under the disruptive influence of heat, has been rendered 
all the more real and forcible by modern investigations concerning radium-in which a spontaneous disintegration seems to be constantly occurring in some of its highly complex atoms. Like changes are known to occur in thorium, uranium, and actinium, and will probably be met with in other elements.

The astounding discovery made in I90I by Professor and Madame Curie that radium maintained itself at a temperature nearly $3^{\circ} \mathrm{F}$. above that of the surrounding atmosphere, was first explained by Professor Rutherford and F. Soddy as due to a spontaneous disintegration of the radium atom, and the bombarding of its substance by the corpuscles and alpha rays thus liberated-acting as minute projectiles and moving with inconceivable rapidity. They showed also that among the products of disintegration there were, in an "emanation," atoms of a density comparable with those of hydrogen and helium.

Subsequently Sir William Ramsay, in conjunction with F. Soddy, collected this gas, or emanation, evolved from salts of radium, and, writing on this subject, the former investigator says " : "We showed that this gas, presumably of high density, disintegrates in its turn, and that perhaps 7 per cent. of it changes into helium. What becomes of the remaining 93 per cent. is as yet undecided; still some hint may be gained from the fact that a constant ratio exists between the amount of helium obtainable from a mineral and the weight of lead which it contains. It may be that lead forms the

1 The Athencum, March 10, 1906, p. 301. 
ultimate product, or, at least, one of the ultimate products of the disintegration of the atom of emanation. Another radio-active element, actinium, has been shown by its discoverer Debierne also to yield helium by the disintegration of the emanation, or gas, which it continuously evolves."

Here, then, we have actual proof of transmutationa realisation to some extent of the dreams of the alchemists - in the resolution of radium and actinium into their emanation, and of these emanations into helium : a gas originally discovered in the chromosphere of the sun, and, after twenty-eight years, found on earth as a product extracted from cleveite, an ore of uranium in which it is always to be found. In addition, helium is now known to exist in many of the stars: namely in Capella, Arcturus, Pollux, Sirius, and Vega.

The disruptive changes occurring in the atoms of radium and actinium are explosions, and, as Sir William Ramsay says, "in principle an analogy may be drawn between the disruption of the molecules of an explosive compound and the disintegration of an atom into corpuscles." All ordinary explosions are known to be associated with a rise in temperature, and as such a process seems to be continuously going on in some of the atoms of radium, we have in this fact alone a partial explanation of the spontaneous production of heat in radium, which caused so much surprise among chemists and physicists when it was first made known.

How far similar disruptive changes are taking 
place in the atoms of other elements will doubtless soon be made known. They are conjectured by Gustave le Bon ${ }^{1}$ to be occurring in many others, and he regards "corpuscles" or "electrons" as intermediate stages between what we know as matter and the ether, from which, in ways altogether unknown, the different so-called elements have been, and are still supposed to be constantly forming during the process of evolution of new worlds-that is, in stars which are gradually cooling.

1 "L'Evolution de la Matière," Paris, 1905. I.e Bon supports the view that electrons are "centres of vortical strain in the ether." 


\section{CHAPTER IV}

ORGANIC EVOLUTION AS A NATURAL SEQUENCE OF INORGANIC EVOLUTION

FROM what has been said it seems perfectly clear that the scientific discoveries of recent years have been of a kind strongly tending to establish the truth of the doctrine of inorganic evolution, by which, as Professor Duncan says (loc. cit. p. 206), "we mean that the eighty odd elements of matter as we know them on earth to-day were not specially created, but that, like the plants and animals, they have been truly evolved from simpler and still simpler types, back to some really simple element from which they have all evolved through infinite æons gone by." We cannot, indeed, refuse our assent to his view when he adds: "Taking it altogether, the evidence for an inorganic evolution of the elements seems every whit as conclusive as the evidence for an organic evolution. ... The geologist from an examination of the earth's strata from lowermost to highest finds an ever-increasing complexity in the organic remains which the rocks contain. The astronomer from an examination of the stars from hottest to coldest finds an ever-increasing complexity in the so-called elements which they contain. ... They both 
deduce an evolution of simpler forms to more complex, and their deductions are equally valid; we must accept the inorganic evolution. Organic evolution is measured by millions of years; inorganic evolution is measured probably in billions" (p. 2 I 2 ).

The next question that presents itself is as to the relation between these two processes of evolution, and especially as to the starting-point of the latter process - that of organic evolution.

Let us look at the question first from a broad point of view. We have seen that there is a community of nature between the earth and all the infinite myriads of stars; that the same chemical elements in varying number are to be found existing in them as are to be met with on this earth; and that such elements are, as it would seem, gradually being evolved in the stars, under the influence of all-pervading natural laws, as the process of cooling goes on. We have, further, every reason for believing that space is peopled by myriads of planets which are to us wholly invisible-by bodies, that is, which, like our earth, have been thrown off from other suns.

We know that in the far-remote past when the surface of our earth cooled down, when oceans and an atmosphere had come into existence, chemical changes must have progressed, and that at last a new kind of synthesis must have taken place - a synthesis resulting in the formation of what we call "living matter." Men of science no longer doubt that a natural birth of living matter must have occurred in 
the past when the surface of the earth had become sufficiently cool; and such a process could only be regarded as a continuation and sequence of that related process of evolution which had previously been leading to the genesis of the various chemical elements and of those simple combinations among them, by which oxides, acids and other compounds are produced.

All the combinations in question-whether of electrons, of atoms, or of molecules, and whether simple or complex-being the work of natural influences and natural affinities, it is only reasonable to suppose that the particular combinations giving rise to living matter, when the right time came, would have occurred in multitudinous regions over the earth's surface, and would have recurred again and again as long as the conditions remained favourable.

How these particular combinations were led up to -what were the actual steps of the process-no one can say, but that they must have occurred no person possessing a fair amount of chemical and biological knowledge now doubts. And if this beginning of organic evolution is but a continuation and natural appendix to what had gone before; if, as astronomers tell us, there must be thousands of suns having orbs circling round them like our earth, and among these perhaps thousands in which the special conditions existing are very similar to those now existing, or that have existed, on our planet, we are fully entitled to believe that just as the simple chemical elements are ever coming into being in untold myriads of 
suns, so, throughout the universe, there may be thousands or even millions of planets in which the highest kind of synthesis, represented by living matter, has been or is taking place.

Such a process when once it was started on this earth-perhaps some hundreds of millions of years ago-has been followed by the evolution of all the increasingly complex forms of life that have lived in past ages (some comparatively few examples of which have happily been preserved for us in successive geological strata), together with all the innumerable forms that still people the earth and dwell in its oceans.

Since the great revolution in scientific beliefs brought about by the epoch-making work of Darwin, it is now generally admitted by men of science that all the various forms of life have arisen one from another by processes of evolution-that they have appeared, that is, as successive natural results of the interaction between the primordial forms of living matter and the sum-total of the influences, animate and inanimate, acting thereupon. Thus these multitudinous forms of vegetal and of animal life are deemed to have come into being as final results of those same physico-chemical processes which we have seen to be in operation throughout the universe. Starting from the all-pervading ether - whose origin and nature are alike inscrutablemodern-day physicists would have us regard it as the source in and from which the primordial units of matter (the electrons) arise. How these aggregate (perhaps first in their tens and hundreds and then in 
their thousands) to form the various chemical elements, we are just as ignorant as we are concerning the formation of those ultimately complex material combinations that lead on to the production of living matter.

Yet the spectroscope compels us to believe that the processes which lead to the genesis of so-called chemical elements are continually occurring throughout the universe; while astronomical and geological evidence combined assure us that a genesis of living matter must have occurred at some time in the fardistant past, when the temperature of the earth's surface had sunk below the boiling point of water.

Thus, in the formation of electrons; in the formation of the eighty or more different chemical atoms ; in the formation of more and more complex chemical compounds, terminating in those met with in living matter; and in the production of all the forms of life that have since appeared, we have a continuous series of evolutional results, each more or less incapable of explanation, yet all dependent upon natural processes acting, as it would appear equally, throughout all space and all time.

This seems to be the natural and logical point of view from which to approach the all-important question, whether the genesis of living matter is or is not still taking place on our earth at the present day-a process which we shall henceforth speak of under the name of "Archebiosis."

The question that has been so much discussed of late years is the strange one, whether such a process 
occurred only once or more in some remote geological ages, as many are inclined to think; or whether it is a process that has been going on ever since it first began, all through the ages, and is one still continuing to occur at the present day.

When it was said, a few pages back, that "no person possessing a fair amount of chemical and biological knowledge" now doubted that a natural origin of living matter had in the past occurred on this earth, it must not be forgotten that some years since (in $\mathrm{I} 87 \mathrm{I}$ ) Lord Kelvin, adopting a view previously expressed by another celebrated physicist (Helmholtz), threw doubt upon the subject by suggesting that life might have come to us on "the moss-grown fragments of another world." This evasive and very improbable view seems now altogether beside the question, and as was pointed out by Sir George Stokes ${ }^{1}$ would, of course, "leave untouched the problem of the origin of life." This eminent physicist added, "I need not dwell, however, on the very formidable difficulties which stand in the way of any such hypothesis, for example, the intense superficial incandescence produced in a meteorite by its rapid passage through the air, for I do not conceive that the hypothesis was ever meant to be adopted."

Some astronomers, indeed, so far from doubting that Archebiosis originally occurred on the earth, go so far as to think that life of some sort is to be found even on other planets belonging to our own solar system. Thus, Dr H. H. Turner, Professor of

1 "Lectures on Light," I S92, p. 33 I. 
Astronomy in the University of Oxford, lecturing at the Royal Institution last December, is reported to have said, "All the stars that we saw [that is, all visible stars], with a few insignificant exceptions, had a temperature somewhat like that of the sun, and therefore they too must be destitute of life. But there might be comparatively cool bodies circling round the stars in the same way as the planets round the sun, and to these the objection of high temperature might not apply. To the question are the planets inhabited, his answer would be that, in the absence of evidence he did not know, but that he felt pretty sure they were, because they were so like the earth in many particulars, that they might be supposed to be like it in this one." 


\section{CHAPTER V \\ SOME MODERN VIEWS AND PRESENT-DAY \\ MISCONCEPTIONS}

IN accordance with the facts set forth in the 1 previous chapters, there would be no more reason for postulating a miraculous interference or exercise of Creative Power to account for the evolution of living matter in any suitable portion of the Universe (whether on this Earth or elsewhere), than to explain the appearance of any other kind of matter-the magnetic oxide of iron, or gold, or radium, for instance.

But to suppose the formation of living matter to have occurred once only, or to have been limited to the time of its first appearance on this earth, instead of imagining that, like other physico-chemical changes, its production is, and has been, an everrecurring process, would be tantamount to regarding it as a quasi-miraculous process - a something not occurring under the influence of natural laws. The language used, however, and the attitude taken by some of the leading exponents of the doctrine of Evolution in this country has unquestionably tended to foster some such view.

Thus speaking of the probable commencement of Life upon our globe, Darwin said ${ }^{1}$ : "I believe that

1 "Origin of Species," 6th ed., I872, pp. 424 and 429. 
animals have descended from at most four or five progenitors, and plants from an equal or lesser number. Analogy would lead me one step further, namely, to the belief that all animals and plants have descended from some one prototype. . . . There is grandeur in this view of life, with its several powers, having been originally breathed by the Creator into a few forms or into one; and that whilst this planet has gone cycling on according to the fixed law of gravity, from so simple a beginning, endless forms, most beautiful and most wonderful, have been and are being evolved."

Herbert Spencer, of course, made no sort of appeal to a Creative Hypothesis. He distinctly taught that living matter must have been the gradual product or outcome of antecedent material combinations. "Construed in terms of evolution," he said, "every kind of being is conceived as a product of modifications wrought by insensible gradations on a pre-existing kind of being, and this holds fully of the supposed 'commencements of organic life,' as of all subsequent developments of organic life."

But, on the question whether the process of Archebiosis is likely to have occurred once only, as Darwin seemed to hint, or in multitudinous centres scattered over the earth's surface, Herbert Spencer made no definite statement. The latter belief would, however, be entirely in accordance with his general doctrine; and we seem all the more entitled to infer that he inclined to the notion of a multiple occurrence of Archebiosis, both in space and in 
time, since he did not reject the possibility of its occurrence in our own day. Granting "that the formation of organic matter and the evolution of life in its lowest forms may go on under existing cosmical conditions," as he says, he believes it "more likely that the formation of such matter and such forms took place at a time when the heat of the earth's surface was falling through those ranges of temperature at which the higher organic compounds are unstable."

Professor Huxley's opinions on the subject of Archebiosis (spoken of by him as "Abiogenesis") were very similar to those of Herbert Spencer, with the exception that he seemed more strongly opposed to the notion of its occurrence at the present day, and it is to this aspect of the question that I would now direct the reader's attention.

Why should such leaders in evolution promulgate a notion which seems to involve a quite arbitrary infringement of the Uniformity of Nature?

Both Huxley and Herbert Spencer believed that living matter originally came into being by the operation of natural causes - that is, by the unhindered play of natural affinities operating in and upon matter which had already acquired a certain degree of molecular complexity. They believed that the simpler kinds of mineral and crystalline matter continue to come in to being as they have ever done; nay, more, they believed that living matter, originally initiated by the operation of natural causes, continues to "grow" both in animal and in vegetable forms, 
solely under similar influences, and yet they considered themselves justified in supposing that natural causes are now no longer able independently to initiate this living matter, or protoplasm, as it is termed.

Professor Tyndall also took the same kind of view. Though he affirmed in the most unhesitating language the ultimate similarity between crystalline and living matter, and held that all the various structures by which the two kinds of matter may be represented are equally the "results of the free play of the forces of the atoms and molecules" entering into their composition, yet he too would have us believe that, while differences in degree of molecular complexity alone separate living from not-living matter, the physical agencies which freely occasion the growth of living matter are now incapable of causing its origination.

The opinions of these four leaders in science concerning the question of "spontaneous generation," profoundly influenced public opinion in this country during the latter third of the last century.

They were doubtless influenced, as many others have been, in the first place, by two general considerations which, taken together, seemed to carry great weight.

(I) The exposure of the untruth of certain old and crude doctrines concerning "spontaneous generation," prevalent more or less from the time of Aristotle down to the first third of the last century ; and the fact that the belief in this mode of generation has been successively driven, with increasing 
knowledge, from higher to lower forms of life, till at the last it is maintained as a mode of origin only for the very lowest and most minute of living things, has been regarded by many as one of the most weighty arguments against the de novo origin of living matter.

But this objection is robbed of all its seeming strength when it is said that the modern Evolutionist would only expect to obtain evidence concerning the de novo origin of the minutest specks of "living matter"-gradually emerging, in fluids, into the region of the visible, as opened up by a powerful microscope, and subsequently developing into the most elementary living things.

(2) The second general consideration is this. The formula omne vivum ex vivo is supposed to derive its authority from the fact that the experience of mankind generally - both skilled and unskilledtestifies to its truth. But it is almost unnecessary to say that observation is of no avail in regions where it becomes impossible, and consequently that observation cannot tell us whether previously invisible specks of living matter have arisen from invisible living germs, or by an independent process of origination. As John Stuart Mill said," "Though we have always a propensity to generalise from unvarying experience, we are not always warranted in doing so. Before we can be at liberty to conclude that something is universally true because we have never known an instance to the contrary, we must have reason to believe that if there were in nature 1 "System of Logic," 6th ed., vol. i. p. 349. 
any instances to the contrary, we should have known of them." But it was only by an utter inattention to this latter all-important proviso that the "past experience of mankind" could ever have appeared to warrant the truth of the induction omne vivum ex vivo. Living matter may have been continually coming into being all over the surface of the earth ever since the time of man's first appearance upon it; and yet the fact that no member of the human race has ever seen (or is ever likely to see) such a birth, need throw no shadow of doubt upon the probability of its occurrence.

Neither of these general considerations, therefore, however weighty they may at first seem to be, ought to have much effect in influencing the opinions of scientific men as to whether Archebiosis does or does not occur. Whatever influence they may have had in creating a kind of intellectual atmosphere favourable to disbelief, something more was needed to lead to a definite opinion against the present occurrence of anything that could be called "spontaneous generation." This finally determining element was the view taken concerning the results of experiments that had been made in relation to the question-and especially the results published in a celebrated memoir issued by Pasteur in $1862 .^{1}$

Neither Darwin, Huxley, nor Spencer ever undertook any experimental work on this subject themselves, and probably the opinions of Darwin

${ }^{1}$ Ann. de Chim. et de Physique, T. Ixiv. pp. 5-I Io. 
and Spencer were largely influenced by Huxley, who, as was shown by his Presidential Address to the British Association in 1870 , was similarly influenced by the experimental work of Pasteur.

Throughout Europe and America, indeed, upon the strength of the results and views expressed by Pasteur, there was at this time a very strong tendency to regard "spontaneous generation" as an altogether exploded doctrine. It was confidently dismissed by Pasteur both then and in succeeding years as a "chimera," and this he did on grounds which never varied from i 862 up to the date of his last writings on the subject in 1877 .

The present writer first began to work at this subject in I 869, and soon found reason for questioning the validity of this adverse verdict and recognising the lack of finality about M. Pasteur's conclusions. Professor Tyndall entered upon the question with great zeal from I $874-1877$; but he did not attempt to alter in any material way the reasons alleged by Pasteur against the occurrence of Archebiosis. To this day, therefore, and ever since i 862 , the adverse evidence has been based upon the work of Pasteur, as formulated in the memoir of that date, and repeated by him in a controversy with myself, resulting in an abortive commission of the French Academy, in 1877 .

The view that "spontaneous generation" was a "chimera," so loudly proclaimed by Pasteur and so widely echoed, was based by him upon three principal inductions from his experimental work, together with three corollaries severally deduced therefrom. 
Arranged in order, they may be stated as follows :-

Induction I.-All guarded acid fluids remain barren after they have been boiled.

Corollary.-Bacteria, Vibriones, Torulæ, and their germs are killed when they are heated in acid fluids for two or three minutes to a temperature of $100^{\circ} \mathrm{C}$.

Induction II.-Some neutral or faintly alkaline fluids, even though they are securely guarded, will ferment after they have been boiled.

Corollary.-Certain Bacteria- or Vibrio-germs are not killed by being heated for two or three minutes in fluids to a temperature of $100^{\circ} \mathrm{C}$., when these fluids have a neutral or faintly alkaline reaction.

Induction III.-All neutral or faintly alkaline guarded fluids remain barren after they have been heated for a few minutes to I $10^{\circ} \mathrm{C}$. $\left(230^{\circ} \mathrm{F}\right.$. $)$.

Corollary.-All Bacteria- and Vibrio-germs are killed, even in neutral or faintly alkaline fluids, when these are raised for a few minutes to a temperature of $110^{\circ} \mathrm{C}$.

These were the inductions and inferences to which the President of the British Association in 1870 gave his unqualified support, since it was in reliance upon Pasteur's researches and views (while ignoring those of several other workers) that he proclaimed from his Presidential Chair the doctrine omne vionm ex vivo to be "victorious along the whole line."

This cannot be considered to have been an impartial verdict, and in some of the succeeding chapters I shall have little difficulty in showing that the first and third of these inductions were not good, and that the second corollary was neither warranted nor true. That, however, if successful, would surely be found to be the breaking down of the whole of 
Pasteur's case in regard to this question-upon which he relied up to the last, and upon the strength of which the world generally has adopted his view that anything like "spontaneous generation" is a chimera. ${ }^{1}$

1 "The Life of Pasteur," by Vallery-Radot, I902, vol. ii. p. 4 I. 


\section{PART II}

THE CONDITIONS OF THE PROBLEM AND THE MODES OF EXPERIMENTATION

\section{CHAPTER VI}

EXPERIMENTAL CONDITIONS AS OPPOSED TO NATURAL CONDITIONS : THEIR UNFAVOURABLE NATURE

M ERE observation, as we have seen, can never settle the question whether Archebiosis does or does not take place at the present day. However favourable the fluid medium may be deemed, and however powerful the microscope with which we proceed to examine it, any living matter that might actually be born therein would come into existence first as ultra-microscopic particles, far too small to be recognisable. We should have particles gradually appearing in the portion of the fluid under examination, which previously were invisible. Yet this is the only mode of origin of living matter that the Evolutionist can regard as possible.

It should be obvious to all, therefore, that observation alone can never settle the problem. There are no possible means of deciding whether the ultramicroscopic particles which at last become visible have been $(a)$ actual invisible germs of some pre- 
existing organisms, or $(b)$ whether they have come into being in the mother liquid as a result of lifegiving synthetic processes.

Seeing that observation necessarily leaves the problem still unsolved, we are compelled to resort to experiment. We have to adopt, that is, certain artificial and unnatural conditions, which from their very nature must be deemed to be far less favourable for the processes resulting in life-origination than those existing in the outside world.

The unfavourable experimental conditions are two. The organic fluids with which experiment has almost always been made, in order to free them from preexisting living things, have to be subjected to degrees of heat which tend to degrade or partially decompose the organic matter they contain. Then, again, the experimental fluids are necessarily contained within glass vessels; and these are more or less impervious to the ultra-violet, or actinic, rays of light, often so potent in the bringing about of chemical combinations.

But, on the shores of oceans, in lakes, ponds, and ditches these great disadvantages would not exist, and there would be nothing to prevent the same processes of life-origination occurring in all suitable sites and media as are postulated to have occurred in the far-distant past. To think otherwise would be to doubt the uniformity of natural processes for no reason whatsoever-and that even when present conditions may fairly be deemed, as I have already indicated, much more favourable for the process than they were originally in the absence of dissolved 
organic matter derived from pre-existing living things.

The fact that the conditions for life-origination are so much more favourable in the open fields of nature than they are in our experimental vessels, would make it impossible, therefore, to deny its present occurrence, even if all experiments under strict conditions had been attended by negative results. No experiment can be regarded as trustworthy unless the vessel and its contents have been subjected to a degree of heat deemed adequate to kill all pre-existing living things that may have been contained therein-that is, unless the vessel and its contents have been reduced, as near as possible to the condition of our earth before living matter had originated on its surface.

After the initial purification by heat, we have likewise to make sure that the fluid is kept safe from external contanination - that is, from contamination by germs contained in the air.

Thus the disadvantages with which we have to contend are two-the degrading effect of the initial purifying heat process, and the partial exclusion of actinic rays by the glass vessel in which the fluid is contained - rays which might be potential, or at least helpful, in bringing about the combinations in question.

The fallacies to be guarded against are also two. We must be sure that the heat employed is adequate to kill all pre-existing living things within the experimental vessels, short of rendering the medium 
unfit for the occurrence of future processes that may lead to life-origination: this is the major difficulty, especially as preconceptions are so strong with many people that they regard the actual problem as foreclosed. If, after the purifying process, living things appear and multiply within the experimental vessels, such persons are apt invariably to attribute the fact to a survival of pre-existing germs rather than to a birth of new germs.

We must be sure also that there has been no contamination with atmospheric germs after the preliminary heating, and during the time that the experimental vessels are kept previous to the examination of their contents. But in all the fundamental experiments to be recorded by me in this work the fluids have been guarded in hermetically sealed vessels - a process which must be absolutely safe, and far more stringent than trusting to bent tubes or plugs of cotton wool as Pasteur and others have often done.

After a few words concerning the now fully admitted distribution of germs in air and water, we must address ourselves specially, and at length, to the all-important problem of the limits of vital resistance to heat, in order to obtain the necessary data as to the degree of heat really needed to sterilise the experimental vessels and their contents. If we can arrive at definite conclusions on this point, those conclusions must not be departed from except upon the basis of fresh independent evidence; an obvious rule which has been only too much and too frequently ignored in the past. 


\section{CHAPTER VII}

THE PRESENCE OF GERMS IN AIR AND WATER

$\mathrm{IT}^{\mathrm{T}}$ would be useless here to dwell at any length upon the mere speculations of Spallanzani concerning the universal distribution of germs in the atmosphere, and the subsequent experiments made by Pasteur up to the date of the publication in I 862 of his "Mémoire sur les Corpuscles organisés qui existent dans l'Atmosphère," in which he attempted to establish a foundation of facts in conformity with these speculations. At first Pasteur was inclined to believe with Spallanzani that germs existed everywhere in the atmosphere; that they were, in fact, universally diffused-though he afterwards promulgated a very modified form of this doctrine. He was compelled to surmise that they probably exist in veins or areas, variously interblended with germless regions of the atmosphere.

In addition to what were supposed to be "organised corpuscles," other fragments and foreign particles of the most varied nature were met withthough the kinds, relative proportions, and actual abundance of the different solid bodies varied extremely with the nature of the locality in which the air was examined, and also with the state and tranquillity of the atmosphere at the time. 
These investigations convinced M. Pasteur that ordinary air contains a considerable, though still a variable, number of spherical or ovoidal corpuscles, whose form and structure made him think they were organised. They varied from the smallest appreciable size up to bodies about $\frac{1}{2500}$ inch in diameter. Some of them, it was supposed, might be spores of fungi. "Mais quant à affirmer," Pasteur says, "que ceci est un spore, bien plus la spore de telle espèce déterminée, et que cela est un œuf et l'œuf de tel microzoaire, je crois que cela n'est pas possible."

He produced no direct evidence that the bodies were really germs capable of developing into one or other kind of Mould, though he says, in a note (loc. cit. p. 34), he had "originally intended to attempt this kind of proof." At this date also Pasteur did not pretend to have recognised Bacteria among the corpuscles and other débris filtered from the atmosphere, though on indirect evidence he assumed them to be present.

If this was the state of things at the time of, and for full ten years after, the publication of Pasteur's memoir, a wholly different state of knowledge now exists as a result of the labours, during the last thirty years, of an army of bacteriologists.

Common or saprophytic bacteria of different kinds undoubtedly exist in the atmosphere, though not in such numbers as were at one time supposed when surgeons performed all their operations in a mist of carbolised spray. They vary also enormously in different localities-- being less abundant in country districts than in towns; diminishing again rapidly 
in their abundance as mountain elevations are ascended, or over the sea as we recede from the land. " Pathogenic bacteria, on the other hand, are only occasionally present in the air. The practical results obtained from the examination of air for pathogenic bacteria have been slight. We know that at times they must be in the air, but unless we purposely increase their numbers they are so few in the comparatively small amount of air which it is practicable to examine that we rarely find them. Examination of dust, however, in hospital wards and sick-rooms, has revealed tubercle bacilli and other pathogenic bacteria." I

Much the same kind of thing has to be said concerning waters of different kinds; they all contain various kinds of Bacteria : even the purest waters are not free from them, and ordinary tap waters contain them in abundance. River water becomes contaminated by washings from the soil after rains, as well as from the contents of drains and sewers. The results of such contamination are fortunately rendered less disastrous by the fact that a natural process of purification occurs tending to diminish the evil. Thus Park says (loc. cit. p. 449): "That river water which has been fouled by sewage will, in the course of a few miles, through the dilution of additional supplies, through sedimentation, and through oxidation, become greatly purified is an indisputable fact."

But in reference to experiments on the question of the possible occurrence of Archebiosis we need

${ }^{1}$ Park's "Pathogenic Micro-organisms," 2nd ed., 1 906, p. 448. 
not concern ourselves at all minutely with the number of the organisms existing either in air or in water. Nor need we trouble ourselves to enquire how many different kinds of organisms are to be found in the air. In these experiments we find, as a rule, within our experimental vessels only different varieties of Bacteria, and of Torulæ, or other Fungus germs, such as are shown in Plate I. $^{1}$

We know perfectly well that these organisms are apt to be found in variable quantity in the air, or in the water that may be contained in our experimental vessels. So that an initial purifying heat, to which the experimental vessels and their contents are submitted, is had recourse to solely with the view of killing all such pre-existing forms of life. Though we try to exclude them as far as possible, it is practically immaterial, when we are operating with hermetically sealed vessels, whether a few germs may be contained therein or not. We have to strive to kill all that may be there; so that, before proceeding with such experiments, the most careful consideration must be given to all available evidence bearing upon the question of the destructive influence of heat upon different kinds of living matter, and especially upon the above-mentioned micro-organisms and their germs.

1 In this Plate, Fig. I, A, shows some very small Bacteria developing from minute particles; while in $\mathrm{P}$, larger and stained Bacilli, from a hay infusion, are represented. 


\section{CHAPTER VIII}

THE LIMITS OF VITAL RESISTANCE TO HEAT: EARLY

OBSERVATIONS

THE all-important question of the destructive influence of heat upon living matter requires to be looked at from a broad point of view, because of the essential unity of protoplasm. As Huxley said," "Beast and fowl, reptile and fish, mollusc, worm, and polype, are all composed of structural units of the same character, namely, masses of protoplasm with a nucleus. ... What has been said of the animal world is no less true of plants. ... Protoplasm simple or nucleated is the formal basis of all life. ... Thus it becomes clear that all living powers are cognate, and all living forms are fundamentally of one character." If, therefore, we can show that the limits of vital resistance to heat for very many different representatives of the animal and the vegetal kingdoms fall within comparatively narrow limits, we shall be doing our best to establish a sound induction concerning the thermal deathpoint of living matter generally-and thus to discredit the vague or extravagant statements that are apt to be made on this subject.

In the latter third of the eighteenth century a 1 "Lay Sermons," pp. I26-I29. 
controversy was going on between the acute and learned Abbé Spallanzani and our own countryman Tuberville Needham on the question of "spontaneous generation," and Spallanzani is the only one espousing his side of the question who has fairly and fully faced the question of the degree of heat which proves fatal to various living things, by making it the subject of direct investigation. Other workers have more or less distinctly confounded the issues of this question with that of the cognate though distinct problem, as to whether certain infusions could themselves prove mother liquids and give independent birth to living matter.

The tendency with Pasteur, with Tyndall and others has been altogether to ignore this latter possibility - to regard it as a "chimera," and as a problem not to be seriously considered." It was wholly different, however, with Spallanzani. In some of his own experiments he had found living organisms in infusions which had been heated for half-an-hour in closed vessels. And, if no good reason could be found for supposing that the experimental results referred to were to be accounted for by a "survival of germs," he confessed he must admit the fact of an independent and germless origin

1 Pasteur, for instance, said (Compt. Rend., July 23, 1877, p. I79) : "Dans ces sortes d'études, le résultat positif est celui qui ne donne pas d'organismes, et le résultat negatif est celui où l'on en rencontre." While in the previous month, in a very positive and dogmatic letter which appeared in the Times (June IS, I877), Tyndall wrote: "Dr Bastian says that two interpretations of my facts are equally admissible. $\mathrm{He}$ is again wrong; there is but one interpretation possible. An interpretation which violates all antecedent knowledge is no interpretation at all." 
of living things. This simple issue was therefore fully realised by Spallanzani, and, acting in accordance with the most obvious of scientific principles, he carefully sought for fresh evidence, by means of welldirected experiments, in order to guide him towards a conclusion as to whether germs of living things could or could not have resisted the action of boiling water for more than half-an-hour.

His results were embodied in a work, translated by Jean Senebier under the title "Opuscles de Physique Animale et Végétale" (Geneva, I767), to which reference may most conveniently be made.

Spallanzani approached the question in the following manner: "Can one," he says, "find any proof sufficient to banish, or, at all events, to diminish one's natural repugnance to admit that the germs of animalcules of the lowest order have the power of resisting the action of boiling water? In reasoning from the germs or eggs of animals with which we are acquainted, would it be difficult for us to imagine animalcules having this peculiarity? It is true that we are not acquainted with any eggs endowed with such properties. I have already considered this subject in the ninth chapter of my Dissertation. I there show how several kinds of eggs of insectsnot to speak of eggs of birds-perish under a heat less than that of boiling water. I have shown also that the seeds of plants are destroyed when they are exposed to the heat of boiling water, and that even those whose coat is of the hardest description are not thereby spared."

But, he goes on to say, as he had only been able 
hitherto to make his observations on a limited number of eggs and seeds, there was the chance that more extended observations might reveal some which were capable of resisting this generally destructive influence. He says he had never lost his hopewith regard to seeds more especially-since he had seen a statement by Duhamel to the effect that some grains of wheat had germinated after having been heated in a stove to a temperature above the boiling point of water. ${ }^{1}$ And as there is a considerable resemblance between seeds and eggs, Spallanzani was led to hope that something of the same alleged extraordinary capacity for resisting heat might be possessed by the eggs or germs of such organisms as make their appearance in previously boiled fluids. He was therefore stimulated to undertake fresh observations upon eggs and seeds generally, with the view, on the one hand, of ascertaining the precise temperature which proved fatal to each kind; and, on the other, of finding out whether these eggs or seeds were capable of resisting a greater degree of heat than the several animals or plants to which they belonged.

In carrying out these inquiries Spallanzani adopted the following method (loc. cit. p. 53): He placed the eggs, seeds or organisms made use of in his

${ }^{1}$ Heated in all probability in the dry state. But it is well known that seeds and desiccated lower animals can resist the influence of heat much better in the dried state than when they are thoroughly moistened and then heated; and it is as to the effects of heat upon living matter under the latter conditions that we are at present concerned-for to such conditions the living matter will always be exposed in our experiments. 
experiments in a vessel containing cold water, within the upper strata of which was immersed the bulb of a thermometer. The water was then heated slowly, and when the thermometer indicated that the temperature had been attained whose effects it was desired to test, the eggs, seeds, or organisms were at once withdrawn and placed, under suitable conditions, in a separate vessel where their subsequent fate could be watched. The effects of different grades of heat upon the objects experimented with were thus estimated, and the temperature in successive trials was mostly made to differ from that last employed by $5^{\circ} \mathrm{R}$. - that is, about I I ${ }^{\circ} \mathrm{F}$.

We need only refer to a few of the results obtained by Spallanzani. Silk-worms' eggs and the eggs of the Elm-moth developed less and less frequently when successive batches were heated to temperatures approaching $144 \frac{1}{2}^{\circ} \mathrm{F}$. When they were actually submitted to this heat all perished, though the highest temperature followed by development is not recorded. Silk-worms themselves, as well as the caterpillars of the Elm-moth, were uniformly killed as soon as the water in which they were immersed attained $108 \frac{1}{2}^{\circ} \mathrm{F}$. Eggs of the common Blow-fly also only developed in very small numbers when raised to the temperature of $135^{\circ}$; all perished at $140^{\circ}$; and larvæ reared from the eggs perished, as those of the Silk-worm and Elmmoth had done, as soon as the temperature of the water rose to $108 \frac{1}{2}^{\circ}$. Certain aquatic organisms, such as Leeches, Nematode and other Worms, 
together with Water Fleas, were found to be killed at or about I IO $^{\circ} \mathrm{F}$.

Experiments were also made with the seeds of the Chick-pea, Lentil, Wheat-grass, Flax and Clover. The mode of procedure was as before, so that there was only a momentary exposure to the temperatures about to be cited. Of those which had been exposed to $190^{\circ} \mathrm{F}$. many did not germinate; still fewer of the seeds that had been exposed to $201^{\circ}$ produced young plants; while of those heated to $212^{\circ}$ not one germinated. After the young plants which had been developed from seeds heated to lower temperatures had grown for thirteen days their capability of resisting heat was tested in the manner described (that is, by only immersing the roots of the plants in water, while the temperature was being raised), and with the following results. Those whose roots had been momentarily exposed to $156^{\circ}$ continued to live after they had been replanted; while others whose roots had been exposed to $167^{\circ}$ and upwards speedily dried up and perished, though all alike had been carefully replanted in well-watered earth. Spallanzani's method here was defective. The whole of the plant, in each case, ought to have been immersed in the water; and had this been done they would probably have succumbed at very much lower temperatures, as Sachs has since shown.

This investigator says," "I have convinced myself that a considerable number of plants are 1 "Text-Book of Botany," transln., 1875, p. 650. 
killed by an immersion for only ten minutes in water of $45^{\circ}$ or $46^{\circ} \mathrm{C}$. [ [1 $33^{\circ}$ or $1148^{\circ} \mathrm{F}$.]."

Thus Spallanzani's researches taught him three things: (I) that eggs can withstand a decidedly higher degree of heat than that proving fatal to their parents; (2), that an analogous difference exists between seeds and plants, in respect to their capacity of withstanding the action of heat; and (3), that seeds and plants can resist higher grades of heat than eggs and animals respectively.

In seeking for an explanation of these results, he quickly dismissed-as Burdach did rather laterthe utterly improbable notion that the smallness of the germ or egg can act as its safeguard, by rendering it less amenable to the influence of heat. $\mathrm{He}$ inclined rather to the view that the increased power of resistance possessed by seeds and eggs, as compared with the organisms from which they proceed, was due to the simplicity of the embryo within the seed or the egg; and he asks whether the fact of this life being "so small and so feeble"-being "a life which deserves so little the name of life "-may not be the reason that seeds and eggs are able to resist heat so much better than developed organisms. $\mathrm{He}$ adduces reasons tending to support this view; and thinks the same kind of explanation is applicable to the greater tolerance of the injurious effects of heat upon seeds and plants, as compared with that shown by eggs and animals.

The greater tenacity of life of seeds is only in part due to the fact that the outer coats of most seeds are much harder than those of eggs. Thus the 
envelopes of some seeds which are only killed at a temperature near 2 I $2^{\circ}$ are not harder than the shell of an egg, which is nevertheless killed at the much lower temperature of $140^{\circ}$. The actual difference is explicable principally, according to Spallanzani, rather by the fact that the fluids contained within the egg are so much more abundant than those within the seed.

Spallanzani's argument thus naturally suggests the notion that many of the seeds with which he experimented required a high temperature to kill them, merely on account of their dryness. If the seeds had previously been well soaked in cold water, or better still in warm water, so as to have been thoroughly moistened, might they not have been killed at a much lower temperature-that is, only a little, if at all, over $140^{\circ} \mathrm{F}$.- the temperature which proved destructive to the more moist animal germs?

It is well known, in fact, that seeds of plants provided with a thick and hard coat may-especially after prolonged periods of desiccation-germinate even after they have been boiled for a very long time in water. This was ascertained by Pouchet to be the case with an American species of Medicago. Some of the seeds were completely disorganised by the boiling water, while a few remained intact, and it was these latter which were afterwards found to germinate. They had been protected from the influence of the boiling water by their very dry and hardened coats. Facts of the same kind have been recorded by Professor Jeffries Wyman, who pointed 
out the slower action of cold as compared with hot water in moistening dried seeds of other Leguminosæ. When such seeds had been previously moistened, a brief exposure, he says, to "the action of boiling water has been found uriformly fatal." He adds, "All the organisms in which we are interested at present, however, have no such protection. These are mere specks or masses of protoplasm, which are either naked or provided only with thin coverings" -reference being here made to germs of Bacteria and Fungi, with which we shall be concerned in experiments concerning life-originătion.

Thus, not a single living thing, egg, or seed could be shown by Spallanzani to be capable of resisting, when in the moist state, an exposure to boiling water for a single moment; and in order not to accept the conclusions of Needham he was obliged to fall back upon two assumptions: $(a)$ that the unknown germs, whose existence he postulated, were of the nature of seeds rather than eggs, being supposed to be capable of undergoing desiccation with impunity, and thus possessing a greater power of resisting heat; and $(b)$ that although no seeds could be shown to be able to resist the influence of boilingwater, these unknown seed-like germs might be able to do so.

But Burdach exhibited much sagacity some years later when in reference to unknown germs, postulated also in his time, he said: "Les dit-on trop petits pour être aperçus, c'est avouer qu'on ne peut rien savoir de leur existence. . . . Croire que partout où l'on rencontre des infusoires, ils ont été précédés 
d'œufs, c'est donc admettre une pure hypothèse, qui n'a d'autre fondement que l'analogie. . . . Si c'est seulement par l'analogie qu'on suppose des œufs chez eux, il faut accorder à ces œufs des propriétés semblables à celles de tous les œufs connus : car ce serait jouer sur les mots que de supposer qu'ils en ont de particulières à eux seuls." 1

1 "Traité de Physiologie," Transl. I837, t. i. p. 22. 


\section{CHAPTER IX}

THE LIMITS OF VITAL RESISTANCE TO HEAT : LATER

OBSERVATIONS

SO far we have been referring to the influence of $S$ heat upon living matter when it is suddenly applied to an altogether unaccustomed extent. This is the mode of operation with which we are specially concerned; since with a view to the interpretation of experiments on the origin of life question, we wish to know the effects of great heat suddenly applied upon organisms or their germs that have been accustomed to ordinary atmospheric and aquatic temperatures.

On the other hand, it should be pointed out that Confervæ. and other low algoid organisms have been found living in hot springs at comparatively high temperatures; although the very highest of the recorded temperatures of these hot springs is still a few degrees below the boiling-point of water. The various observations made upon this subject have been collected, and criticised with much care by Professor Jeffries Wyman. ${ }^{1}$ The highest temperatures cited which are at all trustworthy in which Confervæ or allied organisms have been met with are thus summarised by him:-- "The statements

1 American Journal of Sicience and Arts, vol. xliv., Sept. I 867. 
we have quoted give satisfactory proof that different kinds of plants may live in waters of various temperatures as high as $\mathrm{I} 68^{\circ} \mathrm{F}$., as observed by Dr Hooker in Sorujkund; $174^{\circ}$, as observed by Captain Strachey, in Thibet; $185^{\circ}$, as observed by Humboldt in La Trinchéra; $199^{\circ}$, as observed by Dr Brewer in California; and $208^{\circ}$, as observed by Decloizeauz in Iceland."

Having no grounds for criticising these observations, we are bound to look upon them, provisionally, as correct, and taken with all due care-though it is only fair to add that Max Schultze ${ }^{1}$ and F. Cohn appear to be not altogether satisfied with some statements of the same kind. Such instances, if thoroughly accurate, may perhaps be taken as examples of the highest temperature that it is possible for living matter to endure, day after day and week after week, even where it has been inured to the influence of heat in the most gradual manner. The real point of view from which such facts should be regarded was pointed out by Professor Wyman when he said: "Having become adapted through a long series of years to their surroundings, such organisms may be supposed to live under circumstances the most favourable possible for sustaining life at a high temperature. It is a well-known physiological fact, that living organisms may be slowly transferred to new and widely different conditions without injury; but if the same change is suddenly made they perish. In the experiments made in our laboratories, the change of conditions is 1 "Das P'rotoplasma," Leipzig, $\mathrm{I} \$ 63$, p. 67. 
relatively violent, and therefore liable to destroy life by its suddenness.'

Hence it is, as we have seen from the experiments of Spallanzani, and shall now see from other more recent investigations of a similar nature, that a temperature considerably lower than that of the hot springs above mentioned suffices to kill almost all living matter not previously inured to the influence of heat.

Thus Pouchet ${ }^{1}$ found that all kinds of Ciliated Infusoria experimented with were killed at $13 \mathrm{I}^{\circ} \mathrm{F}$.; and while confirming this observation, I have found that a brief exposure to the same temperature always sufficed to kill Amœbæ, Monads, Euglenæ, Desmids, Rotifers, Nematoids, and other minute aquatic organisms. I did not try to ascertain what was the lowest temperature which would prove fatal to these organisms, though many of the same kinds of living things were found by Spallanzani to perish at temperatures from $107^{\circ}$-II $3^{\circ}$; while Max Schultze and Kühne (in part working over the same ground) subsequently fixed the heat-limits fatal to such organisms at temperatures varying between $104^{\circ}$ and $\mathrm{II}_{3}^{\circ} \mathrm{F}$. The protoplasm entering into the formation of the tissue elements of higher animals was not only killed at a point below the higher of the two just named, but it became coagulated also, and assumed the condition named by Kühne, "heat-stiffening."

Both Max Schultze and Kühne also found that the protoplasm of plant-cells with which they ex1 "Nouvelles Expériences," etc., I864, p. 38. 
perimented (belonging to the genera Urtica, Tradescantia, and Vallisneria) was similarly killed and altered by a very brief exposure to a temperature of I $8 \frac{1}{2}^{\circ} \mathrm{F}$. as a maximum.

We now have to approach the problem to which the other investigations already cited are to be regarded as necessary and illuminating preliminaries, We have to ascertain, that is, $(a)$ the limits of resistance to heat, when in the moist state, of Bacteria and Torulæ; and $(b)$ the more difficult problem of the resistance to heat under similar conditions of germs of Bacteria and Fungi, both before and after they have been submitted to desiccation-Bacteria or Moulds (such as are shown in Plate I.) being the organisms that are prone to appear within our experimental vessels, and the secret of whose origin we are striving to discover.

\section{The Thermal Death-point of Bacteria and Fungus-germs in the Moist State}

It has now been very definitely ascertained that certain fluids exist which, after they have been boiled, never even seem to give birth to Bacteria, although they continue to be quite suitable for the support and active multiplication of any such organisms that may subsequently have been added to them. Among such fluids I may name one commonly known as "Pasteur's solution," and also a much simpler one, which I have myself more commonly used, consisting merely of ten grains of neutral ammonium tartrate and 
three grains of neutral sodium sulphate to an ounce of distilled water. When portions of either of these fluids are boiled in previously superheated flasks, they will continue quite clear for many days, or even for weeks. Even when the narrow necks of the flasks containing them remain open, these fluids will often not become clouded or turbid, and on microscopical examination no living Bacteria are to be detected therein.

Yet, in order to show that such fluids are still thoroughly favourable media for the multiplication of Bacteria, all that is necessary is to bring either of them into contact with a glass rod previously dipped into a fluid containing swarms of such organisms. In about thirty-six hours after this has been done (the temperature being about $80^{\circ} \mathrm{F}$.), the fluid, which had hitherto remained clear, becomes clouded and turbid owing to the rapid growth and multiplication of Bacteria-as a microscopical examination will reveal.

Facts of this kind were shown by Burdon Sanderson ${ }^{1}$ to hold good for portions of boiled "Pasteur's solution," even when freely exposed to the air ; and I have often verified them with my ammonium tartrate solutions.

If, however, a freshly prepared infusion of turnip is made, and this fluid after filtration is boiled, poured into a superheated flask, and left standing side by side with the ammonium tartrate solution, at a temperature of $80^{\circ}$ to $90^{\circ} \mathrm{F}$., a remarkable difference between

1 Thirteenth Report of the Medical Officer of the Privy Council (187 I), p. 59. 
the two fluids is speedily shown. The latter, as we have seen, remains clear, while the turnip infusion almost invariably becomes turbid in the course of two or three days, owing to the presence and multiplication of myriads of Bacteria.

Now, all the experimental work to be detailed in this volume has been undertaken in order to throw light upon the cause of this different behaviour of the turnip infusion, and other fluids which behave in a similar manner. It is clear that both are nourishing fluids, since we find that Bacteria are capable of growing and multiplying in each of them. What we want to know is, whether the turnip infusion, and other fluids that behave in a similar manner, are also generating fluids - that is, fluids capable, after they have been boiled, of giving birth to specks of living matter which speedily take the form of Bacteria or Torulæ. So far we have found no evidence showing that any living thing could survive even momentary exposure to the influence of boiling water, so that the problem is a most legitimate one.

The latter possibility, however, is one which has been utterly repudiated both by Pasteur and by Tyndall, as we have seen (p. 33, note). In his celebrated memoir published in 1862 , one of Pasteur's principal objects was to determine whether fermentation could or could not take place without the intervention of living organisms which he, at that time, held (in opposition to many other chemists) to be the only true ferments. In Chapters iv. and v. of his memoir he records experiments in which he inoculated fluids with dust filtered from the atmos- 
phere, which contained, as he was well aware, a large amount of what those who differed from him regarded as not-living ferment (mere organic matter), while possibly there existed living Bacteria or their germs-for at this date none such had ever been definitely discovered in the atmosphere. In explaining the results of his experiments, however, M. Pasteur thought he was pursuing a logical and scientific method when he attributed the fertilising results obtained to the action of the possibly existing elements (Bacteria) in the inoculating compound; while he ignored altogether the other element (mere organic matter) which was certainly present in comparatively large quantities.

As a very able writer said in an article on "The Germ Theory and Spontaneous Generation," in the Contemporary Review for April I877:-“Once assume as a starting-point [the truth] of the germ theory, and the ascertainment of the death-point of bacterium germs is the simplest thing in the world. If life appears in the fluid inoculated with them, say at once that it is due to the germs, and that the heat was not enough to kill them. If, on the other hand, the fluid remains barren after the germs have been introduced, it follows that the heat was fatal. For the purpose of testing the validity of the germtheory, it is obvious that no such petitio principii can be for a moment admitted; and yet, oddly enough, it was only by this deliciously naive reasoning that Pasteur supported his view of the vital resistance of bacterial germs. He found that one or two acid fluids with which he worked would not 
putrefy after being heated to the boiling-point, and he inferred (so far legitimately enough) that no germ could (in such a fluid) sustain the temperature of $100^{\circ} \mathrm{C}$. He also found that one or two neutral or faintly alkaline fluids would putrefy after boiling, though they failed to do so if heated to $110^{\circ}$ or even $105^{\circ} \mathrm{C}$. He thereupon straightway assumed that putrefaction could not come without germs, and therefore declared that germs heated in these nonacid fluids could survive a temperature of $100^{\circ} \mathrm{C}$., though they succumbed to a slightly greater heat. Of course the inference was idle while the germ theory was in question; and some independent method of fixing the death-point remained to be discovered, if even it was to be fixed at all."

In 187 I I made the first attempt to ascertain the exact death-point of Bacteria, when heated in the nourishing neutral solution of ammonium tartrate and sodium phosphate. The details of the mode of experimentation have been given elsewhere. ${ }^{1}$ It is only necessary to say here that specimens of this fluid, inoculated with a drop of a similar fluid swarming with Bacteria, were heated for about ten minutes to the following temperatures, $\mathrm{I} 22^{\circ} \mathrm{F}$., I $3 \mathrm{I}^{\circ}$, $140^{\circ}$, $149^{\circ}$, $158^{\circ}$, and $167^{\circ}$, and the fluids in hermetically sealed vessels were subsequently maintained at a temperature of about $90^{\circ} \mathrm{F}$.

The results were as follows:-The flasks whose contents had been heated to $\mathrm{I}_{2} 2^{\circ}$ and $\mathrm{I} 3 \mathrm{I}^{\circ}$ respectively began to exhibit an opalescent tinge in

1 "Modes of Origin of Lowest Organisms," 1 S7 I, pp. 51-56. 
the contained fluids after the first or second day; and after two or three more days, the fluid in each became quite turbid and opaque, owing to the presence and multiplication of myriads of Bacteria, together with a smaller number of Torula. But the fluids in the flasks which had been exposed to the higher temperatures of $\mathrm{I} 40^{\circ}$, I $49^{\circ}$, I $58^{\circ}$, and I $67^{\circ} \mathrm{F}$., showed not the slightest trace of turbidity, and no diminution in the clearness of the fluid while they were kept under observation-that is, for a period of twelve or fourteen days.

One kind of conclusion only was to be drawn from these experiments, the conditions of which were in every way similar, except as regards the degree of heat to which the inoculated fluids were subjected-seeing that the organisms were contained in a fluid which had been proved to be eminently favourable for their growth and multiplication. If those inoculated fluids, which have been raised to $122^{\circ}$ and $\mathrm{I} 3 \mathrm{I}^{\circ}$ for ten minutes, are found in the course of a few days to become turbid, obviously the organisms cannot have been killed by such an amount of exposure to heat; while if similar fluids, similarly inoculated, which have been raised to temperatures of $140^{\circ}, 149^{\circ}$, I $58^{\circ}$, and $167^{\circ} \mathrm{F}$., remain sterile, such sterility seemed only explicable by the supposition that the multitudes of Bacteria and Torulæ, introduced into the nourishing solutions, have been killed by exposure to these temperatures. Thus $140^{\circ} \mathrm{F}$. $\left(60^{\circ} \mathrm{C}\right.$.) was shown to be the thermal death-point of Bacteria and Torulæ.

Somewhere about the same time Baron Liebig 
arrived at a similar conclusion concerning Torule. He said," "A temperature of $60^{\circ} \mathrm{C}$. kills the yeast cells; after exposure to this temperature in water, they no longer undergo fermentation, and do not cause fermentation in a sugar solution. . . . In like manner active fermentation in a saccharine liquid is stopped when the liquid is heated to $60^{\circ} \mathrm{C}$., and it does not recommence on cooling the liquid."

In the following year Professor Cohn and Dr Horwath made experiments almost similar to mine in regard to the death-point of Bacteria, but not of Torulæ. It does not appear that they were then aware of my prior investigations. They, however, arrived at results almost precisely similar, as may be seen when Professor Lohn says": "These experiments demonstrated, without exception, that no Bacteria were developed in the flasks which were kept at a temperature of $60^{\circ}-62^{\circ} \mathrm{C}$. for an hour, and that the contained fluid remained clear; on the other hand, flasks containing bacterial fluid which had only been heated to $50^{\circ} \mathrm{C}$. or $40^{\circ} \mathrm{C}$. became clouded in consequence of the multiplication of Bacteria, in a time ranging from two to three days."

In the year $1873 \mathrm{I}$ returned to this subject, in order to ascertain whether Bacteria would be killed at precisely the same temperatures in organic infusions as they had been in a neutral saline solution. The question was how to operate with such

1 Translation of a paper on "Alcoholic Fermentation," in Pharmaceutucal Journal, July 30, i 870 , p. 8 I.

2 "Beiträge zur Biologie der Pflanzen," I\$72, p. 2 I9. 
fluids in order to prevent all chance of their being "generating," as well as "nourishing," fluids. A clue in this direction had been met with two years previously, after dealing with the death-point in the saline solution, which is thus referred to ${ }^{1}$ :- "If, on the same slip, though under different cover-glasses, specimens of a hay infusion, turbid with Bacteria, are mounted $(a)$ without being heated, $(b)$ after the fluid has been raised to $122^{\circ} \mathrm{F}$. for ten minutes, and (c) after the fluid has been heated to $140^{\circ} \mathrm{F}$. for ten minutes, it will be found that in the course of a few days the Bacteria under $a$ and $b$ have notably increased in quantity; while those under $c$ do not become more numerous, however long the slip is kept." Facts of a similar kind were also found at this period when a turnip infusion and an impure neutral saline fluid was dealt with. The multiplication of Bacteria beneath the cover-glass, when it occurs, is soon rendered obvious even to the naked eye by the increasing cloudiness of the film.

Under (c) there seemed to be clearly no origination. Then, again, it was pointed out by Gruithuisen, early in the last century, that many infusions, otherwise productive, ceased to be so when they were poured into a glass vessel whilst boiling, and when this was filled, so that the tightly fitting stopper touched the fluid. Having myself proved the truth of this assertion, and ascertained that under such conditions infusions of hay and turnip acted only as " nourishing" fluids, the mode of experimentation 1 "Modes of Origin of Lowest Organisms," I87 I, p. 60. 
became easy. Full details as to the method adopted and the results obtained are given in The Proceedings of the Royal Society (March 1873, pp. 226-32).

It is only necessary to say here that turnip and hay infusions were boiled for ten minutes, and after they had become cool they were inoculated with a hay infusion swarming with Bacteria in the proportion of one drop to each ounce of the fluid. This stock liquid was then poured into a purified beaker placed upon a sand-bath, and its contained fluid (in which a thermometer was immersed) was gradually raised to the required temperature, at which it was maintained for five minutes, by alternately raising the beaker from and replacing it on the sand-bath. Purified one-ounce bottles and corks were then used, the former being filled to the brim, and the corks tightly pressed home. The bottles, filled with inoculated infusions heated to various degrees, were subsequently maintained at a temperature ranging from $65^{\circ}$ to $75^{\circ} \mathrm{F}$.

The results of the IO2 experiments that were then made with hay and neutral acid turnip infusions were embodied in two tables (loc. cit., p. 230), an inspection of which led to the following conclusions :"The experimental results above tabulated seem naturally divisible into three groups. Thus, when heated only to $\mathrm{I} 3 \mathrm{I}^{\circ} \mathrm{F}$, all the infusions became turbid within two days, just as the inoculated saline solutions had done. Heated to $5^{\circ} \mathrm{F}$., all the inoculated organic infusions remained clear, as had been the case with the saline solutions, in my previous experiments, when heated to $140^{\circ} \mathrm{F}$. 
There remains, therefore, an intermediate heat-zone (ranging from a little below $140^{\circ}$ to a little below I $58^{\circ} \mathrm{F}$.), after an exposure to which the inoculated organic infusions are apt to become more slowly turbid, although inoculated saline solutions raised to the same temperature invariably remained unaltered."

The cause of this difference was later on dealt with in another communication to the Royal Society. It is unnecessary, however, to go into this question now, since it is at present generally admitted by bacteriologists that Bacteria and Torulæ in their active state, as they are found growing and multiplying in fluids, are invariably killed by a brief exposure to temperatures ranging between $140^{\circ}$ and $158^{\circ} \mathrm{F}$. $\left(60^{\circ}-70^{\circ}\right.$ C. $)$-and for the most part nearer to the former than to the latter limit.

Then, again, besides Bacteria and Torulx, the bodies which are prone to show themselves at times in experimental vessels raised to much higher temperatures are ordinary Moulds. And in regard to such organisms Sachs says ${ }^{1}$ : "Of ninety-four experiments which were made by Tarnowski with all possible precautions, the result was that the spores of Penicillium glaucum and Rhizopus nigricans... heated in their proper nutrient fluids nevertheless entirely lost their power of germination at $54^{\circ}$ or $55^{\circ}$ C. $\left[\mathrm{I}_{3} \mathrm{I}^{\circ} \mathrm{F} .\right]^{\prime} "$

So far then the various experimental results upon the question of "vital resistance" to heat have yielded remarkably harmonious results. There is still another side to the question, however, which 1 "Text-book of Botany," Transl. I875, p. 65 I,

E 
must be dealt with in another chapter, before we can enter upon the question as to whether, in addition to mere "nourishing" fluids, there are actual "generating" fluids, in which germs of Bacteria, of Torulæe, and of Moulds may arise de novo. 


\section{CHAPTER $\mathrm{X}$}

THE LIMITS OF VITAL RESISTANCE TO HEAT: CONCLUSION

$A^{T}$ the commencement of the last chapter I had A to refer to an exceptional group of organisms - the Confervæ found in hot springs-which are capable of carrying on their very simple life-processes under conditions of temperature that have been found to be fatal to almost all other forms of life. And now I have to refer to an exceptional group of Bacteria--the so-called "Thermophilic Bacteria" which are capable of carrying on their simple lifeprocesses at temperatures that would certainly prove fatal to many of their allies. These organisms have only been at all well known during the last ten years, the most extensive information concerning them being contained in two memoirs by Drs. Macfadyen and Blaxall. ${ }^{1}$

They say their researches have led them to the conclusion "that there is a widely distributed group of organisms in nature which find their optimum temperature of growth between $55^{\circ}$ and $65^{\circ} \mathrm{C}$." Fourteen different forms have been studied and described by these investigators. They were all

1 Journal of Pathology and Bacteriology, Nov. 1894, and Transactions of Jenner Institute of Preventive Medicine, second series, I899. 
Bacilli, for the most part motionless, and were found principally in surface and deep layers of the soil, in Thames mud, in sewage water, in the intestines of many kinds of animals and in their dejecta, also in all samples of ensilage examined. Rabinowitsch and other observers have also found such organisms in similar sites.

The temperatures most favourable to their growth were tested by our authors, with three different cultivating media for all the fourteen forms. Some grew well at temperatures ranging from $37^{\circ}$ to $47^{\circ} \mathrm{C}$. This was nothing new, as thirty years ago I had made known that Bacilli would multiply most freely at $50^{\circ} \mathrm{C}$. in neutralised urine. But Macfadyen and Blaxall go on to say: "We may, however, judging by our observations, take $55^{\circ}$ to $65^{\circ} \mathrm{C}$., and even to $67^{\circ} \mathrm{C}$., as an optimum temperature, both as regards the quickness and the amount of growth that occurred. It was interesting to note that in one or two instances a very good growth occurred at $72.5^{\circ} \mathrm{C}$., whilst in a few cases evidence of growth was obtained even at $74.5^{\circ} \mathrm{C}$. . . At the same time there was no doubt about the optimum temperature being about $50^{\circ} \mathrm{C}$." Under cultivation the majority of the Bacilli showed a tendency to grow in long chains. Only two out of the fourteen forms showed undoubted motility, though many of them developed "spores"--bodies about which, in other Bacilli, we shall have much to say presently.

Many of these thermophilic organisms were capable of growth through a remarkable range of temperature, though certainly many of them, outside 
the laboratory, would not often have found the higher temperatures with which experiments were there made. And, as the authors say, it is only "reasonable to suppose that such a widely distributed group of organisms must find favouring natural conditions outside the factor of temperature, these conditions being probably of a chemical nature."

Although some of these thermophilic Bacteria are undoubtedly capable of flourishing at temperatures that prove rapidly fatal to other Bacteria, they are not so remarkable in this respect as the Confervæ referred to in the last chapter, many of which flourished at even higher temperatures. We have still much to learn concerning each of these groups of organisms of lowest grade; but as we are distinctly told by Macfadyen and Blaxall that the thermophilic Bacteria were never detected in tap water (and as they would be still less likely to be found in distilled water with which the most important experiments to be recorded in this work have been made), they may, from our point of view, be almost as much disregarded as the Confervæ of the hot springs. Neither of these exceptional organisms are, in fact, at all likely to complicate our work.

Seeing that in the experiments with saline and organic infusions recorded in the last chapter the solutions were inoculated with a drop of a fluid in which either Bacteria alone, or Bacteria and Torulæ, were multiplying rapidly, we must suppose that they were multiplying in their accustomed manner, as 
much by the known method of fission as by any unknown and assumed method of reproduction. As I then said: "These experiments seem to show, therefore, that even if Bacteria do multiply by means of invisible gemmules as well as by the known process of fission, such invisible particles possess no higher power of resisting the destructive influence of heat than the parent Bacteria themselves possess." 1

Nothing more than this could be said in IS7I; though five years later memoirs were published by Professor Cohn ${ }^{2}$ and Dr Koch making known the existence of easily visible "spores," both in hay and in anthrax Bacilli. When these organisms are cultivated at blood-heat, and the infusions are exposed to air filtered through a cotton-wool plug, both these forms of Bacilli tend to grow into long interlacing threads at the surface of the fluid; and in twenty-four to forty-eight hours a number of highly refractive particles appear at short distances from one another within the threads-which are the "spores" in question. Subsequently, similar bodies have been found in many other Bacilli and, among them, in the thermophilic Bacilli to which reference has recently been made.

It remains, therefore, to ascertain what amount of heat these spores are capable of withstanding, first of all $(a)$ when in their moist state, and subsequently $(b)$ after they have undergone desiccation.

1 "Modes of Origin of Lowest Organisms," I S7 I, p. 60.

2 "Bcitr. zur Biologic der Pflunzcn," $2^{\text {er }}$ Bd., p. 268. 

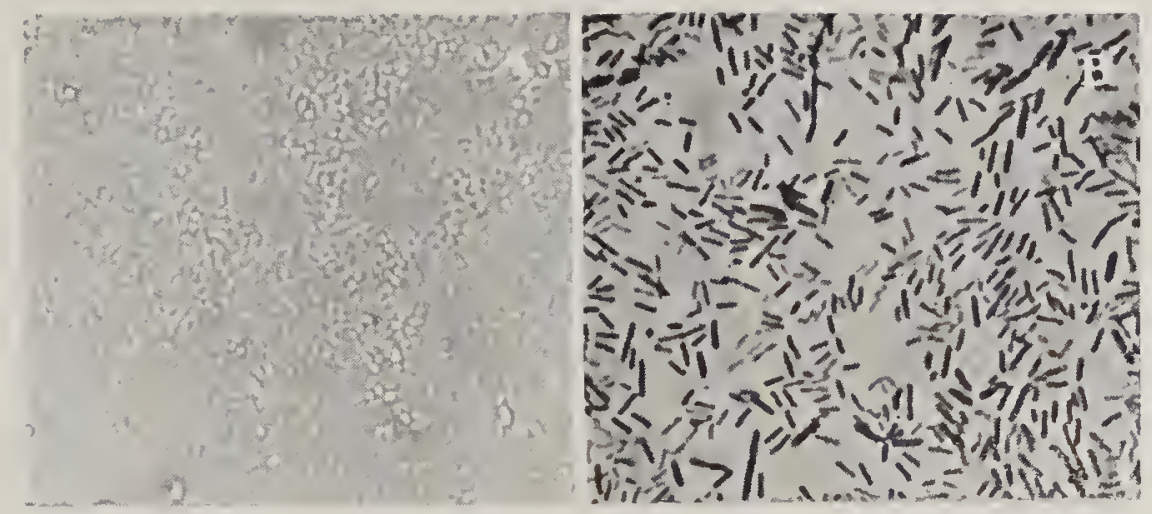

FI(i. I $(\times 500)$

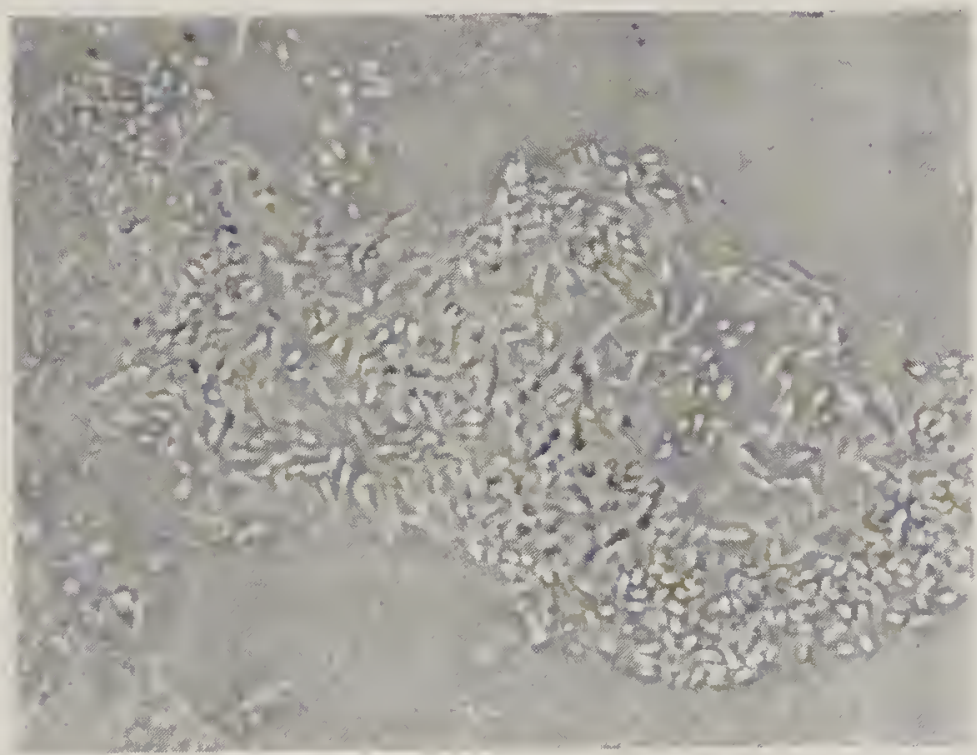

Filg. $2(\times 700)$

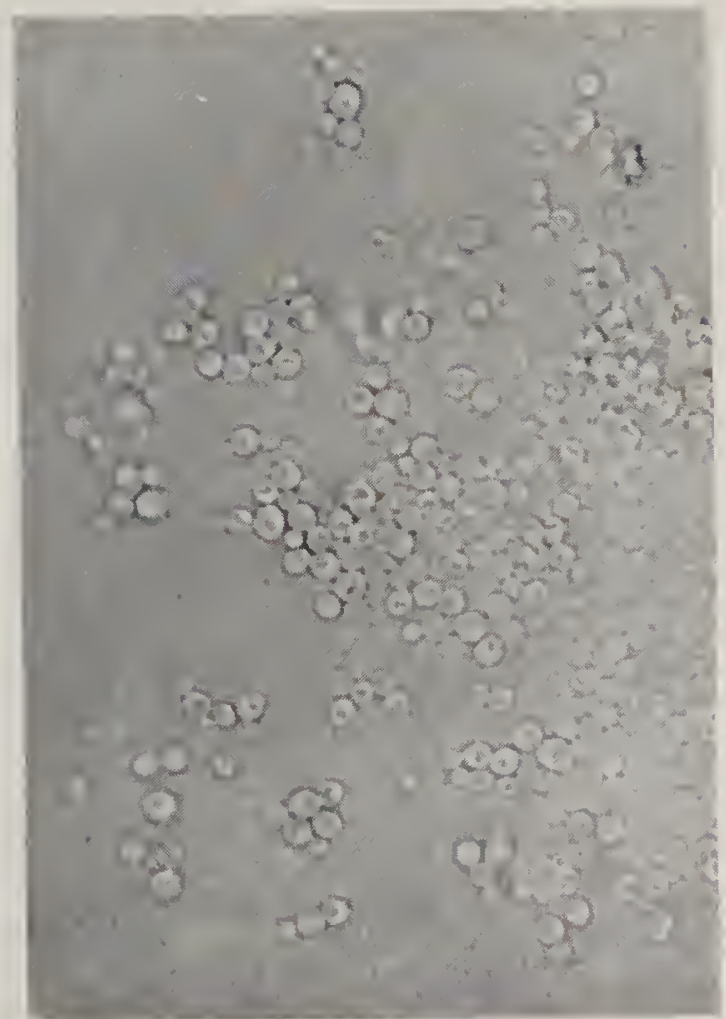

Fil. $3(\times 500)$

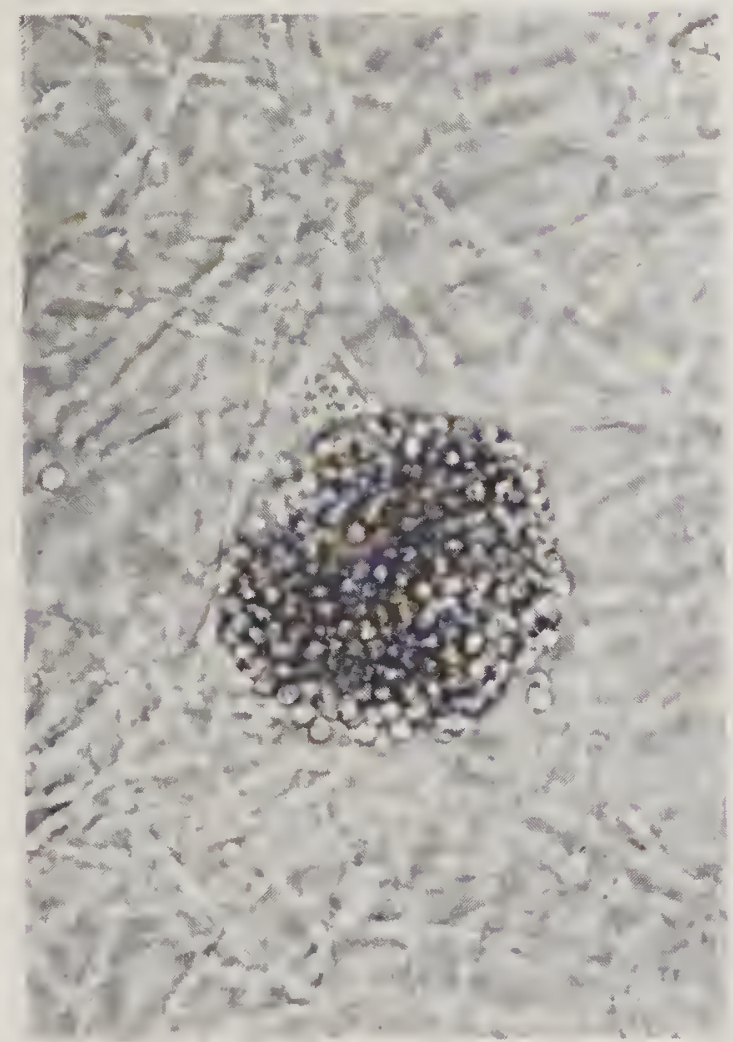

Fif $+4(x, 500)$

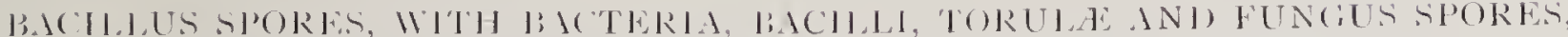

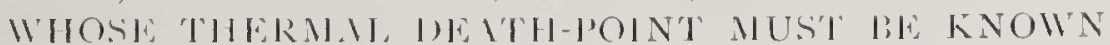





\section{(a) Death-point of Spores of Bacilli in the Moist State}

In 1876 I began the investigation of this problem with Bacillus spores obtained from urine, such organisms being almost exactly similar to those that are to be found in a hay infusion. They were procured in this way. I inoculated a neutralised specimen of boiled urine contained in a flask plugged with cotton wool, with another specimen of urine already swarming with Bacilli, and placed the mixture in an incubator at $100^{\circ} \mathrm{F}$.

In the course of two or three days a scum formed, in the threads of which spores were abundantly developed, and the filaments themselves, during these and the two or three subsequent days, broke up very extensively. This liquid (A), thoroughly well shaken, gave me a fluid, as I ascertained by examination, teeming with spores of Bacilli. Another liquid (B) was also prepared by causing neutral urine to ferment at $122^{\circ} \mathrm{F}$. in an airless vessel. In this fluid, while the Bacilli themselves were swarming in the form of rods or short filaments, their germs or spores were absent.

A number of bulb-tubes were then taken, and each of them was charged with one ounce of a urine whose acidity was equivalent to I 2 - I 5 minims of liquor potassæ per ounce. Such a fluid is one which can be certainly sterilised by raising it for a few minutes to $22^{\circ} \mathrm{F}$., and this, of course, is an essential property for any nourishing liquid that is 
to be used in experiments as to the death-point of organisms-we must know for certain that, under the conditions employed, the fluid is not a "generating" medium.

To some of the tubes charged with this fluid one minim of the fluid $A$, containing an abundance of spores, was added; while others were inoculated with a drop of fluid $B$, containing rods and short filaments but no spores.

The necks of the bulb-tubes, thus charged, were then drawn out in the blow-pipe flame, and the fluid within each was boiled over the flame for about a minute. The neck of the tube was hermetically sealed during ebullition, after which it was at once plunged in an inverted position into a can of boiling water where it was allowed to remain exactly ten minutes. ${ }^{1}$

The fluids of the two series, similarly heated, were then placed side by side in an incubator maintained at $122^{\circ} \mathrm{F}$. (50 C.), and the result in 25 trials ( 19 containing fluid $A$, and 6 containing fluid B) was that not one of either series fermented, although the tubes were kept from ten to fourteen days in the incubator. Yet, in control experiments with the same urine boiled for ten minutes in plugged flasks, and subsequently inoculated with an unheated drop of fluid A or of fluid B, fully developed fermentation was set up, with the appearance of swarms of Bacilli, in from sixteen to twenty hours: showing clearly that there was nothing in the

1 The object of inverting the vessel in the can was to bring all parts of the tube into contact with the heated fluid contained therein. 
nature of the fluid to impede the development of the organisms.

Having ascertained that hay-bacilli also grew and multiplied with about equal readiness in such acid urine, I subsequently executed a third series of experiments, in which the inoculating material was similar to that of $\mathrm{A}$ in the fact that it swarmed with Bacillus spores, only it was composed of hay-infusion instead of urine, in which the organisms had gone on to spore-formation. The results were, however, in no way different. Out of twenty-four trials with this fluid as the inoculating medium, fermentation did not take place in a single instance, the inoculated fluids having been boiled as before for ten minutes.

It seems perfectly clear, therefore, that the spores of Bacilli when in the moist state are all killed by an exposure to $2 \mathrm{I} 2^{\circ} \mathrm{F}$. ( $100^{\circ} \mathrm{C}$.) for ten minutes. Probably they would have been killed at some point intermediate between this and $158^{\circ} \mathrm{F}$.-the latter being the temperature which, as we have seen, kills all kinds of Bacteria except a very few of the thermophilic forms. What the exact death-point of these spores is, however, I did not ascertain at the time; and, so far as I know, it has never since been done.

In reference to the spores of Fungi, also, de Bary ${ }^{1}$ mentions that in their dry state $130^{\circ} \mathrm{C}$. has been necessary for their destruction, though he adds, "the death-point of the spores of Fungi is often much lower than this in water or watery vapour, and it has not been shown that any can

1 "The Structure and Functions of Bacteria," Transln., 1900, p. 76. 
under these circumstances survive a temperature of ${ }^{100^{\circ}}$ C. ${ }^{1}$ There is, in short, the concurrent testimony of many observers to the fact that, after such an exposure, germination would never take place because the spores were no longer living. This was the result obtained in many experiments made by Bulliard, and related in his "Histoire des Champignons." Mere contact with boiling water was found sufficient to prevent germination; and M. Hoffmann ${ }^{2}$ similarly ascertained that an exposure of from four to ten seconds sufficed to prevent the germination of all the Fungus spores with which he experimented. The experience of other observers has been similar, and among them that of Pasteur himself, who says explicitly, no Mould or Torula "can resist $\mathrm{IOO}^{\circ} \mathrm{C}$. when immersed in water," as he has satisfied himself-this time, by "direct experiments." 3 Professor Jeffries Wyman also said," "We have tried many experiments upon different kinds of Moulds and yeast plants, and have found, as nearly all observers have, that they perish at 2 I $2^{\circ}$ F." The experiments of Liebig and Tarnowski have, in fact, shown as already stated that

1 The difference of the resistance of organisms to dry heat is always far greater than to moist heat. But in all experiments referred to in this work we shall have to do with moist heat. I, therefore, purposely make no reference here to the investigations of Drs. Dallinger and Drysdale on the death-point of Monad germs (Monthly Microscop. Joum., Aug. I873, p. 57), though I have analysed and criticised these observations elsewhere (Joumal of Limn. Soc. [Zoology], vol. xiv. pp. 76-78).

2 Bullet. de la Soc. Botan., t. viii., p. So3.

3 Ann. de Chim. ct de Physique, i S62, p. 60.

4 "Observations and Experiments on Living Organisms in Heated Water," American Journ. of Science and Arts, vol. xliv. Sept. I867. 
even a temperature of $55^{\circ}-60^{\circ} \mathrm{C}$. is fatal to Torula and Fungus spores (see pp. 62 and 65 ).

\section{(b) Death-point of Spores of Bacilli after they have undergone Desiccation for a brief period}

This is a subject concerning which the most diverse statements have been made. Very great uncertainty exists even in regard to the ability to withstand desiccation possessed by the ordinary vegetative forms of Bacteria. One of the first to make observations on this latter subject was Burdon Sanderson, ${ }^{1}$ who definitely came to the conclusion not only that "the germinal particles of microzymes are rendered inactive by thorough drying without the application of heat," but also that "fully-formed Bacteria are deprived of their power of further development by thorough desiccation." The amount of desiccation adopted by Burdon Sanderson was merely that occasioned by keeping the organisms for two or three days in an uncovered condition exposed to a temperature of $104^{\circ} \mathrm{F}$. Subsequent investigations, however, have shown that his conclusion by no means holds good for all Bacteria, and that whether they survive or not depends much upon the conditions under which they undergo desiccation. His conclusion, also, most certainly does not apply to the spores of Bacilli, which were unknown at the time his observations were made.

It remained, therefore, to ascertain by exact experiments what evidence could be obtained, in support

1 Thirteenth Report of the Medical Officer of the Privy Council, I $87 \mathrm{I}, \mathrm{p} .6 \mathrm{I}$. 
or otherwise, of the statements which had been made to the effect that a previous desiccation enabled spores, in such a state, and when surrounded by their albuminoid or gelatinous envelopes, to resist for a long time the moistening influence of water, and thereby to withstand for long periods a degree of heat which would otherwise have proved destructive.

To test this point I proceeded in the following manner. I took a hay infusion on which there was a well-formed scum containing myriads of the most typical spore-bearing filaments, partly entire and partly breaking up, so as to liberate the spores. This was put into a corked test-tube and shaken vigorously for a short time, so as to procure a uniform dissemination of the spores through the liquid. Some of the thick, muddy-looking emulsion was then poured upon an ordinary clean microscope slip, so as to cover it with a thin stratum of the fluid, which was subsequently allowed to evaporate. In the course of three or four hours, when a dry opaque layer had been left upon the glass, the slip was placed in the dry chamber of an incubator at a temperature of $122^{\circ} \mathrm{F}$., where it was kept exactly four days. The dry layer was then scraped off with a knife into a clean watch-glass, and to the resulting powdery material about thirty or forty minims of distilled water were added.

After allowing the powder to remain thus immersed for four hours, so as to imitate the stage of preparation of a hay infusion, some of the stirred-up mixture was added to a quantity of urine having an acidity equivalent to eleven minims of liquor potassæ 
per ounce. The addition was made in the proportion of two minims of the spore-containing liquid to each ounce of the urine; and with this wellshaken mixture nine bulb-tubes were charged After their necks had been drawn out in the blowpipe flame, the fluid in each of them was boiled over the flame for rather less than one minute, when the vessel was hermetically sealed. An interval of nearly a minute having been allowed to elapse (so as not to crack the heated tip), each closed vessel was inverted and plunged into a can of boiling water, where it was allowed to remain for twenty minutes.

Subsequently all the tubes were placed together in an incubator at $122^{\circ} \mathrm{F}$, and with them a control experiment, consisting of a small flask plugged with cotton wool, in which some of the same urine had been boiled alone for twenty minutes, and to which two drops of the original spore-containing mixture (not previously dried or heated), were added when the urine had cooled. This latter operation was effected by removing the cotton-wool plug for an instant, allowing the spore-containing fluid to drop into the urine, and then carefully replacing the plug, after the manner so often adopted by Professor (now Lord) Lister. ${ }^{1}$

The results of these experiments were as follows: In sixteen hours the fluid in the control-tube was notably turbid, and at the expiration of twenty-four hours a thin scum of Bacilli had formed on its surface. The fluids in the other nine tubes all remained quite

${ }^{1}$ Quart. Jouml. of Microsc. Science, 1873, p. 384. 
clear, and showed no signs of turbidity during the ten days that they were retained in the incubator.

Thus thousands of Bacilli spores, after they had undergone a very considerable amount of desiccation, were found to be unable to survive a heating to 2 I $2^{\circ} \mathrm{F}$., prolonged for only twenty minutes.

\section{(c) Death Point of Spores of Bacilli after Desiccation for a prolonged period}

About the same time that these experiments were made (towards the middle of 1877 ) Professor Tyndall had much to say concerning "old hay germs," as a consequence of his having made experiments with some five-year-old hay, which yielded results he could only explain by imagining that certain Bacillus germs, not seen and examined by him, but supposed to be contained in the hay, had, by reason of their prolonged desiccation, been able to withstand the temperature of boiling water even for seven or eight hours, and thus to have been the cause of the appearance of swarms of Bacilli within his experimental vessels.

Recognising, at this time, the futility of further discussion on such a point till exact experiments bearing upon the question had been made of a kind similar to those just recorded-except for the fact that the Bacillus spores had actually undergone desiccation for a number of years rather than daysI caused another hay infusion to swarm with such bodies, and subsequently poured some of this fluid out upon six microscope slips, so as to form a thin stratum on each. After the fluid had evaporated, 
and a dry layer swarming with spores of the Bacilli was left, the slips were put away in a cabinet, protected from dust, and there left for nearly six years.

Then, in I883, the dry layer was at different times scraped off from the glass slips, the fragments were put into a purified test-tube, with about one drachm of distilled water, and were well shaken, so as to break up and distribute the particles and form a kind of emulsion. Here then we had an inoculating material, undoubtedly full of "old hay germs" which had undergone a prolonged desiccation, and were, consequently, in the condition deemed most favourablefor enabling them to escape the destructive influence of heat.

Two sets of experiments were made with this mixture; one in which this inoculating material was allowed to soak in the distilled water for four hours at a temperature of I I $2^{\circ} \mathrm{F}$., so as to imitate as much as possible what would happen in the preparation of an infusion; and another in which there was no preliminary soaking of the inoculating material for more than a very short time prior to the process of heating to which it was submitted. Sterilised acid urine was again used as the nourishing fluid in each set of experiments.

(I) Experiments with the Spore Liquid previously maintained at I I $2^{\circ} F$. for four hours. - At the expiration of the four hours, the spore liquid was added to the sterilised urine contained in a sterilised receiver, as before, in the proportion of two minims to the ounce. 
Twelve two-ounce retorts were then charged with the inoculated fluid, and the necks of the retorts were drawn out in the blow-pipe flame. Each fluid was boiled about one minute over a flame and sealed during ebullition; after which the retorts were inverted and plunged into a can of boiling water, for different periods :-

2 were boiled in the can for 5 minutes.

$\begin{array}{lllll}2 & \text {, } & , & \text { I0 } & , \\ 2 & ,, & , & \text { I } 5 & , \\ 2 & ,, & , & 20 & , \\ 2 & , & , & 25 & , \\ 2 & , & , & 30 & ,\end{array}$

The twelve retorts, after being heated, were placed in the incubator at $\mathrm{I}_{2} 0^{\circ} \mathrm{F}$., and the results were as follows:- The fluid in one of the retorts which had been heated for $3 \mathrm{O}^{\prime}$ to $22^{\circ} \mathrm{F}$., became turbid in two days, and was found to swarm with Bacilli ; while those in the other I r retorts remained quite clear at the expiration of I 4 days.

The scum containing Bacillus spores was scraped from another slip, and after soaking in a test-tube containing a drachm of distilled water for four hours at 2 I $2^{\circ} \mathrm{F}$, as before, it was dealt with in a rather different manner.

This spore liquid was added, in the same proportion, to a stock of sterilised urine contained in a purified Lister's receiver. Meanwhile a number of two-ounce sterilised cork flasks had been prepared, which, with the aid of an assistant, were charged rapidly, and in the most careful manner, after the 
fluid had been boiled for different periods. After charging, the flasks were quickly recorked, and placed in the incubator at $\mathrm{I}_{2} \mathrm{O}^{\circ} \mathrm{F}$. This trial differed from the other, therefore, in the fact that the flasks contained air. It led to the following results :-

Results.

3 flasks charged after fluid boiled $5^{\prime}\left\{\begin{array}{l}2 \text { turbid in } 24 \text { hours. } \\ \text { I clear after } 5 \text { days. }\end{array}\right.$

$3 \quad, \quad " \quad, \quad, \quad$ I $\mathcal{O}^{\prime}\left\{\begin{array}{l}2 \text { turbid in } 24 \text { hours. } \\ \mathrm{I} \text { clear after } 5 \text { days. }\end{array}\right.$

I8 other flasks were charged with this fluid, which had been boiled Io'; and then the fluid in each flask was boiled for $5^{\prime}$ more, making a total boiling for each

All the I 8 fluids quite clear after 5 days. $^{1}$ of 15 '.

Thus, in these 36 experiments only 4 flasks showed any evidence that the Bacilli spores, after prolonged desiccation, were able to survive a boiling of IO', and only I that they might be able to resist boiling for $3 \mathrm{O}^{\prime}$.

(2) Experiments with Spore Liquid heated without such a prolonged preliminary soaking.-Six twoounce retorts were charged, more than half full with sterilised urine, inoculated as before, and in the same proportion, with the scrapings, swarming with Bacillus spores, from another glass slip. The necks of the vessels were drawn out, as before,

1 It was found useless to keep these inoculated fluids under observation for a longer period. Growth and multiplication occurred within three days, if at all. 
and the fluid in each was boiled for about one minute, when the neck of the retort was sealed during ebullition. Subsequently the retorts were inverted, and immersed in a can of boiling water for different periods.

Two were boiled in the can for Io', within half an hour after the wetting of the spore material.

Two were boiled for $20^{\prime}$, within one hour after the wetting of the spore material.

Two others were boiled for $30^{\prime}$, within one and a half hours after the wetting of the spore material.

One of the retorts heated for $20^{\prime}$ was accidentally broken, but the other five were placed in the incubator at $120^{\circ} \mathrm{F}$, and there left for five days, all the fluids having remained clear and unchanged.

Another set of experiments was made with a slight variation in method. To a stock of sterilised urine in a Lister's receiver, a quantity of the spore liquid (after only a brief preliminary soaking) was added in the proportion of two minims to the ounce as before. From the inoculated fluid in the receiver, 23 small flasks plugged with carbolised cotton-wool were charged, and the fluid in each of the plugged flasks was gently boiled over the flame for I $2^{\prime}$. One of these flasks was accidentally broken. A control experiment was also prepared, in which the urine was boiled alone, and when it had become somewhat cool it was inoculated with two minims of the unheated spore liquid. All were then placed in the incubator at $120^{\circ} \mathrm{F}$., with the following results:-

The fluid in the control flask was found in 24 
hours to be turbid with Bacilli. At the expiration of 48 hours all the other 22 fluids were quite clear, but by the end of the third day two of them had become turbid, while the remaining 20 underwent no change.

Here again, then, out of 27 trials with a favourable nourishing fluid inoculated with desiccated Bacilli spores, under conditions most favourable for their survival, ${ }^{1}$ in only two cases where they had been heated merely for $\mathrm{I}^{\prime}{ }^{\prime}$ were they able to survive an exposure to $2 \mathrm{I} 2^{\circ} \mathrm{F}$.; while out of the total 63 trials in which these "old hay germs" (that 1s, actual spores desiccated for over five years) were used as the inoculating material, only once was there evidence that any of them could resist an exposure for $20^{\prime}$ to a temperature of $212^{\circ} \mathrm{F}$. - and it is quite possible that this isolated positive result might have been due to some accidental contamination after the heating.

With the last microscope slip containing these desiccated Bacillus spores an attempt was made to see what the influence of $80^{\circ} \mathrm{C}$. ( $\left(76^{\circ} \mathrm{F}\right.$.) upon them would be. Some acid urine was first sterilised by boiling it for $\mathrm{IO}^{\prime}$ in a purified Lister's receiver. To this some of the emulsion containing Bacillus spores, without any prolonged soaking, was added in the proportion of two minims to the ounce, as before.

1 Most favourable because in their dried state, just like a parched pea, they might be able to withstand for a time the influence of the boiling water, and subsequently undergo development while in the incubator. 
The inoculated mixture was then heated on a sand bath till a thermometer in the fluid (passed through the cock of the receiver) stood at $80^{\circ} \mathrm{C}$. $\left(\mathrm{I} 76^{\circ} \mathrm{F}\right.$.), and at this temperature it was maintained for $5^{\prime}$ only.

With this heated inoculated nourishing fluid I 2 purified flasks, plugged with carbolised cotton-wool, were most carefully charged, with all precautions. The plugs being replaced, each flask was put into the incubator at $\mathrm{I}_{20} \mathrm{~F}$., and there allowed to remain at this temperature. The results were as follows :-

One of the flasks was accidentally broken; but of the remaining I I all but three were lighter coloured and distinctly turbid from multiplication of Bacilli in 24 hours; by 36 hours two of the others were turbid; but up to the end of the fifth day the fluid in the one remaining flask was still quite clear. Thus out of I I trials, on ten occasions it was found that these desiccated Bacillus spores were able to resist a temperature from $10^{\circ}-20^{\circ} \mathrm{F}$. higher than the organisms from which they had been derived. What their precise death-point would be still remains rather uncertain, though it seems clear from the other $\sigma_{3}$ trials that there is a very remote chance for any of them being able to resist the destructive influence of $212^{\circ} \mathrm{F}$. for $20^{\prime}$. This conclusion will be found to be one of considerable importance in reference to the interpretation of other experiments to be recorded in this volume.

The only other point concerning the question of 
vital resistance to heat that remains for brief consideration is as to the death-point of the spores of the thermophilic Bacteria, which seem to be the most resistant of all. This question has been investigated by Christen, ${ }^{1}$ who arrived at the conclusion that the spores of these organisms, commonly found in soil, were killed by compressed steam in the following periods, when exposed to successively higher temperatures :-

\begin{tabular}{|c|c|c|}
\hline At $100^{\circ} \mathrm{C}$. in & & I 6 hours. \\
\hline,$\quad 105^{\circ}-110^{\circ}$ & "' & 2 to 4 hours. \\
\hline " I I $5^{\circ}$ & ", & 30 to 60 minutes. \\
\hline I $25^{\circ}-130^{\circ}$ & "' & 5 minutes and longer. \\
\hline $135^{\circ}$ & ", & minutes. \\
\hline $140^{\circ}$ & & I minute. \\
\hline
\end{tabular}

According to Lehmann and Neumann ("Principles of Bacteriology," 1906, p. 53), from whose work I have taken these figures, "the apparatus employed brought the objects to the desired elevation of temperature very quickly."

These are certainly very remarkable results when compared with the very much lower resistance to heat shown even by desiccated hay Bacillus germs. But, as I have intimated already, the thermophilic soil Bacteria are little likely to complicate our results. The fact that they are not to be found in tap water, and still less in distilled water, enables us practically to disregard them as possible contaminations in our experiments. We have only to take note of the fact that they are perhaps the very lowest forms of

${ }^{1}$ Centralblatt fïr Batiteriologie, xvii., p. 498. 
life, and possibly, in consequence thereof, are capable of resisting degrees of heat far higher than those which prove fatal to ordinary bacteria, including Bacilli, Micrococci, Streptococci, and Staphylococci. These latter, as we have seen in their vegetative forms, are, like Torulæ and Fungus-germs, killed by a brief exposure to $140^{\circ} \mathrm{F}$. $\left(60^{\circ} \mathrm{C}\right.$.), or thereabouts; while the more resistant, though much less widely distributed, spores of ordinary Bacilli have been shown to be killed, with only one exception, out of very numerous trials, when immersed in fluids that have been raised to the boiling point for $2 \mathrm{O}^{\prime}$.

After this exhaustive enquiry as to the degree of heat to which it is needful to expose our closed experimental vessels and their fluid contents, in order to feel reasonably sure that all pre-existing living things within them have been killed, we are in a position to take up the further enquiry, to which this has been a necessary preliminary, as to whether certain fluids, over and above their power of nourishing living things, are capable of actually engendering them. We have seen (p. 57) that some fluids are "nourishing" fluids only; we now have to ascertain whether some of these same fluids under other conditions, or other fluids altogether, may not, in addition, be "generating" fluids for different kinds of Bacteria, for Torulæ, and for some simple Moulds. 


\section{CHAPTER XI}

MODES OF TESTING THE QUESTION WHETHER CERTAIN SOLUTIONS CAN GIVE BIRTH TO SPECKS OF LIVING MATTER

NE of the best of the methods for investigating this problem was introduced so long ago as the middle of the eighteenth century, during the controversy that occurred between the learned Abbé Spallanzani, Professor of Philosophy at Modena, and Turberville Needham, a Catholic priest, who is said to be the first of the English Catholic clergy who had the honour of being elected a Fellow of the Royal Society. He was also a corresponding member of the French Academy, and was a friend of Buffon, the celebrated naturalist. They often worked together and shared one another's views, as may be seen from the early volumes of Buffon's great work on "Natural History," where an account of the views of Needham is to be found. In the controversy between him and Spallanzani, what is known as the "method of Spallanzani" was had recourse to in the most important of their experiments. ${ }^{1}$ It

${ }^{1}$ An account of these experiments may best be seen in a French translation of a work by Spallanzani entitled "Nouvelles Recherches sur les Découvertes Microscopiques," 1769, with copious notes by Needham. See especially pp. 8, 102-135, and 216-2 I8. 
was extremely simple and yet perfectly suitable. The infusions with which trial was to be made were introduced into flasks with narrow necks; the necks of the flasks were drawn out and then hermetically sealed in the blow-pipe flame, while the flasks with their contents were subsequently heated in boiling water for different periods-with the view of killing any pre-existing living things, either in the fluids, in the air contained within the flasks, or on the walls of the vessels themselves.

In ${ }^{8} 37$ another mode of experimentation was introduced by Schwann, ${ }^{1}$ and has been much used since his time (either exactly or with slight modi-

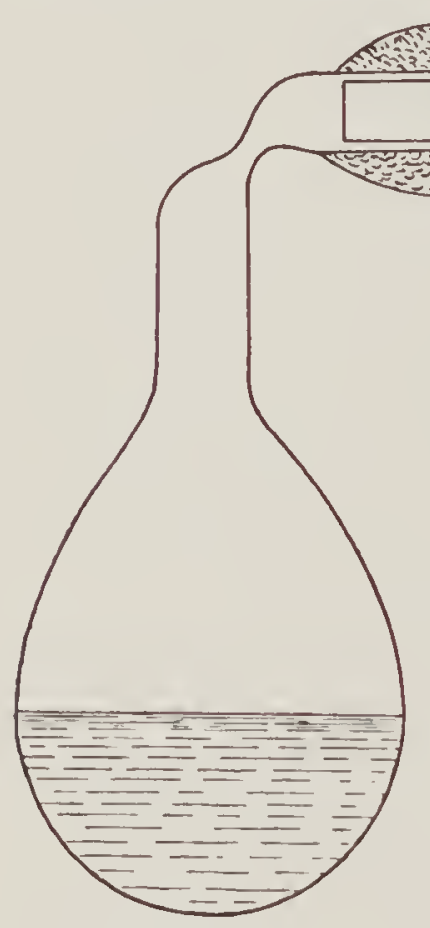

FIG. I. fications), and principally by Pasteur and Jeffries Wyman. The organic matter in solution with which trial is to be made is boiled in a flask, the neck of which is securely connected with a tube closely packed with portions of redhot pumice stone-or else closelypacked, fine, iron wires as employed by Wyman. And after the solution has been boiled for some time, so that all the air of the flask has been Wyman's Modifica- expelled, the vessel itself is allowed tion of Schwann's to cool slowly, while the tube con-
Apparatus. taining the closely-packed red-hot materials is still maintained at the same temperature, in order that the air entering slowly into ${ }^{1}$ Pogendorff's Amalen, 1837 , vol. xli. p. 184. 
the flask may be subjected to a calcining heat as it passes through the tube. When the flask has become cool, its neck is hermetically sealed by the blow-pipe flame, so that it will then contain only the previously boiled solution in contact with air (at ordinary atmospheric pressure) which has been calcined.

At other times Pasteur has boiled the organic solution over the flame, in flasks whose necks have been plugged with cotton wool; or else in flasks with long, narrow and bent necks. In the first case air would only enter the slowly-cooling flask after it has been filtered from its impurities by the cotton wool; while, in the second form of flask, the air which is allowed to re-enter slowly, during gradual cooling, is supposed to deposit its impurities in the flexures of the long and bent neck. Such methods cannot be as safe as the use of hermetically sealed vessels.

Although the presence of air within the closed flasks has generally been considered essential, still it had been shown by Fray, ${ }^{1}$ even before the time of Schwann, that atmospheric air might be replaced by other gases such as hydrogen or nitrogen; and that even then (with the method of closing the vessels at the time in vogue) living organisms were subsequently to be met with in the infusions. Later, Professor Mantegazza ${ }^{2}$ and Pouchet ${ }^{3}$ showed that oxygen gas might be successfully substituted for atmospheric air, in experiments which in other

1 "Essai sur l'origine des corps organisés et inorganisés," Paris, I 82 I, pp. 5-8.

"Giomale dell R. Instituto Lombardo, t. iii. I851.

${ }^{3}$ Compt. Rend. (1858), t. xlvii. 
respects complied with Schwann's conditions; while Dr Child ${ }^{1}$ also showed that organisms were to be met with when either oxygen or nitrogen was substituted for atmospheric air in similar experiments.

It was stated by Spallanzani that while organisms were procurable from hermetically sealed flasks in which the air was somewhat rarefied, they were not to be met with when the rarefaction was extreme, or where a vacuum existed. Pouchet also rejected, as preposterous, the notion that organisms could be expected to occur under such conditions ${ }^{2}$ and both he, as well as Pasteur, whenever desiring to make comparative trials with the air of different localities, boiled the fluids and sealed the necks of the flasks during ebullition-never supposing that any fermentative changes could occur in the fluids contained in these airless flasks. ${ }^{3}$

In the spring of I870, however, after a few tentative trials, I definitely adopted this mode of experimentation, and soon had reason to think that some infusions were rather more prone to undergo change in these retorts, from which almost all the air had been expelled, than when they were contained in flasks full of air, prepared after the manner of Needham and Spallanzani.

It occurred to me, at that time, that putrefactive or fermentative changes might not always be initiated

1 "Essays on Physiological Subjects," 2nd ed., I 869, p. I I 4.

2 "Nouvelles Experiences," I 884 , p. I 2, note, and p. I 56.

${ }^{3}$ Such flasks were carried away by them to the Alps or Pyrenees, in order that the necks of the flasks might be broken in the localities chosen, the influence of whose air they wished to test. After being resealed the flasks were kept for future observation of their contents. 
by contact of organic matter with oxygen or any other gas-that it might, at times, be dependent upon the inherent instability of the organic matter itself. Independently of the fact, therefore, that the sealing of the flask after all the air had been expelled, and during ebullition of the fluid, was a much simpler process than having to admit calcined air and sealing the flask after it had cooled, it seemed possible that the existence of a comparative vacuum might, for other reasons, sometimes prove an advantage.

The method, therefore, adopted by me for a long time was this. After each flask, of one to two ounces capacity, had been thoroughly cleaned with hot water, three-fourths of it was filled with the fluid that was to be made the subject of experiment. The neck of the flask or retort was then drawn out in the blow-pipe flame, two or three inches from the bulb, till it was about a line in diameter. The neck having been cut across in this situation, the fluid within the vessel was boiled continuously for from ten to twenty minutes. At first, ebullition was allowed to take place rapidly (till some of the fluid itself frothed over) so as to procure the more thorough expulsion of the air and bring all parts of the flask in contact with the boiling water; then the boiling was maintained for a time at medium violence over the flame of a spirit-lamp, and the neck of the flask was subsequently sealed in the blow-pipe flame during ebullition.

After a little practice one soon became able to 
seal the flasks actually during ebullition, that is, while there was still an out-pouring of steam; and it must be borne in mind that even if the outpouring of steam had ceased for a second before the almost capillary orifice of the heated neck of the flask had been closed, the neck of the flask being at the time in the blow-pipe flame, air could only enter through the flame and through the white hot capillary orifice itself. It would, in fact, have been calcined as in Schwann's experiment. The conditions of the experiment would thus have been no less severe, and the only effect would be that the vacuum would have been rendered a trifle less complete.

In many of my later experiments with this method a slight modification was made. The fluid was boiled for a shorter time over the flame, and as soon as the flask was hermetically sealed, it was inverted and placed in a can of boiling water for the remainder of the time during which it was to be heated. This modification was adopted with the view of meeting objections that had been made, and in order that all parts of the vessels employed should be more effectually brought into contact with boiling water.

There is only one other principal method-namely that employed by Professor Tyndall, and by no one else. It was a most complicated and uncertain mode of experimentation, which undoubtedly caused him much trouble, and led to most contradictory results. Some account of it will be given later on (Chapter xviii.), when reference will have to be made to these experiments. 
In my own experiments, dealing with the major issue, I have invariably used hermetically sealed flasks or tubes, and the experiments have either been carried out in the manner I have just described, or else by adopting the equally safe method of Needham and Spallanzani. 



\section{PART III}

THE EXPERIMENTAL EVIDENCE IN REFERENCE TO PASTEUR'S CONCLUSIONS

\section{CHAPTER XII}

WAS PASTEUR RIGHT IN SAYING THAT GUARDED ACID FLUIDS PREVIOUSLY HEATED TO IOO $^{\circ}$ C. ( 2 I $2^{\circ}$ F.) REMAIN BARREN?

I HAVE already, in Chapter v., pointed out the 1 enormous influence which Pasteur's memoir, published in 1862, has had in building up an exclusive belief in the germ-theory, and in casting doubt upon the present de novo origin of living matter. In one of the concluding pages of this chapter I have also set forth the fundamental inductions and corollaries upon which Pasteur's doctrines were based; and I propose now to examine these in detail, since they were strictly adhered to by him up to the date of his latest writings on the subject in 1877 , when he still showed himself very intolerant of any one doubting their truth.

He laid down the rule that fluids having an acid reaction would, after they had been boiled for a few minutes, and then efficiently guarded from contamination by particles contained in the atmosphere, remain 
pure for an indefinite time, and show no evidence of the existence therein of living things. These supposed facts confirmed him in the notion that all ferment organisms and their germs were speedily killed in such acid fluids when they were raised to the temperature of $100^{\circ} \mathrm{C}$.

The lethal influence of such a temperature was, in fact, at that time and subsequently, generally admitted, and was the cause of the great incredulity commonly felt in regard to the results of certain experiments recorded by me in $1870-72$; and was also the cause of the suggestions, made by Professor Huxley and others, that the organisms found by me in my experimental vessels were dead rather than living organisms.

Thus, at one of the Sectional Meetings of the British Association in I870, the President, after referring to some of my experiments, and to the fact that all unmistakably vital movements ceased after Bacteria had been heated to 2 I $2^{\circ} \mathrm{F}$., added : ${ }^{1}$ - "I cannot be certain about other persons, but I am of opinion that observers who have supposed that they have found bacteria surviving after boiling, have made the mistake which I should have done at one time, and, in fact, have confused Brownian movements with true living movements." His belief was so strong, on the one hand, that no Bacteria could withstand the temperature of boiling water, and on the other, as to the accuracy of Pasteur's conclusions, that he did not hesitate then, as on other occasions, to suggest that the organisms I had

${ }^{1}$ See Report in Quart. Journ. of Microsc. Scicncc, Oct. I87o. 
found in my experimental vessels were only dead organisms, previously contained in the fluids and killed by the heat.

My answer to this was given shortly afterwards in the following terms: ${ }^{1}$ - "If fluids in vacuo (in hermetically sealed flasks), which were clear at first, have gradually become turbid; and if, on microscopical examination, this turbidity is found to be almost wholly due to the presence of Bacteria or other organisms; then it would be sheer trifling gravely to discuss whether the organisms were living or dead, on the strength of the mere activity or languor of the movements which they may be seen to display. Can dead organisms multiply in a closed flask to such an extent as to make an originally clear fluid become quite turbid in the course of two or three days?"

The same utter incredulity, however, continued to be felt concerning the results of my experiments. This cannot be better shown than by reproducing some passages from a review of my then recently published work, "The Beginnings of Life," which appeared in the Academy of November I, I872, signed by H. M. Moseley, who subsequently distinguished himself by his investigations as one of the naturalists of the Challenger expedition. This writer says :-

"Dr Bastian seals the flasks with which he is experimenting during ebullition of the contained fluid, and by this means, when the apparatus has become cool, a partial vacuum is formed in the vessel. Experiments were made in this way with hay and turnip

1 "Modes of Origin of Lowest Organisms," I 87 I, p. 9. 
infusions, in which every possible precaution appears to have been taken to exclude or destroy germs. In nearly all cases, after the lapse of some time, the solutions became turbid, or exhibited a scum, and microscopic examination showed the existence of organic bodies in the fluids, and in some cases of bacteria in active motion."

"Now the only possible answer to be made to experiments such as these is that the turbidity or scum in the solutions was not caused by a development of organisms, but by some coagulation or similar alteration in the fluid, and that the bodies seen in the solutions were not living, but dead, and had been there all the time. ..."

"Considering, on the one hand, the $a$ priori improbability of the formation of bacteria, etc., de novo, with the great weight and high value of the evidence already adduced against its occurrence, and estimating, on the other hand, the value of the evidence here put forth, it seems very unlikely that Dr Bastian's results will be confirmed."

It would have been easy to add other expressions of opinion of a like kind from other eminent biologists, instigated doubtless under the influence of Huxley's suggestions and the glamour of Pasteur's authority; but enough has been said to show the feeling existing: and as I found that this feeling was evidently shared by my then colleague Professor (afterwards Sir) J. Burdon Sanderson, I suggested to him in December $\mathrm{I} 872$, that I should perform some of my experiments in his presence, on the understanding that he should subsequently publish an account of them, and of the results obtained. To this he assented, and the following report was published by him in Nature, January 8, i 873 . 

"Dr Bastian's Experiments on the Beginnings of
Life."

In every experimental science it is of great importance that the methods by which leading facts can be best demonstrated should be as clearly defined and as widely known as possible. This is particularly true as regards physiology, a science of which the experimental basis is as yet imperfect. All experiments by which a certainty can be shown to exist where there was before a doubt, serve as foundation stones. It is well worth taking some pains to lay them properly.

Your readers are aware that Dr Bastian, in his work on the Beginnings of Life, has asserted that in certain infusions the "lower organisms" come into existence under conditions which have been generally admitted to exclude the possibility of the pre-existence of living germs. It is also well known that these experimental results are disputed.

Not long ago I witnessed the opening of a number of experimental flasks charged many months ago by a friend of mine with infusions supposed to be similar to those recommended by Dr Bastian. The flasks had been boiled and closed hermetically according to Dr Bastian's method. Finding on careful microscopical examination that the contents of the flasks contained no living organisms, I charged calcined tubes with the liquids, sealed them hermetically, and forwarded them to Dr Bastian. When I next saw him he pointed out that two of the three liquids used were not those which he had 
recommended, that if the infusions had been properly prepared, there would not have been any necessity for keeping them many months before examination, that his results with organic infusions were obtained after a few days, and that they were generally of a most unmistakable nature. To satisfy my doubts on the subject he most kindly offered to repeat his experiments relating to the production of living organisms in infusions of hay and turnip in my presence. To this proposal (although I have hitherto taken no part in the controversy relating to spontaneous generation, and do not intend to take any) I gladly acceded, at the same time engaging to publish the results without delay.

Fifteen experiments were made. They were in three series, the dates of which were respectively, Dec. I 4, Dec. 20, and Dec. 27.

\section{First Series - (Dec. i 4th.)}

Two infusions were employed, an infusion of turnip, in making which both the rind and the central part were used, and an infusion of hay. Both had been prepared the same day a short time before they were used.

The turnip infusion, of which the specific gravity was IOI2, and the reaction distinctly acid, was divided into two parts, of which one was neutralised with liquor potassa. Four retorts, each capable of holding, when half full, a little over an ounce of liquid, having been prepared, two were charged 
with neutral infusion, the other two with unneutralised infusion. A small quantity of pounded cheese was then added to one of each pair. A fifth retort was charged with unneutralised infusion diluted with its bulk of water. As soon as each retort was charged, the open end of its beak was heated in the blow-pipe flame and drawn out. The drawn-out part was then severed, and the retort boiled over a Bunsen's burner, after which it was kept in a state of active ebullition for five minutes. During the boiling, some of the liquid was frequently ejected from the almost capillary orifice of the retort. At the end of the period named it was closed by the blow-pipe flame, care being taken to continue the ebullition to the last. The success of the operation was ascertained in each instance by observing that, by wetting the upper part of the retort, the ebullition was renewed.

Three similar retorts were charged with the hay infusion, the specific gravity of which was I005, and the reaction neutral. Of these, one contained the infusion diluted with its bulk of distilled water, the others being charged with infusion to which no addition had been made. These three retorts were closed, after boiling, in exactly the same way as those containing turnip infusion. The eight retorts were placed, immediately after their preparation, in a water-bath, which was kept at a temperature of about $30^{\circ} \mathrm{C}$.

We met to examine the flasks on December i 7 , just three days after their preparation, Dr Bastian having previously expressed his anticipation that the 
infusions of turnip with cheese, whether neutralised or not, would be found by that time to contain multitudes of Bacteria, and that the other two undiluted turnip infusions would exhibit obvious changes. In the hay infusions, he expected that the process would not advance so rapidly; the diluted infusions, he thought, would remain permanently unaltered. The results in each case were as follows :-

(a) Neutral turnip infusion with cheese.-On the I6th I observed that the liquid had become turbid; on the $\mathrm{i} 7$ th the turbidity was very obvious. Before opening the retort it was ascertained that when the blow-pipe flame was directed against the tube the heated part was drawn inwards, and further, that when the retort was inclined with its bulb upwards, so as to allow the liquid to rush against the closed end, a characteristic water hammer sound was produced. On breaking the point air rushed in with a tolerably loud sound; the liquid was crowded with moderately sized Bacteria, which exhibited active progressive movements. There were also Leptothrix filaments.

(b) Unneutralised tumip infusion with checse.On the I 7 th, the retort, having been tested in the same way as before with similar results, was opened. It contained no living forms.

(c) Neutral tumip infusion without chose.-On the I 7 th this liquid exhibited no marked change. It was finally examined on the 3 Ist, and found to be still unaltered.

(d) Unneutralised turnip infusion wihout cheese. 
- Up to December 31 no change had taken place in this infusion.

(e) Unailuted hay infusion.-The infusion was slightly turbid on the 17 th; on the 20 th the turbidity was more marked, and before the flask was opened, the water-hammer sound and other evidence showed that it was entire. The liquid was found to be full of minute but very active Bacteria, and contained numerous colonies of spheroids undergoing transformation into Bacteria. There were also Leptothrix filaments.

$(f)$ The same.--This infusion was examined on the same day. It had become turbid at about the same time as the last infusion, though to a less extent. It was distinctly acid. A drop of this fluid contained few Bacteria as compared with $e$.

$(g)$ Diluted hay infusion.-. On the 2oth it was discovered that the retort was accidentally cracked. The liquid was swarming with Bacteria, and possessed an offensive smell. On account of the crack, Dr Bastian regarded the experiment as futile.

(h) Diluted turnip infusion. - This liquid remained unchanged.

\section{Second Series-(Dec. 20th.)}

The purpose of this series was to ascertain whether the irregularities of the results with the turnip infusions in the first series, as compared with Dr Bastian's already recorded results, were due to the fact that the material used consisted partly of rind. Dr Bastian thought that this might be the 
case, and accordingly another infusion was prepared in which no rind was employed. As before, the fresh acid infusion of turnip was divided into two parts, one of which was neutralised by liquor potassæ. Of four retorts, three were charged with unneutralised liquid, the fourth with neutral. Of the three, two were treated with cheese; to the third no addition was made. They were prepared in every respect as before. In each case the drawing-in of the glass in the blow-pipe flame was again noticed before the neck of the retort was broken.

(a) Unneutralised infusion with cheese. - This infusion showed opalescence, even after twenty-four hours. On the $23 \mathrm{rd}$ it had become decidedly turbid, and was opened. The liquid was fœtid, and its reaction acid. It swarmed with Bacteria.

(b) The same.-The retort was opened on the 3 Ist, its contents having shown a slight turbidity for several days previously. The liquid was slightly foetid, and it contained characteristic Bacteria, which, however, were few in number.

(c) Nentral infusion without cheese.-The retort was opened on December $3 \mathrm{I}$, the fluid having been slightly turbid for several days. The liquid was acid, and slightly foetid, but still retained the odour of turnip. A drop contained a few Bacteria, about $0.003 \mathrm{~mm}$. in length, which exhibited oscillatory movements.

(d) Unnentralised infusion without cheese.-The liquid contained a white mass which lies at the bottom, and was so tenacious that it could be drawn out into strings with needles. This consisted entirely 
of Bacteria and Leptothrix, embedded in a hyaline matrix. There were also Bacteria in the liquid.

\section{ThIRd SERIES-(Dec. 27th.)}

It appeared to me desirable to ascertain whether the condition of the internal surface of the glass vessels exercised any influence on the result. I therefore heated two retorts to $250^{\circ} \mathrm{C}$., keeping them at that temperature for half an hour, and closed them while hot in the blow-pipe flame. These Dr Bastian charged by breaking off their points under the surface of a neutral infusion of turnip with cheese, freshly prepared for the purpose, without employing any of the rind. The retorts were boiled and sealed in the same way as before, excepting that whereas one was boiled only five minutes the other was boiled ten minutes. The specific gravity of the infusion used was IOI3. A third uncalcined retort was charged with some of the same infusion containing no cheese. This was also boiled for ten minutes.

I was out of town from the 28 th to the 30 th, and therefore did not examine the retorts until the 3 Ist. Dr Bastian informed me that on the 28th, twentyone hours after preparation, the liquids in both the calcined retorts were distinctly turbid, the temperature of the water bath being $32^{\circ} \mathrm{C}$. : and that sixty-six hours after preparation, whilst the turbidity was much more marked, each flask also contained what appeared to be a "pellicle," which had formed and sunk. At this period the fluid in the third flask had also become very decidedly turbid. 
(a) Neutral tumip infusion with cheese in calcincd retort, boiled ten minutes. - The retort having been tested in the way previously described was opened on the 3 ist. The liquid was very fotid, had an acid reaction, and contained much scum. It was found to be full of Bacteria, whilst Leptothrix existed in abundance in portions of the scum, together with granules of various sizes which refracted light strongly.

(b) The same boiled fio mimutes. - The state of the liquid was the same as that just described.

(c) Neutral infusion withont chess, boiled ten minutes-retort not calcinct. - In this liquid the rods and filaments were much less numerous. In other respects its characters were the same.

In each case before opening the retort it was again observed that a portion of its neck became drawn in when exposed to the blow-pipe flame.

As regards the results of the foregoing experiments, it is unnecessary for me to say anything as to their bearing on the question of heterogenesis. The subject has already been frequently discussed in your columns.

'The accuracy of Dr Bastian's statements of fact, with reference to the particular experiments now under consideration, has been publicly questioned. I myself doubted it, and expressed my doubts, if not publicly, at least in conversation. I am content to have established - at all events to my own satisfaction-that, by following Dr Bastian's directions, infusions can be prepared which are not deprived, 
by an ebullition of from five to ten minutes, of the faculty of undergoing those chemical changes which are characterised by the presence of swarms of Bacteria, and that the development of these organisms can proceed with the greatest activity in hermetically-sealed glass vessels, from which almost the whole of the air has been expelled by boiling.

\section{J. Burdon Sanderson}

University College, January 1.

Shortly after the publication of this report by Professor Burdon Sanderson there came another independent confirmation of my experiments in a letter published in Nature, March 20, 1873 , from $D$. Huizinga, the Professor of Physiology in the University of Groningen, from which some extracts may now be made. His communication was an important one, because it answered also some of the objections which had in the interval been raised by others, and pointed out the special necessity of care, if such experiments were to be attended by successful results.

Professor Huizinga wrote as follows: "Having occupied myself for some time past with an experimental study of abiogenesis, I have followed with much interest the controversies on this question in recent numbers of Nature, and beg leave therefore to state to the readers of this journal the results of my experiments. A turnip decoction of the specific gravity I०II-I०I6, filtered and boiled with cheese $\left(0^{\circ} 25^{-0} 5\right.$ grm. to 50 c.c.) ; filtered again and 
neutralised; then boiled for ten minutes and hermetically sealed, is, after two to three days' exposure to a temperature of $30^{\circ} \mathrm{C}$., swarming with Bacterium terms. It is, however, to be remarked that a too great concentration of the solution hinders the evolution of the Bacteria; the volume of the liquid employed should therefore not be too small, otherwise the boiling for ten minutes will render the solution too much concentrated. Perhaps the negative results recorded by some experimenters are to be explained in this way." . . .

"Instead of cheese can be used peptone (0.2 grm. to 50 c.c.) with the same result. The peptone is obtained by digestion of egg albumen with artificial gastric juice, subsequently isolated and purified by repeated precipitation with alcohol."

Huizinga then went on to describe the constitution of a liquid prepared from certain saline substances, together with grape sugar, which could be used in place of the turnip infusion, and which, with the addition of peptone, also yielded swarms of Bacteria when it was boiled and subsequently guarded from contamination.

Burdon Sanderson, however, in some remarks subsequently made (Nature, October 2, is73) concerning Huizinga's experiments, said: "The substitution of a soluble immediate principle for an insoluble mixed product like cheese, and the use of a definite solution of sugar and salts are not material improvements. The question is not whether the germinal matter of Bacteria is present, but whether it is destroyed by the process of heating. Con- 
sequently, what is necessary is not to alter the liquid, but to make the conditions of the experiment as regards temperature as exact as possible. In this respect Huizinga's experiment is a confirmation of Bastian's and nothing more."

These remarks are perfectly just. From the point of view of the interpretation to be given to the experiments it was immaterial how many germs were in the materials used, the real question was, as Burdon Sanderson said, whether germs that were present would be destroyed by the process of heating employed.

For the present, moreover, we are not considering the question of interpretation-we are concerned solely with a question of fact, namely, was Pasteur right or wrong in proclaiming that fluids having an acid reaction, after being boiled for a few minutes in glass vessels and subsequently guarded from atmospheric contamination, would subsequently show no signs of fermentation and reveal no living things therein? As regards this question of fact my experiments have shown that he was wrong. In addition to acid turnip infusions, such as were used in some of my experiments with Burdon Sanderson, I had obtained similar results with other acid fluids; and I shall have to record many new successful results in Chapters xix. and xx., with acid solutions containing an ammoniacal and other salts.

The columns of Nature during i 873 contain much discussion concerning these old experiments to which we have been referring; but as the letters and articles there to be found had almost solely to do with the 
question of interpretation, it is unnecessary to speak of them now-especially as most of the points referred to will have to be dealt with in subsequent chapters, and the question of interpretation must be delayed till the evidence as a whole has been produced.

There is one point, however, to which it is worth while to refer now. The amount of powdered cheese contained in any one of my flasks was certainly not more than two grains, and none of the particles was bigger than a pin's head, yet some critics talked of the protective influence of "lumps," and seemed to think that some Bacteria contained within such small particles of cheese could thereby escape the destructive influence of heat. This was the view brought forward after it had become perfectly certain that swarms of living Bacteria did, in fact, appear within my experimental vessels.

But this was a complete change of front on the part of the critics. For in 1870 , when Professor Huxley, and others who adopted his view, disbelieved in the presence of living Bacteria in my experimental vessels, they were willing to believe that comparatively large masses of solid and semi-solid matter could be effectively sterilised by a temperature of $212^{\circ} \mathrm{F}$. Thus Professor Huxley, in his presidential address after referring to such experiments as mine (but without mentioning names), made the following plausible, but very misleading, statements: "The first reply that suggests itself is the probability that there must be some error about these experiments, because they are performed on an enormous scale 
every day with quite contrary results. Meat, fruits, vegetables, the very materials of the most fermentable and putrescible infusions, are preserved to the extent, I suppose I may say, of thousands of tons every year by a method which is a mere application of Spallanzani's experiment. The matters to be preserved are well boiled in a tin case provided with a small hole, and this hole is soldered up when all the air in the case has been replaced by steam. By this method they may be kept for years, without putrefying, fermenting, or getting mouldy."

A few days after this statement was made I visited one of the largest establishments in London in which meats and vegetables are preserved. For this opportunity, and for many particulars communicated in a long conversation, with permission to make the facts known, I was indebted to the courtesy of $\mathrm{Mr} \mathrm{M}$ 'Call of Houndsditch.

A number of cases, enclosing the provisions, instead of being simply heated to a temperature of 2 I $2^{\circ} \mathrm{F}$, as most people would understand from what Professor Huxley said, are first heated in a large chloride of calcium bath (warmed by steam) and raised to a temperature of $230^{\circ}$ to $235^{\circ} \mathrm{F}$. for more than an hour and a half. The hole through which the steam has been issuing is then closed with solder. Of course the more or less solid contents of the tins would require a longer time to be raised to any given temperature than a fluid, but the probability is that during the latter half of this time the temperature of the contents would be not far short of $225^{\circ} \mathrm{F}$., at least. 
I have ascertained that boiling a quantity of fluid briskly in a vessel with a very narrow aperture very distinctly raises the boiling point. ${ }^{1}$ This I discovered by introducing a small maximum registering thermometer into one of the experimental retorts containing an ounce of a hay infusion, drawing out its neck so as to leave only the usual narrow orifice, and then boiling the fluid briskly for five minutes. The thermometer was an accurate one made specially for me by Hicks of Hatton Garden; its bulb was kept about three quarters of an inch away from the glass, and yet at the end of five minutes it was found to register $103.33^{\circ} \mathrm{C}$. (2 I $8^{\circ} \mathrm{F}$.). With a larger quantity of fluid, and the same small orifice, especially when the heating was continued over a prolonged period, as in the preparation of the tinned meats and vegetables, the temperature of the contents may well have been raised to about $225^{\circ} \mathrm{F}$. ( $107 \frac{1}{2}^{\circ} \mathrm{C}$. ).

Much would depend upon the smallness of the aperture together with the bulk of the fluid, as was shown by a letter in the same number of Nature (July IO, I873) from W. N. Hartley, who said :-

"I sealed a tube on to a flask of about roo c.c. capacity at right angles to the neck, and drew out the end so as to form a capillary orifice. About 30

${ }^{1}$ See Nature, July 10, I873. A similar elevation of temperature, to a certain extent, would occur in experiments conducted after the manner of Schwann; or when flasks with long, narrow and bent necks, or others whose necks were plugged with cotton wool, were employed, as was done by Pasteur. The temperature in all these cases would certainly be raised to a point over $212^{\circ} \mathrm{F}$. 
c.c. of water were put into the flask and a thermometer in an india-rubber cork was wired into the neck. On boiling the water the steam had not issued more than half a minute, before the temperature was $102^{\circ} \mathrm{C}$, and in less than ten minutes it had reached I $8^{\circ} \mathrm{C}$.; fearing the safety of the apparatus I did not proceed further, nor indeed did I wish to do more . . . that an aqueous solution may so easily be raised to 1 I $8^{\circ} \mathrm{C}$. is a point in chemical manipulation which will be turned to advantage in the laboratory."

It will be seen that the temperatures obtained by $W$. N. Hartley were much higher than those I have recorded, the difference probably depending upon the fact that the narrow orifices of my retorts (which were about one line in diameter) were not so small as that in his experimental vessel.

But in the preservation of the meats, immediately after the soldering, when the temperature of the conterits of the tin has probably been raised for some time to at least $225^{\circ} \mathrm{F}$., a higher pressure of steam is turned on by which the bath is quickly raised to a temperature of $25^{\circ}$ to $260^{\circ} \mathrm{F}$.- -at which temperature it is maintained for more than half-an-hour. The tins being now firmly closed the temperature of their contents would be gradually raised. While I was in this establishment one of the baths was seen to have reached a temperature of $263^{\circ} \mathrm{F}$. Thus it would seem that the contents of the tins are often raised for a short time as a final process to $260^{\circ} \mathrm{F}$. ( $126 \frac{1}{2}^{\circ}$ C.). 
All this is very different from the simple statement that the provisions are or were "boiled," for a time not specified. Professor Huxley, in the next place, mentioned the possibility of failures, though he seemed to attribute all these to "unskilfully closed tins." But, on inquiry, I was told that the number of unmistakable failures, even in the very best establishments, is very appreciable, and although many of these failures may be accounted for by clefective closure, $\mathrm{Mr}$ M'Call assured me that in a certain number of cases, where not the smallest defect could be detected in the tins, where the mode of preparation was unexceptionable, and the provisions originally of the best description, yet, for some reason unknown to him, some of these tins proved utter failures. Gas was found to be evolved within, causing them to bulge at the extremities, and, when opened, the meats either showed a central decomposition of a most fotid character without Mould, or else Mould might be found on some portions of the surface. In other instances, however, provisions would keep for ten years or more without any appreciable change; and I was informed that turtle, and all the other soups which solidified when cold, invariably remained good. This is only what might be expected, especially as Huizinga found that even too great a concentration of an ordinary infusion rendered the fluid less prone to undergo fermentative changes.

Further particulars on this subject may be found in Nature (Sept. 29, I 870, p. 433). It has only been referred to here because it has an important 
EXAMINATION OF PASTEUR'S DOC'TRINES II 5

bearing upon our experiments, and because the statements of Professor Huxley on the subject were so inexplicit as to be actually misleading, in more ways than one, as I then endeavoured to show. 


\section{CHAPTER XIII}

WAS PASTEUR RIGHT IN HIS EXPLANATION OF THE FACT THAT GUARDED NEUTRAL OR SLIGHTLY ALKALINE INFUSIONS PREVIOUSLY HEATED TO $100^{\circ}$ C. WILL OFTEN FERMENT?

GERHARDT says in his "Traité de Chimie Organique" (t. iv., I 856 , p. 547) : "Many substances which alone or in the moist state are not oxidised by the air, undergo such a process as soon as they come into contact with an alkali. Thus pure alcohol exposed to the air will remain pure indefinitely, and without becoming acid; but if a little potash be added to it, it speedily absorbs oxygen and becomes converted into vinegar and a brown resinous substance. It is clear from this that potash ought to favour certain fermentations, since it favours the absorption of oxygen, and the presence of this helps to develop ferments." He also says (loc. cit., p. 556), "It is known that meats and vegetable substances soaked in vinegar are preserved from decomposition, at least for a certain time. . . . The majority of acids produce the same effect as vinegar.'

Facts of this kind were doubtless well known to Pasteur, and he could also easily have satisfied himself concerning the relative amount of fermenI.6 
tative changes that would take place in otherwise similar slightly alkaline or acid organic infusions, even at ordinary temperatures and with more or less free exposure to the air. Thus, to quote one of the experiments made by myself many years ago, ${ }^{1}$ "Having prepared a pretty strong infusion of mutton, about an ounce and a half was put, after filtration, into each of two similar flasks. One portion of the infusion was allowed to remain neutral, while to the other three drops of strong acetic acid were added, so as to make the whole yield a faintly acid reaction to test paper. The two flasks were then exposed side by side to a temperature of $75^{\circ}$ to $80^{\circ} \mathrm{F}$. during the day. In twentyfour hours the neutral solution was clouded and more or less opaque, while the portion which was acid appeared perfectly unchanged. It was as clear as ever; and so it continued even to the end of forty-eight hours, although by this time the neutral solution was quite opaque and muddy-looking, with a pellicle on its surface, and also some flocculent deposit at the bottom of the flask." This fluid, on examination, was found to be swarming with Bacteria of different kinds.

Other experiments of a like kind, with different neutral or slightly alkaline infusions, have also shown that the addition of a few drops of acid causes the fluid, after a given time, to contain a notably smaller number of organisms than an equal bulk of the unaltered solution; and, conversely, having an acid infusion whose productiveness is known, the 1 "The Beginnings of Life," vol. i. p. 387. 
number of organisms found in equal bulks under similar conditions can almost always be very notably increased in either one of them by the mere addition of a few drops of liquor potassæ, so as to render that portion neutral or slightly alkaline.

Such facts may, of course, be interpreted as an indication that slight alkalinity or neutrality of the fluids is more favourable than their acidity for the occurrence of fermentative changes. Then, again, it would seem quite possible that the difference between acid and neutral solutions, as regards the number of organisms to be found in them when they have been simply exposed for a few days to ordinary atmospheric temperatures, might be exaggerated after such fluids had been subjected to the temperature at which water boils. It seems quite possible, that is, that high temperatures such as that of I $00^{\circ} \mathrm{C}$. might be more destructive to organic matter when contained in acid infusions than when it exists in neutral or slightly alkaline infusions. And other comparative experiments which I have elsewhere recorded ${ }^{1}$ show that this is so, and that the differences in the amount of fermentative changes to be seen at ordinary atmospheric temperatures are very distinctly exaggerated when other portions of the same neutral and acid fluids have been previously boiled.

Now let us look at Pasteur's experiences with acid and with neutral solutions, and at the degree

1 "The Beginnings of Life," vol. i. pp. 394-97. 
of thoroughness shown by him in seeking to interpret the different results met with.

The sub-title of his celebrated memoir of I 862 was, "Examen de la Doctrine des Générations Spontanées," and the first twenty pages of it were historical in nature, in which he discussed the experiments of Needham and Spallanzani pretty fully, and then those of Schwann and some other workers. His own researches had led him to believe that "ferments" were not albumenoid substances undergoing a peculiar though little-known change, as Liebig had taught. According to Pasteur (loc. cit., p. 23) mere albumenoid substances, "n'étaient jamais des ferments, mais l'aliment des ferments. Les vrais ferments étạient des êtres organisés." But it was impossible to establish this doctrine, or to attempt to establish it, except by rebutting the evidence which seemed to tell in favour of "spontaneous generation."

If, in such experiments as those he had been discussing, prepared after the manner of Needham and Spallanzani or after that of Schwann, the heating had been adequate and the subsequent protection from contamination had been perfect ; and if, nevertheless, organisms appeared within the experimental vessels, Pasteur ought to have known quite as well as either of these other experimenters, that if it could not be shown by direct experiments that the previous heating had been inadequate to destroy all pre-existing life within the vessels, the rival interpretation must be admitted, as Spallanzani fully recognised-namely, that there had been a de novo 
birth of living matter. Let us see, however, what Pasteur's attitude was in the face of such an eventuality.

The experiments of Schwann have been commonly believed by many to have been altogether in favour of the germ-theorists. Those who read his memoir will find, however, that he did not fail to obtain living organisms in all his experimental fluids. When the fluids were such as were capable of undergoing the alcoholic fermentation on exposure to the air, living organisms were, in spite of all precautions, sometimes found within his flasks. Many other observers have also found organisms in fluids from hermetically sealed flasks which had been strictly subjected to the conditions prescribed by Schwann; and that, not unfrequently, when the change which the fluid had undergone was of a putrefactive rather than of a fermentative character. Among those who have obtained these positive results may be named Mantegazza, Pouchet, Joly, Musset, Wyman, Hughes Bennett, Child, and others.

In spite of the fact that these investigators had obtained living things in vessels prepared after the manner of Schwann, when various infusions were employed, Yasteur was quite inclined to believe for a time, on the strength of his own experiments, that Schwann's precautions, properly carried out, were adequate to prevent the appearance of organisms in the experimental fluids. His early investigations were made with sweetened yeast-water, concerning 
which Pasteur said, "I have certainly had occasion to repeat the experiment more than fifty times, and in no case has this fluid, otherwise so changeable, shown a vestige of organism when in the presence of calcined air." But after a time Pasteur began to employ an entirely different fluid, and in all these experiments living organisms were invariably present in the previously boiled liquids, just taken from newly opened flasks. Formerly he used "l'eau de levûre sucrée," but now he employed milk-a much more complex and more highly nutritive fluid. After further experiments with this and other neutral fluids he came to the conclusion that living organisms might be encountered in almost any suitable neutral or slightly alkaline fluid, which had been submitted to Schwann's conditions; though, on the contrary, they were not to be met with when the solutions employed had an acid reaction.

Whatever fluids are used, if, after they have been boiled and exposed to a given set of conditions, organisms are not found, their absence would be explicable in one or other of two ways. Either the heat has proved destructive to all living things in these solutions; or else the particular conditions to which the organic matter in these solutions has been exposed have been such as to prevent the occurrence of fermentative changes. Any person wishing to ascertain the truth, and competent to deal with such a subject, could not fail to see that he was bound to give equal attention to each of these possibilities. He would have no right to assume that the probabilities were greater in favour of the one mode of 
explanation than in favour of the other. This it was which had to be proved.

But when positive results were obtained with milk or other neutral or slightly alkaline fluids, subjected to Schwann's conditions, the case became altogether different. The rule with regard to the inability of living things to survive in solutions which had been raised to $100^{\circ} \mathrm{C}$. for a few minutes was absolute, so far as it had gone, and had been founded on the best of evidence to which Pasteur as well as others had assented. No one therefore should have attempted to set it aside, except upon evidence equally direct and equally positive, though more extensive, than that upon which the rule had been originally founded.

Let us now see what was the course adopted by M. Pasteur. He explained the discrepancy between his earlier and his later experiments by at once assuming that the Bacteria and Vibriones which were ultimately found in the milk used in these later experiments had been derived from "germs" of such organisms which (contrary to the general rule previously admitted) had been capable of resisting the influence of the heat that caused the milk to boil. No direct proof of this assumption was ever attempted; yet Pasteur did not hesitate to proclaim that the "germs" of such organisms were not destroyed by the temperature of $100^{\circ} \mathrm{C}$. in neutral or slightly alkaline fluids (loc. cit.. pp. 5S-66), and that they were destroyed at I I ${ }^{\circ} \mathrm{C}$., simply because in dealing with such fluids, organisms were found after they had been heated to the one temperature, 
and were not found after they had been heated to the higher temperature.

He actually did not mention the other possibility, or attempt to adduce any direct and positive evidence in favour of his own view. Yet he might be supposed to have known such facts as are mentioned by Gerhardt in the quotation at the commencement of this chapter ; and he could scarcely fail to have seen that besides the mere difference in temperature as a cause of the different results with the same fluid, there was the possible influence of the mere slight acidity or alkalinity of the fluids in producing opposite results when the different fluids were heated to $100^{\circ} \mathrm{C}$. How easy would it have been to test the reality of this latter difference in the ways I have indicated, which show plainly enough that neutral or slightly alkaline infusions are the most favourable for the mere growth and development of Bacteria, and therefore might be more favourable also for Archebiosis. Origination and growth are, after all, possibly not so very different.

But not a word was said by Pasteur on this subject, nor was anything done by him to throw light upon the question. In fact he absolutely ignored the existence of one of the two possible interpretations of the problem under discussion. To come to the conclusion which he did, in face of what was previously known and assented to by himself as to the destructive influence of $100^{\circ} \mathrm{C}$. upon living matter immersed in fluids was, of course, absolutely unwarrantable. It will perhaps scarcely be credited by many that these particular 
investigations of M. Pasteur, which have had so much influence, and which have been looked upon by many as models of scientific method, should really contain such fallacies. ${ }^{1}$

M. Pasteur, in fact, entered upon a controversy concerning one of the most important questions in the whole range of biological science; and yet he assumed the attitude of a man who is so convinced beforehand of the error of those who are of an opposite opinion that he would not abide by ordinary rules; he would not entertain the possibility of the truth of the opinions opposed to his own. Ambiguous evidence was explaired as though it were not ambiguous; conclusions based upon good evidence were attempted to be set aside in favour of evidence which was comparatively worthless; and upon the strength of such illogical methods Pasteur proclaimed that he had "mathematically demonstrated" the truth of his own views. Still M. Pasteur's reputation as an exact and brilliant experimenter-true enough in many other most important researches-has been all-powerful, and the majority of readers have been only too willing to accept his conclusions on the origin-of-life question-conclusions which he had always so dogmatically proclaimed.

When Pasteur found that neutral or slightly alkaline fluids were so often fertile under conditions which left slightly acid fluids barren, before ignoring

${ }^{1}$ See p. 59 for what the writer in the Contemporary Reriew had to say concerning this mode of reasoning. 
the other possible explanation and jumping to the conclusion that the fertility of tile former fluids was due to the fact that Bacteria and Vibriones or their germs were able, in such fluids, to resist the destructive influence of $100^{\circ} \mathrm{C}$., it is clear that what ought to have been done was to institute direct experiments upon this question of the thermal deathpoint of the organisms in different fluids. But Pasteur never even hinted at the desirability of such a proceeding.

The reader will recollect, however, that I have made decisive experiments on this question, which have been recorded in Chapters ix. and $x$.

It was first shown that in an excellent saline nourishing fluid, having a neutral reaction (pp. 6o-62), Bacteria and Vibriones and their germs were killed by an exposure for about ten minutes to a temperature of $60^{\circ} \mathrm{C}$.

I subsequently showed that they were killed at about the same temperatures in organic infusions, and that certainly in such infusions, the thermal death-point never exceeded $70^{\circ}$. C. ; and further that no difference was met with when dealing with an acid turnip infusion or with a neutral or slightly alkaline hay infusion.

Similar temperatures were also shown to be fatal to Torulæ and Fungus spores generally.

There was then absolutely no foundation whatever for Pasteur's mere assumption that certain supposed "germs" of Bacteria and Vibriones were not killed in neutral or slightly alkaline fluids heated to $100^{\circ} \mathrm{C}$. For, seeing that the nourishing fluids 
employed were inoculated with a drop of a fluid in which myriads of Bacteria were multiplying rapidly, it must be supposed that they were multiplying in their accustomed manner, as much by the known method of fission as by any unknown and assumed mode of reproduction-that is by "germs," whether visible or invisible.

What has already been said would have constituted a complete answer to Pasteur at the time of the publication of his memoir, and for more than ten years later; because at that time the actual spores of Bacilli were unknown, and what Pasteur spoke of as "germs" of Bacteria and Vibriones were mere hypothetical bodies which neither he nor any one else had ever seen. At that period Bacteria and Vibriones were actually known to multiply only by processes of fission.

Later, in I 875 , the glistening and refractive spores of Bacilli were discovered, and much has been said concerning the powers possessed by these bodies of resisting heat, especially after a previous thorough desiccation. The extent of their powers in this direction has been since most carefully tested by me, as I have shown in Chapter $\mathrm{x}$. But, for very valid reasons, the nourishing fluid in which their powers of resistance were tested had an acid reaction, so that the results then arrived at cannot be quoted here in reference to the question dealt with in this chapter. Seeing, however, that Bacteria and Vibriones, which have not undergone a previous desiccation, have been shown to be killed (or at least 
to have had all their powers of growth and multiplication stopped) by a temperature of $70 \mathrm{C}$., and that this occurred just as certainly in neutral and slightly alkaline as in acid solutions, as shown in Chapter ix., it seems probable that the death-point for desiccated spores of these organisms might also not differ in nourishing fluids having these different reactions. Still this point has not been proved; so that, in the present state of knowledge, it must be admitted that, so far, an absolutely complete answer has not been given to what were unquestionably mere assumptions on the part of M. Pasteur. 


\section{CHAPTER XIV}

NEW EXPERIMENTS IN REFERENCE TO THE FERTILITY OF NEUTRAL ORGANIC SOLUTIONS HEATED TO $100^{\circ} \mathrm{C}$.

$\mathrm{T}^{\mathrm{T}}$ was long considered that temperatures between $177^{\circ}$ and $95^{\circ} \mathrm{F} .\left(25^{\circ}\right.$ and $36^{\circ} \mathrm{C}$.) were those most favourable for promoting fermentations. The upper limits of favourable temperature-what is now commonly spoken of as the "optimum temperature"had not been carefully defined; and this was the case especially in regard to the occurrence of fermentation in fluids which had been boiled. ${ }^{1}$

In previous experiments of this class no one had, so far as I am aware, designedly made use of a generating temperature above $100^{\circ} \mathrm{F} .\left(38^{\circ} \mathrm{C}.\right)$; the heat employed by some investigators has indeed been only too frequently below $77^{\circ} \mathrm{F} .\left(25^{\circ} \mathrm{C}\right.$. $)$. Previous to the month of August 1875, I had myself never purposely employed a generating temperature above $100^{\circ} \mathrm{F}$.; but early in that month I discovered that some boiled fluids which remained barren at a temperature of $77^{\circ}-86^{\circ} \mathrm{F}$., would rapidly become turbid and swarm with organisms if maintained at a

1 The first portions of this chapter are composed of extracts from a paper contained in the Joumal of the Linnacan Society (Zool.), No. 73, 1877, and No. 74, 1878 . 
temperature of I I $5^{\circ} \mathrm{F} .\left(46^{\circ} \mathrm{C}\right.$.). This important fact was ascertained while experiments were being made with hay-infusions and milk which had previously been subjected to destructive temperatures considerably higher than $212^{\circ} \mathrm{F}$.

Soon after, I discovered that an incubating or generating temperature as high as $122^{\circ} \mathrm{F} .\left(50^{\circ} \mathrm{C}\right.$.) may be had recourse to with advantage in dealing with some fluids. Organic infusions which would otherwise have remained barren and free from all signs of fermentation, have under its influence rapidly become corrupt and turbid. But although the high temperature proves to be so favourable for initiating chemical changes of a fermentative type in some organic fluids, it must not be assumed that it would be equally provocative in respect to all of them. The conditions most favourable for the initiation of such changes must be separately studied for each kind of fluid with which experiments are being made, since important specific differences may be encountered. I have already, however, ascertained that this high temperature of $122^{\circ} \mathrm{F} .\left(50^{\circ} \mathrm{C}\right.$. $)$ is just as favourable for the fermentation of milk and of hay-, turnip-, and other vegetable infusions, as it is for urine.

Shortly after my first announcement of this fact in June $1876,{ }^{1}$ it was made known by Professor Cohn ${ }^{2}$ that Dr Eidam had also discovered that certain organisms would grow and multiply rapidly at this high temperature in infusions of hay, though

1 Proceedings of the Royal Society, No. I72, p. I49.

2 Beiträge zur Biologie der PAanzen, Bd. 2, I876, p. 268. 
it was one which proved fatal to Bacterium termo, Torula, and other forms.

The powerfully stimulating influence of a temperature of $122^{\circ} \mathrm{F}$. may also be easily seen in another way. In addition to causing certain fluids to ferment which would otherwise remain barren at ordinary temperatures $\left(77^{\circ}-86^{\circ} \mathrm{F}.\right)$, it shows its influence upon those fluids which will ferment at these lower temperatures, by bringing about such a change with much greater rapidity.

No fluid serves better for showing these relative effects than urine which has been neutralised with liquor potassæ before the process of boiling, because, though it will mostly ferment at the lower incubating temperatures, it does so with difficulty and only after many days. Thus, I have found that a urine whose acidity required ten to twelve minims of liquor potassæe per ounce for neutralisation, would (after such admixture and an ebullition of five minutes' duration) not ferment under I 2 -I 5 days, if kept at a temperature of $70^{\circ}-73^{\circ} \mathrm{F}$., though such a change would show itself in $15-30$ hours at a temperature of $122^{\circ} \mathrm{F}$.

It was also in the summer of 1875 that I first made these experiments to ascertain whether, as with other acid fluids, the fermentability of urine would be increased by previously neutralising it with liquor potassæ. This preliminary inquiry was soon answered in the affirmative.

Then came the more important question as to the cause or mode of production of such increased fermentability. For two reasons urine seemed to 
me to be a fluid specially favourable for use in attempting to throw light upon this problem :first, because of the unanimity of experimenters as to the fact that, when boiled in its acid state and subsequently guarded, it invariably remained barren; and secondly, because the marked acidity of urine would necessitate the use of liquor potassæ in easily measurable quantities, even when providing for the neutralisation of such small portions of fluid as are commonly employed in these experiments.

The unanimity of previous investigators as to the barrenness of urine after it has been boiled in its acid state and subsequently protected from contamination, is remarkable, as may be seen from the following quotations.

M. Pasteur, speaking of sweetened yeast-water and of urine, says ${ }^{1}$ :- "Nous avons reconnu que ces liquides, portés à la température de l'ébullition à $100^{\circ}$ pendant deux ou trois minutes, puis exposés au contact de l'air qui a subi la température. rouge n'éprouvent aucune altération." The.latter of these fluids may remain, he says, indefinitely, "sans éprouver d'autre altération qu'une oxydation lente de la matière albumineuse," and this even "à la température de $25^{\circ}$ à $30^{\circ}$, température si favorable à la putréfaction de l'urine."

Professor Lister ${ }^{2}$ calls forcible attention to experiments with boiled urine in support of the germ theory-its continued barrenness, when protected

1 Ann. de Chimie et de Phys., i 862, t. lxiv., pp. 58 et 52.

2 Introductory Lecture, delivered in the University of Edinburgh, 1869, p. I9. 
after boiling, being the invariable result in his hands. In regard to any organisms and their germs which it might contain, he says :- "It is necessary to maintain the elevated temperature $\left(2 \mathrm{I} 2^{\circ} \mathrm{F}\right.$.) for about five minutes to ensure complete destruction of their vitality."

Dr (afterwards Sir) William Roberts ${ }^{1}$ mentions healthy and diabetic urine as being amongst the easiest fluids to sterilise, "three or four minutes' boiling" sufficing, as he says, to bring about this result and cause the liquid to remain permanently barren, when kept at temperatures ranging between $60^{\circ}$ and $90^{\circ} \mathrm{F}$.

Professor Tyndall ${ }^{2}$ also, in 1875 , found five minutes' boiling invariably sufficient to sterilise urine when it was subsequently exposed to a "moteless air."

This unanimity as to the barrenness of urine after a brief boiling in its acid state, together with the fact that, like other fluids, its fermentability was found to be increased after neutralisation by potash, seemed, as I have said, to make it a fluid specially favourable for trials as to the cause of the increased fermentability. Two alternative views were, of course, possible as to the cause of the fact in question--(I) It might be due to the "survival of germs" of some of the ferment-organisms in the boiled neutral infusions, as Pasteur assumed; or (2) it might be due to the mere chemical influence of potash in initiating and favouring the molecular

1 "Phil. Trans.," vol. clxiv. pt. 2, p. 46 г.

2 "Phil. Trans.," I \$76, vol. clxvi. pt. I, p. 42. 
changes leading to fermentation, as originally suggested by Gerhardt-and among these changes a process of life-origination.

This important question would seem to be so capable of being settled by crucial experimentation as to make it not a little remarkable that no such attempt was ever made by M. Pasteur. Thus, the fluids may be boiled in their acid state so as to kill all their contained germs and organisms, and to these fluids boiled liquor potassæ may be added in suitable quantity. ${ }^{1}$ The results of a number of such experiments should be sufficiently decisive to enable us to fix upon the true mode in which liquor potassæe operates in determining fermentation. If the fluids to which boiled potash is added in suitable quantity still remain barren, then such experimental results would unquestionably favour the first interpretation, viz., that given by M. Pasteur and adopted by other germ-theorists. If, on the other hand, the addition of the boiled liquor potassæ, to the urine which has been boiled in its acid state, suffices to convert this previously pure fluid into a turbid liquid teeming with ferment-organisms, then it would be conclusively shown that the increased fermentability of neutralised urine was ascribable to the second cause, viz., to the chemical influence of the liquor potassæ in initiating fermentative changes, whatever the precise nature of these early changes may be.

${ }^{1}$ A few experiments of this nature were first made by William Roberts with hay-infusion ("Phil. Trans.," I874, vol. clxiv. pt. 2, p. 474). 
Some preliminary experiments were made with an apparatus closely similar to that employed by W. Roberts in the very few experiments which he

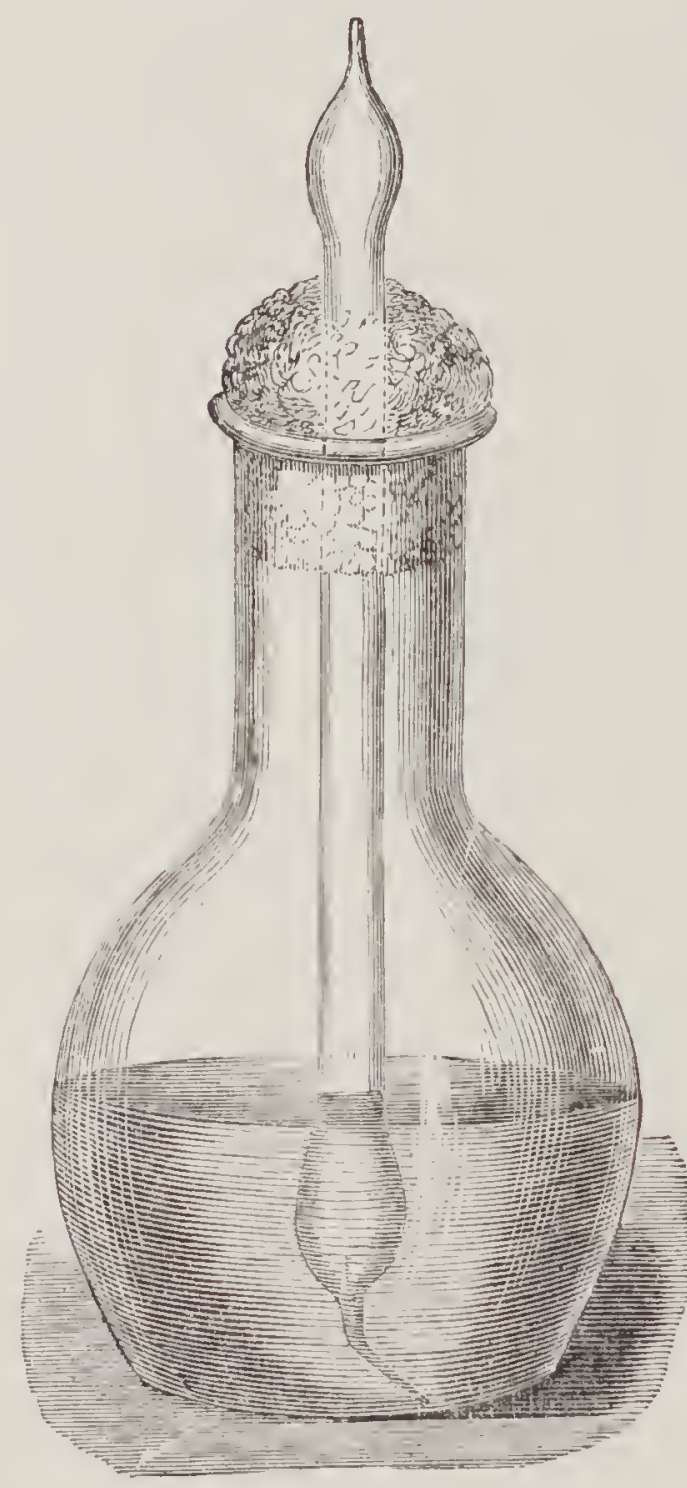

FIG. 2. undertook with hay-infusion. Small narrow-necked flasks were taken capable of holding nearly $3 \mathrm{oz}$. of fluid; and each of these was about half filled with a measured amount of fresh unfiltered urine, whose degree of acidity had been previously ascertained by carefully finding the exact number of minims of the liquor potassæ of the "British Pharmacopœia" which were needed to neutralise one ounce of it. ${ }^{1}$ Quantities of liquor potassæ, just sufficient to neutralise the amount of urine intended for each flask, were then enclosed in a number of Plugged Flask with liquor-potasse tube. glass tubes, each of which had a small bulb at one extremity, and a similar bulb near the other end, beyond which it was drawn out as a thin prolongation and bent at an obtuse angle. Each of these tubes was charged

I It may be well to mention that the solution of potash above named has a sp. gr. of 1058 , and that it contains 27 grains of caustic potash to the fluid-ounce of water $(5.84$ per cent.). What I have used has always been purchased from Mr Martindale, of Io New Cavendish Street, London. 
by heating it in a flame before immersing its open capillary extremity in the requisite quantity of liquor potassæ, contained in a minute porcelain capsule. When the whole of the measured amount of alkali had thus been forced into the glass tube, this was inverted, and its capillary extremity was sealed in the spirit-lamp flame. Its neck was then wrapped round with cotton-wool, and the tube itself was inserted into one of the flasks in such a manner that the cotton-wool might act as a plug thereto, while the capillary extremity of the tube just touched the bottom of the vessel.

The flasks being thus charged and arranged, the urine in its altered acid state was boiled over a flame for five minutes. When the fluid had cooled, the tube was pressed down slightly so as to break off its capillary extremity; and immediately afterwards a flame was applied to the external bulb of the tube, so as to expand its contained air. The measured amount of liquor potassæ was thus expelled into the sterilised urine; and the flask was then placed in an incubator and maintained at a temperature of $104^{\circ}$ - I I $3^{\circ} \mathrm{F}$. $\left(40^{\circ}-45^{\circ} \mathrm{C}\right.$. $) .^{1}$

Some tentative experiments were made in this manner with fresh urine, whose specific gravity varied from IO2O to 1025 , and whose acidity was such that 7-I 5 minims of liquor potassæe per ounce were required for neutralisation. In nearly every case it was found that the urine became lightercoloured and turbid in two or three days. Other

1 No higher incubating-temperatures were employed in these particular experiments. 
experiments showed that a slight excess of liquor potassæ tended to retard or even prevent the occurrence of fermentation, though a quantity of liquor potassa notably below that needed for neutralisation was found to be efficacious in inducing it, and that, too, almost as rapidly as if the neutralisation had been complete. Even when the liquor potassæ was added in quantity only sufficient for half-neutralisation, fermentation still took place in many instances, though in such cases the result was usually delayed for five or six days.

In all these trials it was found that the fluid, when turbid, was not fotid; its odour was for the most part scarcely at all altered, though at times it was rather more marked than usual. The organisms found in the fermenting urine were in all cases the same, viz. Bacilli, either short, medium size, or in the form of long threads (see Fig. 9) -and not the totally different minute ferment thought by Pasteur to be the invariable cause of the conversion, in urine, of urea into ammonic carbonate and water. ${ }^{1}$ Sometimes only the short unjointed rods were found, though more frequently these were mixed with varying amounts of longer Vibrio-like bodies, and with threads such as I and others have generally spoken of as Leptothrix.

The results of the foregoing preliminary experiments induced me to seek other, stricter methods, free from possible sources of fallacy which might be thought to have influenced the results.

1 Ann. de Chim. et de Phys., t. Ixiv. (I862), p. 50. 
To get rid, therefore, of all doubt concerning cotton-wool as a protective filter in such experiments, I determined to repeat the urine and liquorpotassæ experiments with hermetically sealed vessels from which air had been expelled by boiling, and to take the further precaution of boiling the liquorpotassæ tubes before inserting them into the experimental vessels. It was safe at once to resort to such a method, because I had previously ascertained that urine neutralised before boiling would ferment in such closed airless vessels almost, though not quite, as freely as in flasks plugged with cottonwool. There was, therefore, nothing unduly restrictive in the proposed conditions.

The new mode of procedure which I devised was conducted as follows :-

In the first place a stock of liquor-potassæe tubes had to be prepared beforehand containing convenient amounts of liquor potassæ. Some were charged with 8 , others with 10 , and others with I 2 or more minims. Those containing the same quantity were kept together in separate batches duly labelled and ready for use, as occasion required, according to the degree of acidity of the urine with which experiment was to be made. In order to ensure perfect accuracy in the measurement of the liquor potassæ, I made use of a small burette-tube (Fig. 3) graduated to minims and fitted with a stopcock, with which even half-minims could be delivered with ease.

Having prepared a number of small glass tubes closed at one end and drawn out at the other, I 


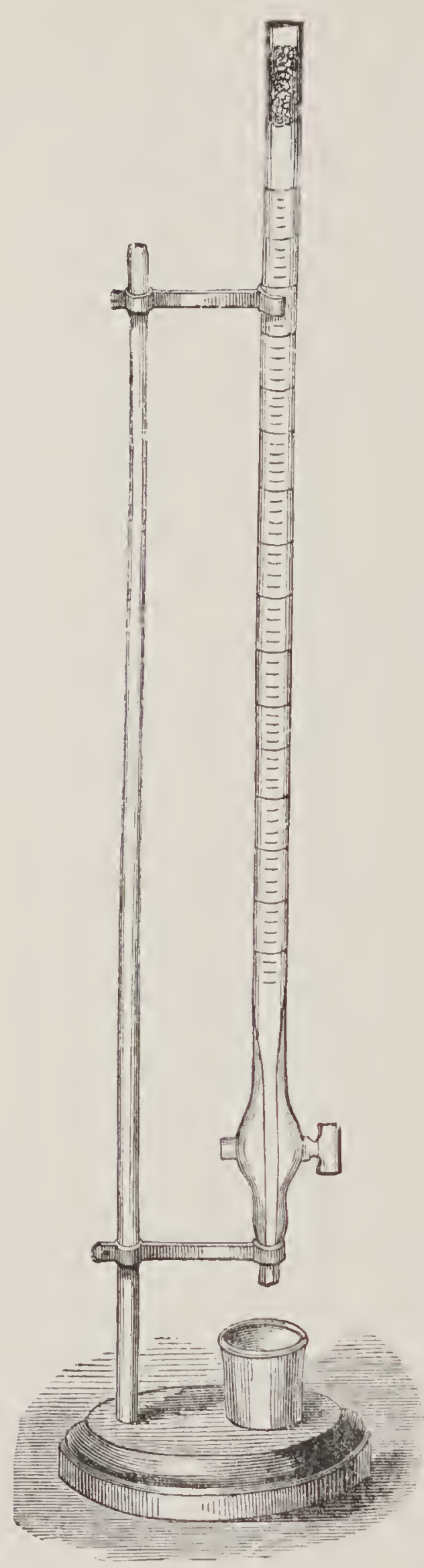

FIG. 3 .

Burette-tube for measuring liquor potassæ. proceed to charge them with measured amounts of liquor potassæ. As in the previous experiments, the liquor potassæ is delivered into a little porcelain pot; and the open capillary extremity of the glass tube, previously well heated in the flame of a Bunsen's burner, is immersed therein. When no more suitable rest is at hand the little porcelain vessel may be placed in the angle between two bottles, so that the upper end of the heated tube inclines against them, partly for support and partly that it may cool more quickly. In two or three minutes, when the whole of the liquor potassæ has been forced into the tube, this is to be inverted, and its shoulder, where it begins to narrow (Fig. $4^{*}$ ), is heated in a spirit-lamp flame, so that the tube may be drawn out still more in this situation. Subsequently the end of tube is bent, in the manner shown in the figure, and then hermetically sealed. 
Thus prepared, the tube should be just half full of liquor potassæ. Its length will have been diminished as much as possible; and its tip is so arranged that it may be easily broken off by a slight mechanical shock. These last steps in the preparation of the liquorpotassæ tubes are best carried out with the aid of a very small spiritlamp flame, as they require to be done slowly

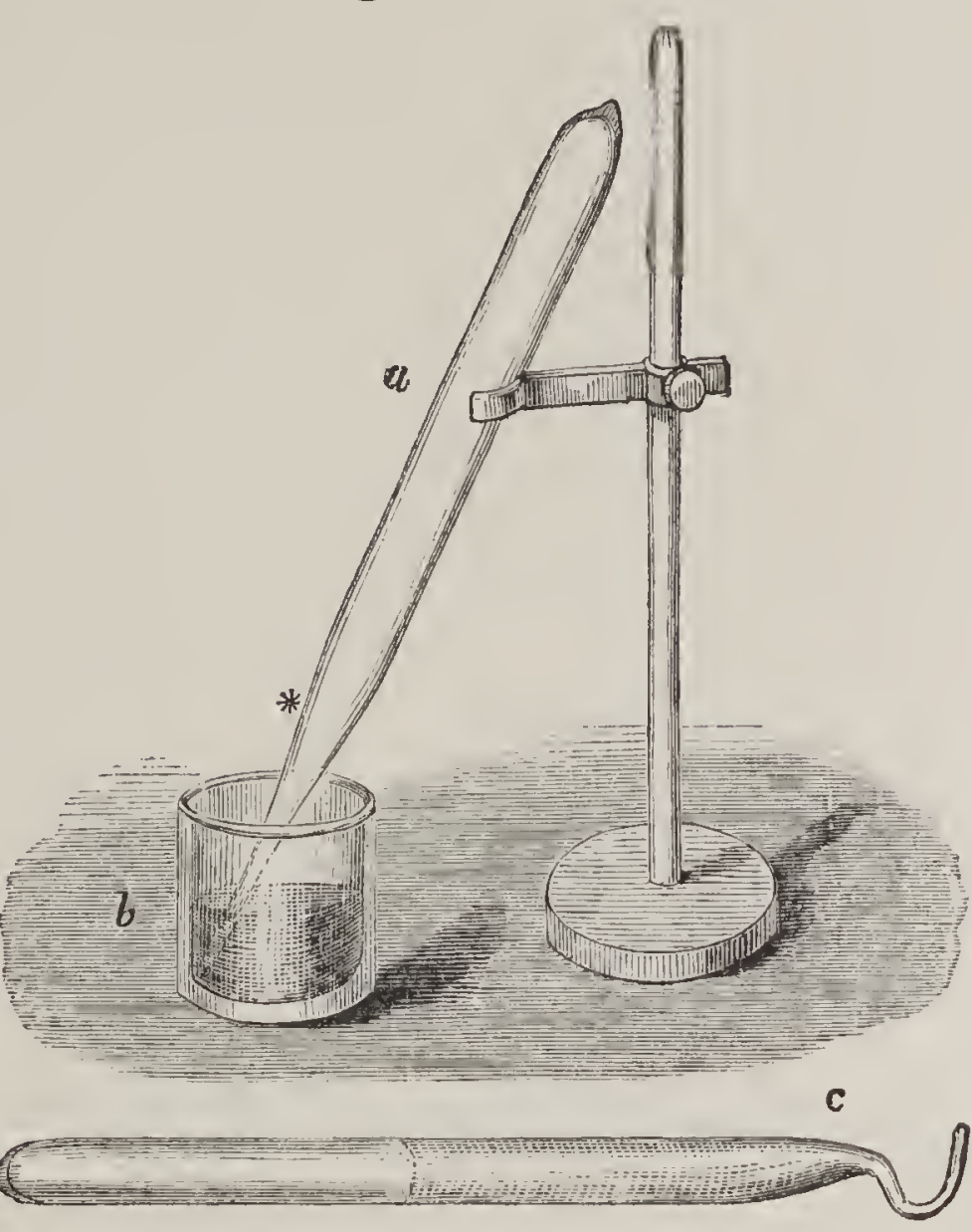

FIG. 4 .

Liquor-potassa tubes, with capsule and stand. and with care. On the one hand, it is necessary that the bent part of the tube should be weak enough to break readily when jerked against the wall of the experimental vessel in which it is afterwards enclosed; and, on the other, it must not be so much weakened as to make it break too easily or be unable to bear the internal strain which it will have to undergo during its immersion in boiling water. This, in fact, is the final stage in the preparation of the liquor-potassæ tubes. A number of them, after they have been hermetically sealed, are to be placed in a suitable vessel containing warm water; and they are then to 
be raised to the temperature of $212^{\circ} \mathrm{F}$. $\left(100^{\circ} \mathrm{C}\right.$.) for the period fixed upon. As in these experiments I soon found that the longer or shorter duration of the period of boiling of the liquor-potassæ tubes did not appreciably influence the results, they were, for the most part, boiled for $15^{\prime}-20^{\prime}$ only-though in many cases it was much longer, and on two or three occasions they were boiled for two hours. Thus prepared, the tubes were set aside in compartments labelled according to the number of minims of liquor potassæe which they contained.

A stock of such tubes being ready to hand, experiments may be made at any time. A suitable specimen of fresh urine is to be taken, whose specific gravity is to be ascertained and whose degree of acidity is to be most carefully estimated. This latter process I have carried out by taking exactly one fluid-ounce of the urine and adding liquor potassæ to it, minim by minim, from the burettetube till the point of saturation is nearly reached. Thereafter, the alkali has been added in half-minims at a time, and tested between each addition with litmus and turmeric paper so as to make quite sure of the time of complete neutralisation. ${ }^{1}$ In order to facilitate this part of the process, I have made use

${ }^{1}$ It is not unimportant here to add that the test-papers which I have used have been those sold by Mr Martindale, of Io New Cavendish Street, London. They are similar to the papers used in the wards of University-College Hospital. By careful trial I have ascertained that $\frac{1}{8}$ of a minim of liquor potasse in an ounce of distilled water may be recognised by the previously reddened litmus paper, while $\frac{1}{4}$ of a minim in the same quantity of distilled water may be detected by the yellow turmeric paper. The latter, though less delicate, gives the most certain indication, especially when a drop of the fluid to be tested is 
of a lipped measure (Fig. 5) having a rather narrow orifice, which can be easily covered by the thumb so as to allow its contents to be shaken for the thorough admixture of each quantity of liquor potassæ with the urine to which it has been added.

These experiments have hitherto been exclusively conducted with my own urine; and I have generally found that which was passed in the morning before breakfast very suitable for use. This fluid has remained clear after boiling, no phosphates being deposited during the process; its acidity has usually been neutralised by IO-I4 minims of liquor potassæ per ounce; and its specific gravity has varied from 1020 to 1025 .

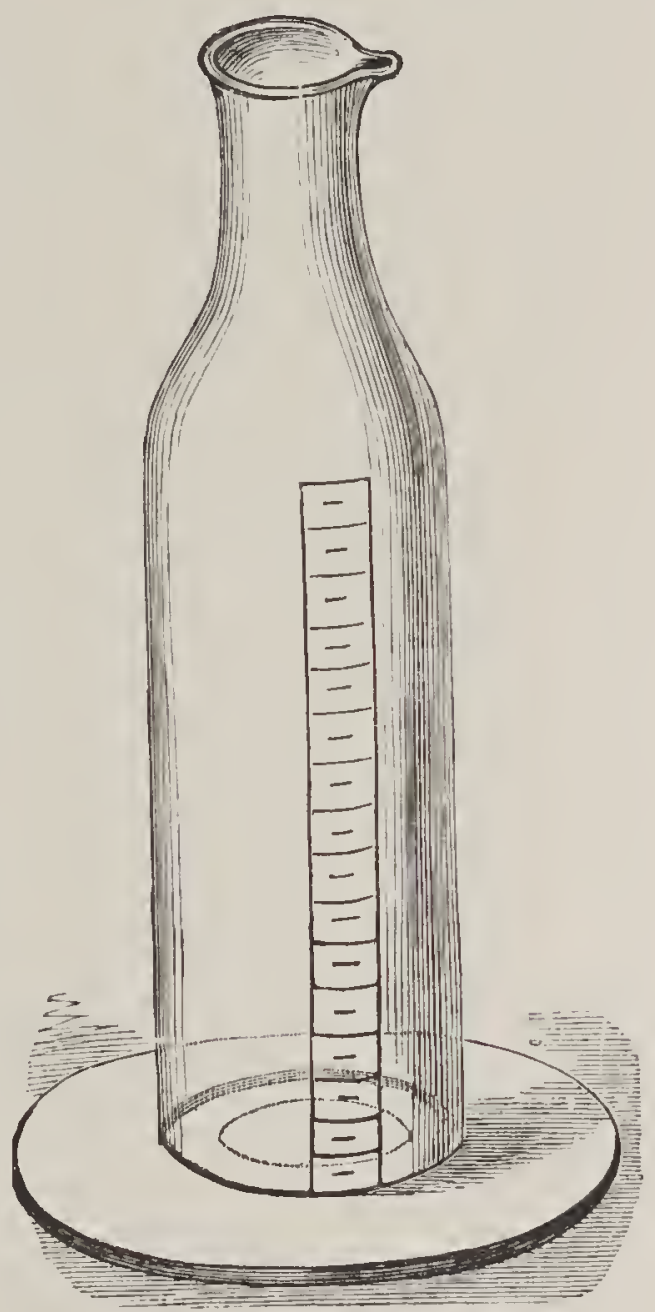

FIG. 5 .

Lipped measure for admixture of liquor potassæe with urine.

When the acidity of the urine with which experiment is to be made has thus been accurately determined, one can easily settle which set of the already prepared liquor-potassæ tubes it will be most convenient to employ. I have generally made use of about one ounce of urine for each experiment; and, after numerous trials, have found it best in this

allowed to fall on dry turmeric paper. As the fluid is absorbed by this partly bibulous paper, a faint brown circle is seen for a moment or two when the fluid is very faintly alkaline. 
series not to provide liquor potassæ sufficient to exactly neutralise the quantity of fluid in the unboiled state, but rather to provide it (in a closed tube) to the extent of two-thirds or three-fourths of this amount-the former being, on the whole, the safest proportion. ${ }^{1}$

An illustration will make the mode of procedure, at this stage, clearer. If the urine to be employed has an acidity of 12 minims of liquor potassa to the ounce, then I ounce of it should be placed in each experimental vessel, and with it a liquor potassæ tube containing 8 minims of this fluid-inserted, with its narrowed and bent extremity downwards. If, on the other hand, the urine had an acidity of 8 minims of liquor potassa to the ounce, and only tubes containing this amount of liquor potassæ were at the time available for use, we should then have to place in each experimental vessel $\mathrm{I}_{2}^{\frac{1}{2}}$ ounce of the urine and one of these 8 -minim tubes.

When properly charged, the neck of the retort or flask is to be heated and drawn out to a narrow extremity; after which the urine is gently boiled for about two minutes over a flame, great care being taken to avoid any waste of the fluid by spurting. During the continuance of ebullition the extremity of the vessel is hermetically sealed.

1 There is reason to believe that conditions other than the acidity of the fluid may subsequently have to be taken into account, since, although $\frac{2}{3}$ may seem quite favourable for one specimen, in another $\frac{3}{ \pm}$ of the amount of liquor potassæe which would have been requisite for full neutralisation before boiling appears to produce more speedy results. Such, or analogous, differences may also have to be ascertained in regard to the urine of different individuals. 
Some little practice is required to do this properly - that is, on the one hand to seal the vessel while

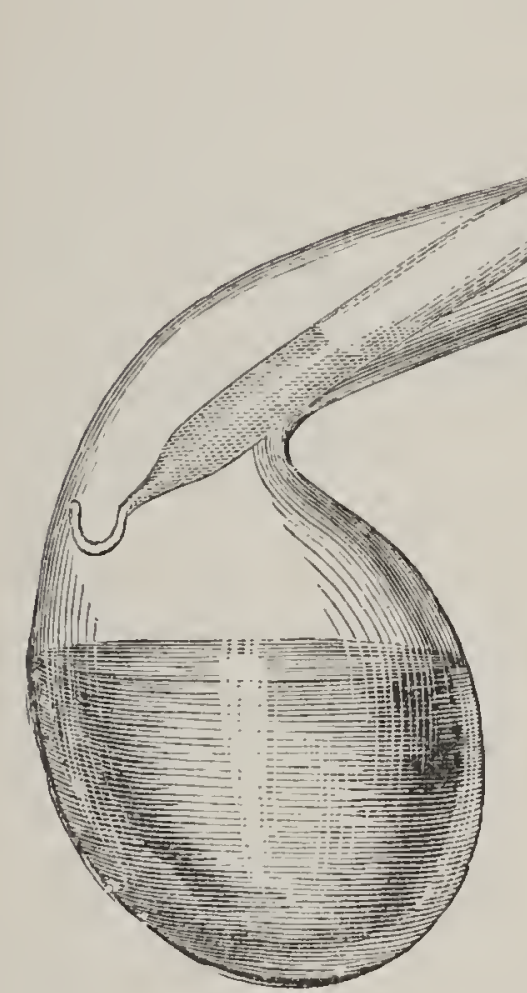

FIG. 6.

Sealed Retort containing liquor-potassæ tube.

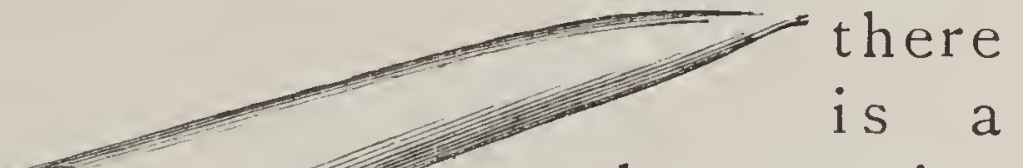

gentle outpouring of steam, and, on the other hand, to do it in such a way that there is no inbending of glass at the sealed extremity. Even a small amount of such inbending is very apt to lead to a minute crack at the next stage. After allowing an interval of $\frac{3}{4}$ of a minute for the sealed tip to cool a little, the retort is inverted, and in this position is at once immersed in a can of boiling water, prepared and ready to hand for this purpose. Here the experimental vessel is left for 8 minutes or more.

Three purposes are served by this double process of heating. In the first place, it simplifies the experimental conditions to get rid of the air by boiling; secondly, the speedy closure of the vessel and the prolongation of the heating in a can of boiling water reduces the loss of fluid by boiling to a minimum; and thirdly, and principally, the inversion of the experimental vessel during the second period of heating brings those upper portions of the internal surface, as well as the outer surface, of the liquorpotassæ tube (which, during the boiling over the 
flame, may only have come into contact with steam at $2 \mathrm{I} 2^{\circ} \mathrm{F}$.), into continuous contact with the heated fluid itself. ${ }^{1}$

After the urine in the boiled retort has become cool, the liquor potassæ is allowed to mix therewith. This is easily brought about by shaking the retort or flask so as to jerk the bent capillary extremity of the liquor-potassæ tube against its internal surface. The neck of the previously closed tube is thus broken off, and the liquor-potassæ itself, owing to the comparative vacuum within the experimental vessel, is forced out and mingles with the sterilised acid urine.

If six to ten vessels have been prepared in this way, charged from the same stock of urine, some one or two of them may be selected for "control" experiments. In these the liquor-potassæ tubes are not broken, while in all the others they are; so that when subsequently placed in the incubator together at a temperature of $122^{\circ} \mathrm{F}$., the two sets of retorts constitute crucial experiments capable of testing the influence of liquor-potassæ upon the sterilised urine.

What I have found to happen almost uniformly in about two hundred of such experiments is this: If suitable fluids are dealt with-that is, specimens of fresh urine whose acidity before boiling does not require less than 8 minims of liquor potassa per ounce for neutralisation, and which do not deposit phosphates on boiling-the urine in the control ex-

1 The whole of the internal surface of the liquor-potassæ tube is similarly exposed to the influence of its heated and caustic fluid during these different modes of heating. 
periments remains clear and apparently unaltered for an indefinite time; while where the potash has been allowed to operate upon the sterilised fluid, it becomes turbid, lighter in colour, and swarms with organisms, on an average, in from i 8 to 36 hours. The period, with different urines, is sometimes less and sometimes more, though no great prolongation occurs except through some alteration having been brought about in the proper ratio that should exist between the acidity of the boiled fluid and the amount of liquor potassæ which is added thereto. Such delays in the occurrence of fermentation were common enough during my earlier trials with this method; but now that I have more carefully studied and ascertained some of their causes, I am generally able to obviate them and ensure the supervention of fermentation within two or three days.

This fermentation of urine to which liquor-potassa is added after boiling, unquestionably takes place more readily in a flask plugged with cotton-wool than in a sealed retort from which air has been expelled by boiling. And that the slightly diminished readiness of the fluid to ferment in the airless retort is attributable to the absence of atmospheric oxygen, seems to be confirmed by other experiments, in which an increased readiness to change is exhibited by urine and liquor potassæ under the influence of nascent or less-diluted oxygen, liberated by electrolysis - the retorts in this case being provided with platinum electrodes. ${ }^{1}$

I have also in two experiments with closed flasks 1 Loc. cit. pp. 9-12. 
containing urine and ordinary atmospheric air ${ }^{1}$ liber? ated the liquor potassæ after the flask and its con.

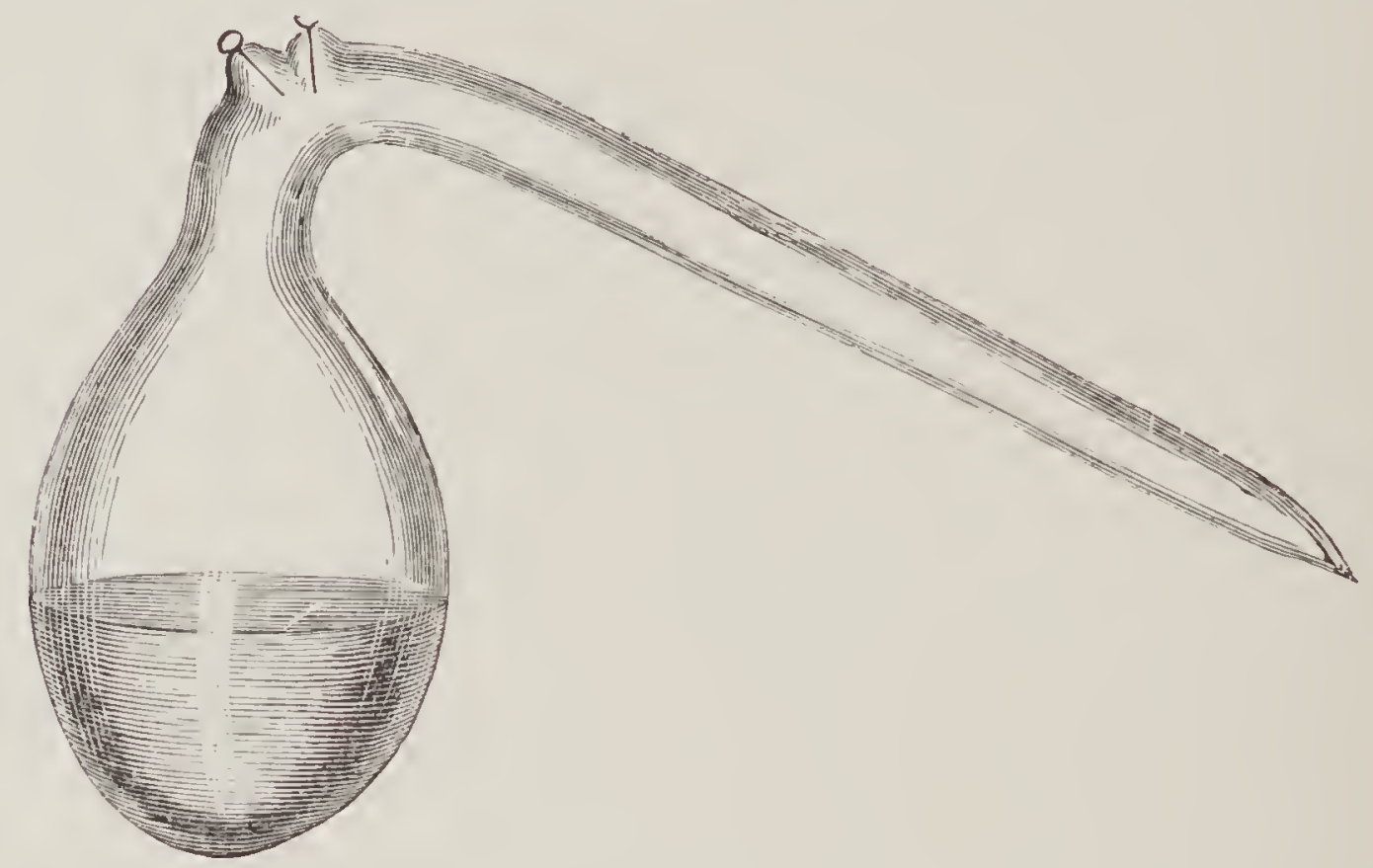

FIG. 7 .

Retort with platinum electrodes for the liberation of oxygen.

tents had been boiled, with the effect of finding fermentation take place several hours earlier than it did in other companion retorts whose contents were similar, except for the fact that they contained no atmospheric air. The liberation of the liquor potassæ from the tube with a capillary neck can only be brought about with great difficulty in a retort containing air ; and this was the reason of my giving up the conditions more favourable to the fermentation of the boiled urine, in order to avail myself of the facile and automatic emptying of the liquorpotassæ tube that takes place in the retort from which air has been expelled by boiling. ${ }^{2}$

1 The flasks having been hermetically sealed while the urine was cold.

2 The first account of these experiments is to be found in The Proceedings of the Royal Society, No. 172 (June 1876 ); and there is also 


\section{The Question of the Interpretation of these Experiments.}

All other experimenters had found, as I have shown, that acid urine after it had been boiled for a few minutes, and subsequently guarded from contamination, remained barren; and the explanation given by all was that all living things had been killed in the urine by the temperature of $100^{\circ} \mathrm{C}$.

The fact, therefore, of the fermentation of some specimens of boiled acid urine, with the appearance of swarms of Bacteria therein, under the influence of the high incubating temperature of $122^{\circ} \mathrm{F} .\left(50^{\circ} \mathrm{C}\right.$.), seems inexplicable except upon the supposition that fermentation has, in these instances, been initiated without the aid of living germs, and that the organisms first appearing in such fluids have been evolved therein.

If my further position (Proceedings of Royal Society, Nos. 143 and 145,1873$)$ that Bacteria and their germs are killed in fluids, whether acid or alkaline, at a temperature of $158^{\circ} \mathrm{F} .\left(70^{\circ} \mathrm{C}\right.$. $)$ is correct, then the occurrence of fermentation in the previously neutralised boiled urine would similarly disprove the exclusive germ-theory of fermentation, and establish the doctrine of Archebiosis.

Any difficulty which might have been felt by others in accepting the above interpretation of the

a fuller account, with many more details, in the Journal of the Linncean Society (Zool.), Nos. 73 and 74 (October 1877 and May I878), extracts from which I have been reproducing here. 
results of these latter experiments-in face of the view held by M. Pasteur that some Bacteria germs are able, in neutral fluids, to survive an exposure to a heat of $100^{\circ} \mathrm{C}$.-would seem to have been fairly met and nullified by the experiments just recorded and devised for the purpose, in which the urine was boiled in the acid state so as to kill all pre-existing living things, and was subsequently fertilised by the addition of boiled liquor potassx.

If we look at these latter experiments from an independent point of view, it will be found that this fertilisation of a previously barren fluid by boiled liquor potassæe can only be explained by one or other of three hypotheses:-

ist Hypothesis. The boiled liquor potasse may act as a fertilising agent because it contains living germs. However improbable this hypothesis may seem on the face of it, looking to the caustic nature of the fluid and its solvent action on protoplasm, it has been actually disproved by many of the experiments made during this investigation. These experiments have shown that boiled liquor potassæe will only act as a fertilising agent when it is added in certain definite proportions. If it acted as a germ-containing medium a single drop of it would act just like a single drop of tap-water, and would suffice to infect many ounces of the sterilised fluid. This, however, is never the case; the liquor potassæ only fertilises the barren wine when it is added in a proportion which shall not be more than just sufficient to neutralise the urine at the time when it is added. 
2nd Hypothesis. The fertilising agent may act by reviving germs hitherto presumed to have been killed in the boiled acid urine. The acceptance of this hypothesis would involve a general recantation of the previously received conclusion that Bacteria and their germs are killed by boiling them in acid urine, as well as in other acid fluids, such as Pasteur's sweetened yeast water, which remain barren after boiling. But no such recantation of opinion would be justifiable except it could be based upon good, direct, and independent evidence.

The possibility, however, of accepting this second hypothesis seems still further closed by the results of experiments in which a slight excess of liquor potassæe was added to the boiled urine. Such fluids invariably remained barren. Yet it can be easily shown that the mere development and growth of Bacteria germs may take place both quickly and freely in boiled urine containing a very large excess of liquor potassæ. ${ }^{1}$ Thus, it would seem that this agent, mixed with boiled urine, in quantity slightly more than is needed for neutralisation, prevents the generation of living matter therein, although, even when in considerable excess, the same agent affords no obstacle to the development, growth, and multiplication of germs purposely added thereto-the mixture, in fact, proves a good "nourishing" liquid.

1 Thus, if one ounce of urine may be neutralised by ten minims of liquor potassa, we may add sixty minims of this fluid to one ounce of the same boiled urine; we may pour it into a corked phial which has been washed with tap-water, so as to contaminate it, and within forty-eight hours the fluid will swarm with Bacteria if it is kept at a temperature of $122^{\circ} \mathrm{F}$. 
3rd Hypothesis. The fertilising agent acts by helping to initiate chemical changes of a fermentative character in a fluid devoid of living organisms or living germs. If fermentation occurs in the absence of living units, then it must be occasioned by some chemical reactions set up between the boiled liquor potassa and the boiled urine; and these experiments would seem to prove that living matter may and does originate independently during the progress of fermentation in certain fluids-since, in the experiments in question, swarms of Bacteria make their appearance in the fluids employed. 


\section{CHAPTER XV}

DISCUSSION WITH M. PASTEUR IN REFERENCE TO THE EXPERIMENTS RECORDED IN THE LAST CHAPTER, FOLLOWED BY THE APPOINTMENT OF A COMMISSION BY THE ACADEMY OF SCIENCES OF PARIS

ON July IO, 1876, an abridged account of the experiments recorded in the last chapter was read before the Academy of Sciences of Paris ; ${ }^{1}$ this brought a reply from M. Pasteur on the following Monday, which was the beginning of a rather long discussion between us. Before referring to this discussion in some detail, it will be convenient first to refer to two attempts, made shortly afterwards in this country, to repeat my experiments.

In the month of December two communications were sent to the Royal Society by Dr (afterwards Sir) William Roberts and by Professor Tyndall, giving an account of what they considered to be repetitions of my experiments. They, however, introduced two modifications, one of which so altered the conditions prescribed by me as to make it certain that their experiments would lead, as they did, to negative results.

${ }^{1}$ A longer abstract of the original memoir had been published in Nature on July 6 th, 1876 . 
The first modification was a super-heating of the potash-tubes. To this I had no objection, and as it will be fully referred to in my discussion with $\mathrm{M}$. Pasteur, nothing need be said on that subject now.

The second modification was this : liquor potassæ was added in quantity sufficient to "exactly neutralise" the urine employed; this was then boiled over the flame for five minutes before the neck of the flask was sealed. The flasks were "then set aside in a warm place $\left(70^{\circ}\right.$ to $80^{\circ} \mathrm{F}$.) for a fortnight," and Dr Roberts says, the liquor potassæ was not added "till the lapse of time had satisfied me that it had been rendered permanently barren." Professor Tyndall says that in these respects "the procedure described by Dr Roberts was accurately pursued" in his own experiments. And he says at the close of his brief communication, "The experiments have already extended to one hundred and five instances, not one of which shows the least countenance to the doctrine of spontaneous generation." This I can readily believe, as the methods adopted by these experimenters certainly entailed the addition of liquor potassæ considerably in excess of the proper amount, and the method adopted by them was also wrong in other ways.

In the first place there was no necessity to depart from the ordinary method of the "control " experiment-especially in dealing with such a fluid as boiled acid urine which Dr Roberts and Professor Tyndall, as well as other experimenters, had declared to be invariably sterilised by a brief elevation to $100^{\circ}$ C. $($ see p. 132$)$. 
They began by providing liquor potassæ sufficient for accurately neutralising the quantity of urine employed (instead of three-fourths, of that amount, as I advised); they boiled the urine over the flame for five (rather than for one or two) minutes, a procedure which was found by Pasteur, as well as by myself, to lead to a decomposition of urea into ammonium carbonate; and then the closed flasks were kept for fourteen days in a "warm place," during which time, as I have fully shown elsewhere, a still further reduction in the acidity of the urine would go on, though more slowly. The combined effect of providing too much potash in the first place, of diminishing the acidity of the urine by the more prolonged heating over the flame, and still further by keeping it in a warm place for a fortnight before the liquor potassæ was liberated from the little tubes, was such as inevitably to lead to the cause of failure to which I had specially called attention. Though probably neither of these experimenters knew about the lowering of the acidity of the urine by the boiling and during its subsequent stay in the warm place, full details on this subject are to be found in my memoir in the Journal of the Linnean Society (Zool.), vol. xiv. pp. 28-43, which was published in the following yearsome extracts from which on this subject are to be found in the next chapter (pp. I70-I73).

Having shown the reason why Sir William Roberts and Professor Tyndall failed in their attempts to repeat my experiments, we may now turn to what M. Pasteur had to say concerning them. 
Unlike the English investigators he did not contest the facts. He said, "Je m'empresse de déclarer les experiences de M. le Dr Bastian sont, en effet, très exactes; elles donneut le plus souvent les résultats qu'il indique." It was in fact the interpretation only which he then contested; as he declared that the experiments were of the same order as some which he published in 1862. The fact that the urine had been boiled by me in the acid state being rendered nugatory, as Pasteur thought, because the liquor potassæ had not been heated to IIO C.; and because, as he thought, this fluid contained living germs. He begged me to repeat the experiment, using only liquor potassæ which had been heated to $\mathrm{IIO}^{\circ} \mathrm{C}$.

On July 3I I replied that very many experiments had shown me that the liquor potassæ only fertilises the urine when it is added in quantity not more than sufficient to neutralise the quantity of urine employed at the time of its addition. And I asked him to give some direct proof that germs of Bacteria could survive in a fluid so caustic as the liquor potassæ of the British Pharmacopeia, even for an instant, when raised to a temperature of $100^{\circ} \mathrm{C}$. As he did not think the high incubating temperature of $50^{\circ} \mathrm{C}$. was necessary, I replied that I considered it of much importance, as under its influence fluids would often ferment in a few days which at lower incubating temperatures would have remained sterile.

In his rejoinder on August 7, Pasteur again repeated that he did not contest my facts, still declar- 
ing that my experiment was only a reproduction under another form of what he had shown in 1862 ; and that we only differed as regards the interpretation to be given to it. He made no reply to my request for direct evidence that germs could survive in liquor potassæ raised to $100^{\circ} \mathrm{C}$., even for an instant. In repeating my experiment he rendered the urine "alkaline," as he said, by the addition of solid potash. He concluded by telling the Academy that my interpretation was "absolutely erroneous," while his was "incontestably established."

On August 2 I I pointed out that in rendering the urine "alkaline" by solid potash, he had probably added too much, and thus had failed to obtain fertility-as even a slight excess almost always resulted in the urine remaining sterile. I reported that I had used liquor-potassæ tubes which had been heated to $110^{\circ} \mathrm{C}$., and that they were just as potent as if they had only been heated to $100^{\circ} \mathrm{C}$. The fact, indeed, that too little or too much liquor potassæ heated only to $100^{\circ} \mathrm{C}$. left the urine sterile showed clearly enough that the liquor potassæ was a germless fluid. It was useless, therefore, further to press M. Pasteur for direct proofs (which he did not attempt to give) that such a fluid heated to $100^{\circ} \mathrm{C}$. could contain living germs. ${ }^{1}$

No reply came for many months, but in the

1 In reference to Pasteur's contention that liquor potassæ raised to $100^{\circ} \mathrm{C}$. contains living germs The Lancet said in a leading article (February 17,1877 ): "It is difficult to conceive a weaker objection to the above experiments than the assertion that living things can survive boiling in liquor potassæ, a reagent which in the cold state dissolves and destroys protoplasm." 
Comptes Rendus for January 8, i 877 , a communication appeared from MM. Pasteur and Joubert, ${ }^{1}$ saying they had again gone carefully over the questions in dispute, but that for the present they wished the discussion limited to the one point, whether pure solid potash or a potash solution heated to $100^{\circ} \mathrm{C}$. would fertilise boiled urine. They still implied that living germs were contained in boiled liquor potassa; and in reference to his own negative results, Pasteur said the question is limited to thisHave I passed the point of saturation (that is, made the urine "alkaline"), and if so, is there any harm in so doing?

On January 22 I could only reiterate what I had said before: that the use of solid potash was altogether unnecessary; that the solution could be easily heated to $110^{\circ} \mathrm{C}$. and would act just as if it had been heated only to $\mathrm{I}^{\circ} \mathrm{C}$., when in suitable quantity; that there was absolutely no evidence of the existence of living germs in liquor potassæ heated to $100^{\circ} \mathrm{C} .{ }^{2}$ and the proof of this was that too much or too little potash contained in the tubes had no fertilising effect. That was the proof of my position, though he could produce no independent evidence of a contrary kind. Of course, if the boiled liquor potassa contained living germs, one or two drops of it would act just like tap water and contaminate

1 The latter being Professor of Physics in the College Rollin. His help was also acknowledged by Pasteur in his first reply to me.

$2 \mathrm{Or}$ in the air contained with the boiled potash tubes, which was what Wm. Roberts suggested. It was this supposition that led him to advise their superheating. He did not venture to suggest that germs could survive in the boiling liquor potassæ! 
any quantity of boiled urine, and $\grave{a}$ fortiori so would an excess of the potash. So that my reply to his questions was: Yes, you have probably added too much potash (in making the urine "alkaline"), and that is probably the cause of your uniform failure to obtain fertility.

I concluded by saying further discussion seemed useless, but that I was willing to repeat my experiments before competent witnesses.

This drew from Pasteur a characteristic reply on the following Monday; so erroneous in regard to fact, and dogmatic in tone, as to deserve reproduction in full, with italics as in the original note. It runs as follows :-

"M. le Dr Bastian, répondant a la Communication que j'ai faite le 8 janvier en collaboration avec M. Joubert, a adressé à l'Académie, lundi dernier, une longue Note où il s'est encore appliqué, suivant moi, à éluder le point vif du débat. Dans notre Communication du 8 janvier, il y avait un mot d'une signification capitale: c'était celui de potasse pure. Or, chose surprenante, dans la réponse de trois pages du Dr Bastian, il n'y a pas même une allusion a cette condition de pureté, qui était tout.

"Je vais faire une nouvelle tentative pour ramener le savant anglaise a ce criterium, auquel il ne saurait échapper, quoi qu'il fasse.

"La discussion a été soulevée par cette affirmation qui lui est propre: Une solution de potasse bouillie fait naître des bactéries à 50 degrés dans l'urine stérile, après qu'on l'a ajoutée à celle-ci en quantité voulue pour la neutralisation exacte.

"Voici ma réponse au savant professeur d'Anatomie pathologique de Londres:

"Je mets au déf le Dr Bastian d'obtenir, devant des juges compétents, le résultat que je viens de rappeler, avec de l'urine stérile, a la seule condition que la solution de potasse qu'il emploiera sera pure, 
c'est-à-dire faite avec de l'eau pure et de la potasse pure, Iune et l'autre exemptes de matières organiques. Si le Dr Bastian veut se servir d'une solution de potasse impure, je l'autorise encore parfaitement à la prendre telle et quelconque, dans le pharmacopée anglaise ou ailleurs, très-diluée ou concentrée, à la seule condition que cette solution sera portée préaloblement à I $\mathrm{I}$ o degrés pendant vingt minutes ou a $\mathrm{I} 30$ degrés pendant cinq minutes. ${ }^{1}$

"C'est assez clair, ce me semble, et M. Bastian me comprendra cette fois."

On February I 2 I replied that I had accepted his challenge, and that I had repeated my experment first with liquor potassæ heated in a tube to I I ${ }^{\circ} \mathrm{C}$. for sixty minutes, and then with some which had been raised to $110^{\circ} \mathrm{C}$. for twenty hours, with results similar to those that $I$ had obtained when the liquor potassæ tubes had been heated only to $100^{\circ} \mathrm{C}$., and added in suitable quantity: that is to say, in twenty-four to forty-eight hours the urine was in full fermentation and swarmed with Bacteria.

M. Pasteur thanked me for accepting his challenge, and begged the Academy to nominate a Commission "to report upon the fact" which had been under discussion between Dr Bastian and himself. And in the next number of the Comptes Rendus there appeared an announcement that "MM. Dumas, Milne Edwards, and Boussingault sont designés pour constituer la Commission qui sera appelée à exprimer une opinion sur le fait que est en discussion entre M. le Dr Bastian et M. Pasteur." I wrote to M. Dumas on February 27, saying; "If a convenient time can be arranged I shall be very happy to come to Paris for three days, in order to perform my ex-

1 All italics as in the original. 
periments before the Commission which has been nominated by the Academy."

Several letters subsequently passed between M. Dumas and myself, which together with an account of what actually occurred at the meeting of the Commission are to be found in Nature for August 2, I877. I shall, therefore, only give here an abbreviated account of this correspondence.

Owing to my not having received a letter sent by M. Dumas on April 25 (which was subsequently forwarded in duplicate), I actually heard nothing from him till a letter was received dated May 5, saying that M. Pasteur had already performed his experiments before the Commission, that they were ready to receive me at any time, and placing the laboratory of the École Normale at my service.

I subsequently wrote to say I should be unable to come to Paris till "the third week in July when our medical session will terminate," and wished again to ascertain exactly what the programme of the Commission was to be. Later, I learned that "the Commission will allow to each of us the opportunity of producing before it the facts upon which we found our respective opinions." This indeed I regarded as an essential condition of the inquiry. I then went on to say, "If the Commission proposes to limit itself to reporting upon this mere question of fact I will freely submit to its decision. If, however, it does not propose to restrict itself, and is empowered to express an opinion upon the interpretation of the fact attested, and on its bearings upon the 'Germ 
Theory of Fermentation' or 'Spontaneous Generation,' then I must respectfully decline to take part in this wider inquiry. . . I desire, therefore, to obtain the assurance of the Commission that no new experiments shall be demanded from either of us, except with the full concurrence of M. Pasteur and myself." After some delay, I was informed by M. Dumas that the Commission also desired, "if possible, only to occupy itself with the experiments of M. Pasteur and myself on the subject of urine treated with potash; and that I had no reason to fear the necessity of a long stay in Paris."

In reply to this I wrote again on July 6 to M. Dumas acknowledging the receipt of his letter (on June 29), but saying: "I do not find in it any distinct acceptance of the conditions mentioned in my letter of May 24, as those upon which alone I should be prepared to repeat my experiments before the Commission, viz. : ( I) the limitation of the report to the question of fact mentioned, and (2) the assurance that no new experiments shall be demanded from either of us except with the full concurrence of both M. Pasteur and myself." On the receipt of a favourable reply I promised to bein Paris on the morning of Saturday, the I 4 th inst. In answer to this $M$. Dumas wrote on the 12 th as follows: "La Commission de l'Académie des Sciences sera dès le I 5 à votre disposition. . . Elle est prête à vous entendre ; mais elle desire, comme vous, que son examen soit borné au point en discussion entre vous et $M$. Pasteur. Ce serait seulement au cas oì vous desiveriez aller phus loin qu'elle aurait à examiner, 
si le temps lui permet d'entraprendre davantage, vôtre séjour étant très court."

I felt it absolutely essential to have a distinct understanding upon these points, because on the mere question of fact I was so positive of being able to reproduce my results, that I cared little as to the constitution of the Commission. But to enter upon the question of interpretation with a Commission so constituted would have been quite a different matter. More than one public comment had been made in this country in regard to the constitution of the Commission. Thus, in one of the annotations of The Lancet for March 10 the following opinions were expressed:-

"This challenge Dr Bastian has accepted, and all who desire, in the interests of science, a solution of the important question involved will watch with more than curiosity the work of the commissioners. In France three members of the Academy have been selected to judge the case. These gentlemen are all well known for the excellence of their labours, but unfortunately hold particular views on the subject they are asked to inquire into. The French Academy's Commission consists of M.Milne Edwards, M. Dumas, and M. Boussingault, all more or less strong supporters of M. Pasteur's view. The objection to such a Commission is obvious; anyone who has worked in science knows how difficult it is to arrive at the truth because of the pernicious influence which preconceived opinions exercise. We would respectfully suggest to the French Academy that there are amongst its members men equally 
trustworthy as the above named, and who have given a fair share of time, trouble, and genius to the settlement of this dispute, and yet who do not accept the views of the germ-theorists. Why not have placed either M. Fremy or M. Trécul on the Commission of the French Academy? It would be well in the interests of science rather than of individuals, if one, at least, of the Commission were not a germtheorist."

An able writer in the Contemporary Review, for April I877, spoke in much the same terms. He said :-

"Those who remember the history of a former French Commission on the very same subject, on which two of the present commissioners served, may be excused for doubting whether the guarantees for a fair and open investigation which were refused to MM. Pouchet, Joly, and Musset, will be conceded to an Englishman who presumes to question the infallibility of M. Pasteur. The three French savants who then disputed Pasteur's views were placed under such restrictions by the course of procedure laid down for them by the commissioners that they considered it due to their dignity to retire. The abortive commission ended, as it is likely this will end, in an ex parte hearing. It is possible, indeed, that MM. Dumas and Milne Edwards may think that more generosity is due to a foreigner who ventures to appear before a tribunal from which every one who inclines to his side of the controversy has been eliminated. If they are disposed to allow of a fair programme, and to concede the guarantee 
of publicity, it is conceivable that an interesting investigation may result; but if it is really desired to obtain a verdict which will carry weight elsewhere than in Paris, it would be well to add to the distinguished savants already nominated some who are less committed to Pasteur's views and less dazzled by the glamour of his great reputation. $\mathrm{W}^{\top} \mathrm{e}$ doubt much whether Pasteur would, even with the strongest guarantees, consent to appear before three English believers in spontaneous generation."

On the afternoon of July I 5 I met the Commission, by arrangement, in the laboratory of M. Pasteur at the École Normale. The Commission was represented only by MM. Dumas and Milne Edwards, M. Boussingault having been compelled to withdraw on account of a recent domestic affliction. M. Pasteur was also present.

The first stage of our discussion was the announcement to me by M. Milne Edwards of his objection to the second condition mentioned in my letter of July 6 , and of his determination to take no part in the inquiry if $\mathrm{I}$ still adhered to this condition. $\mathrm{M}$. Dumas' letter of July I2, in the name of the Commission, and on the faith of which I had come to Paris, was thus at once set aside.

M. Milne Edwards contended that he could not take part in any Academy Commission which had not full power to vary the experiments at discretion; while I, on the other hand, contended that my stay in Paris must, as I had said from the first, be limited to a few days, and that I could not see my way, 
therefore, to consent to the initiation of new experimental conditions. I further urged that the Commission had been appointed to report upon a simple question of fact, that M. Pasteur had challenged me to obtain certain results, before "competent judges," that I had come to Paris to repeat certain well-defined experiments before them, and that they were commissioned to express an opinion thereon and on the experiments of M. Pasteur to the Academy of Sciences.

A very long discussion ensued, but no satisfactory conclusion was arrived at. In the course of this discussion M. Pasteur thought fit to speak of "my errors," and I had to remind him that the Commission had been appointed to decide which of us was in the right.

In the evening I wrote to $M$. Dumas saying that after our conference in the afternoon, "I had a long conversation with $M$. Pasteur, and am going to his laboratory early to-morrow morning to show him the mode in which I make my experiments," adding, "I shall thus be enabled to learn what precise alterations he would desire in order that the experiments may be conducted in a manner satisfactory to himself. Afterwards I trust it may be more possible for me to meet the wishes of the Commission in regard to the inquiry."

At an interview with M. Dumas on Monday, July i6, I proposed a kind of compromise. The proposition was that on the present occasion we should have "the first element" of the inquiry as defined by M. Dumas in his letter of April 25, viz., 
that the opportunity should be given to M. Pasteur and myself of repeating (without variation) the actual experiments upon which we based our respective opinions; that I should then return to London, and after the Commission had expressed its opinion to M. Pasteur and to myself as to any variation in the experimental conditions which they might desire to institute, that I should return to Paris to witness and to perform such modified experiments. The names of MM. Fremy, Trécul, Robin, and Wurtz had been mentioned by me as persons one or other of whom I should like to be placed on the Commission in succession to M. Boussingault. But, at the meeting of the Academy that afternoon, it was announced that $M$. van Tieghem had been nominated to succeed M. Boussingault. This gentleman being a former pupil and present colleague of M. Pasteur, the Commission was still left without a single member who could be considered as representing my views, or even as holding a neutral position between me and M. Pasteur.

The next day I received a note from M. van Tieghem informing me that "the Commission of the Academy would meet to-morrow, Wednesday, at 8 A.M., in the laboratory of M. Pasteur," and asking me to be present in order to commence with the experiments under discussion.

I made all the necessary arrangements that afternoon in M. Pasteur's laboratory for the performance of my experiments, and the next morning at eight o'clock M. Pasteur and I were at the appointed 
place. M. van Tieghem was also there; and shortly afterwards M. Milne Edwards arrived. He, apparently, had again had no communication with M. Dumas since the time of my interview with him; and when told, in reply to a question of his, of the proposition which I had made to M. Dumas, M. Milne Edwards very hastily expressed his disapproval of it, and at once, without listening further, left the laboratory. He was followed by $M$. van Tieghem. I remained, and after one hour M. van Tieghem returned. He informed me that, having waited in vain for the arrival of M. Dumas, M. Milne Edwards had at last gone away.

I stayed in conversation with M. van Tieghem for nearly an hour, in an upper room of M. Pasteur's laboratory. When we came down, much to my surprise, we learned from M. Pasteur that M. Dumas had arrived, that he had been told of the departure of M. Milne Edwards, and that he also had then left, saying that the Commission was at an end-but without in any way communicating either with his colleague, M. van Tieghem, or with myself.

Thus began and ended the proceedings of this remarkable Commission of the French Academy. 


\section{CHAPTER XVI}

FINAL EXPERIMENTS ON THE CAUSE OF THE FERTILITY OF BOILED NEUTRAL OR SLIGHTLY ALKALINE ORGANIC SOLUTIONS

THE reason of the great interest aroused, both in 1 this country and in France, by the urine and liquor potassæ experiments was due to the fact that it was regarded by most people as an absolutely crucial experiment, seeing that all investigators hitherto had found boiled acid urine to remain a barren fluid, and that they were all equally unanimous in believing that the barrenness was due to the fact that all Bacteria and their germs were killed therein by a brief exposure to $100^{\circ} \mathrm{C}$. Surprise was great, therefore, when, at this juncture, the principal opponent of Pasteur's germ theory said he could, with ease, cause life to appear, and apparently by mere physico-chemical processes, in this fluid hitherto universally believed to be germless.

I have already shown how Sir William Roberts and Professor Tyndall, purporting to repeat my experiment, departed widely from my method (did, in fact, what I had specially warned them against doing), and as a consequence utterly failed to obtain my results--by reason of adding an excessive 
amount of potash, when, at last, they broke their potash tubes.

We now have to turn to Pasteur's method of repeating this experiment, and must try to ascertain why he also failed to obtain my results. The actual cause for some little time proved to be a great puzzle to me, though from the first, as will have been seen from the discussion between us, as recorded in the last chapter, I conjectured that his uniformly negative results must have been due, as with the experimenters above referred to, to the fact of his having added potash in excess.

Some months later I made experiments which soon began to throw a flood of light upon the precise cause of his failure, as well as that of others, in producing such results as I had obtained with boiled urine and boiled liquor potassæ.

I chanced to look one day at the fifth edition of Fownes' "Manual of Chemistry," published in I 854 , a book which I had used as a medical student, and on p. 537 found the following statement concerning urea, "It is not decomposed in the cold by alkalies or by the hydrate of lime, but at a boiling-heat it emits ammonia and forms a carbonate of the base." And on the next page there is the statement that " if urine in a recent state be long boiled it gives off ammonia and carbonic acid from the same source."

This fact, one must suppose, was unknown to M. Pasteur, as in his memoir of I862, in reference to a little chaplet-like organism common in unboiled fermenting urine, he said (loc. cit., p. 52): "Je suis 
trèsporté à croire que cette production constitue un ferment organisé, et qu'il n'y a jamais transformation de l'urée ên carbonate d'ammoniaque, sans la présence et la développernent de ce petit végétal." Still, he said, his experiments on the subject were not quite finished, so that "je dois mettre quelque réserve dans mon opinion." As I have shown, however, in a previous chapter (p. I 36), the little chaplet-like organism never appears in any experiments with urine and potash; the urine under these conditions ferments with the development of myriads of short or long Bacilli.

Again, on the same page, M. Pasteur speaking of his experiments in which acid urine had been boiled for a few minutes in a vessel to which calcined air was admitted before the vessel was sealed, said that if it were subsequently exposed in a stove to the influence of a temperature of $25^{\circ}$ to $30^{\circ} \mathrm{C}$. $\left(77^{\circ}\right.$ $86^{\circ}$ F.), "il peut y séjourner indéfiniment, sans éprouvé d'autre altération qu'une oxydation lente de la matière albumineuse de l'urine . . . la limpidité de l'urine reste parfaite, même après dix-huit mois, et il n'y apparait pas la plus petite, production animale ou végétale: elle conserve egalement son acidité et son odeur première." This latter statement, which I have italicised, is absolutely erroneous, as I shall presently show.

Pasteur subsequently became acquainted with the fact which I have quoted from Fownes, and in one of his replies to me (that of January 8), he said in a note: "It is not useless to say here that, contrary to what is generally admitted, urea in aqueous solu- 
tion or in urine is decomposed at $100^{\circ} \mathrm{C}$, and even at temperatures much lower. The product of decomposition is carbonate of ammonia." What I had learned from Fownes, together with this statement, subsequently induced me to give special attention to the subject; and the result has been the full confirmation of Pasteur's recent, in opposition to his earlier, impressions and statements. I doubt, indeed, whether even he was aware of anything like the extent to which such changes will go on in the incubatorand, as we have seen, they were absolutely unknown to Sir William Roberts and Professor Tyndall. The following paragraphs are reproduced from my memoir in the Journal of the Linnean Society' (pp. $38,39):-$

"If a piece of moistened litmus paper is exposed to the steam coming through the capillary orifice of a retort in which ordinary acid urine is being boiled, this paper is for a time rendered faintly blue, showing that some ammonia is being given off, sufficient to make the steam from acid urine faintly alkaline."

"I then proceeded to make some quantitative determinations as to the amount of diminution of acidity occasioned by boiling urine both in an open vessel and, under pressure, in a closed vessel-also as to the subsequent rate of change when urine was kept at different incubating temperatures. Accidents happened to some members of the first series which I prepared with the view of throwing light upon this subject. The results ascertained from 
three of the vessels which escaped were, however, sufficiently significant to show the importance of the inquiry :-

\section{Treatment.}

Result.

I. One fluid-ounce of the $\mathrm{i}$. Fluid found to have acid urine was boiled in a been diminished by $5 \mathrm{iij}$. retort with a capillary neck Acidity of remainder neufor $5^{\prime}$ over the flame pretty tralised by $\mathrm{v} \frac{1}{2}$ minims of briskly, but without spurting- liquor potassa.

Urine whose aciaway of fluid.

dity was exactly neutralised by viii $\frac{1}{2}$ minims of liquor potassae to the

2. One fluid-ounce of same boiled gently over flame $z^{\prime}$, and in can of water, after sealing, for $18^{\prime}$.

3. One fluid-ounce of same 3. Fluid diminished by $5 \frac{1}{4}$ boiled gently over flame $2^{\prime}$, Acidity of remainder neutra. and in can of water, after lised by iv minims of liquor sealing, for $8^{\prime}$. Then placed potasse.

in incubator at $122^{\circ} \mathrm{F}$. for 6 days.

"These estimations, confirmed as they have been by others, soon let in a flood of light. The great diminution of acidity caused by brisk boiling in an open vessel with a capillary orifice was remarkable, and is doubtless principally attributable to the fact that under these conditions the temperature of the fluid is easily raised three or four degrees of the Centigrade scale above the boiling-point ${ }^{1}$; the loss of acidity involved in the diminution of the fluid itself by the mere process of boiling, but without appreciable spurting, was probably small. But when both these conditions are obviated as much as possible by gentle boiling over the flame for two minutes only, and by continuing the exposure to heat, after the vessel has been sealed, in a can of boiling water at a definite temperature of $2 \mathrm{I} 2^{\circ} \mathrm{F}$., the total period of heating may be four times as long without causing

1 See, on this subject, p. I 12. 
as much diminution of acidity as was found in the five minutes of brisk boiling over the flame. Then, again, it appears from the third experiment that the transformation of urea into carbonate of ammonia goes on at a very appreciable rate while the urine is exposed in the incubator to a temperature of I $22^{\circ} \mathrm{F}$. $\left(50^{\circ} \mathrm{C}\right.$. $)$.

"Experiments made with another specimen of urine of very nearly the same acidity yielded the following results. Exactly one fluid-ounce was used, as before, in each experiment.

Treatment.

I. Boiled gently for $5^{\prime}$ over flame, without spurting.

Urine whoseacidity was exactly neutralised by ix 3. Boiled for $2^{\prime}$ over flame, potassie to the for three days.

fluid-ounce.
4. Boiled for $2^{\prime}$ over flame, $1 S^{\prime}$ in can.

5. Boiled for 2 ' over flame, $38^{\prime}$ in can.
Result.

I. Diminished by 5 i.

Acidity $=\mathrm{m}$. vj $\frac{1}{2}$ of liq. pot.

2. Diminished about $5 \frac{1}{4}$. Acidity $=\mathrm{m}$. vj of liq. pot.

3. Diminished by $5 \frac{1}{4}$.

Acidity $=\mathrm{m}$. iv of liq. pot.

4. Diminished by 5 禾. Acidity $=\mathrm{m}$. iv $\frac{1}{2}$ liq. pot.

5. Diminished by 51 .

Acidity $=\mathrm{m}$. iij liq. pot.

The urea in this specimen seemed to undergo change rather more rapidly than in the last, as a careful comparison of the figures will indicate. Experiments 2, 4, 5 also seemed to show that the change takes place most rapidly at first, and subsequently tends to diminish; thus in No. 2, after ten minutes' boiling, over flame and in can, we get a diminution of acidity equivalent to $\mathrm{m}$. iij of liquor potassæ; in No. 4, with an extra ten minutes' boiling in the can the further diminution of acidity only equals $\mathrm{m}$. $\mathrm{i} \frac{1}{2}$ of liquor potassæ; whilst in No. 5, with a still further period of 20 minutes' boiling, the 
additional diminution of acidity was also equivalent to $\mathrm{m}$. $\mathrm{i} \frac{1}{2}$ of liquor potassa.

"Other experiments were made to ascertain whether the change of urea into carbonate of ammonia would still go on at lower incubating temperatures; and this time the urine was one of much higher acidity and specific gravity. Exactly one fluid-ounce was used, as before, in each experiment.

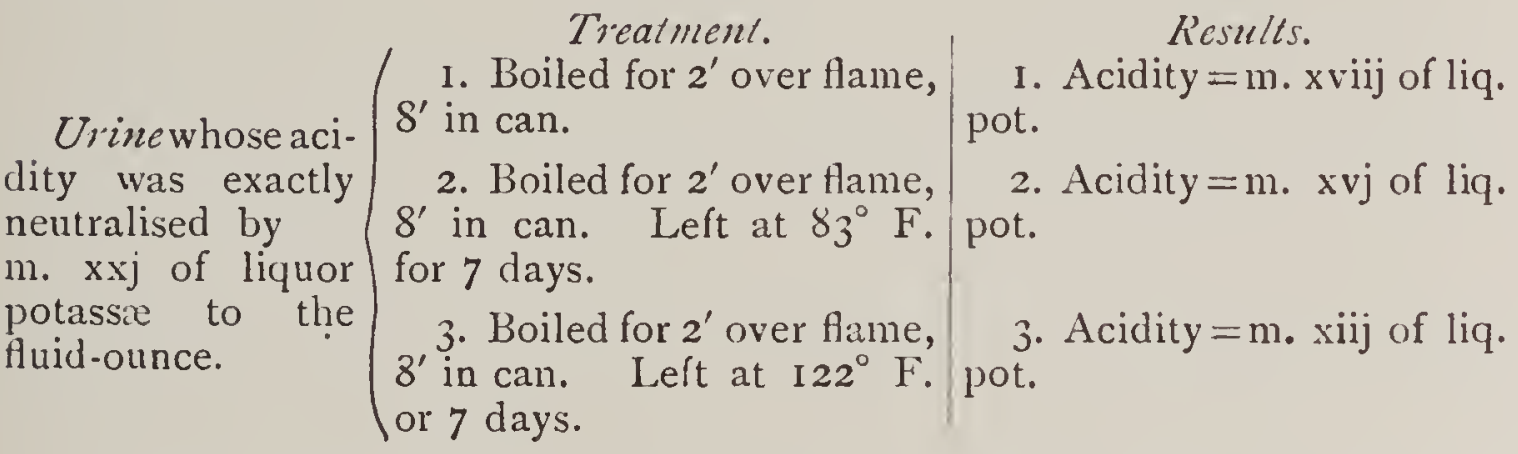

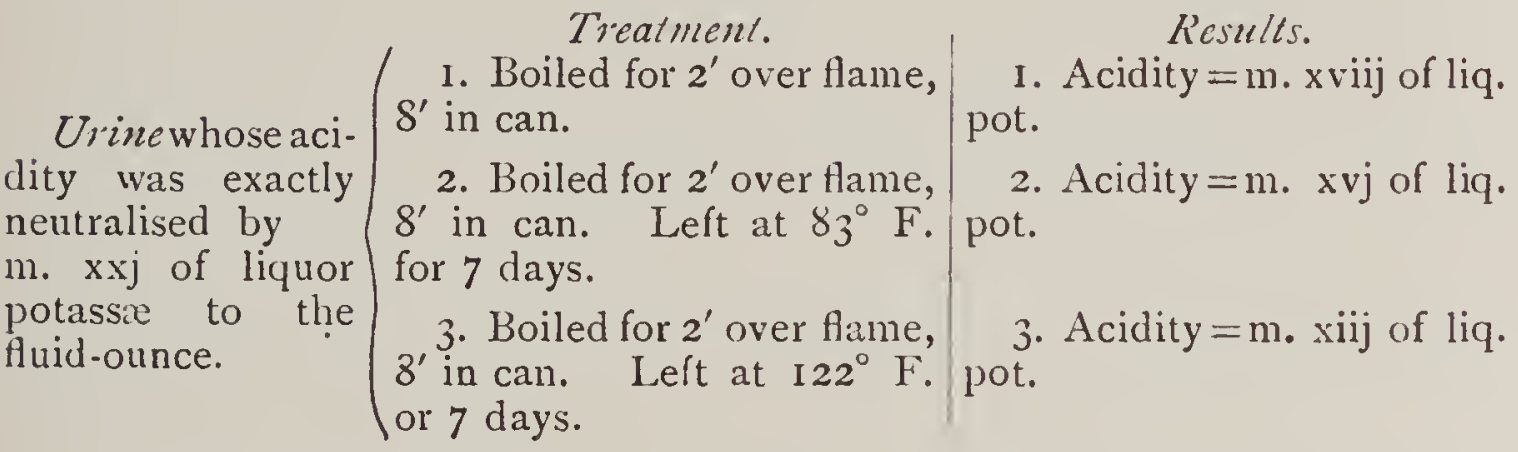

"From this it appears that the diminution of acidity goes on at a very appreciable rate, even at the comparatively low temperature of $83^{\circ} \mathrm{F} .{ }^{1}$ Yet M. Pasteur said (see p. 169) that no alteration in acidity would be found even after an exposure to eighteen months at that temperature!"

We may turn now to the question of the exact mode in which M. Pasteur attempted to reproduce my experiment with boiled urine and super-heated liquor potassæ.

It will be noted from what M. Dumas said to me in his letter of May 5 (p. 159), that the Commission had allowed M. Pasteur to perform his experiments

1 The rate of change is distinctly greater with the higher incubating temperature of $122^{\circ} \mathrm{F}$, as I have shown by another table (loc. cit., p. 40). 
before them in my absence, which, I think, was a wrong course. We each ought to have had an opportunity of seeing the other's experiments performed before the Commission; and, evidently, the intention was that M. Pasteur should be present during the performance of mine.

As the Commission proved an abortive one, on the following Monday (July 23, I877) M. Pasteur submitted a Note on the subject of his experiment to the Academy. It concerned the actual experiment which he had performed before the Commission, and is to be found in the Comptes Rendus for the date mentioned. The experiment is a very ingenious one, and but for one fatal defect, which I shall presently indicate, ought to have led to a successful reproduction of my results.

I will first describe the details of the experiment, and we can then look to the question of interpretation.

Some urine contained in a purified vessel is boiled for ten minutes, and its degree of acidity is tested when it has cooled.

A solution of potash sufficient for the neutralisation of 15 cubic centimetres (half an ounce) of the urine whose acidity has been tested is to be introduced into one branch (A) of the double fambe tube, plugged with cotton-wool, such as is shown in the figure.

The tube is to be hermetically sealed above the cotton-wool plug, and the two narrow prolongations being already sealed, the closed tube containing the solution of potash is placed in a chloride of calcium bath and heated to $110^{\circ} \mathrm{C}$. for ten minutes. Then, 
after being allowed to cool, the chloride of calcium is washed from its exterior.

The tube and the solution of potash, thus purified by heat, is next to have its top cut off above the plug of cotton-wool, and I 7 or I 8 cubic centimetres of the boiled urine are to be aspirated into the side of the tube (B) not containing the potash.

The next directions are these :Plunge the tube into boiling water and heat it to $100^{\circ} C$. for ten minutes. Allow the tube to cool.

Cause 15 centimetres of the urine to pass into the branch $(\mathrm{A})$ containing the potash. Thus 2 to 3 cubic centimetres of the doubly boiled urine will remain in $B$, in order to serve as a control, and show that the urine has been thoroughly sterilised by the tem-

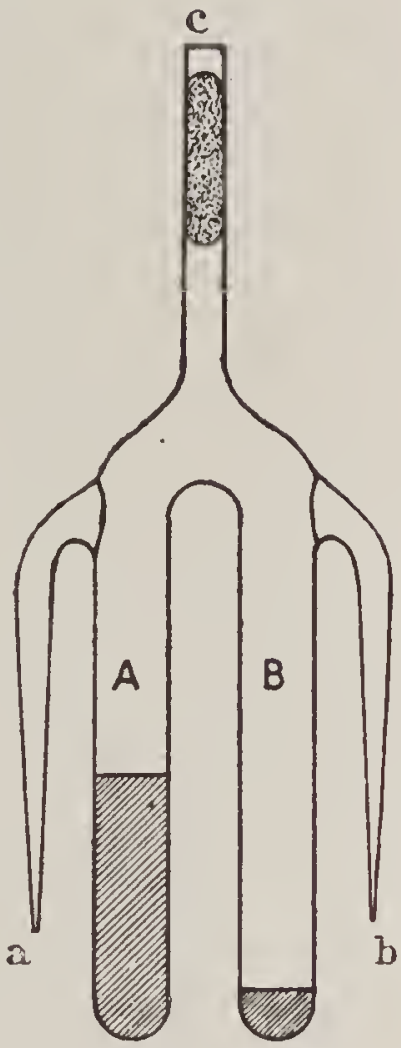

FIG. 8 .

Pasteur's double tube. perature to which it has been exposed, which, Pasteur repeats, is always the case when dealing with an acid urine.

Place the tube in a stove at $50^{\circ} \mathrm{C}$.

Result: Never an appearance of micro-organisms.

M. Pasteur repeated to me in his laboratory, in the presence of M. Chamberland, and even wrote the statement on a piece of paper with a blue pencil, that, operating as I did, organisms were "le plus souvent" to be found in the fluids. He had, in fact, twice said the same thing, in his replies to the Notes 
addressed by me to the Academy (see p. I 54). But in regard to his own experiment he says, "En opérant comme je vais le dire, l'expérience réussit cent fois sur cent, mille fois sur mille, c'est à dire que jamais elle ne donne des bactéries." This boastful statement I can readily believe, simply because it is easy invariably to obtain negative results when a wrong method is employed, though it is not always possible to obtain invariably uniform results, even with a right method, when dealing with a fluid naturally subject to more or less variation.

According to his own statements, M. Pasteur fell into the same error as that which caused Sir William Roberts and Professor Tyndall invariably to find their fluids barren: he also added potash in excess. It is easy to make this plain.

He began by providing a solution of potash sufficient to neutralise I 5 cubic centimetres of urine which had been boiled for ten minutes in an open vessel, instead of an amount distinctly short of full neutralisation, as I had advised; but he did not add the potash till he had boiled the urine again for another 10 minutes, and this time in a vessel closed with a plug of cotton-wool, which, as I have shown (p. I I 2 ), would raise the boiling-point at least $2^{\circ}$ or $3^{\circ} \mathrm{C}$., and thus cause a very distinct lowering of the acidity of the urine.

The extent of the lowering of acidity thus produced may be judged from the extent of lowering shown on p. 17 I to have been produced by boiling an ounce of urine in a retort with a capillary neck for 5 minutes only. The inevitable result of Pasteur's method would have been so to diminish the acidity of the 
urine as to cause the potash to be in distinct excess when added thereto-a cause of failure which, all along, I had suggested to him.

Now let us turn to Pasteur's own interpretation of what he considered to be his success and my failure. It will be borne in mind that in the discussion which occurred between us, before the appointment of the Commission, he invariably attributed my results to fertilisation of the boiled urine (which he still believed to be germless) by the addition of boiled liquor potassa. The living germs were there, as he intimated over and over again. And when he twice declared that, operating as I did, it was a fact that organisms were most frequently to be found in the fluids, it looks as if he said so from sheer confidence in the conclusions he had arrived at in 1862. Thus, even boiled liquor potassæ was, to him, only like one of the fluids he had dealt with at that time, and, as an alkaline fluid, had allowed germs to survive therein at a heat of $100^{\circ} \mathrm{C}$. A truly amazing supposition.

But now, in his final proof of "my errors," as performed before the Commission and published in the Comptes Rendus, not a word is said in favour of this interpretation. A totally new point of view is adopted. He says there were three possible causes of error in my experiment: (I) not using a sufficiently acid urine; (2) not heating the potash sufficiently; and (3) not having purified the vessels employed, by previously super-heating them. He said he was then satisfied that the first two causes 
of error had been "completely eliminated by me," but not the third, since I had not been accustomed to flambe the vessels employed.

Now it is perfectly certain that this is a condition never mentioned and never adopted by Pasteur himself in his previous experiments, as described in I862. In the long interval that elapsed between his reply to me on August 7, and the resumption of the discussion in the following January, he had, during part of this time, been engaged in an investigation of the relative purity of different waters, in conjunction with M. Joubert; an investigation which, as he says in a report of its results in the Comptes Rendus for January 29, was undertaken in consequence of the discussion with me. In a note to this communication, he refers to investigations on the same subject made by Burdon Sanderson in 1871 , who also found Bacteria widely distributed in ordinary tap waters in London, but disagreed with Pasteur as to the frequency of germs of Bacteria in the air-notwithstanding all that Pasteur had said in his memoir of 1862.

Sanderson, therefore, inclined to the conclusion that the Bacteria which appear in experiments relative to spontaneous generation come, as Pasteur puts it, "Exclusivement de l'eau ayant servi au nettoyage des vases, quand on na les flambe pas." Although Pasteur professed not to like these conclusions of Sanderson, the latter point seems to have made a deep impression upon him, so that he soon took a totally different view concerning my experiments. He gave up the interpretation 
that I imported germs into the experimental fluids in the boiled liquor potassæ, and adopted the view that the germs on the walls of a flask were not really killed by contact with boiling acid fluids, so that I (in my capacity of physician) had succeeded in restoring animation to germs which he and all other experimenters had hitherto believed to have been hopelessly killed-all because they had previously never taken the precaution to flambé the vessels made use of in their experiments. ${ }^{1} \mathrm{He}$, in fact, then said explicitly that the germs on the walls of the vessel had not actually been killed in the boiled acid fluids, though their powers of multiplication had been destroyed. This view was subsequently set forth at length by Pasteur's former assistant, Ch. Chamberland (now sub-director of the Pasteur Institute), in a Thesis which he presented in 1879 for his degree of Doctor of Science. Speaking of boiled acid fluids, he says (loc. cit. p. 89), "Mais ils ne sont pas stériles au vrai sens du mot,

1 The method now employed for this purpose was attained by placing the vessels, plugged with cotton wool, in a gas-oven for a time, at a temperature of $150^{\circ}-200^{\circ} \mathrm{C}$. - that is, till the cotton wool showed a faint brown tinge. That this had not previously been deemed necessary is shown by what Chamberland, Pasteur's assistant at the time, said in his Thesis (loc. cit. 1879, p. 21). Speaking of some experiments made about i 876 , he says: "Au moment où j'ai fait ces expériences, je pensait qu'il suffirait de faire bouillir de l'eau pendant quelques minutes dans un appareil pour le priver complétement de germes; mais j'ai découvert depuis, comme je le montrerai plus hoin, qu'il existe certains organismes dont les germes ou spores peuvent resister pendant plus d'un heure à la température de l'eau bouillante." It was after this that he and M. Pasteur introduced the new procedure, and insisted upon the necessity that vessels used in such experiments should be "flambés." 
car ils peuvent encore renfermer des germes vivants qui se développent dans les liquides neutres."

I have given my interpretation of Pasteur's failure in the repetition of my experiment, and it is of such a kind as fully to account for his invariably obtaining negative results. It remains, therefore, for me now to adduce the various kinds of evidence which strongly negative the interpretation he has given of the results obtained by me. They are as follows :-

I. Pasteur's explanation of my positive results may be disproved by direct experiment in the following manner, as I pointed out in my paper in the Journal of the Limean Society (p. 45).

We may take some turbid urine having a neutral or faintly alkaline reaction, in which both Bacteria and Bacilli are swarming and rapidly multiplying, and we may compare the relative effects of heating these organisms, with their reproductive particles, to $2 \mathrm{I} 2^{\circ} \mathrm{F}$. in a neutral and in an acid medium respectively. Three series of experiments are needed :-

\section{Ferment-organisms heated to $212^{\circ}$ F. in an Acid Fluid.}

Add one minim of the faintly alkaline turbid fluid containing fermentorganisms and their germs to one ounce of urine whose acidity is more than equivalent to $m x$ of liquor potassre.

Boil $2^{\prime}$ over the flame, sea!, and then $S^{\prime}$ in boiling water with the flask or retort in an inverted position.

When cool place in incubator at $122^{\circ} \mathrm{F}$.

Kesult. - No change in fluid.
2. Ferment-organisms heated to $2 \mathrm{I} 2^{\circ} \mathrm{F}$. in a faintly Alkaline Fluid.

Place one minim of the same faintly alkaline turbid fluid in a little tube, draw out its neck and seal.

Immerse this tube in a vessel containing one ounce of the same specimen of urine.

Boil $2^{\prime}$, seal, boil $S^{\prime}$ in same man. ner, and when the fluid is cool, break the tube so as to liberate organisms which have been heated in their owen faintly alkaline medium.

Place in incubator with others at $122^{\circ} \mathrm{F}$.

Resul\%.-No change in fluid. 


\section{Control Experiment.}

Boil an ounce of the same acid urine in a small flask, whose neck is plugged with cotton-wool, for $10^{\prime}$.

When fluid has cooled remove cotton-wool plug for an instant, and add one minim of the same turbid fluid, but not previously heated. Quickly replace cotton plug and transfer to incubator at $122^{\circ} \mathrm{F}$.

Result.-Well-marked turbidity and swarms of Bacilli in 18-24 hours.

Such experiments have invariably given the same results. Twelve trials were made with a urine of I $30 \mathrm{sp}$. gr., whose acidity was equal to Io minims of liquor potassæ per ounce; and nine trials were made with a urine whose acidity equalled 25 minims per ounce, and whose sp. gr. was IOzO.

These experiments are of much interest, because they show in a most decisive manner that the mere neutrality or slight alkalinity of the medium in which the ferment-organisms are heated is quite unable to preserve them from the destructive influence of a temperature of $100^{\circ} \mathrm{C}$. They show also that Bacilli, not previously boiled, develop and multiply with great freedom even in a very highly acid urine.

2. But the proof is also abundant and absolute that Bacilli and their germs are capable of developing and multiplying after they have been boiled in acid fluids. I need only refer to my experiments with simple unaltered acid turnip infusion in proof of this-experiments which have been confirmed by Burdon Sanderson as well as by Professor Huizinga. Taken at their lowest value, such as my opponents favour, these experiments are a direct disproof of the explanation given by Pasteur, and later by Chamberland, of my urine and liquor 
potassæ experiments. Abundant evidence of the same kind will be found in later Chapters (xix. and $\mathrm{xx}$. ) in which the results of my new experiments with superheated saline solutions are detailed.

This is all that is needed for the direct disproof of Pasteur's interpretation of my experiment, namely, that the acid urine was not really sterilised by boiling; that it contained germs, previously supposed to be dead, because such germs after boiling were no longer able to develop and multiply in the acid fluid.

There are, however, a few collateral points remaining over from this discussion concerning which some words ought to be said. They are these : (a) As to the assumed necessity for the experimental vessel being superheated; $(b)$ whether ordinary liquor potassæ is a germ-containing medium; $(c)$ whether healthy urine, fresh from the bladder, guarded from contamination and in fambé vessels, can be made to ferment; and $(d)$ the effects of adding liquor potassæ in different proportions to such urine.

\section{a. Concerning the assumed necessity for Super- heating the Experimental Vessels.}

Although, as I have said, there is no evidence that Pasteur in his previous experiments (as recorded in I 862), ever considered it necessary that the vases should be flambes, this precaution was considered desirable by Burdon Sanderson in 1873 , and in the experiments which I performed with him in that year, two of the third series (p. 105) were made 
with calcined retorts, and yielded just the same kind of results as were obtained in other experiments in which no such precaution was taken. It cannot be said, moreover, that in these experiments the fertility was really due to the turnip infusion containing minute particles of cheese, since, as Professor Huizinga showed, soluble peptones may take the place of cheese without any alteration in the experimental results.

Still, in all the experiments subsequently to be referred to (except where, as we shall see, this procedure was purposely omitted in the first set to which reference will be made) I have had recourse to this additional precaution, and the walls of the vessels have been thoroughly fambés before they have been used. Yet all that Pasteur could say pointing to such a necessity, as a result of his examination of different waters made in concert with M. Joubert, was that the ordinary water of a laboratory (tap water), with which experimental vessels are washed, contains Bacteria and their germs; and that vessels which were allowed subsequently to drip and dry, might or would contain on their walls some germs able to withstand a temperature of " $100^{\circ} \mathrm{C}$., at least, when in the moist state . . during several minutes" (Comptes Rendus, July 23, 1 877). This, indeed, was now supposed by him to be the source of the germs, which, after they had been boiled, were incapable of developing in an acid medium ; and such germs were further supposed by him to be spores of Bacilli.

How little this view is in accord with direct ex- 
periments destined to test the contaminating effect of boiled tap-water may be gathered from the following observations :-

In 1883 , when making other experiments of an allied nature, I was anxious to ascertain what would be the result of simply boiling tap water in ordinary flasks previously cleaned therewith, before adding pure urine and then exposing the vessels to a rather high incubating temperature.

Urine of medium acidity was passed with all proper precautions (as dictated by Pasteur) into one of the flambé receivers used by Lister, and immediately replugged.

Three flasks were two-thirds filled with tap water, and the fluid in A was boiled for $5^{\prime}$, in $\mathrm{B}$ for $\mathrm{IO}^{\prime}$, and in $\mathrm{C}$ for $\mathrm{I} 5^{\prime}$, so as to imitate the ordinary conditions to which the walls of experimental vessels had previously been subjected. When the contents of each flask had been boiled for the time indicated the boiling water was poured out, some pure urine from the receiver was quickly introduced, and each flask (having been plugged with carbolised cotton wool) was put into an incubator at a temperature of about I I $3^{\circ} \mathrm{F}$.

The results were as follows: $\mathrm{A}$ in $3^{\frac{3}{4}}$ days became turbid, and was found to contain Micrococci only; $\mathrm{B}$ became turbid in $3 \frac{1}{2}$ days, and contained Micrococci only; while $C$ became turbid in $2 \frac{1}{2}$ days and also contained Micrococci only.

These results surprised me much, first at finding Micrococci only, instead of Bacilli, and secondly, at finding that the fluid in the flask in which water 
was boiled the longest became turbid the most rapidly.

Again, I tried this experiment with another specimen of urine (requiring eleven minims of liquor potassæ for the neutralisation of one ounce) which was collected with every precaution in a flambe receiver and passed into two flasks in which tap water had been boiled for Io'. They were placed in an incubator at I I $2^{\circ} \mathrm{F}$., when one of them became turbid in twenty-four and the other in thirtysix hours, and on examination each fluid was again found to contain Micrococci only.

Two other flasks were treated in the same way, and some of the same stock of urine was added after the IO' boiling, but now super-heated liquor potassæ sufficient for neutralisation was added to each flask before it was plugged and placed in the incubator. The result was that both became turbid in twenty hours, and in each flask there were found multitudes of Micrococci, together with Streptococci and Bacilli of all sizes up to long Leptothrix-like threads.

In three more experiments with a different urine, in which tap water was boiled in the flasks for ten minutes before the pure urine was added (without neutralisation), Micrococci only, again appeared in 2 I to 36 hours.

From Pasteur's point of view these results were very remarkable. The fluids, in order to accord with his interpretation of my experiments, ought in each case to have yielded Bacilli only, and neither he nor any one else has ever attempted to show that 
Micrococci can survive an exposure in fluids to $100^{\circ} \mathrm{C}$. Yet micrococci alone always appeared.

\section{(b) Is ordinary unboiled liquor potassa a germ- containing medium?"}

That liquor potassæ when boiled is not, as Pasteur at first contended, a germ-containing medium, is obvious from the fact which I mentioned to him, that when in too small a quantity, and even when in too large a quantity, it would not fertilise boiled urine on addition thereto. He did not venture to attempt any proof of his contention. He might easily have recognised his error in regard to this, by doing what I have since done, in order to show that even liquor potassæ which has not been heated is a germ-free liquid.

I inoculated twelve flambé flasks, plugged with cotton wool, containing sterile urine, which had been in the incubator unchanged for six or seven days, ${ }^{1}$ each with a drop of liquor potasse taken with a flambé pipette from a bottle which had been very frequently opened and exposed to the air. These flasks were placed in the incubator at a temperature of $110^{\circ} \mathrm{F}$, and after twenty-four hours the fluid in one of them was found to be turbid with short Bacilli, that in all the others being clear." At the

1 They were some of the fluids in which I had been testing the death-point of Bacilli spores, as detailed in Chapter $x$.

"Of course, there is alway's a slight risk of accidental contaminations during the removal of a cotton wool plug, to permit of inoculation, and its subsequent reintroduction, though I tried to guard against this, as much as possible, by brushing round the top of the flask between its edge and the plug with a camel hair pencil wet with a zo per cent. solution of carbolic acid. 
end of the fourth day the fluids in the eleven flasks were still quite clear. Using all precautions, each flask was then inoculated with a single drop of urine turbid with Bacilli, with the result that in seven of the eleven Hasks, the fluids became turbid with Bacilli in from 20 to 36 hours. The fluids in the other four flasks remained clear for another three days, and were not kept longer under observation.

(c) Can pure Urine in flambé vessels, and guarded from contamination, be made to ferment?

In his Note of July I7, 1876, M. Pasteur attached little importance to the high incubating temperatures which I have employed; moreover, he said that potash in proper proportions may be added even to unboiled urine, when passed with all proper precautions into a super-heated vessel, without causing it to ferment. And in this Note of January 8, 1877, written in collaboration with $\mathrm{M}$. Joubert, he said the discussion concerns a fact, viz., "whether urine which has been boiled so as to be sterile, and better still fresh urine, unaltered, as it passes from the bladder, will after neutralisation by potash, and at a temperature of $50^{\circ} \mathrm{C}$., produce organisms." Pasteur contended that no fermentation would occur, so long as the potash added was pure.

During the year 1883, when I took up the question of the power of resisting heat possessed by spores of Bacilli which had been in a state of desiccation for over five years, in reply to Professor Tyndall's assumptions, I also performed a very 
large number of experiments with fresh urine, passed into superheated vessels with all the precautions dictated by Pasteur and by Lister. These experiments dealt with two points in particular: first the effects of mere high incubating temperatures upon such a fluid (which we are to consider in this section); and, secondly, the effects of high temperatures when combined with different proportions of liquor potassæ, which will be referred to in the next section.

I found that pure urine, passed with all precautions into a flambé vessel and protected from contamination, would, as Pasteur and Lister have said, remain pure if kept at a low temperature of about $70^{\circ} \mathrm{F}$. $\left(2 \mathrm{I}^{\circ} \mathrm{C}\right)$.

But if, in other pure vessels, some of this urine was put into an incubator maintained at a temperature of I $3^{\circ} \mathrm{F} .\left(45^{\circ} \mathrm{C}\right.$.), I have over and over again found that the urine in such vessels would become turbid within three days, and on examination would be found to contain myriads of Micrococci only.

On the other hand, pure urine in pure vessels if maintained at an incubating temperature of about $116^{\circ} \mathrm{F} . \quad\left(47^{\circ}\right.$ C. $)$ would not yield Micrococci. The fluids would under these conditions, either remain unchanged, or, the acidity of the urine being low, they would become turbid and swarm with Bacilli. For the appearance of micrococci in urines to which no liquor potassæ has been added, the fluids must be exposed to the lower incubating temperature. 
(d) The Effect of adding liquor potassa, in different proportions and with different Incubating Temperatures, to pure urine in flambe vessels.

On very many occasions pure urine passed into a fambé vessel, if fully or two-thirds neutralised with superheated liquor potassæ, and subsequently exposed to temperatures ranging from I $16^{\circ}-122^{\circ} \mathrm{F}$. $\left(44^{\circ}-50^{\circ} \mathrm{C}\right.$.), was found to become turbid more rapidly, that is, in seventeen to twenty-four hours and, on examination, to show no Micrococci but swarms of Bacilli.

On the other hand, if lesser amounts of liquor potassa were used, sufficient for half-neutralisation, or a little less or more, together with incubating temperatures between $117^{\circ}$ and $116^{\circ} \mathrm{F} .\left(44^{\circ}-47^{\circ} \mathrm{C}\right.$. $)$ the fluids would generally yield a mixture of Micrococci, Streptococci, and Staphylococci, together with Bacilli varying much in size.

I thus found that using pure urine in fambe vessels, without or with liquor potassæ in different proportions, and at different incubating temperatures, I could, almost at will, procure Micrococci alone, Bacilli alone, or a mixture of these with Streptococci more especially.

In all the experiments where liquor potassæ was used, I had-in order to eliminate all objections, however futile-previously heated it in tubes to $105^{\circ}-\mathrm{I} 20^{\circ} \mathrm{C}$. for one hour, as I had formerly done to meet unfounded objections made by Pasteur in regard to the original experiments. 


\section{CHAPTER XVII}

WAS PASTEUR RIGHT IN SAYING THAT NEUTRAL, OR SLIGHTLY ALKALINE, GUARDED ORGANIC FIUUIDS PREVIOUSLY EXPOSED TO I IO ${ }^{\circ} \mathrm{C} .\left(230^{\circ} \mathrm{F}.\right)$ ALWAYS REMAIN BARREN?

THERE can be no doubt that many organic fluids 1 which have been heated only a few degrees above the boiling-point, and which are subsequently kept at a temperature no higher than $86^{\circ} \mathrm{F} .\left(30^{\circ} \mathrm{C}\right.$. $)$, will remain barren. ${ }^{1}$

It is well-known that neutral or faintly alkaline fluids will ferment after exposure to this higher temperature better than acid fluids. If, as I have endeavoured to prove, the former fluids have, owing to their constitution, a greater tendency to ferment than acid fluids, this is only what might have been expected. It stands to reason that if heat beyond a certain intensity tends to stifle the fermentable qualities of organic liquids, a lower degree of heat would extinguish these qualities in the less fermentable fluids than would suffice to annul them in the more fermentable fluids. If fermentability is a quality of organic fluids inseparable from, or solely dependent upon, the presence of certain living

1 This chapter is a reproduction, with only slight additions and a few verbal alterations, of one section of my Memoir in the Journal of the Linnean Society (Zool.), vol. xiv., No. 74, 1878. 
organisms, then the fact of fermentability persisting in an organic liquid after it had been heated to $105^{\circ} \mathrm{C}$. $\left(22 \mathrm{I}^{\circ} \mathrm{F}\right.$.), would, of course, have been a proof that living organisms in some form could withstand the influence of such a temperature.

M. Pasteur found that specimens of milk, and of sweetened yeast-water neutralised by carbonate of lime, would, in fact, often ferment after they had been heated to $105^{\circ} \mathrm{C}$.; and he very soon arrived at the conclusion that this was owing to the survival of Bacteria and Vibrio germs in these fluids. Finding, however, that in his hands even such fluids were invariably sterilised after they had been exposed for a few minutes to a temperature of $110^{\circ} \mathrm{C}$. $\left(230^{\circ} \mathrm{F}.\right), \mathrm{M}$. Pasteur proclaimed that such a degree of heat was certainly destructive of all germs in fluids-even in those above mentioned where, as he thought, they most stubbornly resisted the destructive influence of heat.

The experiments already recorded have shown that Pasteur's explanation of the cause of the fermentation of the neutral fluids after boiling and after heating them to $105^{\circ} \mathrm{C}$., is without sufficient foundation in fact-that it is, in short, directly negatived by strict crucial experiments.

I now have to bring forward additional evidence tending to show that the induction of this same distinguished investigator as to the invariable barrenness of neutral liquids after they have been heated to I $10^{\circ} \mathrm{C}$., is also one which is overthrown by a wider experience. By having resort to a simple physical agency (viz., a higher incubating-temperature than 
that which M. Pasteur formerly made use of), it can be easily shown that many properly prepared fluids may be made to ferment after they have been exposed even to I IO ${ }^{\circ} \mathrm{C}$. and upwards.

This final evidence is, of course, not strictly needed for the overthrow of the foundations on which M. Pasteur based his germ-theory; what has already been brought forward concerning the fertility of boiled acid fluids, and the cause of the fertility of boiled neutral fluids, being of itself abundantly sufficient for their overthrow.

This evidence, which I have already given as to the cause of the fertility of boiled neutral fluids, also goes far to undermine the foundations of the belief of other investigators as to the "survival of germs" in any previously boiled fluids. These beliefs all take their origin either from Pasteur's supposed proof of such a phenomenon, or from facts of a similar order to that by which he was supposed to have demonstrated it. The process is essentially this, and it has been often repeated: ist, a deeply rooted conviction that living matter cannot arise de novo; 2nd, the finding of living matter in fluids which have been boiled or further superheated. Such a combination of fact and conviction leads to the facile conclusion that germs have survived the boiling, quite irrespective of the duration of the exposure. And, similarly, the above-mentioned conviction continuing to be firmly rooted, the finding of living organisms in guarded fluids which have been heated to $230^{\circ} \mathrm{F}$. may be immediately explained in the same way: 
"survival of germs" will be again the verdict, in spite of previous statements to the contrary, and independently of all direct evidence. Is fertility attained again, after another alleged death-point has been passed? Do fluids which have been heated to $248^{\circ} \mathrm{F}$. ferment? First the facts are denied; and when these are established, again comes the revocation, without any independent warrant, of previous beliefs, and the old cry "survival of germs."

To those who are wholly inspired by the conviction that a de novo origin of living matter is impossible, the following statements of experimental results will doubtless carry with them no significance, other than that above indicated. Still, for the sake of those who are not so imbued, it will be worth while to cite them. The tubes employed in these experiments were all thoroughly flambes, during their preparation, and were used shortly afterwards.

Urine, almost neutralised with liquor potassæ, which has been heated to $2 \mathrm{I} 2^{\circ} \mathrm{F}$. for one to three hours in closed airless flasks, will not unfrequently ferment in two or three days, when kept at a temperature of $122^{\circ} \mathrm{F}$. A very little more or less of alkali, however, will, with some urines, suffice to prevent the occurrence of this change.

In sealed flasks, half-full of neutralised urine and half-full of ordinary air, which have been heated in a calcium-chloride bath to $230^{\circ} \mathrm{F}$. ( $110^{\circ} \mathrm{C}$.) for $5^{\prime}$ to $3 \mathrm{O}^{\prime}$ fermentation takes place much more rarely. Still I have seen it occur fourteen times out of fifty trialsshowing itself in the course of one to three days. 
In seven of these cases the fluids did not become generally turbid, though one or more tufts of Bacilli appeared in each : these were all specimens of the same urine which had been heated to $230^{\circ} \mathrm{F}$. for 5 minutes. In the other seven instances there was very slight general turbidity, and the vessels had been heated to $230^{\circ} \mathrm{F}$. for 30 minutes.

In twelve trials with faintly acid beer-wort, partly of I060 specific gravity and partly diluted to I030, heated in tubes with air to $230^{\circ} \mathrm{F}$. for Io minutes, fermentation did not once occur; though, as in the experiments with urine, the fluids were, after this superheating, kept for many days at a temperature of $122^{\circ} \mathrm{F}$.

The results with hay-infusion, acid, ${ }^{1}$ neutral, and faintly alkaline, having a specific gravity of about 1005, have been much more successful. In each case about half an ounce of the fluid was used, halffilling a tube which was sealed when cold; so that above the fluid there was ordinary air. After heating the tubes in the calcium bath, some of them were exposed in the incubator to temperatures between $104^{\circ}$ - I $3^{\circ} \mathrm{F} .\left(40^{\circ}-45^{\circ} \mathrm{C}\right.$. $)$, and others to a temperature of $122^{\circ} \mathrm{F}$.

In fourteen cases the infusions were heated to $230^{\circ} \mathrm{F}$. for five minutes; and in every one of these tubes organisms showed themselves within 36 hours. In twenty-one cases they were heated to $248^{\circ} \mathrm{F}$. $\left(120^{\circ} \mathrm{C}\right.$.) for 30 minutes; and in five of these latter trials (all with the same hay-infusion)

${ }^{1}$ Only three times in this state. In the other cases liquor potassæ was added so as to make it neutral or slightly alkaline. 
no fermentation subsequently occurred. In the other sixteen trials more or less distinct fermentation supervened-though in some the signs of change before opening the vessels were only slight. The characters of the organisms presented by these different fluids will be referred to in the next section.

On another occasion, five tubes were charged with a neutralised hay-infusion, and after the tubes were sealed they were heated to $257^{\circ} \mathrm{F}$. (I $25^{\circ} \mathrm{C}$.) for thirty minutes. They were exposed to an incubating temperature of $I 10^{\circ}$ to $\operatorname{II} 5^{\circ} \mathrm{F}$., and on the fourth day the fluids in each were found to be slightly turbid. On examination they showed an abundance of Torulæ mixed with Bacteria.

Six trials with neutralised potato-infusion, having a specific gravity of ror I, yielded no evidence of fermentation. Twice infusions were heated to $248^{\circ} \mathrm{F}$. ( $120^{\circ} \mathrm{C}$.), and four times to $230^{\circ} \mathrm{F}$. ( 1 I $0^{\circ} \mathrm{C}$.) for 30 minutes.

Sixteen trials with a neutral cucumber-infusion, having a specific gravity of 1003 , heated to temperatures varying from $22 \mathrm{I}^{\circ}-248^{\circ} \mathrm{F}$. $\left(105^{\circ}-\mathrm{I} 20^{\circ} \mathrm{C}\right.$.) for 20 minutes were also attended by uniformly negative results.

But in forty experiments with good cow's milk heated in closed tubes half full of air to $230^{\circ} \mathrm{F}$. $\left(110^{\circ} \mathrm{C}\right.$.) for $5-60$ minutes, and also in five other experiments in which the milk was heated to $240^{\circ} \mathrm{F}$. ( 1 I $5{ }^{\circ} 5$ C.) for 10 minutes, fermentation more or less marked occurred in each case in from 2-to days.

The great variation in these results, especially with 
different fluids similarly heated, makes it seem almost impossible to account for them solely by reference to the death-point of germs, as some will doubtless attempt to do. Why should this deathpoint be so different in different fluids?

Then, again, these new experiments, like those I have previously recorded, will be found entirely to contradict Professor Cohn's position, that Bacilli are the only organisms which appear when boiled or superheated fluids ferment. If it is true, as he says, that other organisms are all killed by temperatures below $212^{\circ} \mathrm{F}$. ( $100^{\circ} \mathrm{C}$.), when they are immersed in fluids, how are germ-theorists able consistently to explain the appearance, under such conditions, of swarms of Micrococci, as detailed in the last chapter and now again to be referred to; and of Torulæ, some of which have been enabled to develop typical and well-formed mycelia? The conditions and modes under which these different organisms have appeared in the present series of experiments are now to be described.

Signs of Formentation in the Suporheated Fluids employed in the foregoing Experiments.

Urine.-The turbidity caused by precipitated phosphates will never, by the experienced worker, be confounded with that due to fermentation. He should, indeed, as far as possible, and except for special purposes, avoid dealing with urines which are prone to manifest this phenomenon. When the cloudiness from this cause is very slight, it some- 
times disappears as the urine grows cool; but when it is considerable, a thick white deposit gradually falls, leaving the supernatant fluid quite clear.

All such deposits of phosphates, however, will soon subside after the vessel has been placed in the incubator, so that after twelve hours or less we have to deal with a clear supernatant fluid in which any subsequent turbidity may be easily discriminated. In airless vessels, even the first haziness of the fluid seems to show itself uniformly throughout the liquid, and it is always accompanied by a slight diminution of colour. The urine becomes of an appreciably lighter shade. When the fermentation is vigorous, the haziness of the fluid rapidly passes over to a wellmarked turbidity, which will generally continue for a long time, and without the formation of the slightest scum or pellicle on its surface.

If the fermentation is less vigorous, it may manifest itself in one or other of three ways :-

(a) It may never pass beyond a faint haziness of the fluid, even where the vessel is kept in the incubator for a week or two; ${ }^{1}$ and in such cases the organisms are very scarce, not more than one or two being discoverable in any one field of the microscope. ${ }^{2} \mathrm{~A}$

${ }^{1}$ It is possible for an inexperienced observer to confound this condition with another in which the fluid remains quite unaltered, but in which the glass is attacked and made dim by the fluid. This occurs occasionally when some urines are kept long at a temperature of $122^{\circ}$ F.; and it is especially apt to occur if the temperature should rise a few degrees above this point.

${ }^{2}$ A similar scantiness of organisms is also often met with in the blood of certain animals suffering from splenic fever, though in others they may swarm abundantly. (Quart. Journ. of Micros. Science, Jan. I877, p. 87.) 
change of this kind is also often late in manifesting itself.

(b) The fluid itself may remain perfectly clear; but at the sides of the vessel, or on some phosphatic sediment at the bottom, one, two, three, or more little whitish tufts may show themselves, which continue to increase in size for two or three days, the fluid itself still remaining perfectly clear. These are not tufts of some fungus, as might be thought from their appearance, but are tangled masses of long Bacilli-filaments. ${ }^{1}$

(c) In other cases the fluid itself may remain clear, and no tufts may show themselves; but slowly, and after many days, more or less of a flocculent sediment accumulates at the bottom of the vessel in which organisms are to be found. This latter kind of change has not been much considered in this paper $;^{2}$ it is one which has only shown itself on a few occasions, and is, moreover, one which, judging from my

1 Sometimes they have more the appearance of what Professor Cohn describes as Vibrio serpens ("Beit. zur Biolog. der Pflanz.," Bd. i. Heft 2, Taf. iii. fig. I8). But I entirely disbelieve in the propriety of regarding such differences as he distinguishes between these forms as having any specific or even generic value. Dr Warming, of Copenhagen, says: "Les Bactéries sont douées, en réalité, d'une plcrsticité illimitée, et je crois qu'il faudra renoncer au système de $M$. Cohn et de quelques autres savants qui caractèrisent les genres et les espèces d'après leur forme." Quoted in Qucrt. Journ. of Microsc. Sci., Jan. I877, p. 85. It is, however, only fair to add that Professor Cohn was himself by no means free from doubt on this subject.

2 This is a kind of change which I have spoken of as "smouldering fermentation." In the latter portion of a paper in the Proceedings of the Royal Society', No. I45, I873, I have fully described the different degrees of fermentability apt to be shown by various kinds of fluids when, heated to different degrees, or when, after the heating, they are subsequently exposed to different incubating temperatures. 
previous experience, is more apt to manifest itself when we deal with lower incubating-temperatures, as from $77^{\circ}-96^{\circ} \mathrm{F} .\left(25^{\circ}-35^{\circ} \mathrm{C}\right.$ ), rather than with $122^{\circ} \mathrm{F}$.

The fermentation which takes place in boiled or superheated urine is altogether different from that which occurs in unheated urine in open vessels.

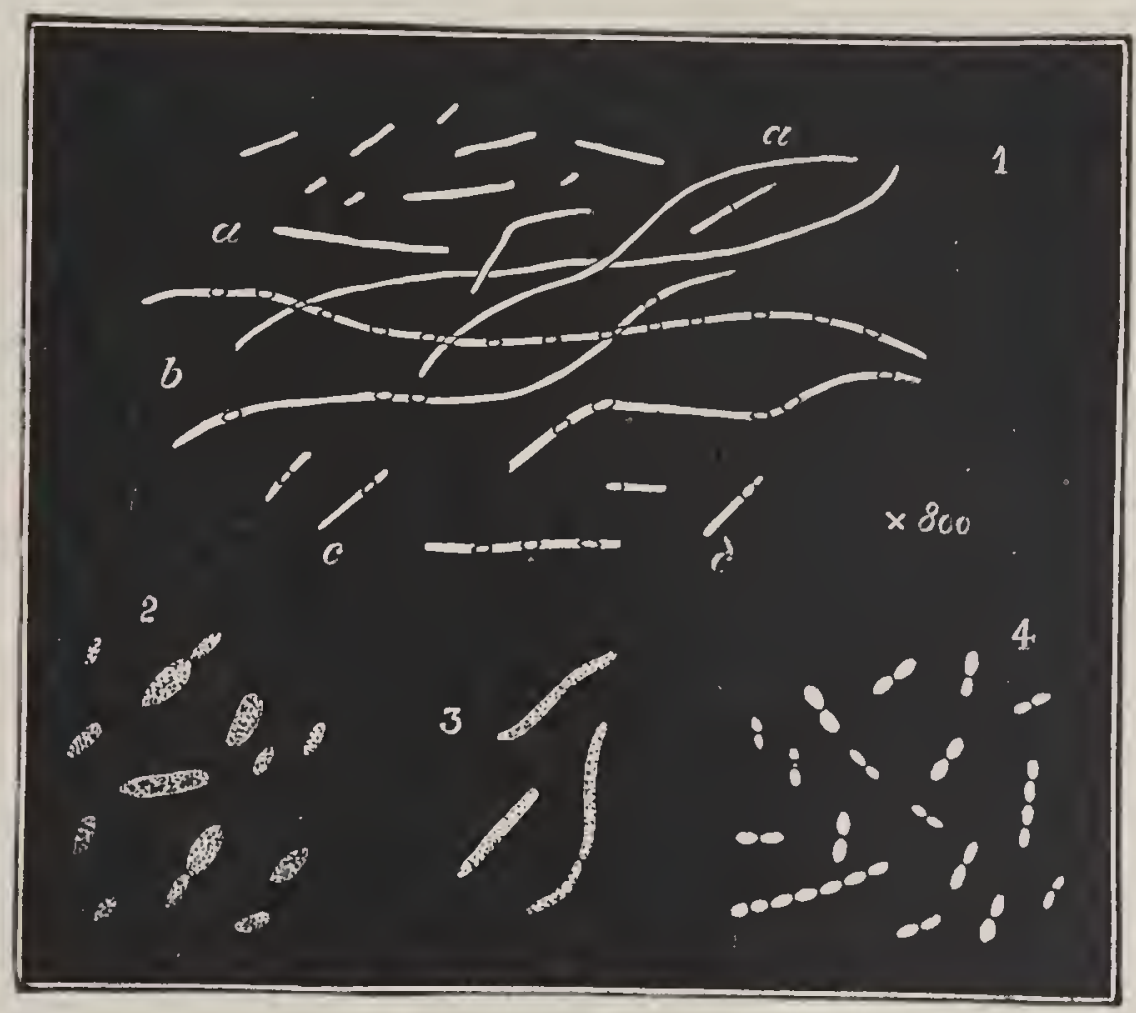

Fig. 9.

Micro-organisms from tubes containing urine or hay-infusion.

I. Urine Bacillus. $a, a$. Short, medium length, and long filaments. $b, b$. Filaments bearing spores. $c, c$. Small fragments of such filaments.

2. Small Torulc from hay-infusion.

3. Vibrio Rugula from hay-infusion.

4. Micrococci in the figure-of-8 form and as short chains (Streptococci), from milk and from hay-infusion.

Even when the fluid has become quite turbid, it is never fotid. The odour may be either quite unaltered in this respect or, at most, there may be a slight increase of its urinous character. ${ }^{1}$ As a rule,

${ }^{1}$ In partly neutralised diabetic urine which has undergone fermentation in an airless vessel, I have on two or three occasions found an extreme fœtidity of the fluid ; and this is, moreover, an extremely common occurrence where turnip-infusion ferments under the same conditions. 
too, the organisms found are Bacilli (Fig. 9, a), either short and unjointed (straight or bent); or longer, and representing what $I$ have hitherto described as Vibriones; or longer still, in the form of unjointed Leptothrix threads. I have all along contended that these were merely different forms of the same organism ${ }^{1}$ and now this is the accepted view, and they are all regarded, in accordance with the nomenclature of Cohn and Eidam, as Bacilli of different lengths.

In airless flasks nothing like spore-formation shows itself in the filaments; so that in this respect the Bacillus of urine agrees with that of hay and of splenic fever. There is a still further agreement; since in open vessels, or in those which are merely plugged with cotton-wool, a scum forms on the surface of boiled urine inoculated with Bacilli in twenty-four hours - when at a temperature of $100^{\circ} \mathrm{F} . \quad\left(38^{\circ}\right.$ C. $)$ - composed, in the main, of filaments which within forty-eight hours will show the highly refractive bodies in their interior (Fig. 9, b), and indeed, partly break up (c) after this formation of "spores." All this agrees with the description which has been given by Cohn and Koch of the hay-Bacillus, and of that of splenic fever. It is quite evident, therefore, that we must recognise the existence of a urine-Bacillus; but I do not on that account attempt to confer upon it any new specific name. Such a procedure would, I think, not only tend to confirm erroneous notions as to the distinctness of the life-history of these

${ }^{1}$ See Nature, July I4, I870, p. 22 I. 
lower forms, but would be utterly useless even from the point of view of the species-makers. One might at once describe as new species, and dignify with new names, the Bacillus of turnip-infusion, that of cucumber, and of fifty other fluids. But would any rational end be attained thereby?

Bacilli, however, are not the only organisms to be met with in boiled or superheated urine. On rare occasions we may meet with other forms. Thus, where oxygen has been added by electrolysis, and where the reaction of the fluid has continued faintly acid, I have on two or three occasions found Bacteria composed of two little ovoid cells-something like $B$. termo, in fact, except that they are always quite motionless. These have been seen in cases where there has been a feeble fermentation of type $(c)$; and in some of my earlier experiments with results of the same kind (though no oxygen was added), and where the incubating-temperature had been $86^{\circ}-95^{\circ} \mathrm{F}$., I have found Diplococci, Streptococci as short chaplets, and small Torulæ. Lastly, in one or two specimens of partly neutralised diabetic urine with which I experimented in the spring, I found, some days after the boiling, Torulæ growing freely in the midst of flakes composed of aggregated Bacilli.

Where the fermentation takes place in superheated vessels which have been sealed whilst the fluids were cold, so as to contain air, after the manner introduced by Spallanzani and Needham, and so often adopted since their time, the process is apt to show itself first by slight turbidity near the surface of the fluid.

Hay-infusion, when treated in the manner last 
mentioned, and when it is heated to $230^{\circ} \mathrm{F}$. ( $110^{\circ}$ C.) or upwards, does not, as a rule, exhibit any very well-marked turbidity. Nevertheless the fluid may grow perceptibly clouded; and while it becomes appreciably lighter in colour, a flocculent precipitate gradually accumulates. In other cases we may have whitish tufts of organisms manifesting themselves, and visibly increasing in size, while the fluid around remains clear. Lastly, in the least satisfactory cases, we may have neither of the foregoing signs of change, but only a slow accumulation of a sedimentary matter, amongst which, in certain cases, organisms are to be found that are unquestionably living. Still, it is also true that deposits of mere amorphous and crystalline matter will generally accumulate after a time at the bottom of even a well-filtered hay-infusion, in cases in which no fermentation is initiated. Hence it is that this latter kind of change is unsatisfactory, and we need the microscope to tell us whether or not we really have to do with a "smouldering fermentation" in which a limited number of organisms are present.

It will thus be seen that the fermentation is almost always less vigorous in these superheated hay-infusions than where they are merely boiled, and its modes of manifestation are almost exactly the same as for urine. I have, however, met with some exceptions to this. Thus, last summer fourteen specimens of a hay-infusion heated to $230^{\circ} \mathrm{F}$. for five minutes, and subsequently exposed in the incubator to a temperature of $122^{\circ} \mathrm{F}$., showed after twenty-four hours many Bacilli-tufts, which continued 
to grow for the next two days, the fluids themselves remaining clear. Then, in several of the tubes the fluids, to my surprise, grew rapidly turbid throughout. Others, which had not yet undergone this change, were saved therefrom by being removed from the incubator to a cool drawer; and some of them remained for a long time in my possession with the Bacilli-tufts floating in the clear fluid.

Two of this batch of tubes were examined early; and it was then found that the tufts were composed

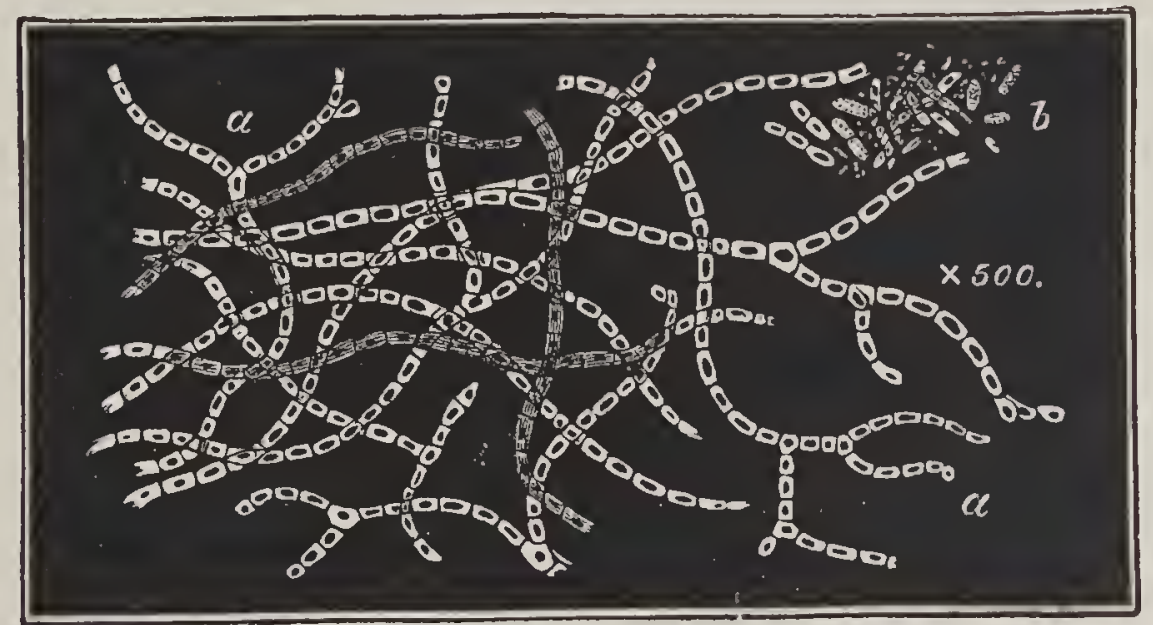

FIG. IO.

Other organisms from hay-infusions.

$a$, a. Mycelium of a Mould. $\quad$ b. Torula corpuscles.

of long Bacilli, and, further, that there were scattered among the threads a sparing number of mostly separate, small, ovoid Torula corpuscles (Fig. $\left.9,{ }^{2}\right)$. Here and there these were more numerous and aggregated into clusters. It was not till several months afterwards that I examined one of the tubes in which the contents had become turbid, and which had in the interval been also put aside in a drawer. I then found the fluid more than usually acid, swarming with short Bacilli; while, much to my 
surprise, the flakes were here composed of beautiful well-developed tufts of Mould (Fig. Io, $a, a$ ), which were growing over and more or less concealing the original Bacillus-tufts with Torula corpuscles (b). Another of these tubes was then examined, and its contents were found to be altogether similar. The vessels were made of combustion-tubing, and having been sealed when cold, the ends were very strong and were thoroughly sound. ${ }^{1}$

Superheated hay-infusion, when it ferments, invariably retains its characteristic odour, whether it has been heated in its natural acid state, ${ }^{2}$ or when neutralised, or made slightly alkaline by potash. In the process of fermentation an acid of some kind is generated, since even the slightly alkaline fluid may be found distinctly acid when we come to examine its contents microscopically. With regard to the organisms which are to be found in these fermenting hay-infusions, I have occasionally encountered, in addition to Bacilli of all lengths and Torula of various kinds, Diplococci, and Streptococci in the form of short chaplets (Fig. 9, ${ }^{4}$ ). In the fluids previously referred to in which the Torulae had developed into well-grown mycelia, I also met with a few organisms (Fig. 9, 3 ) which seemed exactly to correspond with Vibrio Rugula of Cohn. These I have never seen in such fluids on other occasions.

1 The supervention of a more vigorous fermentation, as shown by the turbidity, made the fluid more acid; and this change in the medium seems to have brought about the development of the previously existing Torula corpuscles.

2 The acidity is always low, mostly equivalent to $\mathrm{I}-2$ minims of liquor potassic per ounce. 
Milk.- If heated to $230^{\circ} \mathrm{F}$. ( $110^{\circ} \mathrm{C}$.) for threequarters of an hour or more, milk is found to be distinctly discoloured by the process. It is then of a light fawn-colour. A briefer exposure, however, to this temperature does not appreciably affect its colour.

After it has stood in the incubator for twenty-four hours or so, a cream-like layer is found at the surface, the upper stratum of which is yellow and dry, though it is dotted here and there with globules of fluid oil. At this stage the fluid below is still white and opaque; but where fermentation ensues, it gradually becomes more and more whey-like, and at last it may assume the appearance of mere dirty water. If left for a long time, the fluid may undergo other changes, and after a time become much discoloured. If the milk has been heated to $230^{\circ} \mathrm{F}$. for as long as 30 minutes or more, it may remain many days in the incubator at $122^{\circ} \mathrm{F}$. before it shows any sign of change.

When a specimen of superheated milk has fermented and become most notably altered in appearance, it still retains the simple odour of boiled milk. Its reaction, however, has changed, since it is always found to have become more or less acid.

When I first examined such a specimen of milk under the microscope, I was puzzled at not being able to discover any distinct or definite organisms amidst the milk-globules. It is true there was a teeming myriad of swarming particles everywhere, too minute to be individually recognised, and recognisable principally by their aggregate motions. 
These, however, might be organisms or might not; and, on the whole, I was inclined to take this latter view. The next specimen of fermented milk was, perhaps, examined with the aid of a betteradjusted light. At all events, I discovered therein a sparing number of Micrococci of the figure-of- 8 type; and these have since been recognised in every specimen of superheated milk in which I have sought for them-where the fluid has presented the other signs of fermentation. They are to be recognised best in portions of the whey in which the milk-globules are not so abundant. They have a provoking habit of placing themselves vertically beneath the cover-glass, when they look just like small milk-particles; but as they turn over their proper shape is seen, and we find them composed of a delicate protoplasm presenting a much lower refractive index than the milk particles with which they are intermixed. Once seen, therefore, they need never be confounded with couples of refractive milk-particles of about the same size, which are not unfrequently to be met with. The average length of these organisms is $\frac{1}{10,000} "$; but sometimes they are rather larger, and at others distinctly smaller (Fig. 9, ${ }^{4}$ ).

Where the fermentation is not vigorous, the organisms are very scarce and mostly small; where it is better marked, they are not only larger, but sufficiently numerous to be pretty easy of detection. On only a few occasions have I seen a chain of four elements; the organisms almost invariably exist in the binary form. The same organisms are amongst 
the first to show themselves when unboiled milk turns sour in open vessels, though under such conditions they are speedily succeeded by several other forms.

\section{Geneval Interpretation: present State of the Question in regard to Archebiosis.}

The germ theory of fermentation was adopted by M. Pasteur and the doctrine of "spontaneous generation" was rejected, in his celebrated memoir of $\mathrm{I} 862$, on the strength of three principal inductions from his experimental work, together with three corollaries severally deduced therefrom.

Arranged in order, they may again be stated as follows :-

Induction I. All guarded acid fluids remain barren after boiling. Corollary.-Bacteria, Vibriones, Torulæ, and their germs are killed when they are heated in acid fluids for two or three minutes to a temperature of $100^{\circ} \mathrm{C}$.

Induction II. Some neutral or faintly alkaline fluids, even though they are securely guarded, will ferment after boiling.

Corollary.-Certain Bacteria- or Vibrio-germs are not killed by being heated for two or three minutes in fluids to a temperature of $100^{\circ} \mathrm{C}$., when these fluids have a neutral or faintly alkaline reaction.

Induction III. All neutral or faintly alkaline guarded fluids remain barren after they have been heated for a few minutes to $110^{\circ} \mathrm{C}$. $\left(230^{\circ} \mathrm{F}\right.$.)

Corollary.-All Bacteria- and Vibrio-germs are killed, even in neutral or faintly alkaline fluids, when these are raised for a few minutes to a temperature of $110^{\circ} \mathrm{C}$.

These were the inductions and inferences to which 
the President of the British Association in 1870 gave his unqualified support, since it was in reliance upon Pasteur's views and researches principally that he proclaimed from his Presidential Chair the doctrine omne vivum ex vivo to be "victorious along the whole line."

If this could be said to have been an impartial verdict in 1870 , which it certainly was not, a totally different verdict will have to be given to-day. Since the same year (1870) I have on various occasions, and on various evidence, contended that the first, and third of these inductions were not good, and that the second corollary was neither warranted nor true. Additional and final proof of these positions has, I venture to think, been supplied in the foregoing chapters. I claim, therefore, to have shown that the grounds on which M. Pasteur, and the scientific world in general, following him, had accepted the "germ-theory of fermentation" and rejected the doctrine of "spontaneous generation" were altogether insufficient and in great part erroneous - as a wider experience with other materials, accurate experiments on the thermal death-point of Bacteria and their "spores," as well as new experimental conditions have shown.

The latest work on this subject emanating from the Pasteur Institute has left the evidence almost where it was before. Still a little change has been made, since Ch. Chamberland, a former assistant of Pasteur, and now sub-director of the Institute, in his Thesis for the degree of Doctor of Science published in 1879 , and also in a contribution to 
the Comptes Rendus for March 24 of that year, came to the conclusion that Bacillus spores could resist a rather higher temperature in neutral or slightly alkaline fluids than Pasteur had said. Yet he, after more than two years' work at this and related subjects, said in regard to the most resistant spores he had met with-that of the hay-Bacillus, and another, "Une température de I $5^{\circ}$ degrés les stérilise completement et très rapidement": meaning thereby, as his previous statements showed, that an exposure for a minute or so to that temperature invariably proved fatal to them.

Then, again, these statements by Pasteur and Chamberland are made concerning "spores" of Bacilli, and still more of such spores which have undergone some amount of desiccation. None of them, however, in the least explain the appearance of Micrococci, of Streptococci, or of Torulæ, whose frequent existence within the experimental fluids has been mentioned in the previous and in the present chapter, as occurring in fluids which had been boiled, and even heated to various points from $100^{\circ}$ to $125^{\circ} \mathrm{C}$. $\left(257^{\circ} \mathrm{F}\right.$.). There is, indeed, no existing evidence known to me to show that either of these three types of ferment organisms are able to resist, even for a few minutes, an exposure in fluids to $75^{\circ} \mathrm{C}$. $\left(167^{\circ} \mathrm{F}\right.$.). ${ }^{1}$

A brief reference must now be made to the experiments of Professor Tyndall, which did not profess to

1 One exception to this statement has since been made known, to which reference will be found on p. 229, Note I. 
do more than render support to the views of Pasteur (and not even to the whole of these), and which, in fact, by reason of his complicated methods and conflicting results, threw the whole subject temporarily into confusion. We shall then pass on to the consideration of new experiments of extreme simplicity, conducted with every possible precaution though under novel conditions, which have led to remarkable results of a kind calculated to meet any remaining objections, and make it plain that in these other experimental vessels there must also have been a de novo origin of the various kinds of living things found therein. 


\section{PART IV}

COMPLICATED METHODS AND CONFLICTING RESULTS

\section{CHAPTER XVIII}

PROFESSOR TYNDALL'S EXPERIMENTAL EVIDENCE WITH HEATED ORGANIC FLUIDS

I $\mathrm{N}$ i 875 Professor Tyndall began to work at this 1 subject, and announced his results early in the following year. ${ }^{1}$ He did not endeavour to ascertain the lowest temperature which would prove destructive to Bacteria, Torulæ, and their germs, though he came to the conclusion that they were always killed by being boiled for five minutes in organic fluids, and he seemed to imply that this result was irrespective of the precise degree of acidity or neutrality of the fluids employed. ${ }^{2}$ Since this conclusion as to the death of ferment organisms and their germs in infusions raised for a few minutes to $2 \mathrm{I}^{\circ} \mathrm{F}$. was based upon about 500 experiments with fluids of the most varied nature, Professor Tyndall seemed to feel considerable con-

1 Philosophical Transactions, 1876, pt. i. p 27.

2 Loc. cit. p. $5 \mathrm{I}$. 
fidence in its truth. ${ }^{1}$ So far as it went, therefore, his evidence on this part of the subject was entirely confirmatory of mine. Indeed, in the beginning of I 876, Professor Tyndall's views on this important subject were as much opposed to those of $M$. Pasteur as mine were ; we both disbelieved on good evidence, as we thought, in the survival of germs in boiling neutral or faintly alkaline fluids.

At this time M. Pasteur's positive results with some of such fluids would seem to have been forgotten by Professor Tyndall. At all events, not being able himself to get evidence that any boiled and guarded fluids would ferment, he attempted to throw discredit upon me because I had obtained such results. Forgetful of Pasteur's experiments above referred to, and apparently unaware of the confirmation which my experimental facts had obtained at the hands of many independent workers, he triumphantly brought forward a "cloud of witnesses" to convince the Royal Society and the world of science generally, as well as others, that my particular results in which fermentation had been made to show itself in boiled and guarded fluids were due to experimental errors into which it was conjectured that I had easily fallen, since it required all Professor Tyndall's great skill and long experience to avoid them. He strenuously denied that a

1 He says (loc. cit. p. 42) in experiments made with " urine, mutton beef, pork, hay, turnip, tea, coffee, hops, haddock, sole, salmon, codfish, turbot, mullet, herring, eel, oyster, whiting, liver, kidney, hare, rabbit, fowl, pheasant, grouse," amounting in all to several hundreds, five minutes' boiling was always found sufficient to produce complete sterilisation. 
certain experimental result could be obtained when strict methods were followed. It was as regards the question of fact, rather than in regard to its interpretation, that Professor Tyndall then did his best to throw discredit upon my work.

All this confident assertion and conjecture on the part of the new worker was based upon his belief, and is to be taken as the measure of his certainty at that time, that Bacteria and similar organisms, with their germs, were killed by being heated in fluids to $2 \mathrm{I} 2^{\circ} \mathrm{F}$., for a minute or two. It is, in truth, even now almost impossible otherwise to account for the continued barrenness of his 500 various fluids, placed, as he says, under conditions favourable for the multiplication of any organisms or germs which they might contain, not for days only, but for weeks and even months.

Professor Tyndall seems entirely to have misconceived the real aspect of the question as it stood before the scientific world in the beginning of 1876 . $\mathrm{He}$ unhesitatingly coincided with me as regards the only point which was really in dispute, viz. whether the supposed "omnipresent" ferment-organisms and their germs were killed by a brief boiling or not; while the fact which he called in question was the very point that had been abundantly confirmed, and was then generally admitted-whatever interpretation might have been put upon it by different experimenters. ${ }^{1}$ Indeed, what Professor Tyndall had been unable to achieve in the way of inducing

${ }^{1}$ For a list of such experimenters see Nature, February 10, 1876, p. 284 . 
fermentation in boiled and guarded fluids, had three years previously been brought about by me in the presence of a highly skilled and then sceptical witness, Professor Burdon Sanderson. He subsequently published his declaration ${ }^{1}$ that positive results, both with acid and with neutral boiled infusions, had been obtained without experimental flaw; yet in spite of this testimony, and without even mentioning it, Professor Tyndall sought to decry my experiments and set aside my results.

Meanwhile, almost at the time that the learned physicist was acting in this bewildering manner, one of the principal authorities on such subjects in Europe, Professor Ferdinand Cohn, was again confirming my impugned experiments at Breslau; and was obtaining, both with acid and with neutral boiled infusions, those evidences of fermentation which hitherto Professor Tyndall had strangely enough failed to reproduce. ${ }^{2}$ The fact was again fully admitted by Professor Cohn, though my interpretation of it was still questioned. It is therefore quite needless for me here even to cite the other investigators who had previously obtained similar results. This side of the question has, in fact, been so thoroughly settled by my experiments, and the numerous confirmations which they had received at the hands of others, that it would be

1 Nature, January 8, I873, and reproduced in Chapter xii.

2 "Beiträge zur Biologie der Pflanzen," I876, p. 259. This confirmation, after Professor Tyndall's denial, was very similar in its opportuneness to that of Professor Sanderson after Prof. Ray Lankester's earlier but similar denial and failure (Quurt. Journ. of Microsc. Science, January I\$33, vol. xiii. p. 74). 
waste of space for me now to dwell further upon this part of the subject.

It must be obvious that what was needed was all the definite evidence that could be obtained as to the thermal death-point, and as to the powers of resistance under different conditions, of fermentorganisms and their germs. This it was that induced me, then and later, to undertake long investigations bearing directly or indirectly upon this section of the problem.

Twelve months later we find Professor Tyndall ${ }^{1}$ announcing that he was then able to obtain the previously denied results. The behaviour of his recent infusions had completely stultified his previous position. He was no longer at issue with me and others in regard to the fact. The difference between us was now one of interpretation only. In spite of his previously much-vaunted 500 negative results, and the good evidence which they supplied as to the death-point of Bacteria and their germs, Professor Tyndall now endeavoured, as best he could, to cover his previous unfortunate position. The result was a complete change of front.

During all his earlier experiments, though operating in the midst of London in an air which he had himself not lightly stigmatised, and in many trials with all sorts of fluids, he had not come across a single germ which could survive the influence of boiling water for a few minutes. Desiccation of germs, according to Professor Tyndall's experience at this

1 Brit. Med. Journ., January 27, I877, p. 95. 
time, would seem to have been a phenomenon of the rarest occurrence; germs capable of resisting a short boiling must have been almost, if not quite, unknown.

But no magician with his wand ever wrought a more complete change than did Professor Tyndall by introducing a bundle of "old hay" into his laboratory. Henceforth there was evidence of fermentation in boiled fluids without stint; desiccated germs were said to be everywhere-germs capable of resisting even two, three, four, and more hours of boiling, as he thought, everywhere surrounded him and got into his infusions.

These, at least, were the hypotheses by which Professor Tyndall endeavoured to reconcile his earlier with his later results. But two things strike one as very unsatisfactory in regard to these hypotheses and his method of supporting their cogency.

In the first place it may be observed that the fact of his having introduced a bundle of "old hay" into the laboratory of the Royal Institution cannot be regarded as a satisfactory explanation of the results of myself and others who had been able to obtain fermentation in boiled fluids long before, without the aid of any such magician's wand as this which Professor Tyndall had chanced to employ.

Secondly, there is the very dubious nature of the evidence by which he sought to support his interpretation, and the absence of anything in what he had published on the subject which gave a definite and independent foundation to this interpretation. Thus, to take one illustration, in the 
Proceedings of the Royal Society, ${ }^{1}$ there is printed a note "On Heat as a Germicide when Discontinuously Applied," in which Professor Tyndall said : "Following up the plain suggestions of the germtheory, I have been able, even in the midst of a virulently infective atmosphere, to sterilise all the infusions by a temperature lower than that of boiling water. . . B Before the latent period of any of the germs has been completed (say a few hours after the preparation of the infusion), I subject it for a brief interval to a temperature which may be under that of boiling water. Such softened and vivified germs as are on the point of passing into active life are thereby killed; others not yet softened remain intact. I repeat this process well within the interval necessary for the most advanced of those others to finish their period of latency. The number of undestroyed germs is further diminished by this second heating. After a number of repetitions, which varies with the character of the germs, the infusion, however obstinate, is completely sterilised."

Noting by the way that the "character of the germs" has no other reality than Professor Tyndall chooses to infer from the obstinacy of the infusion in resisting sterilisation, it is only necessary further to point out that the above procedure and its results allow absolutely no conclusion to be drawn in favour of the survival of germs, except by ignoring the only other legitimate interpretation. The frequent repetitions of destructive heating, at or close to the boilingpoint, might, after a time, repress all tendency to

1 No, I78, vol. xxv. p. 569. 
fermentative change in a fluid with the same facility that it might destroy germs supposed to be successively awakening to life and activity. ${ }^{1}$ Whenever an investigator has decided beforehand that one of these possibilities is not worth thinking of as an interpretation, the problem to his mind, becomes, of course, a simple one. And amidst all the conflicting nature of his evidence Professor Tyndall never wavered in the steadfastness of his adhesion to the germ-theory. All through, with him, the question was always whether the germs were or were not killed with a given amount of heating. The other side of the question in dispute, the possible "generating" power of the fluids employed, was wholly ignored. He declared, in fact, in The Times (June 18, I877), in reference to

1 A fairer way of testing Professor Tyndall's notion may be found in some such manner as I have myself adopted. Thus, a hay-infusion was made in the ordinary way, which after filtration was found to be clear, of a dark sherry colour, and to have a neutral reaction. Nine tubes from three to four inches long and one inch in diameter were employed, which were thoroughly flambés. Having been rather more than half filled with the hay-infusion, the fluid in each was boiled for one minute and sealed during ebullition. These experiments were purposely made in the month of January, when the weather was rather cold, ranging from $32^{\circ}-45^{\circ} \mathrm{F}$. The tubes were kept outside the window, in the shade, and their position was reversed twice daily during four days, so as to allow plenty of time for the softening of any germs or spores that might be present either in the fluid or on the walls of the vessel. The tubes were then put into a can of water and raised with the water to the boiling-point-the boiling being continued for four minutes. During this second process of boiling two tubes were cracked, but the remaining seven were placed in the incubator at I $15 \mathrm{~F}$., with the following results: In $24-36$ hours the fluid in all the tubes had become slightly turbid and lighter in colour, owing to the presence of swarms of Bacilli. I have obtained very similar results on other occasions. 
his experiments, "there is but one interpretation. An interpretation which violates all antecedent knowledge is undeserving of the name." This was said by him in one of his most caustic letters in condemnation of my views and experiments, and in reply to my hint that there was another interpretation of his experiments which he entirely ignored.

It can be easily understood that a man who adopted this attitude, and was so overbearingly confident in regard to the truth of his own doctrines, would not trouble himself overmuch to meet all his opponents' views. In his next letter to The Times (July 24, 1877), in further condemnation of me, and written in his most dogmatic style, he said that when the discrepancy occurred between his earlier and his later experiments the possible influence of "the age of the hay " suggested itself to him. He then made the following remarks: "The hint was sufficient to cause me to seek through the country for samples of old hay. Lord Claud Hamilton was good enough to send me some from Heathfield. From Colchester I obtained hay five years old, and from other places hay in different stages of desiccation." Then comes the following surprising and boastful statement, "I hunted this hay-contagion down till I could place my finger on it, and I showed by experiments, long continued and laborious, the ravages it produced among infusions of all kinds."

I say this statement is a surprising one, because it must be remembered that at the time the actual "spores" of the hay-bacillus had recently become 
known. I had been making experiments in regard to their death-point, though absolutely nothing had been said by Professor Tyndall on the subject, and there was no evidence to be found in his writings that he had even seen any of these bodies. I had called public attention to this fact, and his reply in the letter from which I have just quoted is this: "What he says in regard to unseen germs is also said without knowledge. The germs are seen collectively, though the microscope may fail to resolve them [they are most easily to be seen through the microscope, as PI. I., Fig. 2 shows]. A patch of bluebells on a hill-slope is not the less a patch of bluebells because from a distance you are only conscious of their colour, and fail to distinguish the individual flowers."

That was the kind of treatment of the subject which satisfied Professor Tyndall. On the other hand, the reader may now look again at pp. $78-\delta_{4}$ for a record of my experiments with myriads of the actual Bacillus spores after they had undergone desiccation for over five years, as well as at pp. 75-78, giving an account of direct experiments on the death-point of these bodies when they had undergone a shorter period of desiccation. The results thus arrived at go to show that these real and veritable "old hay germs" - known to be there in their thousands-and after the most prolonged desiccation, were only found on one single occasion to have appeared to survive a heating to $100^{\circ} \mathrm{C}$. for twenty minutes. I say "appeared," because, as I have said, it is just possible that this particular experiment may have been a 
failure, owing to some accidental contamination having occurred after the nourishing fluid had been heated. ${ }^{1}$

In order to show that the view I have taken as to the unsatisfactory methods and conflicting results obtained by Professor Tyndall is not due to personal bias on my part, I think it well to quote here what was said on the subject by a writer in The Contemporary Review for April i877, under the signature of "Inquirer." And in regard to the competence of the author I am now at liberty to say that the writer was the late G. W. Hemming, a former Senior Wrangler, an eminent K.C., and later, for some years, one of the Judicial Referees of the High Court. He was a man of great intellectual power and sagacity, who knew all details of the controversy which had been taking place during the previous six or seven years, almost, if not quite, as well as I did; and this is what he had to say concerning Professor Tyndall's experiments :-

"The only other experiments to which we need refer, on the point now under consideration, are the very elaborate series conducted by Professor Tyndall since the Bastian-Sanderson report. These, like Pasteur's, have not been precisely repeated, but

${ }_{1}$ Professor Tyndall made so many erroneous statements in an article generally condemnatory of my views which appeared in The Nineteenth Century for January 1878 , that I was obliged to reply in the February number of the same review. After that, and previous to the present work, I have published nothing on the subject of Archebiosis save one chapter in my book, "The Nature and Origin of Living Matter," I 905. 
Professor Tyndall's position is such as to render a careful discussion, even of his isolated work, extremely interesting. It is perhaps to be inferred from the emphasis with which Professor Tyndall restricts

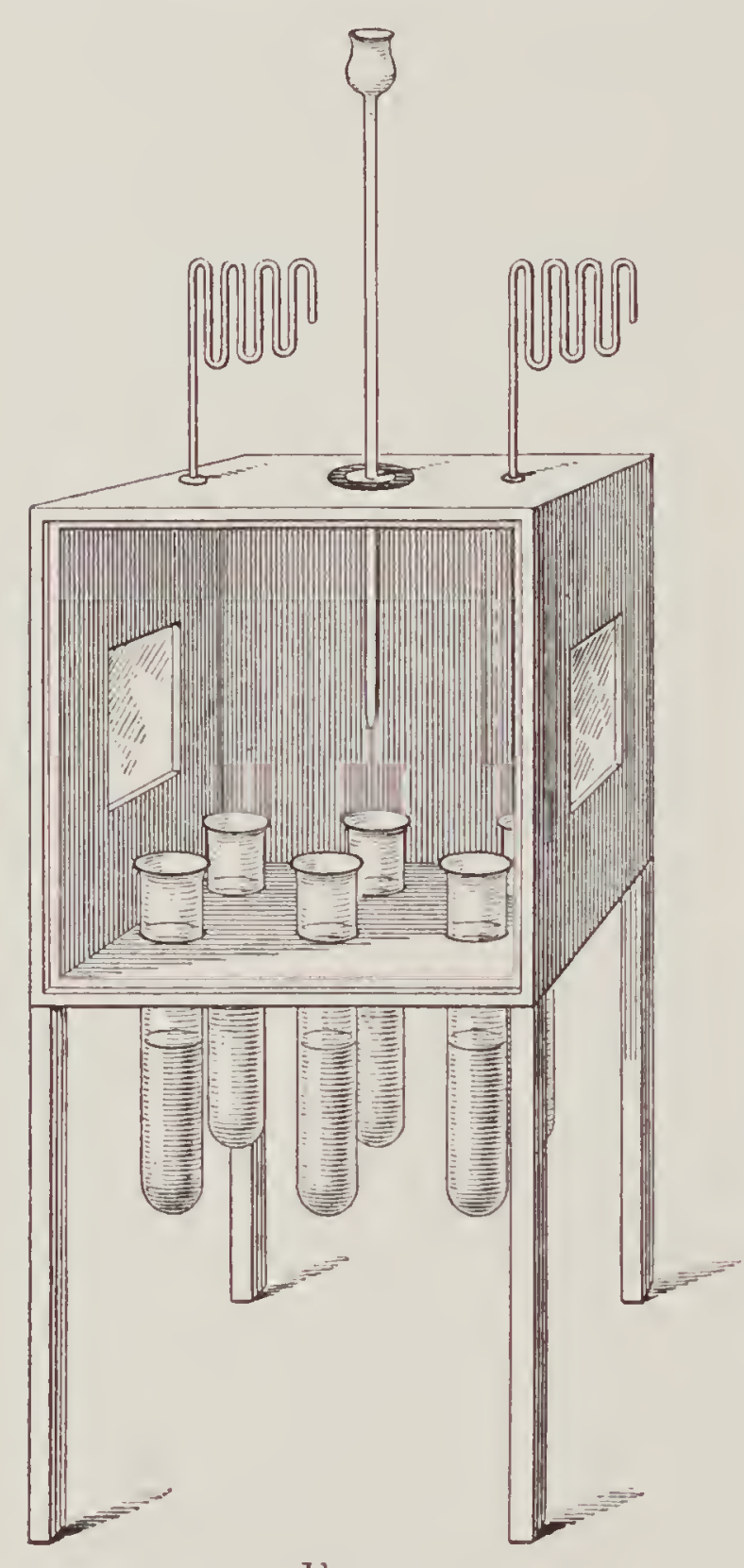

Fig. II.

Professor 'Tyndall's Experimental Chamber.

his statements to purely liquid infusions, that he has gone over and confirmed Bastian's turnip and cheese experiments, but he did at one time (whatever he may do now) maintain that no purely liquid infusion, whether acid or alkaline, could putrefy if boiled and protected from the contact of atmospheric dust. His experiments were conducted in two series, one described in a lecture at the Royal Institution early in 1876 , published in a more mature form in the Transactions of the Royal Society for that year; and the other in a lecture delivered at the Royal Institution on the igth of January of the present year, a report of which appeared in the British Medical Journal of January 27 th.

"The general method employed was to cement a 
large number of test-tubes through the bottom of a box with their lower ends protruding, so as to admit of the application of heat. The box was closed and made air-tight, and a pipette provided for filling the tubes with such organic fluids as might be desired. The air within the box was, as the professor considered, absolutely purified from germs by subsidence, the interior of the box being coated with glycerine, to imprison whatever dust might fall upon it; and the completion of this process was determined by the use of the electric beam. A connection with the outer air was established through bent tubes and plugs of cotton wool, which were thought to afford adequate protection against the intrusion of germinal matter.

"Why this roundabout and uncertain method of procedure was preferred to much simpler wellknown means it is difficult to say, but in the first series of experiments it led to no catastrophe. Various infusions were inserted in the test-tubes by means of the pipette, which was carefully guarded against the passage of air otherwise than through wool. The tubes were heated for a few minutes by immersion in an oil bath, and left in the laboratory to evolve life if they could. The temperature of incubation was not taken, but Professor Tyndall stated afterwards that a temperature of $90^{\circ} \mathrm{F}$. was 'generally attainable' in the laboratory, and that on mild days and in favourable positions the temperature to which the infusions were subjected reached over $100^{\circ} \mathrm{F}$. All of them remained barren, including any neutral solutions 
among them, which, according to Pasteur, ought to have putrefied.

"In the present year the same experiment was repeated, at the Royal Institution, with the opposite result. Most of the infusions became putrid, in some instances more rapidly than what were intended to be precisely similar infusions exposed to the outside air. The experiment was repeated with an improved arrangement of the pipette, which had fallen under suspicion from the conduct of the infusions, but still with the same result. Then the incoming air was cleansed not only by the woolfilter, but by calcining it by means of red-hot platinum, but still with no change in the result. Another box was then experimented on at Kew, and this time the infusions remained pure with one exception, after being kept for a time-not, we think, specified-in a temperature varying from $80^{\circ}$ to a little over $90^{\circ}$, a degree of incubating heat not the most favourable, but which has very often been found sufficient to develop life. The conflicting results of these experiments were ingeniously accounted for by Professor Tyndall-the failure at Kew being ascribed to a pin-hole, and those at the Royal Institution to the insufficiency of bent tubes, cotton-plugs, and red-hot platinum to keep out or kill living germs at Albemarle Street, though they are still relied on as sufficient in a purer atmosphere, and were found so last year in the Royal Institution itself. "The special theory resorted to to explain the contrast between the experiments at the Royal Institution in the two successive years is that in 1877 
that atmosphere was charged with bacterial germs in such excess as to be proof against subsidence, burning, and plugging, and that this excess was due to the fact that in one of the rooms of the Institution some old hay was to be found. How far this deserves consideration as a working hypothesis is a question which admits of difference of opinion; but Professor Tyndall would scarcely ask us to accept it without proof as the real explanation. Why bacterial germs in excess should make their way through a sieve ordinarily close enough to exclude them is not apparent. Neither is it quite obvious why the redhot platinum, which would burn up all the germs in ordinary air, should fail to do so when the contamination was excessive. And we are not quite sure that the subsidence of small particles from the. air is at all hindered by increasing their number; and if it were, it would not be a very serious matter to an observer furnished, as Professor Tyndall is, with an optical method of testing the purity of the air so delicate as to determine the instant when the last particle of germinal or other matter has deposited itself.

"Even when these difficulties are got over, there is no warrant that we know of for assuming that old dry hay will fill the air with swarms of bacterium germs. On the contrary, everything at present rather points to the hypothesis that desiccation would be fatal to bacteria and their germs, if they have any. ${ }^{1} \quad$ No doubt it is true, as the professor pointed

1 This is not quite correct. The writer probably had in mind the very definite statements made on this subject by Burdon-Sanderson 
out, that hard, dry seeds will stand an amount of heat which would utterly destroy them when they were once soaked through. Many persons would probably be able to confirm his experience, that a very dry pea may be boiled for hours before the moisture penetrates its hard coating; and it is common knowledge now that a considerable amount of dry heat will not destroy the power of germination in seeds which would yield at once to a moist temperature much below the boiling-point. Boiling a pea, therefore, seems a scarcely conclusive proof that bacterial germs, if there are any, can survive desiccation and induration, that theyare freely given off in this protected state from old hay, and that when they get into the air they have a special power, not possessed by their moister brethren, of resisting subsidence, penetrating cotton-wool, and enduring the contact of air calcined by red-hot platinum. All this may be worked out some day, but it will need something more than boiled peas or dried seeds to establish it.

"The true inference to be drawn from the two series of experiments seems to me to be that the discrepancy has arisen from some cause which neither the public nor the Professor himself have as yet any trustworthy means of so much as guessing at. The wise course would be to abandon for the future the unsatisfactory and complicated machinery employed in Professor Tyndall's mode of experimentation, and to resort to simpler means of exclud-

in $187 \mathrm{I}$ (Thirteenth Report of the Medical Officer of the Privy Council, p. 6I); his view, however, subsequently required much modification, as I have indicated on p. 75 . 
ing atmospheric dust; and as to the conflicting experiments of 1876 to 1877 , to let them drop quietly out of the argument until explained, the more especially as in the case of neutral or slightly alkaline fluids, many of them contradict the orthodox germ theorist, Pasteur, quite as emphatically as they do such heretics as Bastian and Huizinga, to say nothing of the mysterious Dr Sanderson, whom we dare not class either among the orthodox or the unbelievers, but who, whatever his theories may be, has borne manly testimony to the facts which he has observed.

"A very much more important statement is con- tained in the paper in the "Philosophical Transactions" of 1876 , into which Professor Tyndall expanded his lecture of that year. There it is distinctly, though briefly, alleged that Professor Tyndall has repeated the Bastian-Sanderson process, and that with purely liquid infusions he found in multiplied experiments that they remained uniformly barren. His own explanation last year was that Dr Bastian had allowed the gravest errors to invade his experimental work; that the life to which Dr Sanderson testified, in the case of the purely liquid infusions, arose from errors of manipulation; and this year he adds that even the celebrated Professor Cohn appears to have no adequate notion of the care necessary to be taken in experiments of this kind. To be consistent, he ought to have attributed the same carelessness to Pasteur when he obtained life in boiled neutralised fluids, a feat which Professor Tyndall declares impossible when due precautions 
are used and perhaps in some degree even to himself for overlooking the possible malignant influence of old hay.

"The worst of this kind of reasoning is not merely that it may be resorted to on one side as well as on the other, but that it tends to restore the habit of thought which once kept science dead for many centuries - the habit of appealing to authority in place of facts. Professor Tyndall may, or may not, ultimately prove to be on the right side, but we crave some other reason for it than the mere assertion that his undoubtedly high authority as an experimentalist (backed by Pasteur as to part of his experiments and contradicted by him as to the rest) entitles him to treat with contempt the counterauthority of Bastian, Sanderson, Huizinga, and Cohn." 1

1 In further illustration of some of the difficulties with which I had to contend, it seems worth mentioning that at the time while these discussions were going on Professor Huxley was one of the Secretaries of the Royal Society, Professor Tyndall was his intimate friend, and both were strong supporters of M. Pasteur, the truth of whose doctrines I had dared to question. My memoir entitled "Researches illustrative of the Physico-Chemical Theory of Fermentation, and of the Conditions favouring Archebiosis in previously Boiled Fluids," was read in June 1876 , and an Abstract of it was published in the "Proceedings" of the Society for that month, while the memoir itself was not printed, and was consigned to the "Archives" of the Society. In the following December communications from Sir William Roberts and Professor Tyndall, in supposed refutation of my experiments, were published with the least possible delay; as also was another communication from Professor Tyndall in February of the following year. His two long memoirs also were published in extenso, and as speedily as possible, in the "Philosophical Transactions" for 1876 and 1877. 


\section{PART V}

NEW EXPERIMENTS WITH SUPERHEATED

SALINE SOLUTIONS

\section{CHAPTER XIX}

OBJECTS AND METHODS IN THE NEW EXPERIMENTS:

INITIAL TRIALS

SO far as we have seen all micro-organisms, with $\infty$ the exception of Bacillus spores and certain Conferva living in hot springs, are admitted to be killed in the moist state when heated to temperatures ranging from $60^{\circ}-75^{\circ} \mathrm{C}$. $\left(140^{\circ}-167^{\circ} \mathrm{F}.\right) .^{1}$ Yet, as I have shown, not only Bacilli, but Micrococci, Streptococci, Torula, and other micro-organisms, have been found to be living, and, in many cases, actively multiplying, in organic fluids taken from closed tubes which have been heated to temperatures ranging from $100^{\circ}-125^{\circ} \mathrm{C}$. $\left(212^{\circ}-257^{\circ} \mathrm{F}\right.$.).

Further evidence in favour of the de novo origin of living things, in the face of these facts, would seem to be unnecessary. But the majority of persons

${ }^{1}$ One exception to this only is known to me. A Saccharomyces has been found which causes fermentation at i $83^{\circ} \mathrm{F}$., some account of which is given in the Journal of the Federated Institute of Brewing, vol. xi., No. 6, I905. 
still remain incredulous, and would apparently rather believe in the existence of some possible experimental errors than admit as true what they have long been taught to believe as impossible.

In the endeavour to throw further light upon this question of the origin of life I began again, after a prolonged interval, early in the year 1906, to make new flask experiments. It seemed desirable to make them approximate more closely to the condition of things that must have existed during the dawn of life upon our planet, by eliminating, as far as possible, all organic matter from the fluids with which experiments were to be instituted. The use of saline solutions of different kinds, made with freshly distilled water and with pure chemicals, naturally suggested itself, therefore, as a means of complying with this desideratum.

To get rid, as far as possible, ${ }^{1}$ of matter which had been fashioned in pre-existing living things would undoubtedly be a great advantage, and likely, in the event of positive results occurring, to prove additionally convincing to all unprejudiced persons. The chemicals employed would certainly be comparatively, if not absolutely, free from germs of Bacteria. They would certainly be as unlikely to harbour spore-bearing Bacilli as the freshly distilled water itself.

I say "freshly distilled" water, because it is well

${ }^{1}$ Chemists know how difficult it is to obtain solutions, however carefully prepared, which shall be absolutely free from minute shreds of cotton wool or other fibres. 
known that distilled water after it has stood for some time in ordinary vessels may be found to contain simple micro-organisms-growing more especially on the lower surface of the containing vessel. By using recently distilled water, however, we can almost get rid of all organisms-and certainly can get rid of spore-bearing Bacilli. So that the few micro-organisms that may chance to be present would not be of a kind in any way likely to complicate our results. If the thermophilic Bacteria are absent, as we are told, from ordinary tap-water, ${ }^{1}$ they are still less likely, as all must admit, to be found in freshly distilled water. And, even in regard to the relative purity of ordinary tap-water, the following facts should be borne in mind. MM. Pasteur and Joubert made an extensive series of investigations concerning the "germs of lower organisms" that were to be found in the waters of different regions, and among them in the water of the Seine (certainly very much fouler than any ordinary tap-water would be), and yet all they could say as to the capacity of resisting moist heat possessed by the organisms or germs found to be existing therein was this : ${ }^{2}$ -

"A drop of the water taken from above Paris, and still more when taken from below, is always fertile, and gives rise to the development of several kinds of Bacteria, among which there are some whose germs resist more than $100^{\circ} \mathrm{C}$., when in the moist state and in media which have not an acid reaction."

Subsequently to the date of these investigations,

1 See p. 69.

2 Compt. Rend., January 29, r 877, p. 208. 
Chamberland, the former assistant of Pasteur and the present sub-director of the Pasteur Institute, worked, as he tells us, for two years in Pasteur's laboratory in the preparation of his thesis for a Doctorate in Science, which was entitled, "Recherches sur l'origine et la développement des organismes microscopiques" - the object of these investigations being an attempt (deemed successful) to rebut the evidence adduced by myself and others in favour of the de novo origin of living organisms. During this work Chamberland thoroughly tested the ability of the spores of Bacilli to resist heat when immersed in water and in various acid, neutral, and alkaline infusions; and the conclusion to which he arrived touching these various fluids containing such germs was this :-

"A temperature of II 5 C. Sterilises them completely and most rapidly."

And the context shows that he means by this, as he subsequently repeated in the Comptes Rendus (1879, i. p. 659), that in a minute or two such a temperature suffices to kill all these spores when immersed in such fluids.

It behoves us to see again, therefore, what positive results can be obtained with fluids heated to I I $5^{\circ} \mathrm{C}$. and over, since this temperature has been so deliberately and authoritatively declared to be lethal for all germs or spores immersed in fluids.

Trials were at first made with several different solutions, nearly all of which contained an ammoniacal salt in combination with other bases or 
acids, and attention was finally concentrated upon a few solutions, the exact composition of which will be presently detailed. Some experiments have also been made with sea-water, as one of the most probable sources of the primordial living things which first appeared on this earth. Though not as free from germs as the saline solutions in distilled water, certainly sea-water is not a medium likely to contain desiccated Bacterial germs, and therefore the mere boiling of such a fluid ought to prove destructive of all living things contained therein.

The fluids with which experiments were made were contained in sterilised tubes, mostly of soft German glass, three inches long and one inch or rather less in diameter, and provided with a tapering extremity. The glass from which the tubes were to be blown was first well cleaned, and, naturally, during the process of making the tubes they became thoroughly sterilised. The advantage of this condition was maintained by the fact that the tubes were sealed as they were made, and that they, in all cases, remained closed until they were about to be charged with experimental fluids. When Tube after half-filled each tube was carefully sealed again in the flame of a Bunsen's burner, deposit of thus presenting the appearance shown in silica. Fig. I 2. The meaning of the deposit at the bottom will be subsequently explained.

The closed tubes were immersed in a can of water 
if they were merely to be heated to $100^{\circ} \mathrm{C}$., or in a solution of calcium-chloride for temperatures ranging from I I $5^{\circ}-130^{\circ} \mathrm{C}$. If temperatures above this were had recourse to, I have employed a colza oil bath, and then very great care is required to keep the bath at a definite temperature. Unless precautions are taken to slow the process of heating when the desired temperature is nearly attained, it will almost certainly run up four or five degrees higher than was intended. The calcium bath heats more slowly, and, of course, can be prepared so as to boil at any given temperature up to $\mathrm{I} 3 \mathrm{O}^{\circ} \mathrm{C}$.; so that, for short periods, the heating of the tubes in a bath of this kind can be kept at any definite point that may be desired. I have employed a deep can, narrowing above, the thermometer being fixed so that its bulb may be situated in the middle strata of the fluid. Not more than five or six tubes ought to be heated at the same time, as if one should burst the commotion caused may lead to the bursting of others. The shape of the can is then a protection. With careful sealing, however, and ordinary glass no accident of this kind should occur, though with the thinner tubes of uviol glass, presently to be mentioned, it has happened on two or three occasions.

When the tubes have been exposed to the given temperature for the time desired they should be quickly taken out of the bath with a large wooden forceps and allowed to cool. Then, after being cleaned and labelled, they are either placed in an incubator, at a comparatively high tempera- 
ture, or exposed to diffuse daylight at the ordinary temperature of the air.

\section{The Influence of Diffiuse Daylight.}

Comparative trials were made in order to ascertain whether the heat of an incubator at $90^{\circ}-100^{\circ} \mathrm{F}$. $\left(32^{\circ}-37^{\circ}\right.$ C. $)$, associated with darkness, or a much lower temperature, plus the influence of diffuse daylight, would prove most influential in leading to the appearance of organisms in the experimental fluids. I soon found that no general rule in regard to this could be laid down - that heat and darkness was more favourable with a few of the solutions, and that diffuse daylight, even with a very much lower temperature, was more productive with most of the others.

This favourable influence of diffuse daylight in promoting the growth, and possibly the origination, of micro-organisms is a new point, never, I believe, previously noted by bacteriologists. It seems, indeed, to be contrary to their generally accepted views, since we find $\operatorname{Dr}$ Allan Macfadyen saying in a lecture at the Royal Institution, delivered in June I900, that, "Direct sunlight was a most deadly bactericidal agent, and diffuse light was also injurious, though slower in action." 1

Its favourable influence was first discovered by me early in 1905, when studying the growth and multiplication of common Bacteria in a simple solution of ammonium tartrate in distilled water.

1 The Times, June I I, I900, and Proceedings, vol. xvi., p. $45 \mathrm{I}$. 
Sir William Ramsay, who kindly tested some of the solution employed, was unable to detect any phosphorus therein, and only "an excessively minute trace of sulphur, probably as sulphate." It seemed to me, therefore, that we had in this case to do with "the simplest kind of protoplasm," fashioned out of the mere carbon, hydrogen, oxygen and nitrogen of the ammoniacal solution, with the addition of an "excessively minute trace of sulphur."

The protoplasm was fashioned in this case under the influence of, and as a result of, the growth of the ordinary Bacilli, Cocci, Streptococci and Torulæ, with which the solution had been inoculated. This inoculation of the simple saline solution with common Bacteria and Torulæe further showed, as I then stated, ${ }^{1}$ that though such organisms were "capable of growing freely in the saline infusion without the aid of light," nevertheless, "light distinctly favours the process, since solutions, similarly inoculated and left exposed to ordinary daylight, have become turbid rather more quickly, even though the temperature to which the solution has been exposed has been about I I ${ }^{\circ} \mathrm{C}$. $\left(20^{\circ} \mathrm{F}\right.$.) lower than that of the incubator."

This kind of favouring influence was shown all the more plainly when other portions of the same saline solution were inoculated from the primary experimental fluid. The organisms in this secondary inoculation grew and multiplied less vigorously than their predecessors had done when in their own

1 See Knowledge and Scientific News, Aug. I905, p. 199. 
organic media. So that in the communication above referred to the following statement was made :-

"The growth of these less vigorous Bacteria is now decidedly less rapid, and seems only capable of occurring at all freely when aided by daylight. In the flask on the table the fluid will become slightly opalescent in four or five days, and this opalescence increases for a few days, when a sediment begins to form. But the fluid in the incubator may show no distinct opalescence even for a couple of weeks or more, though a very minute amount of sediment will accumulate."

In many of the experiments about to be recorded I have similarly found, as already intimated, some solutions very much more productive under the influence of diffuse daylight than when kept in the incubator at a temperature even $35^{\circ}-40^{\circ} \mathrm{F}$. higherso that this influence of diffuse daylight will be found to be a point of considerable importance. ${ }^{1}$

\section{Would it be advantageous in these Experiments to use Uviol or Rock-Crystal Tubes?}

A related point to that just dealt with has engaged my attention, though the observations hitherto made have not been sufficiently numerous or decisive to enable me to arrive at any definite conclusions. I

1 Yet the brief communication announcing this new contribution to "natural knowledge" was not, on the advice of referees, deemed worthy of a place in The Proceedings of the Royal Society in which I wished it to appear. It seems somewhat absurd that one of the senior Fellows of the Royal Society should have his communications referred to other Fellows who have made no special observations on the subject dealt with, and that they should practically decide, without communicating with the author, whether a paper is or is not to appear in The Proceedings of the Society. 
allude to the question of the desirability or the reverse of employing, in these experiments, kinds of glass which, like rock-crystal, are much more pervious to actinic rays of light than ordinary soft or hard German glass. I have made a few observations with sealed tubes composed of uviol glass, which is said to possess such properties, but they have not been numerous enough to enable me to say that their use has favoured the appearance of organisms or the reverse. The specimens of such glass at present obtainable ${ }^{1}$ have proved very variable in texture, and this led to several explosions occurring when the sealed tubes had been heated to temperatures over $130^{\circ} \mathrm{C}$. Further experiments with glass vessels of this kind should, however, be made; though perhaps the advantage to be derived therefrom will not be so great as might at first be imagined, if what M. Wilderman says is substantiated by further observations. He states that he is prepared with "experimental proof that the rays of light of all wave lengths act both 'chemically' and as 'heat-rays,' only in different degrees." 2

Of late, however, all the experimental vessels that I have been exposing to diffuse daylight have been laid on a table in front of an open window (day and night) having a north-east aspect; the table being so disposed as not to be touched by the rays of the early morning sun. In this way the window glass at least does not come as an additional barrier to the passage of actinic rays, while the commonly reputed

1 Through Messrs Isenthal \& Co., of $S_{5}$ Mortimer Street, W.

"Procedings of Royal Society, A, March I906, p. 274. 
injurious influence of direct sunlight on Bacteria is avoided.

Can Silicon replace Carbon, either wholly or partially, in Protoplasm?

What I have to say on this important subject may be prefaced by some quotations from my book "The Beginnings of Life," ${ }^{1}$ in which a brief reference was made to this question.

"Another subject now claims our consideration. Living matter being the result of a chemical combination of a certain kind, there is no absolute improbability in the supposition that the carbon usually existing in the living compound might be replaced by some other element. With the hope of throwing some little light upon this very difficult subject, I made several tentative experiments with saline solutions containing-in addition to nitrogen, oxygen, and hydrogen-some other element in the place of carbon. The element with which the carbon was replaced was either silicon, boron, chromium, aluminium, or iron. ${ }^{2}$ Except in those in which carbon was replaced by silicon, no living things have been met with in any of these solutions (after they had been boiled and the necks of the flasks had been sealed during ebullition). This result-taking it merely for what it is worth-is

1 Vol. ii., Appendix A, p. ix, I872.

${ }^{2}$ As I have already stated, these experiments were merely tentative. It is not supposed that solutions were employed free from all trace of carbon, existing as an impurity. 
extremely interesting and suggestive, since silicon is certainly the element which most closely resembles carbon, and which might therefore best replace it in compounds otherwise similar to those which constitute the basis of living matter. A silicon alcohol and ether has in fact been produced by Professor Wöhler, ${ }^{1}$ in which the carbon of the ordinary compounds is replaced by silicon. It is therefore deemed quite possible that silicon may take the place of carbon in certain forms of living matter. No absolute proof of this, however, can at present be advanced. What follows must be taken merely as an indication of the possibility of such an occurrence."

Reference was then made to an observation of my own, in which a mass of mould was found growing luxuriantly on the surface of some silicate of soda solution, contained in a corked bottle which had been unopened for about six months. Attention was also called to the previous observations of Messrs Roberts (afterwards Sir Wm. Chandler Roberts) and Slack on solutions of hydrate of silica, as recorded in the Quarterly Journal of Microscopical Science for I868 (pp. Io5-IOS), in which they say: "All the specimens of silica solution supplied by Mr Barff to Mr Slack, whether kept in bottles nearly full and corked, in bottles containing much air, or in open vessels, exhibited the mildew threads in the course of a week or ten days." After recording some further observations and experiments, they say, in conclusion, "The preceding experiments show the facility with which moulds will grow in a solution of

\footnotetext{
1 This is not quite correct. But see p. 279, Note I,
} 
pure silica in distilled water, and the way in which they may be artificially fossilised."

I then added the following details concerning a few experiments of my own with solutions containing silicate of soda :-

"In an experiment in which about ten minims of the weak solution of iron pernitrate and seven of sodic silicate solution were added to an ounce of distilled water, the fluid was boiled for fifteen minutes, and the neck of the flask was then hermetically sealed during ebullition. Some semi-gelatinous, reddish-yellow flakes were deposited during the ebullition. The vacuum being still well preserved, the flask was opened on the $35^{\text {th }}$ day, when the reaction of the fluid was also found to be still slightly acid. On one of the above-mentioned flakes there was found a minute whitish mass about the size of a small pin's head, which, on examination, was seen to consist of a mycelial tuft, having small but perfect filaments, though without any trace of fructification. The filaments themselves were about $\frac{1}{15000^{\prime \prime}}$ in diameter, but varied slightly in size, and contained a minutely granular proto. plasm without dissepiments. The numerous branches came off at right angles, and the whole organism had all the appearance of being a living fungus."

"The other silicate solutions in which organisms have been encountered were quite different in composition. They have been prepared by adding to one ounce of distilled water three grains of ammonic phosphate and about eight minims of sodic silicate solution. Such a mixture always had a slightly alkaline reaction, and it was sometimes used in this condition and sometimes after it had been rendered neutral or very slightly acid by the addition of a few drops of dilute phosphoric acid. The addition of the phosphoric acid seemed, however, to modify the result very much, since four slightly alkaline solutions with which experiments have been made have proved entirely barren, whilst three out of five solutions whose alkalinity had been neutralised by the acid, either contained organisms or spiral fibre masses. In all cases the solutions were boiled ${ }^{1}$ for from three to five minutes,

1 The silicates are held in solution very feebly, and, unfortunately, Q 
and the necks of the flasks were hermetically sealed during this process, and after the expulsion of all air. One flask, which had been prepared six months previously, and whose vacuum was ascertained to be scarcely if at all impaired, was found, when opened, to contain a fluid which still had a very slightly acid reaction. The numerous bluish-white flakes which it contained presented almost the same appearance as at first. Amongst these was found a minute whitish mass, about a line in diameter, made up of very delicate mycelial filaments, partly twisted around a cotton fibre. Near the centre of the mass was a large, brown, flagon-like body, about $\frac{1}{235}$ in diameter, from all parts of the surface of which issued the mycelial filaments whose ramifications went to constitute the rest of the mass. One or two smaller growths were also found attached to some of the flakes, as well as several distinct spore-like bodies of different sizes-mostly of a brownish colour, and having thick walls with granular contents. A group of fine spore-like bodies was also found; these being larger $\left(\frac{1}{66} "\right.$ " in diameter) and colourless, rather than brown. Their nature was altogether uncertain."

"A solution with an alkaline reaction which had been prepared at the same time, and opened after a similar interval, revealed no trace of spore-like bodies, or of organisms; and two other solutions containing sodic silicate and ammonic bichromate, whose periods of preparation and examination were also similar, were similarly unproductive. All four solutions had been prepared with distilled water taken from the same bottle."

\section{Preparation of the Solutions.}

All my recent work with these and other closely related solutions tends to confirm this fact of the great importance of the reaction of the fluids employed. Those which are very slightly acid have been found to be more productive than other

are in part precipitated, by the process of ebullition, in the form of bluish-white, cloud-like flakes, which show, on examination with high powers of the microscope, a very minutely granular composition. 
solutions similar, except that they contained a drop or two less of the dilute phosphoric acid, or what comes to much the same thing; a little more of the alkaline sodium silicate. The boiled acid solution will be productive, while the boiled slightly alkaline fluid is much more likely to be barren-again contradicting the data of Pasteur, founded on his more limited experience with this class of experiments. When I began in 1906 to repeat such experiments I did not at first attach sufficient importance to this point, and the result was that a considerable number of the tubes in which the fluids were slightly alkaline yielded negative results.

This point is, indeed, so important that great care is necessary in preparing the solutions. The ordinary sodium silicate solution is so tenacious that $\mathrm{Mr}$ Martindale ${ }^{1}$ has mixed the quantity sent to me with equal parts of distilled water. This at first forms a very opalescent fluid, but in the course of an hour or less the solution becomes a clear, waterylooking liquid. In the preparation of the experimental fluids, the same dropper has been used for the silicate of soda solution and for the other two fluid chemicals employed (namely, the dilute phosphoric acid or the liquor ferri pernitratis), when one or other of these has been required; though it has been cleaned with distilled water each time before being used for a different fluid. With the dropper employed, ten drops of water exactly equalled ten minims as measured; and the same was found to be

1 Of ro New Cavendish, W., from whom my chemicals and distilled water for these experiments have always been obtained. 
the case with ten drops of the dilute phosphoric acid and ten drops of the iron solution; but the drops of the sodium silicate mixture were smaller, so that ten of them only equalled seven minims of water. The different ingredients, whether solid or liquid, were added to freshly distilled water, and the experimental vessels were then at once charged, sealed, and heated to this or that temperature. When cool, the tubes were exposed either to diffuse daylight or to a temperature of about $95^{\circ} \mathrm{F}$. in an incubator, for varying periods; and always for weeks or months rather than for days.

\section{Examination of the Fluids.}

In all the cases in which sodium silicate enters into the composition of the experimental fluids there is a more or less abundant deposit, such as is shown in the experimental tube represented in Fig. I 2 ; but even after weeks or months have elapsed there is absolutely nothing to show to the naked eye, or to the naked eye aided by a good pocket lens, whether the tube does or does not contain living organisms.

In the case of an organic infusion, if organisms appear, the fluid in the course of a few days becomes cloudy or actually turbid, owing to their rapid growth and multiplication; or if that does not occur (as when the fluid has been much superheated, and what I have termed ${ }^{1}$ a "smouldering fermentation" is all that is set up) a small amount of sediment gradually collects where none previously existed.

1 Journal of the Limnern Society, No. 73 [Zool.], p. 3. 
But in these sodium silicate solutions the supernatant fluid invariably remains perfectly clear, month after month, though Bacilli, Micrococci, or Torulæ may be swarming in or upon the flakes at the bottom of the tube.

Another very important difference is this. In the organic infusions a large proportion of the organisms found will exhibit very active movements, but in the sodium silicate solutions the organisms are invariably motionless, scattered through or upon the flakes, and apparently originating where they are found. This holds good for Torula as well as for Micrococci and Bacilli, and must be regarded as a very important point when we see these motionless organisms occurring singly, as well as in small groups, throughout the flakes. When hundreds and often thousands of these motionless organisms are thus to be seen; and when it is borne in mind that no organisms whatever are to be found within other of these tubes (serving as "control" experiments) if their contents are examined within a day or two after they have been heated, what conclusions are we to draw? If organisms are not there at first, after the process of heating, and if, after an interval, they are there in abundance and are invariably stationary, clearly they must have developed in the sites where they are found.

Is there any chance of contamination having taken place after the heating? None. The procedure has been this. The end of the tube has been cut off, and if the orifice is narrow some of the deposit is shaken out on to a clean microscope slip, which 
has just been sterilised by passing it four or five times through the flame of a spirit lamp, and the deposit is at once covered by a cover glass, which has been similarly treated. If organisms are found, they are generally photographed at once, or else after they have been stained by drawing a drop of a solution of eosin or of gentian violet beneath the cover glass. In case the specimen is reserved for future more careful examination, or with a view to see whether the organisms found will develop further, the cover glass is at once surrounded with some paraffin melting at about $105^{\circ} \mathrm{F}$.- which will sometimes prevent evaporation for several weeks, and allow Bacteria or Torula to multiply, or the latter to develop hyphe, and thus remove any lingering doubt as to whether the organisms are or are not really living.

Where the aperture of the tube is larger, the only variation is that some of the deposit is withdrawn from the tube by a pipette, which has just been sterilised in the flame of the spirit lamp, and from it transferred to the sterilised microscope slip.

With the solutions which I shall name presently, organisms will generally be found in the first one or two specimens taken from the tube, because I shall refer only to the solutions which have been found to be most productive. As I have said, the organisms will all be motionless, but their numbers, looking to the character of the fluids employed, will clearly testify to the fact that they must have developed within the tube after it had been heated-moreover, in the specimens mounted as I have described, the 
organisms will often be found to multiply beneath the cover glass, and in the case of some Torulæ, instead of continuing to divide, they may begin to develop hyphæ, on their way to the formation of the characteristic mycelia of common Moulds.

Still, a word of warning is required concerning the examination of deposits taken from saline solutions generally. Bodies are frequently met with simulating Micrococci and Diplococci, and even Bacilli and Torulæ, which are yet nothing more than lifeless inorganic concretions-they are really incipient or abortive crystals. These bodies are especially common in the flakes of silica where the solutions have been heated to high temperatures, and have subsequently been kept for several weeks before their examination. In some cases they most closely simulated Micrococci or Bacteria, and they have been distributed through the flakes in just the same manner that real Micrococci or Bacteria are distributed in other cases.

These high temperatures cannot be borne even by saline solutions without some amount of change and degradation taking place, as evidence of which I may cite the following facts, though, of course, many other changes that occur would not be of a kind to reveal themselves by any visual characters.

In two tubes which contained a weak though perfectly clear solution of ammonium carbonate and sodic phosphate, after being exposed to $165^{\circ} \mathrm{C}$. for $2 \mathrm{O}^{\prime}$, the fluid was noted at the time to have become "rather turbid and to contain a distinct white 
curdy deposit"; while in two tubes which contained an originally clear solution of boric acid with ammonium phosphate, after a similar heating the fluid was found to have become "rather turbid, with a comparatively large amount of a fine whitish sediment." Again, specimens of sea-water heated to $160^{\circ} \mathrm{C}$. for twenty minutes, "became opalescent, and several small whitish shreds were to be seen in each of three tubes." In other cases no such change has occurred, though here, as in the instances above cited, the tubes were composed of the same kind of soft German glass. It is needful to mention this, because other experiments have shown that the use of different kinds of glass may also alter the results of mere heating. Thus, recently, three tubes containing fresh sea-water were heated at the same time to $\mathrm{I}_{20} \mathrm{C}$. for ten minutes. Two of the tubes were of common glass, and in them the sea-water after the heating was found to have remained quite clear and free from deposit; while in the third, which was of uviol glass, the fluid was full of very minute glittering iridescent scales. Again, a faintly acid sodium silicate solution, after having been heated to $100^{\circ} \mathrm{C}$. for ten minutes in a uviol tube, showed at first no apparent deposit of silica, and only a very little appeared later on; and, when the fluid was examined on the fourteenth day, it was found to be slightly alkaline, rather than appreciably acid, as it should have been in a tube of common glass. 


\section{The Solutions employed and the Results of their Examination}

I restrict myself here to the mention of those saline solutions whose employment in my experiments have yielded more or less uniformly positive results. They are variants of the solutions already referred to as having been experimented with in I $871-72$, in reference to the question whether silicon could or could not replace carbon in the constitution of protoplasm. For the present it need only be noted that in the solutions themselves carbon did not exist, except it might be in the form of some impurity contained therein-either in the distilled water, or in one or other of the chemicals employed. In each case, however, silicon was provided and present, in order that it might possibly take the place of carbon in any organisms that appeared in the solutions.

The first solution dealt with was one containing sodium silicate, ammonium phosphate, and dilute phosphoric acid. At first these ingredients were used in the proportion of six drops each of the first and third, and six grains of the second to the ounce of distilled water. Later, after some comparative trials with different strengths of such solutions, each of which was inoculated with Bacteria, simply to test the relative virtues of the solutions as mere "nourishing" fluids, I was induced to use four drops and four grains, in the place of six, to the ounce of water. In each case this gave 
a fluid which was faintly alkaline, and the results with them have not been so successful, at all events for the production of Bacteria, as when I diminished the amount of the silicate, and thus rendered the solution faintly acid. The first solution I recommend, therefore, is this :-

A. Sodium silicate, two, or three, drops. ${ }^{1}$

Ammonium phosphate, four, or six, grains.

Dilute phosphoric acid, four, or six, drops.

Distilled water, one fluid ounce.

Another is the solution containing a rather larger amount of sodium silicate, its composition being as follows :-

AA. Sodium silicate, four, or six, drops. ${ }^{2}$

Ammonium phosphate, four, or six, grains.

Dilute phosphoric acid, four, or six, drops.

Distilled water, one fluid ounce.

The next solution is one containing sodium silicate and pernitrate of iron, two varieties of which have been used, the first of them having a faintly acid and the second a slightly alkaline reaction. Their composition is as follows :-

B. Sodium silicate, three drops. ${ }^{2}$

Liquor ferri pernitratis, ${ }^{3}$ eight drops.

Distilled water, one fluid ounce.

BB. Sodium silicate, six drops. ${ }^{2}$

Liquor ferri pernitratis, eight drops.

Distilled water, one fluid ounce.

1 In using the very convenient mixture with equal parts of distilled water and sodium silicate, these numbers must, of course, for that ingredient, be doubled. See p. 243 also concerning the dropper.

2 Double the number for the dilute solution.

s Of the British Pharmacopœia. 
Of these two the latter has principally been employed, as I have only more recently made some trials with the former solution.

In speaking of the results obtained in the different experiments now about to be detailed, as well as in those to be recorded in the next Chapter, it will be convenient to refer to the solutions merely by the capital letters by which they are preceded.

I at first made some tentative experiments in which the vessels were not hermetically sealed. Small two-ounce flasks were employed, provided with new corks. The flasks were not flambés; they were simply cleaned with distilled water. A small wedge-like portion was cut out from the lower border of each cork, so that when the flask was charged with its experimental fluid and was being heated the cork might be, in a measure, purified by the steam issuing from the flask. This was brought about by placing the cork loosely in the neck of the flask, so as to allow the steam to find an exit between it and the nick in the cork. When the solution had boiled for the requisite time, and while still boiling, the neck of the flask was grasped with a large wooden forceps, while the cork was firmly pressed home. In vessels so prepared I have seen ebullition continue for six or seven minutes, when a cold object was applied to the upper part of the flask. Later on, air makes its way into the flask, either through the cork or in minutest bubbles between it and the neck of the flask, as may be seen on examination with a lens. This method 
was adopted rather than the use of flasks the necks of which were plugged with cotton-wool, because it permitted samples of the silica to be taken from time to time with a sterilised pipette, for examination, with rather less risk of contamination than would have resulted from the withdrawal and replacement of plugs of cotton-wool.

Reference will be made to a few of these experiments (which can be so easily repeated by others), because organisms undoubtedly occur in them rather more freely than they do in superheated and hermetically sealed vessels; and yet that this is not due to infection seems shown by the fact that just the same kind of organisms that are met with in the one case are also found in the other, as I shall subsequently show-the difference being that they are rather less abundant, and appear after a longer interval, where closed tubes and high temperature have been had recourse to.

An interesting point not yet mentioned is, that the organisms found in these particular solutions containing silica, when heated to high or comparatively low temperatures are, in part, organisms of a somewhat unusual character, of which I can find no description in bacteriological works.

Results obtained with Saline Solutions which had previously been heated in Corked Flasks to $100^{\circ}$ C. for ton minutes.

In Plate II., Fig. 5, A, a number of Bacteria, together with two or three Torulæ, are seen, which 



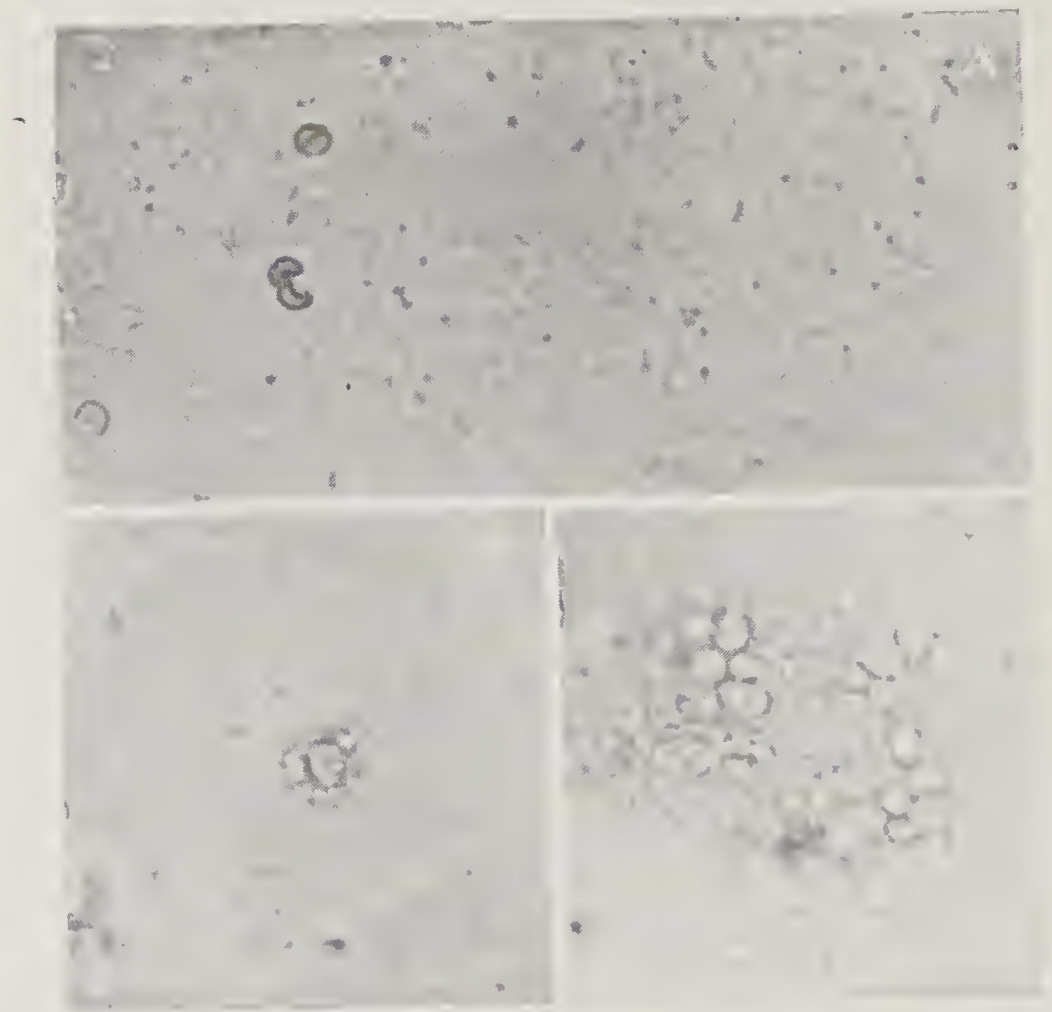

FI1.. $5(\times 700)$

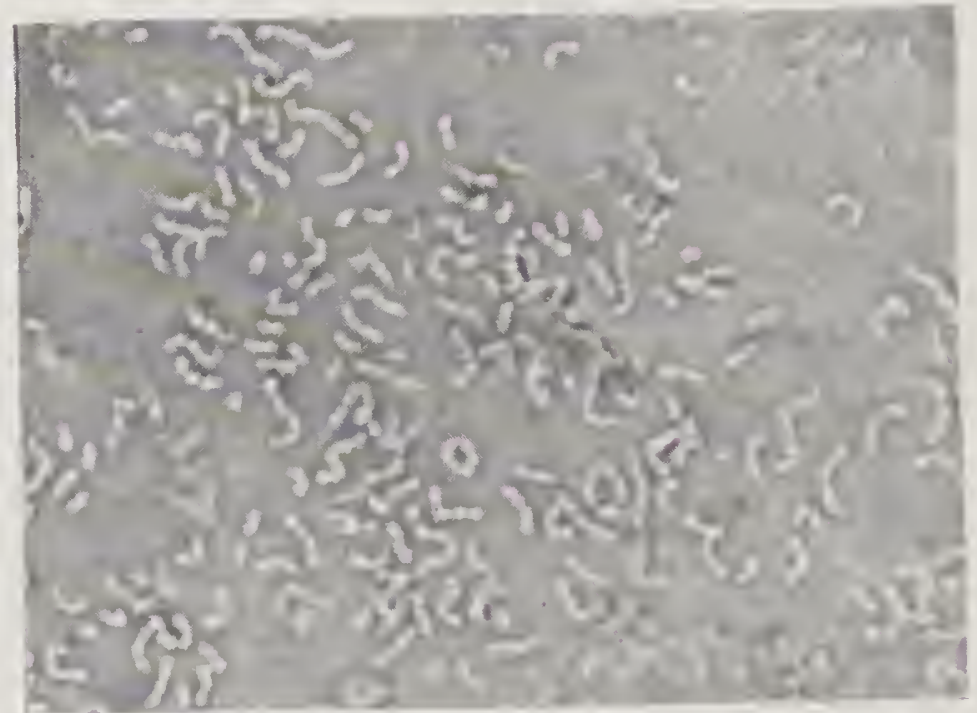

Fir. o ( $>500)$

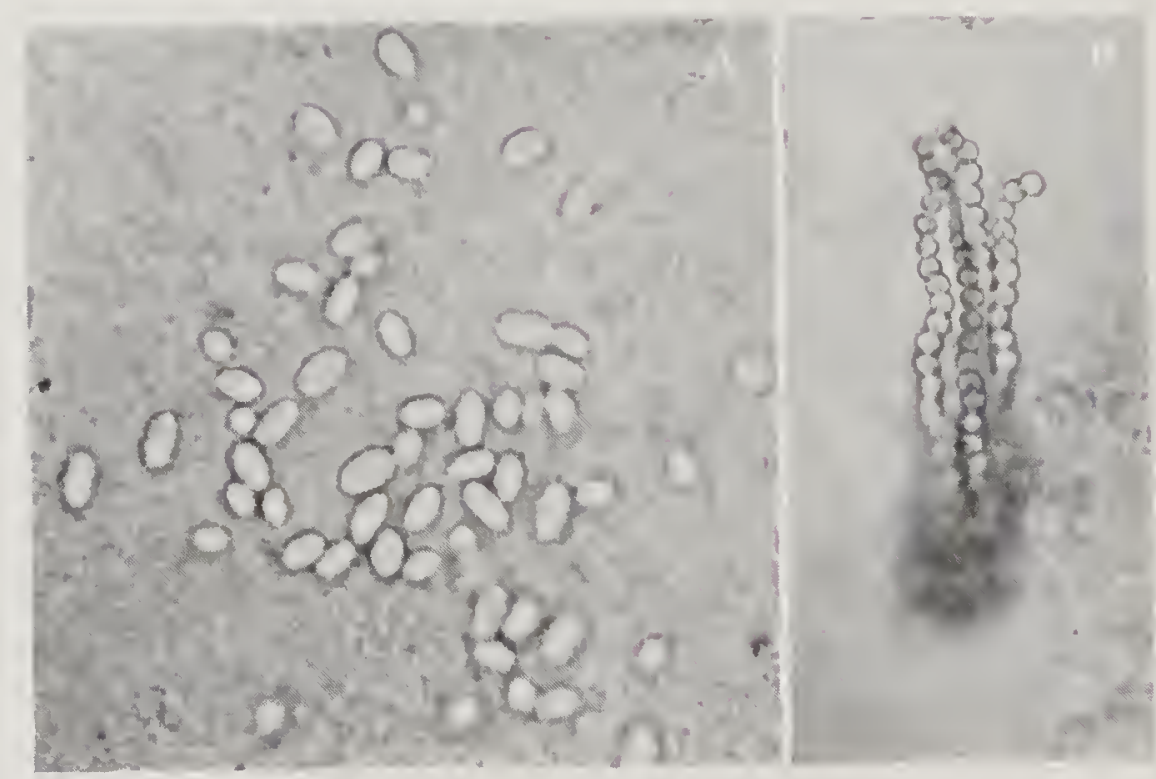

1.11. $7(\times 500)$

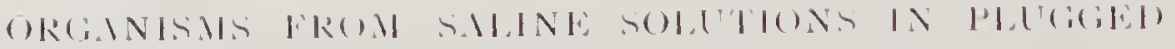

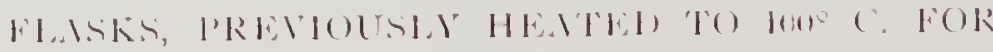


were found abundantly in and on flakes of silica, taken from a boiled A solution in a corked flask, that had been exposed to diffuse daylight, during bright and warm weather, for only two weeks.

While in Fig. 5, B, there is shown, from an AA solution which had been exposed to light for two months, during colder weather, what I have very frequently seen-that is, a Torulæe corpuscle surrounded by Bacteria. At other times, groups of such corpuscles, with surrounding Bacteria, are to be seen, as in Fig. 5, C, which was found in another flake of silica from the same solution.

In Fig. 6 a number of comma Bacilli or Vibriones are shown, such as were found on the surface of flakes of silica taken from an A solution which had been exposed to light for three weeks.

In a BB solution that had been exposed to light for only fourteen days, during hot weather in the month of June, a number of rather large colourless Torula corpuscles, some of which are shown in Fig. 7. A, were found, either singly or in groups, rather sparingly distributed through the flakes of silica. Associated with the Torulæ there were some small groups of Micrococci.

Other brownish Torulæe corpuscles are shown in Fig. 7, B, which were taken from a B solution that had been exposed to light for two weeks during warm weather. They had a tendency to grow in single rows, or in groups of rows, as shown in the figure.

Torulæ have generally been found distinctly more frequently in flasks that have been exposed to light than in those which have been placed in a dark 
incubator-where, however, they would also be exposed to a much higher temperature.

In the next plate organisms of a very unusual character are shown, which were found in a BB solution that had been in the incubator for about fourteen days. By that time it, and the deposit contained therein, had assumed a red-brown colour, instead of being, as usual, of a pale yellowish tint. From being slightly alkaline, too, the fluid had become distinctly acid. In the granular matter of the flakes there were a number of groups of free Micrococci, but mixed with them were others contained in distinct loculi. In their early stages these bodies are shown in Plate III., Fig. 9, A and B. In A a single loculus is shown, stained with eosin and containing several Cocci; while in $B$ three of the loculi, unstained, are to be seen. Ultimately they go on to the production of large sponge-like masses, one of which is represented in Fig. 8. In these large masses the walls of the loculi are often very indistinct, and they tend to disappear, as may also be seen from Fig. Io. In this latter specimen another very unusual character is found-that is, the development from the Cocci of long and generally much twisted threads. This development of threads sometimes occurs from the Cocci contained in single loculi, an example of which is shown in the stained specimen represented in Fig. 9, C, from which two threads are issuing.

In the next chapter I shall have to show loculated Micrococci of the same kind, which had been taken 


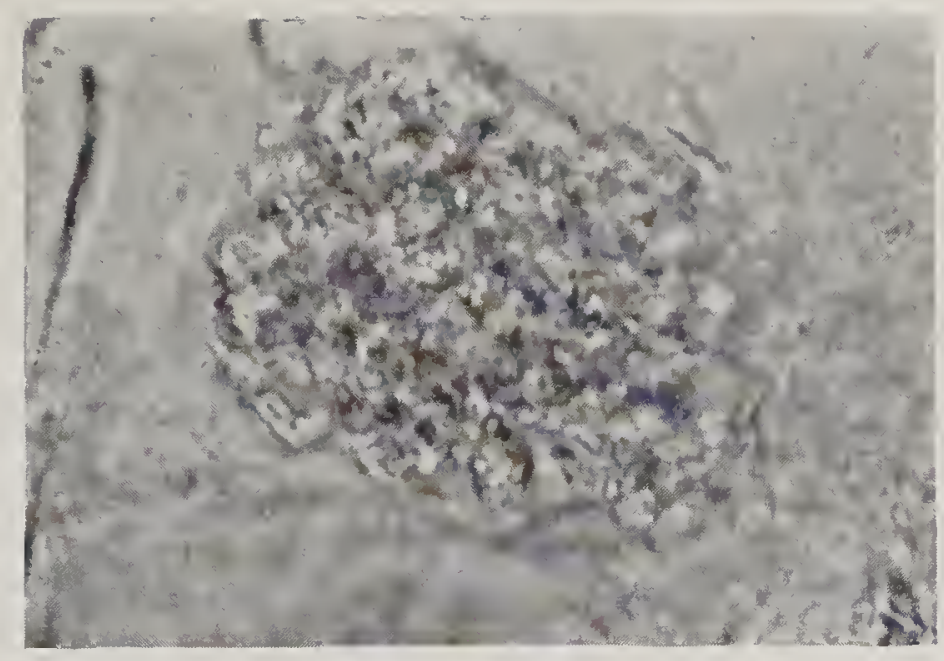

FIG. $8(\times 700)$

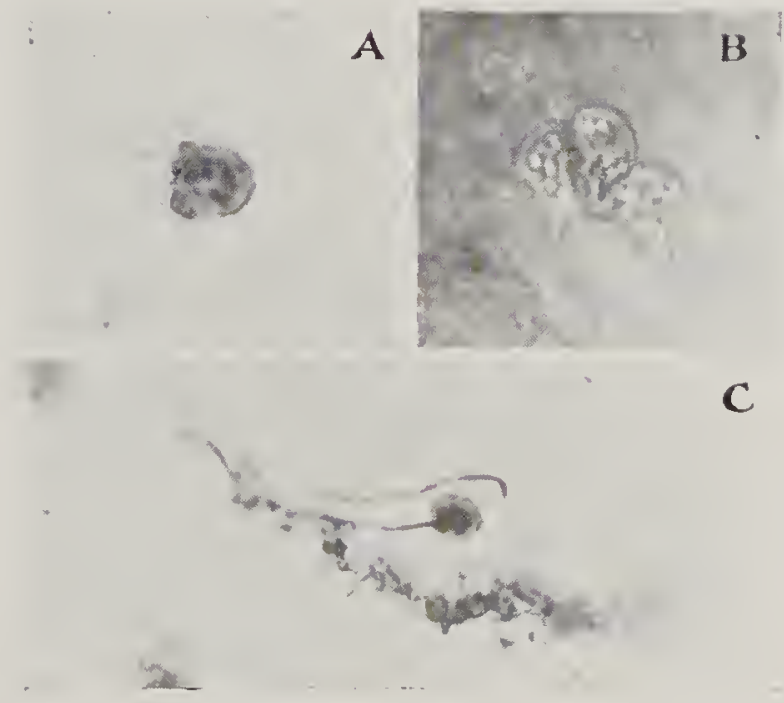

FIG. $9(\times 700$ AND 500$)$

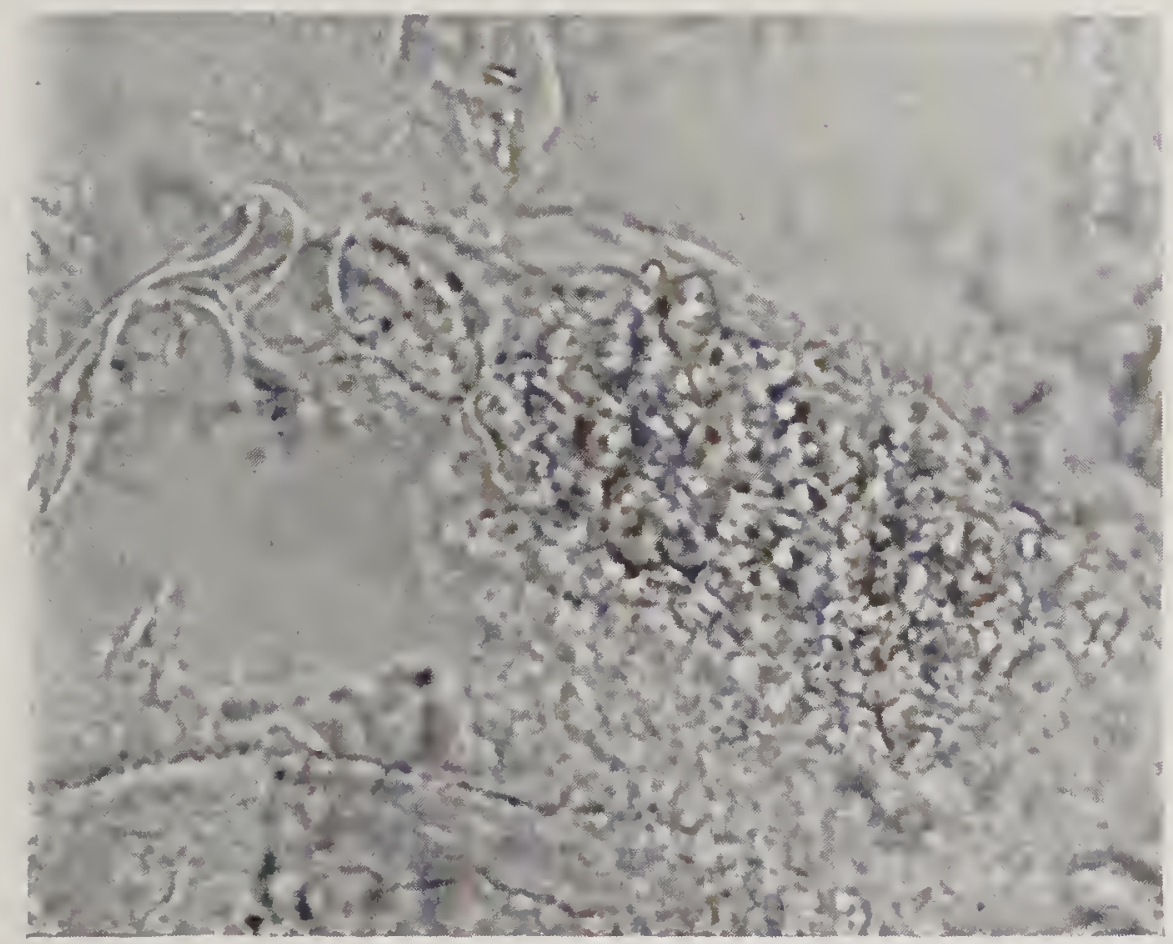

FIG. IO $(\times 700)$

OTHER ORGINISMS FRON SALINE SOLUTIONS IN

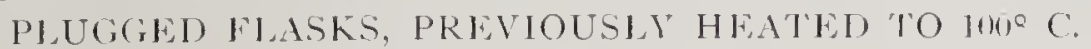
FOR TEN MINUTES 

from sealed tubes previously heated to $\mathrm{I} 3 \mathrm{O}^{\circ} \mathrm{C}$. for twenty minutes. No such bodies are to be found depicted in any of the bacteriological works to which I have referred. The loculi are, of course, something like what is to be found in Leuconostoc, which Lehmann and Neumann say (loc. cit., p. I23) " is only a Streptococcus, with, at times, enormously thick capsules." One of its forms, too, is spoken of (p. I $5 \mathrm{I}$ ) as assuming a "frog-spawn"-like mode of growth, which, on an extremely minute scale, is not unlike what is to be seen in Fig. 8. But then in our organism the contents of the loculi are Micrococci and not Streptococci. No such twisted threads are known to issue from Streptococci-nor, indeed, previously from any other Cocci, so far as I have been able to ascertain. The threads seem to be provided with no dissepiments; they are, therefore, not like the mycelia of a Mould, and seem to resemble much more closely the filaments of an Actinomyces. These are surely new organisms found in a new habitatand it may well be asked, whence have they come?

Results obtained with Saline Solutions which had previously been heated in hermetically Sealed Tubes to $100^{\circ}$ C. for ten minutes.

We now come to experiments of a stricter order, where previously sterilised tubes have been used, which, when charged with the experimental fluids, have been hermetically sealed, before boiling them in a can of water for ten minutes.

In one of these tubes, charged with some of the 
A solution, that had been exposed to light for five weeks, the flakes of silica were found to be crowded with Bacteria mixed with inorganic concretions, rather more refractive, but otherwise very difficult to be distinguished from the many solitary micro-organisms. When these latter, however, have multiplied much in any one place so as to form groups (which were numerous) such as are shown in Plate IV., Fig. II, their nature become perfectly plain. The Bacteria may be seen in such groups in different stages of development. Only one specimen was taken from this tube, and in that no other kind of organism was found.

In another tube charged with the AA solution which had been exposed to light for five weeks, during the same period, many Bacteria and a few Torulæ were found distributed through the flakes of silica; while on the surface of some of them there were many groups of more or less twisted organisms, such as are represented in Fig. I 2, which seem to be motionless Vibriones. These bodies were, in other places, mixed with organisms such as are shown in Fig. I3, that must be regarded either as branching Bacilli, such as are depicted by Lehmann and Neumann in Plate 61, Fig. viii., of their "Principles of Bacteriology," or else as incipient specimens of Actinomyces.

In another tube charged with the AA solution and exposed to light for five weeks, in addition to Bacteria there were found in the flakes a number of minute incipient Moulds, such as are shown in Plate V., Fig. I4. In A the little Mould seems 


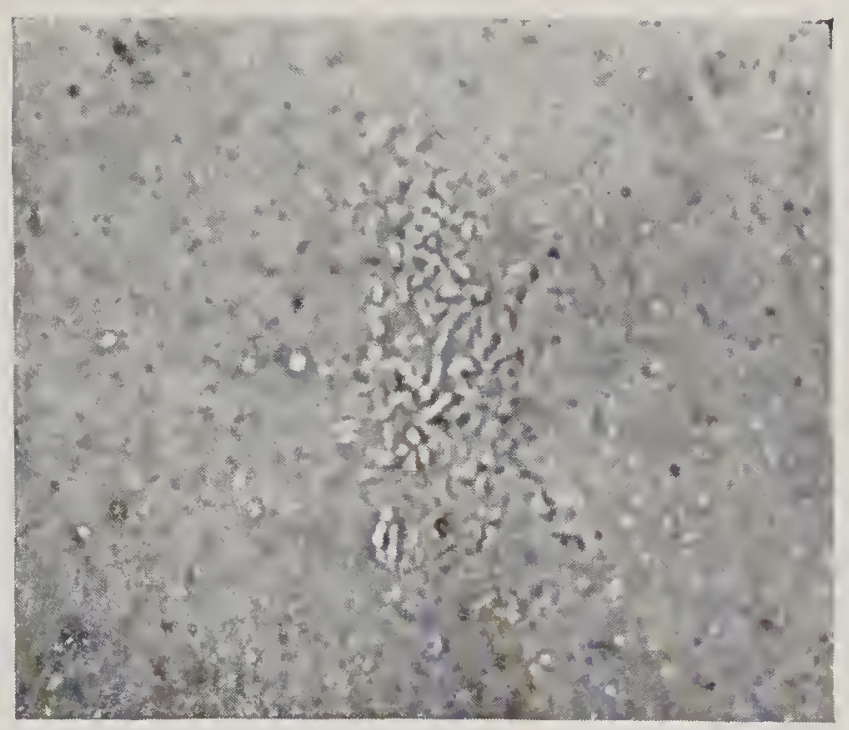

FIG. II $(\times 700)$

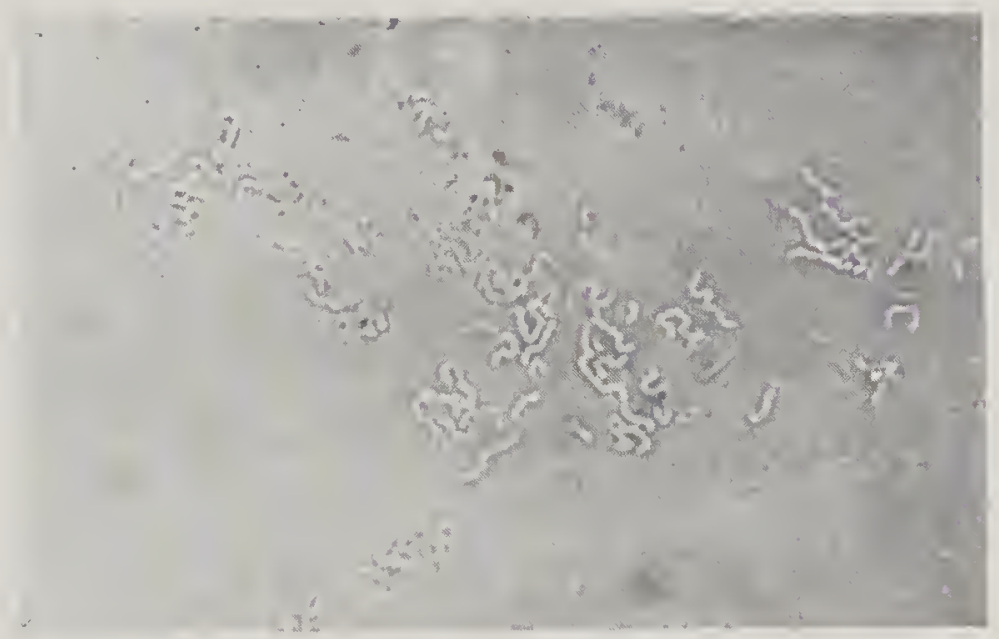

FIG. I2 $(\times 500)$

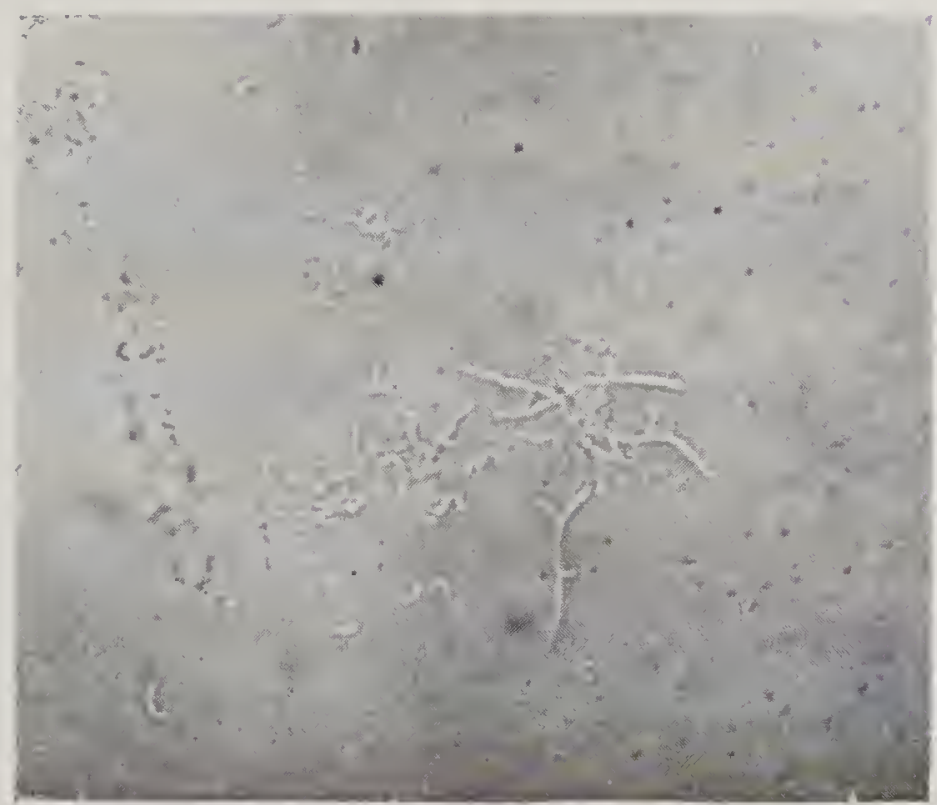

FIG. $I_{3}(\times 5 C)$

ORGANISMS TAKEN FROM PREVIOUSLY CIOSEL TUBES WHICH HAI) IEEN HEATED TO $100^{\circ} \mathrm{C}$. FOR TEN MINUTES 

connected with a body, at one extremity, of doubtful nature, though the other extremity is bifurcated -the longer of the two branches tapering away out of focus. In B the more usual form of these commencing Moulds is seen-in which both extremities taper away from a more expanded middle portion.

In another tube charged with some of the same AA solution the fluid was first boiled over the flame for about three minutes, so as to expel all air; the tube was then sealed during ebullition, and subsequently boiled for seven minutes in a can of water. This airless tube was then exposed to light for five weeks by the side of the other. On examination the flakes of silica were found to be crowded with cocci-like particles, though no distinct Bacilli were seen. It was impossible to decide from their appearance whether the separate cocci-like particles were organic or inorganic in nature. But I think it most probable that the majority of them were inorganic. There were, however, a few Torulæ to be seen here and there, together with a small number of incipient Moulds in different stages of growth. Three very early stages of these are shown in Fig. I 5, A, B, and C. In B there is a body like a corpuscle at one extremity, as in Fig. I4, A. It is probably an inorganic particle in both cases. In Fig. I 5, D, a comparatively long Mould is shown from another of the flakes, which was slightly twisted so that much of it is out of focus, though both its tapering extremities are distinctly shown. These bodies certainly 
originate from no Torulæ corpuscle. They seem to develop from some minute primordial particles.

After two samples of the flakes had been abstracted with a sterilised pipette, this tube was closed-now, of course, containing air-and was put aside in a box for three weeks. When examined again after this interval, some of the flakes of silica showed numerous groups of rather large and very typical Micrococci, such as are shown in Fig. 16, though the fluid above the deposit remained, as usual, perfectly clear.

Three other experiments previously made with the AA solution in airless retorts, which had been heated to $100^{\circ} \mathrm{C}$. for fifteen minutes and subsequently exposed to light for about two months, also showed either Bacteria alone or these mixed with a few Torulæ, in the flakes of silica. The experiments made in $187 \mathrm{I}$, in which organisms were found, as recorded on an earlier page of this Chapter (p. 24I), were also made with airless flasks; so that none of the organisms found in either of these vessels could have derived carbon from the carbonic acid existing in the air, seeing that the great bulk of this had been expelled from the tubes, and that the organisms were, moreover, found only in the silica lying at the bottom of the tubes, and therefore far away from the surface of the fluid. 


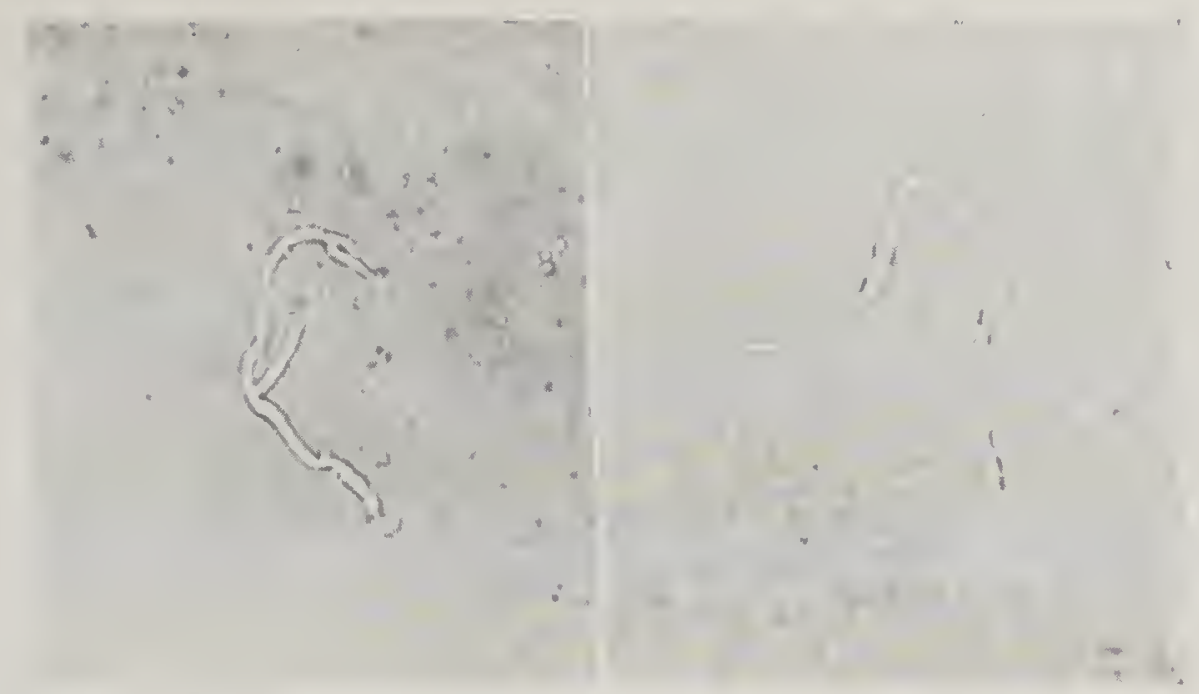

Fli. It ( $\times 500)$

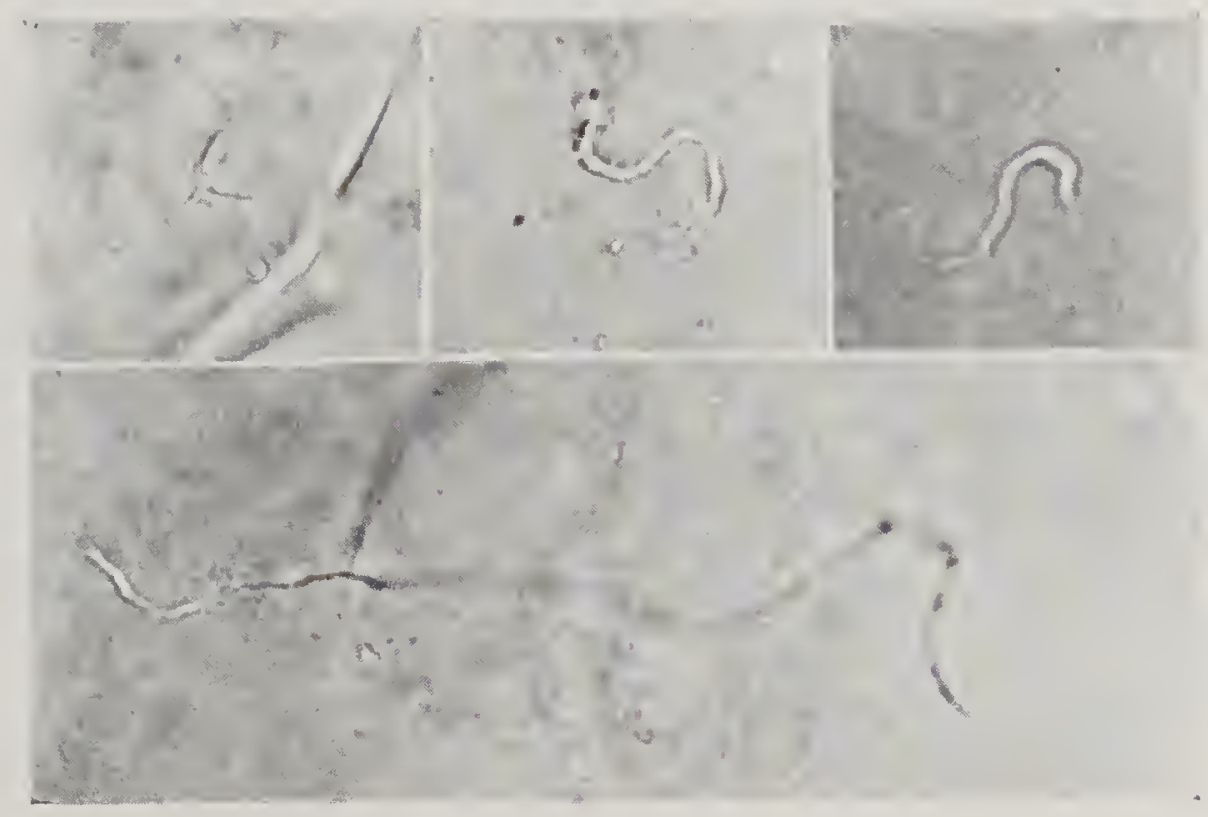

FIG. I5 $(\times 500)$

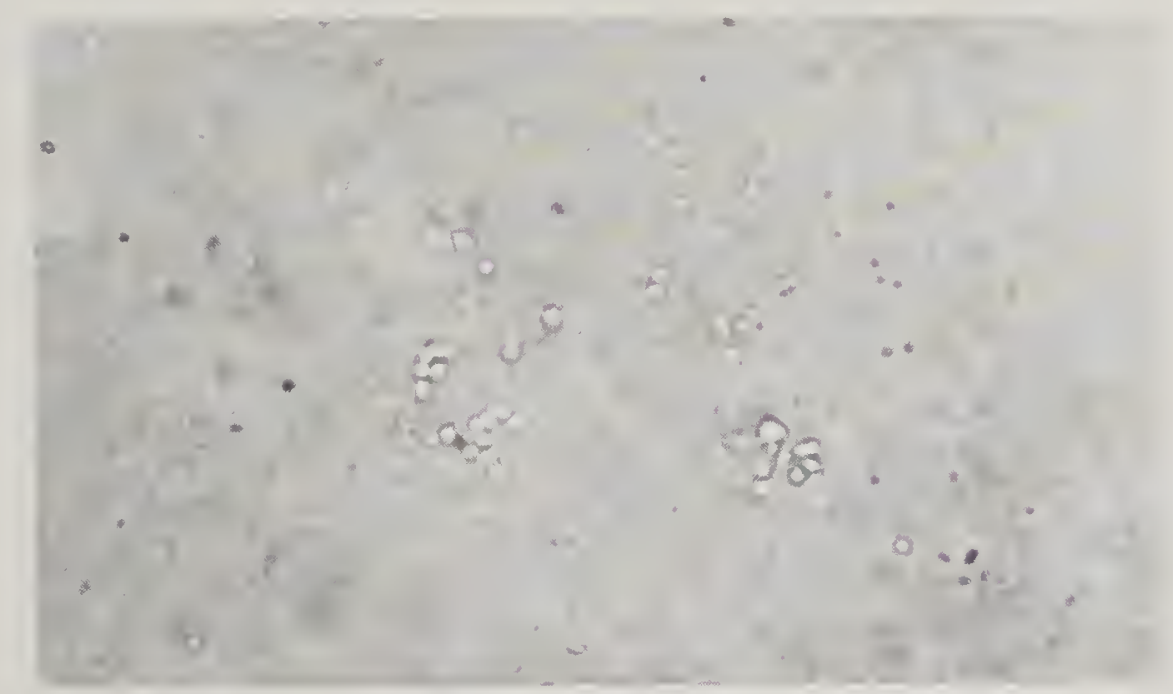

I.11:. $16(\times 700)$

OTHFR ORGANISAS TAKFN FROM PREVIOUSIS CLOSEI "TUPBS WHICH HAD IBEFN HEATEO TO MOK C. FOR TIN MINUTES 



\section{CHAPTER XX}

FINAL DECISIVE EXPERIMENTS

A LTHOUGH the fluids in the experiments A recorded in the last chapter were only heated to $100^{\circ} \mathrm{C}$. for ten minutes, we must not forget what the bacteriologist tells us concerning the potency of such a temperature for the destruction of Bacterial life. A few quotations will make this plain.

W. H. Park in his "Pathogenic Organisms" (1906, p. 451) says: "Whenever water is suspected, and there is any doubt as to the filters, it should be boiled for ten minutes; this will destroy all bacteria. This precaution should always be taken in the presence of typhoid fever and Cholera Epidemics."

George Newman in "Bacteria" (I899, p. 8I) says: "There is but one perfectly reliable method of sterilising water for household use, viz. boiling. As we have seen, moist heat at the boiling-point maintained for five minutes will kill all bacteria and their spores."

Thresh and Porter, again, in "Preservatives in Food and Food Examination" (I906, p. I), say: "Nearly all bacteria are killed when submitted to a temperature of about $65^{\circ} \mathrm{C}$. for a short time, although their spores are not destroyed 
unless heated to $100^{\circ}$ C. or over for a few minutes."

It must be borne in mind that in the experiments last recorded we were dealing with sterilised tubes containing distilled water, and two or three chemical ingredients practically free from micro-organisms, yet after the sealed tubes had been exposed to the temperature of $100^{\circ} \mathrm{C}$. for ten minutes, and had subsequently been kept for a few weeks exposed either to diffuse daylight or to the heat of an incubator, they have been found, on examination, to contain multitudes of micro-organisms. The microorganisms, too, have been, in part, of such a kind (Torulæ and Cocci) as are well known to be killed at temperatures ranging from $60-75^{\circ} \mathrm{C}$., and although some Bacilli also have been present (known to be destroyed at similar temperatures), there is no reason whatever for supposing that the distilled water or either of the three chemicals employed would have contained the more resistant spores of Bacilli. These are, moreover, as I have shown, resistant only after a previous desiccation, and to a much more limited extent than is generally supposed.

No loophole for doubt must, however, be left. Although I have used freshly distilled water, in which no one has yet found spores of Bacilli, let us deal with our problem as though this distilled water might contain such organisms and their spores as are to be met with in ordinary tap-waters, or even in hay infusions. We have seen that after prolonged researches on this subject the present sub-director of 
the Pasteur Institute has declared, in regard to these spores in all such fluids, that "A temperature of $115^{\circ}$ C. sterilises them completely and most rapidly." Our final trials must, therefore, be directed to ascertaining what results can be obtained with the different saline solutions, dealt with in the last chapter, when they have been exposed to temperatures ranging from II $5^{\circ}$ - I $30^{\circ} \mathrm{C}$.

Results obtained with Saline Solutions which had previously been heated in hermetically Sealed Tubes to II $5^{\circ}$ C. $\left(239^{\circ}\right.$ F.) for ten minutes.

The experiments now to be referred to have all been made with $\mathrm{A}$ and $\mathrm{AA}$ solutions. In these experiments, as well as in others in which higher temperatures have been employed, the sealed tubes have invariably been placed in the calcium chloride or the oil bath, when this was cold, so that the tubes and their contents have been heated gradually with the bath, till the desired temperature has been attained.

At the expiration of the ten minutes the tubes have been removed from the bath with a large wooden forceps, and allowed to cool. They have then been cleaned, labelled, and either placed in an incubator at $95^{\circ} \mathrm{F}$., or exposed to diffuse daylight at a temperature which has sometimes been $35^{\circ}$, or even $40^{\circ} \mathrm{F}$., lower than this. Yet, at the expiration of five or six weeks, when the contents of the tubes were examined, living organisms have been found in those tubes exposed to the latter 
conditions, to be quite as numerous, sometimes more numerous, than in those that have been exposed in the dark to the much higher temperature of the incubator.

In all these solutions the supernatant fluid above the deposited silica remained perfectly clear. When the end of the tube had been cut off at a level sufficient to admit the passage of a wide-mouthed pipette, the latter (after sterilisation) was introduced into the tube, so as to remove some of the flakes of silica, and deposit them on a clean microscope slip for examination.

In a flake taken from one of these tubes that had been exposed to light for five weeks, there is shown in Plate VI., Fig. I7, A, one of numerous small aggregates of Bacteria that were seen scattered through it. Around these aggregates were multitudes of solitary Bacteria and Micrococci, together with a comparatively small number of obviously inorganic particles or concretions. A few short chains closely resembling Streptococci were also seen.

In Fig. I7, B, a portion of a large aggregate of Bacteria and Micrococci is shown (under a lower magnification) which, with others, was found in a similar tube except that it was composed of uviol glass. Solitary Bacteria, and also small groups of them, were scattered through the flakes, while the obvious inorganic concretions were very much more numerous than they were in the tube previously examined, though it had been prepared at the same time, and had been similarly exposed to light for five 



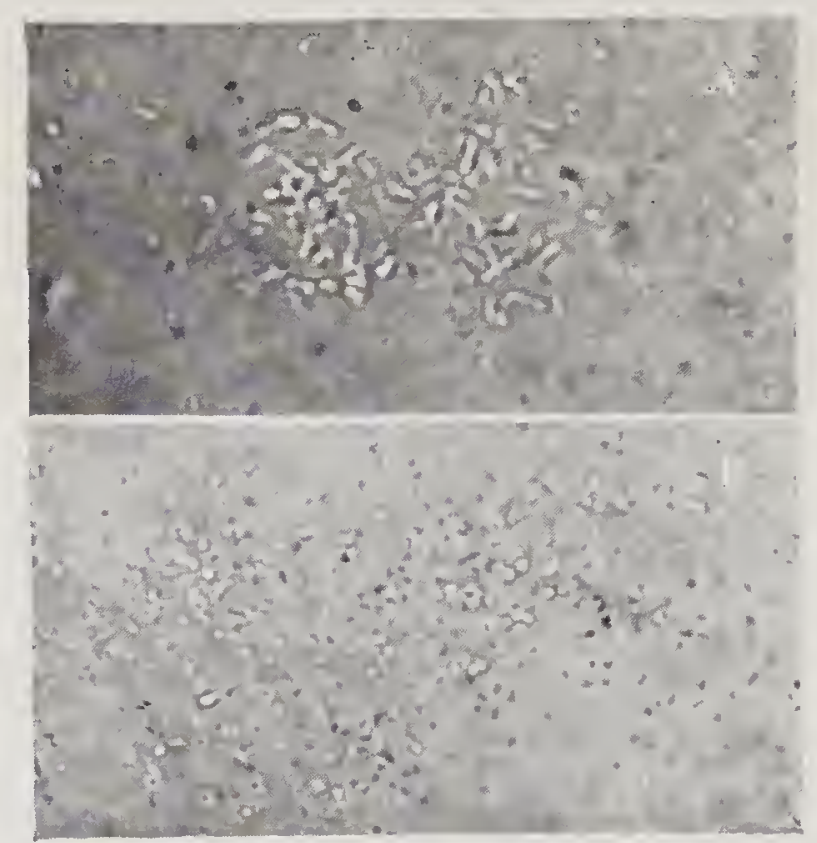

$116.17(\times 700.1 \times 1) 560)$

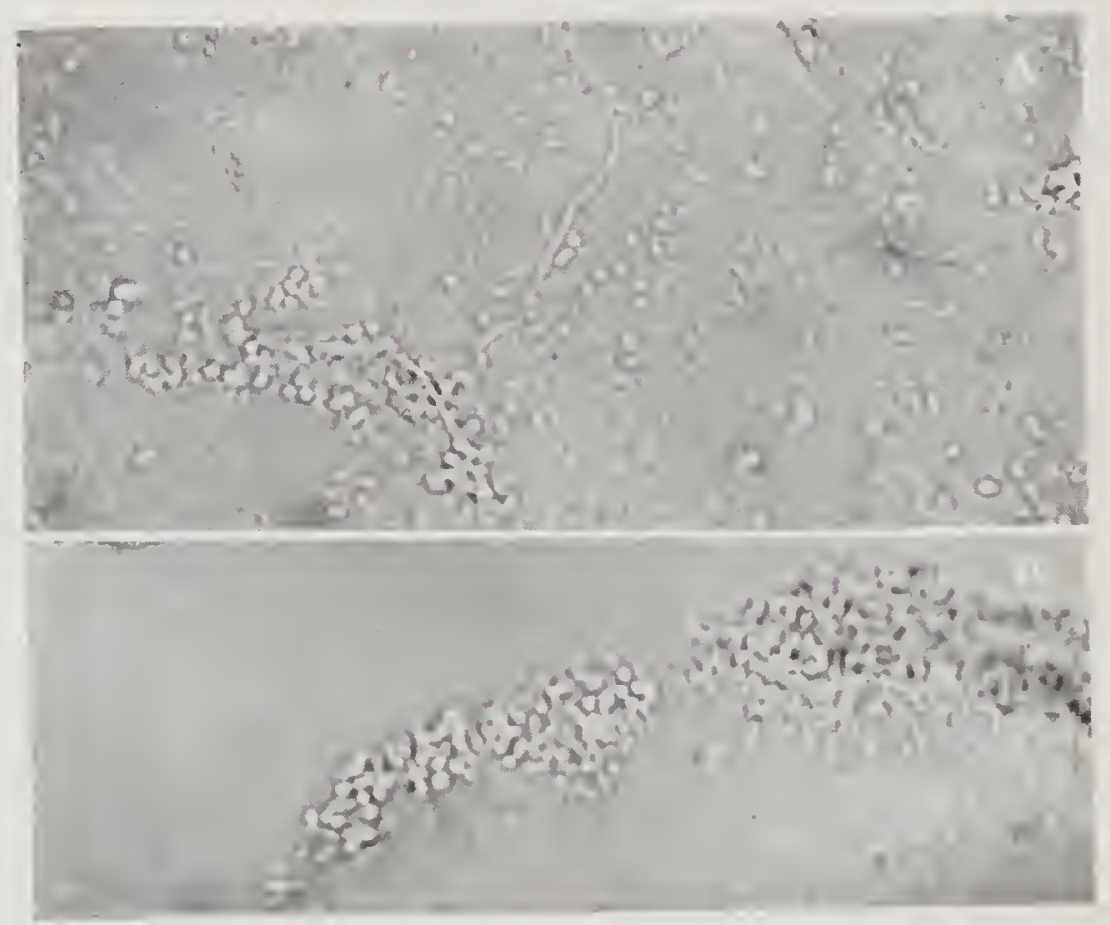

1.11. $18(\times 700)$

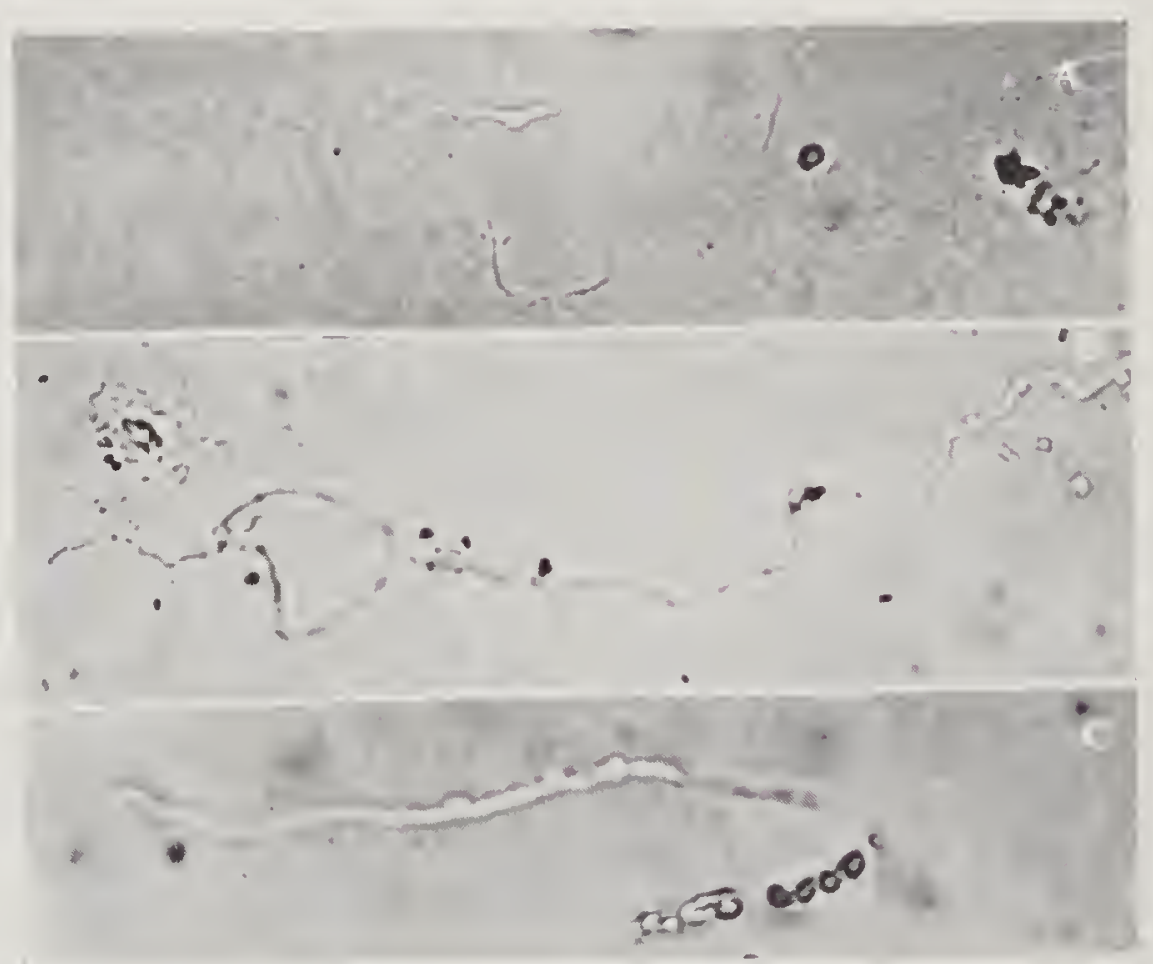

H.16. $19($ ) 500 iN1) $7(x))$

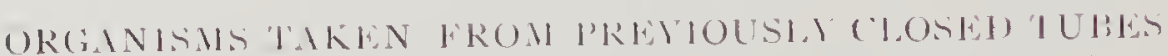

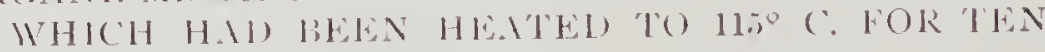
MINUTES 
weeks. No Streptococci, Torulæ, or incipient Moulds were seen in the deposit from this tube, though a number of long and much twisted Bacilli were found.

Both these tubes contained the A solution, while the two to which I am now about to refer were charged with the AA solution and, instead of being exposed to light, were kept in the dark incubator for five weeks. In and on the flakes of silica taken from one of them multitudes of minute Bacteria, mixed with a sparing number of long Bacilli, were found, such as are shown in Fig. I 8, A; while here and there, on the edges of some of the flakes, aggregates of Micrococci were found, like those that are to be seen in Fig. I8, B. A few incipient Moulds were also found, though these were more numerous in a companion tube, similarly charged and exposed, to which I am about to refer.

Incipient Moulds have already been shown in Figs. I4 and I5, taken from solutions that had been heated to $100^{\circ} \mathrm{C}$. only, and subsequently exposed to light; and now we shall meet with very similar bodies, taken from tubes heated to higher temperatures, and exposed either to heat or to light.

In Plate VI., Fig. I9, A, a very early stage of three of these Moulds is to be seen, while in B a part of a much more developed specimen is shown. The tapering nature of the extremity on the left may be recognised, while that on the right (not shown in the photograph) was very similar. In Fig. I9, C, a more highly magnified specimen is to 
be seen, and the Mould is shown to be commencing from a group of refractive particles, very similar to what are to be seen in the mid-portions of Fig. 2 I in the next plate. Such particles may be seen also in Plate V., Fig. I 5, A and B, in the substance of the incipient mycelia there represented.

Results obtained with Saline Solutions which had previously been heated in hermetically sealed Tubes to I $20^{\circ}$ C. $\left(248^{\circ} \mathrm{F}.\right)$ for ten minutes.

One of the tubes charged with some of the AA solution that had been heated as above indicated, was examined after it had been in the incubator for six weeks. Numerous groups of Bacteria and Micrococci were found in and on the flakes of silica, both in small and in rather large aggregates. Incipient Moulds were also found, though they were rather scarce. A portion of one of the large aggregates of Bacteria is shown in Plate VII., Fig. 20, and one of the Moulds in Fig: $2 \mathrm{I}$. This latter was rather a remarkable specimen. It is shown in A under a low magnification, in order that both the filiform extremities may be seen; while in $\mathrm{B}$ the greatly expanded median portion, containing many refractive particles, is better seen, since it has been more highly magnified.

Another tube, charged at the same time with some of the same solution and heated in the same bath, was exposed to light rather than placed in the incubator. Its contents were also examined at the expiration of six weeks, and in the first flakes of 


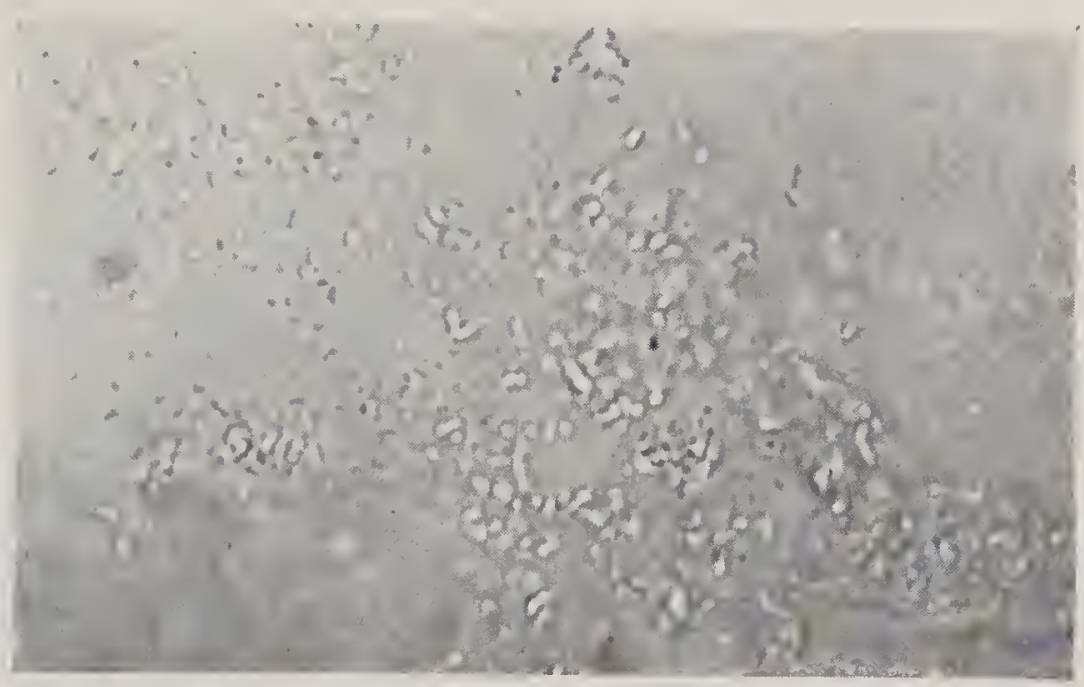

Fici. $20(\times 700)$

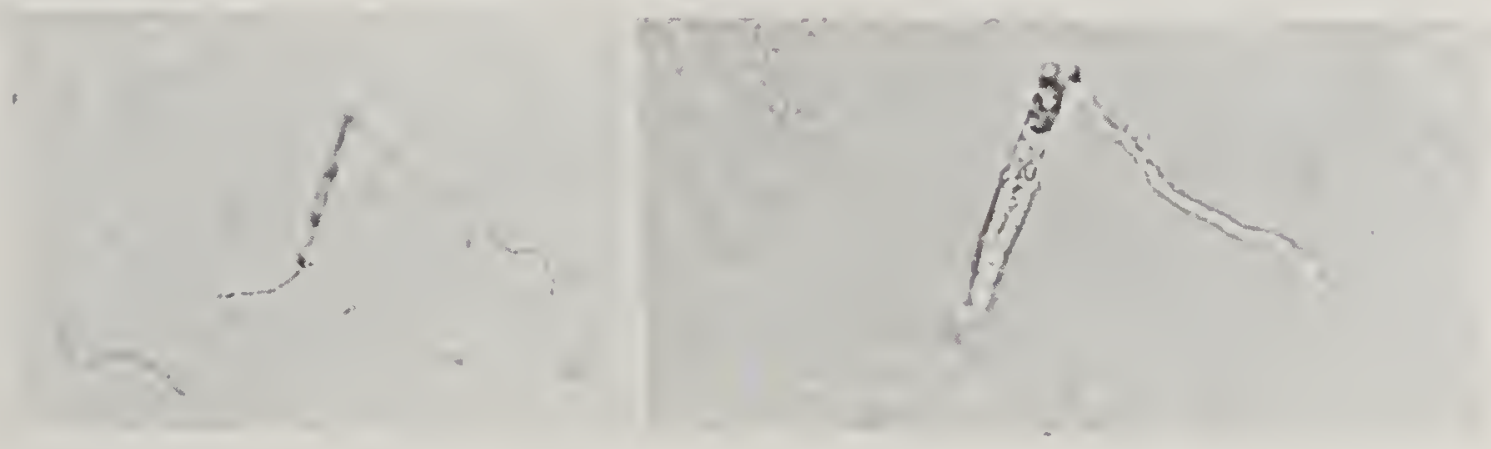

FICi. $2 I(\times 250$ AND 500$)$

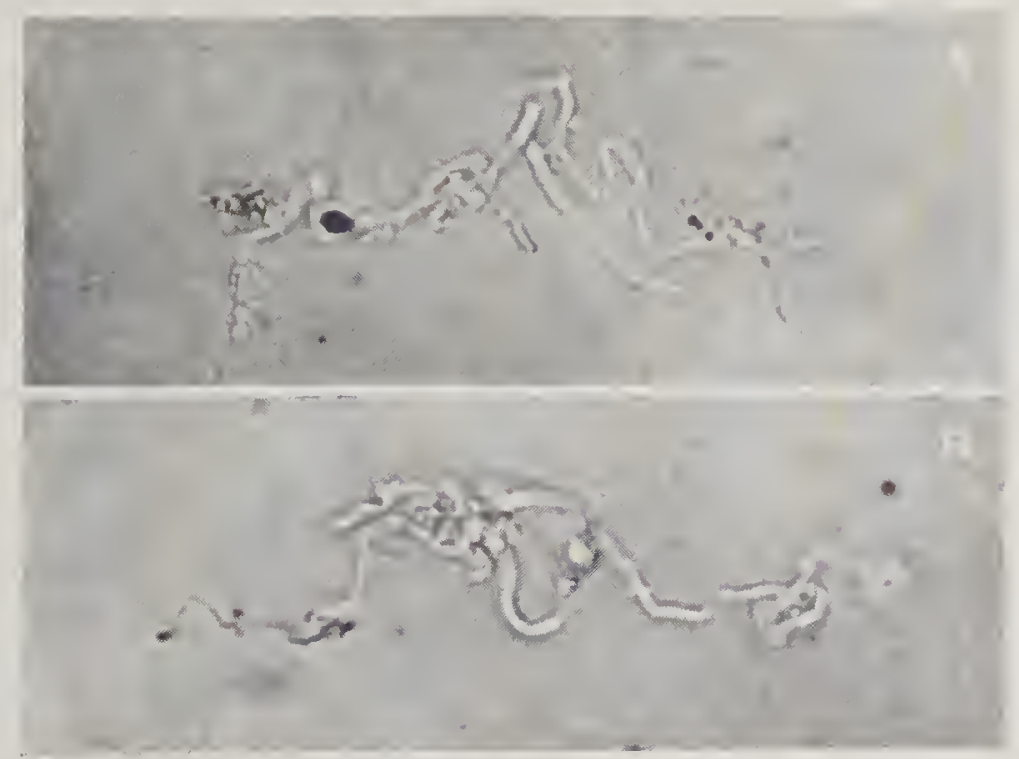

Lifi. $22(x ; 00)$

ORGINISMG TAKEN FROM PRUVIOUSJY CIOSED TUPES WHICH HAI) BWEN HEATED TO $120^{\circ}$ (. FOR TEN MINUTES 

silica abstracted from the tube more numerous, as well as longer, groups of Bacteria were seen, and also several incipient Moulds, whose hyphæ were twisted and slightly branched, such as are shown in Fig. 22, A and B. These small Moulds were distinctly more numerous, and much more developed in this tube which had been exposed to light than were those taken from the companion tube, that had been in the incubator, and quite $35^{\circ} \mathrm{F}$. hotter.

Results obtained with Saline Solutions which had previously been heated in hermetically sealed Tubes to $125^{\circ}$ C. $\left(257^{\circ} \mathrm{F}\right.$.) for ten mimutes.

One of the tubes with the A solution, and heated as above indicated, was exposed to light for five weeks, when it was opened and some of the flakes of silica were withdrawn with a sterilised pipette. They were found to be crowded with Bacteria, isolated and in small aggregates, one of the latter of which is shown in Plate VIII., Fig. 23. The inorganic concretions distributed through the flakes were numerous, but quite small and often impossible to be certainly discriminated from isolated Bacteria or Micrococci. No Torulæ or incipient Moulds were found.

A companion tube prepared at the same time with some of the same solution, similarly heated and similarly exposed to light for five weeks, was also examined. The flakes of silica were found to be absolutely crowded with very large inorganic concretions such as are shown in Plate XII., 
Fig. 38 , and no living thing of any kind could be discovered in any of the flakes examined. The only known cause of this remarkable difference was the fact that this A solution had been heated in a tube of uviol glass, and had subsequently been exposed to light therein. The combined result was this remarkable change in the flakes of silica, and the nonappearance of any living thing.

An ordinary tube that had been charged with the AA solution and heated as above, was exposed to light for six weeks. On examination a large amount of silica was found to have been deposited, and the flakes were large. They were found to contain comparatively few of the organic particles, and those that existed were small. Small groups of Bacteria were seen, some of them with what appeared to be a Torula corpuscle in their midst, as shown in Plate VIII., Fig. 24, A, similar to what had been found in some of the earlier tentative experiments (see Plate II., Fig. 5). A few separate Torulæ were also seen, one of which, as shown in Fig. 25, A, was germinating.

The inorganic particles were far less abundant in this AA solution in common glass than they were in the A solution above referred to, in which all the conditions were similar except that one solution contained rather more silica than the other. These facts, as well as that above referred to in reference to the extraordinary change produced in the A solution when heated in and exposed to light in a uviol tube, are important as showing the different effects produced by high temperatures upon the 


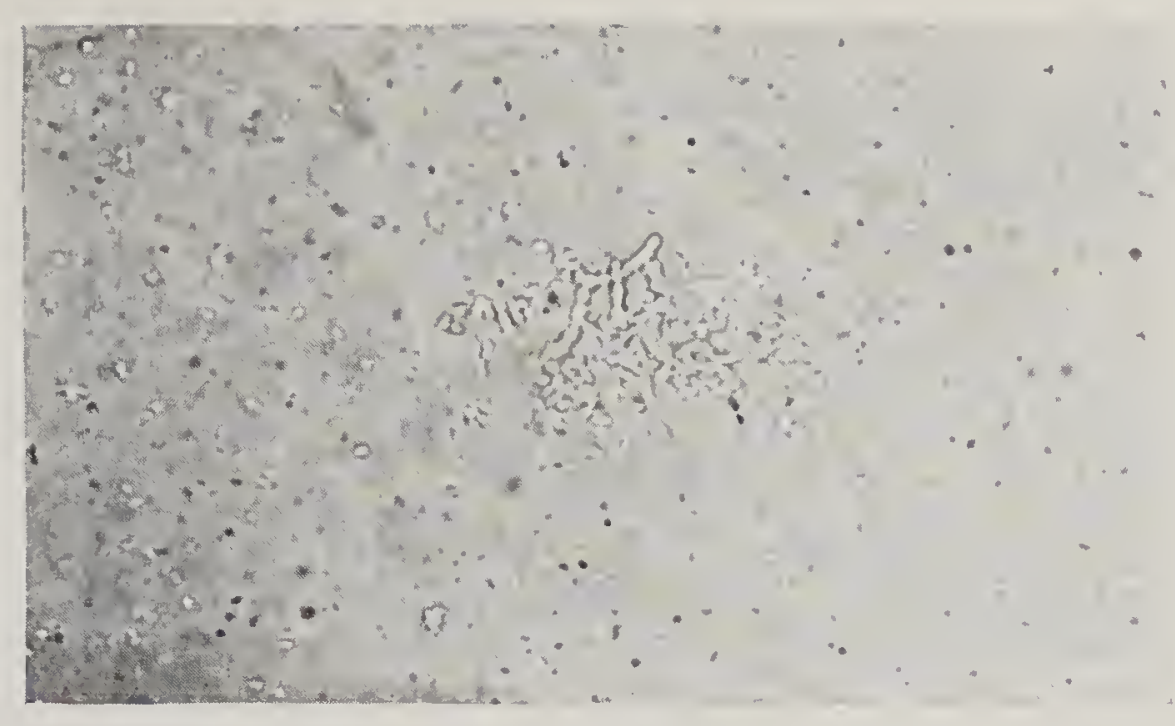

FIG $23(x 500)$

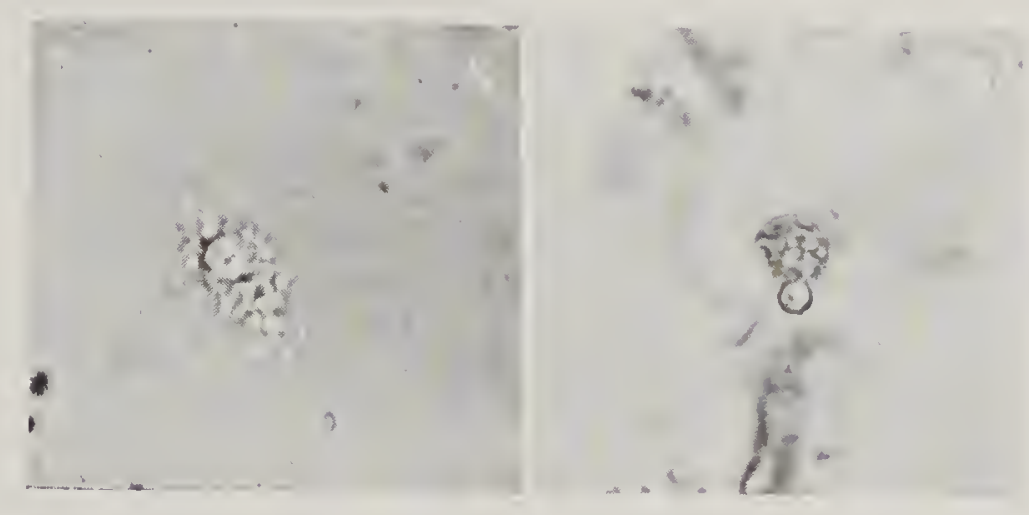

FIG. $24(\times 700$ AND 500$)$

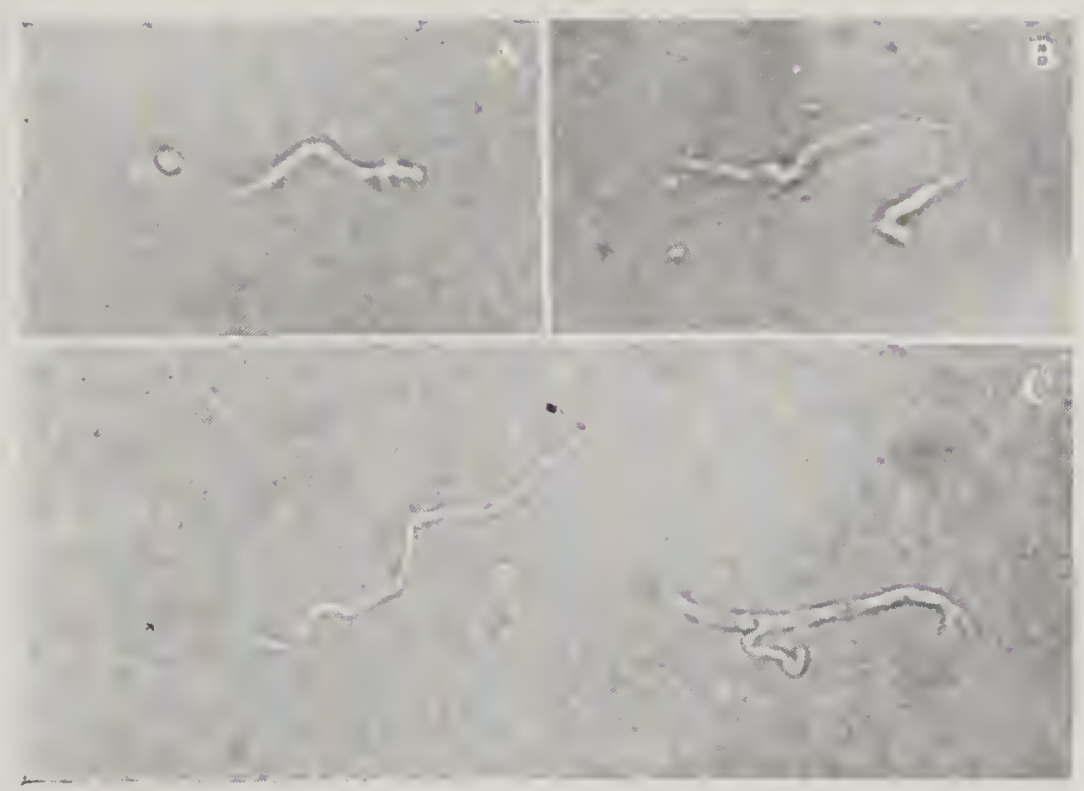

FIG. $25(\times 700$ AND 500$)$

ORGANISMS TAKEN FRON PREVIOUSIA CIOSEM TULES WHICH H.AI ISEN HEATEA TO 1250 C. FOR THN MIUTES 

media themselves, when they are slightly different in constitution or have been exposed to slightly different conditions. See also p. 274 and Pl. XII. in reference to this point.

In another tube charged with the AA solution which, after superheating, had been kept in the incubator for six weeks, there was a similar paucity of inorganic concretions distributed through the flakes. Bacteria were also very scarce, and only a few incipient Moulds were seen. One of these is represented in Fig. 25, B, while another much more developed specimen is shown in $\mathrm{C}$, a portion of which (to the right of the centre) being invisible owing to its being below the proper focus.

In two specimens of the $B$ solution which had been heated to $125^{\circ} \mathrm{C}$. for ten minutes, and subsequently kept in the incubator for five weeks, no distinct organisms were found, though there were a number of bodies of uncertain nature. One of these is shown in Fig. 24, B. Another exactly like it was seen, together with many smaller bodies of like kind, of different sizes, without the bud-like outgrowth or projection.

Results obtained with Saline Solutions which had previously been heated in hermetically sealed Tubes to $130^{\circ} \mathrm{C}$. $\left(266^{\circ} \mathrm{F}\right.$.) for twenty mimutes.

Two tubes having been charged with the AA solution, were exposed to light for the first three months, and after that were placed in the incubator. The contents of one of them was 
examined after the tube had been in the incubator for one month. There were many cocci-like particles in the flakes which may have been all inorganic, as no Bacilli or distinct aggregates of Bacteria were seen. A very few Torulæ, either single or in small groups, were found, but there were a number of long bent filaments, such as are shown in Plate IX., Fig. 26. They had in places, and when more highly magnified, an appearance suggestive of Streptococcal threads, though they were more like what Lehmann and Neumann in their "Principles of Bacteriology" describe as "Torula threads" (loc. cit. Fig. I, r).

I determined to leave the companion tube in the incubator for another six weeks, and then, on examination of its contents, these peculiar threads were found to be not only much more numerous, but also here and there aggregated into much larger masses, one of which is shown in Fig. 27. There had, therefore, been a considerable growth and development of these threads during the extra time in which the tube had been allowed to remain in the incubator. There were also a few bodies looking like Torula, either solitary or with a single bud, and mostly showing a nuclear particle. Still none of them were vegretating, and there were no groups. I was not sure, therefore, that they might not be inorganic products.

These tubes chargred with the AA solution, which had been heated to a higher temperature, and had been maintained at that temperature also for a longer period than in any of the other experiments 


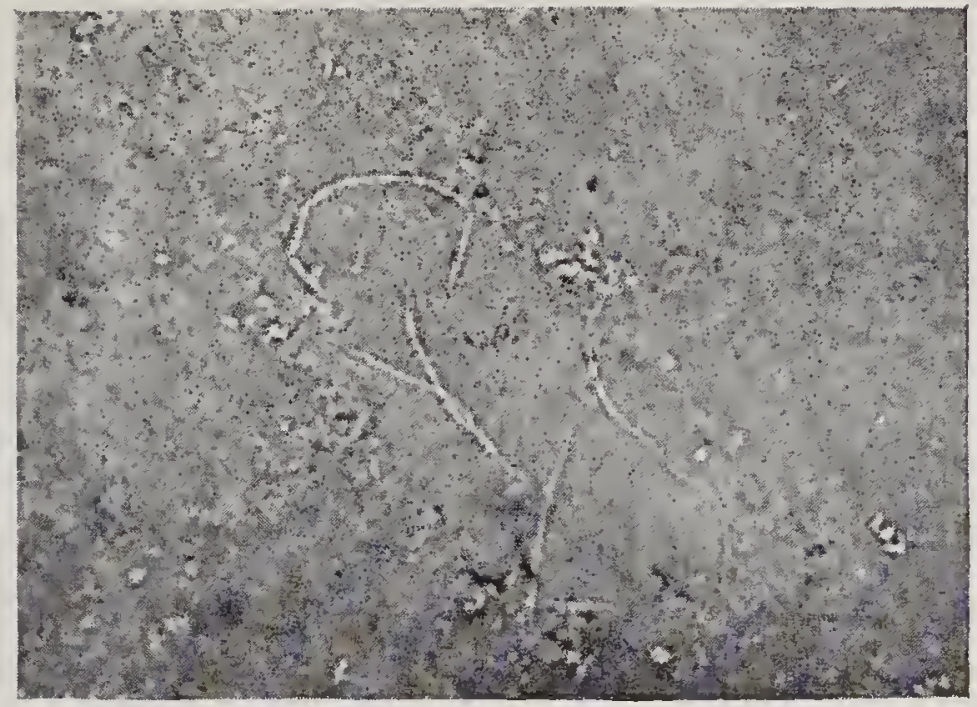

FIG. $26(\times 500)$

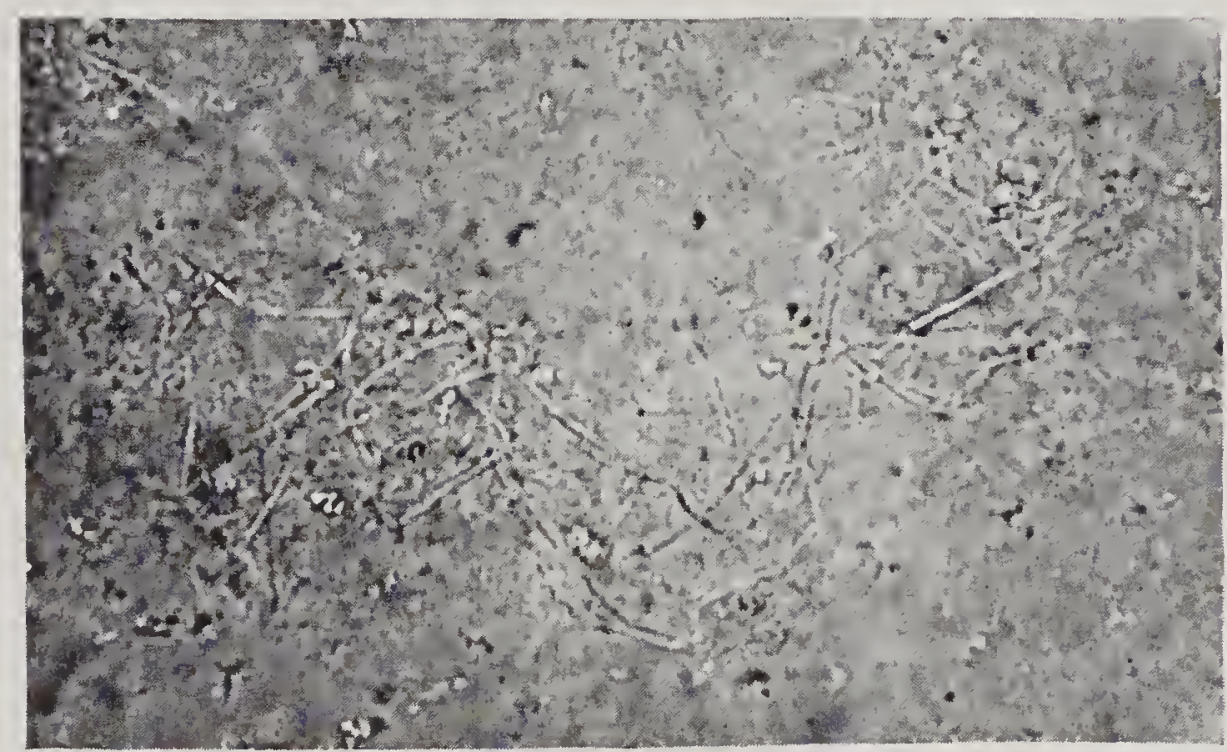

FIG. $27(\times 570)$

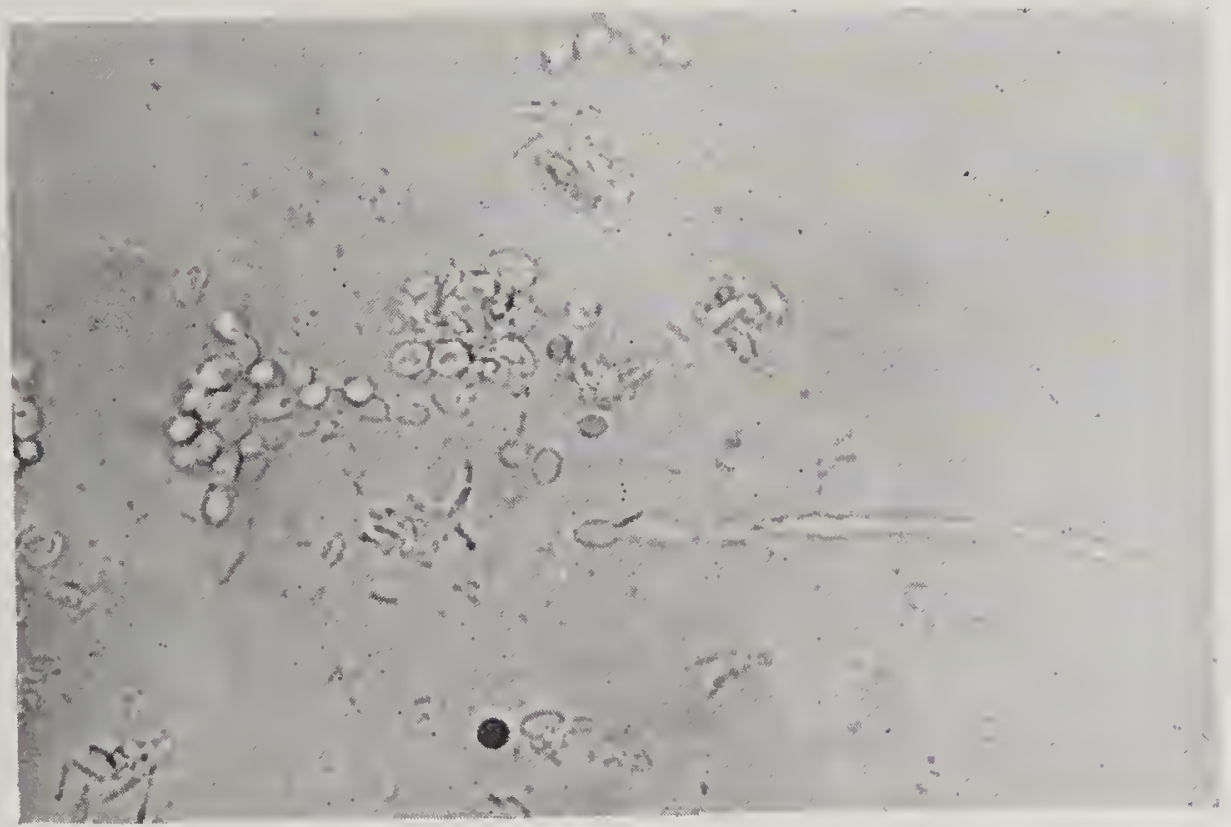

FIG. $28(\times 700)$

ORGANISIS TAKEN FROM PREVIOUSLY CLOSED TUBES WHICH HAD IEEN HEATEI TO $130^{\circ} \mathrm{C}$. FOR TWENTY MINUTES 

recorded in this Chapter, were purposely left exposed to light for a longer period, and subsequently placed in the incubator; because several other tubes containing such a solution, that had been similarly heated to $130^{\circ}$ for $20^{\prime}$, on examination, after shorter periods of exposure, had been found to be barren.

These particular superheated tubes had been examined earlier, because in the first experiment I performed this year with similar chemicals similarly heated (though in slightly different proportions), the result had been very successful. On opening this tube after seven and a half weeks and examining some of the flakes of silica, plenty of Bacteria were found, and also a number of delicate Torulæ, both solitary and in groups, such as are shown in Fig. 28. Unfortunately I do not know the exact proportion of the different ingredients employed in this preliminary trial with such a solution heated to I $30^{\circ} \mathrm{C}$. for $20^{\prime}$, and subsequently exposed to light. But in no experiment since performed with an AA solution heated to such a temperature have I been able to obtain similarly successful results, as far as Bacteria and Torulæ are concerned.

I now have to refer to some remarkable experiments in which the $\mathrm{BB}$ solution, or a slight variation of it, has been employed; and in which longer periods of exposure to light and heat have been generally had recourse to.

In the first two of these experiments to which I shall refer there were six rather than eight drops of 
the liquor ferri pernitratis to the ounce of distilled water (together with six drops of the sodium silicate solution). In the first experiment the tube was of soft German glass, while in the second a uviol tube was employed.

The sediment in the first tube was of a pale yellowish colour, and the fluid above was of the same tint and slightly opalescent. When opened, after an exposure for twelve weeks to diffuse daylight, the fluid was found to be slightly acid. On examination of some of the sediment three small mycelia were found, with rather broad hyphæ. No unmistakable Torule or Micrococci were seen, but there were a large number of Vibriones in many places, thickly scattered through the substance of the small granular flakes of which the sediment was largely composed. Some of these bodies, slightly stained with gentian violet, are shown in Plate X., Fig. 29, A, while in B unstained specimens are to be more dimly seen diffused through the substance of a granular flake. These bodies were almost exactly like those shown in Plate IV., Figs. 12 and 13.

The tube was closed after two specimens had been taken from it with a sterilised pipette, and was then left in a box for five weeks, when its contents were again examined. There seemed to be a distinct increase in the number of the Vibriones, but no other change was recognised, so the tube was not kept longer.

After the uviol tube had been exposed to light for twelve weeks, it was placed in the incubator for another two weeks, and was then opened. The 


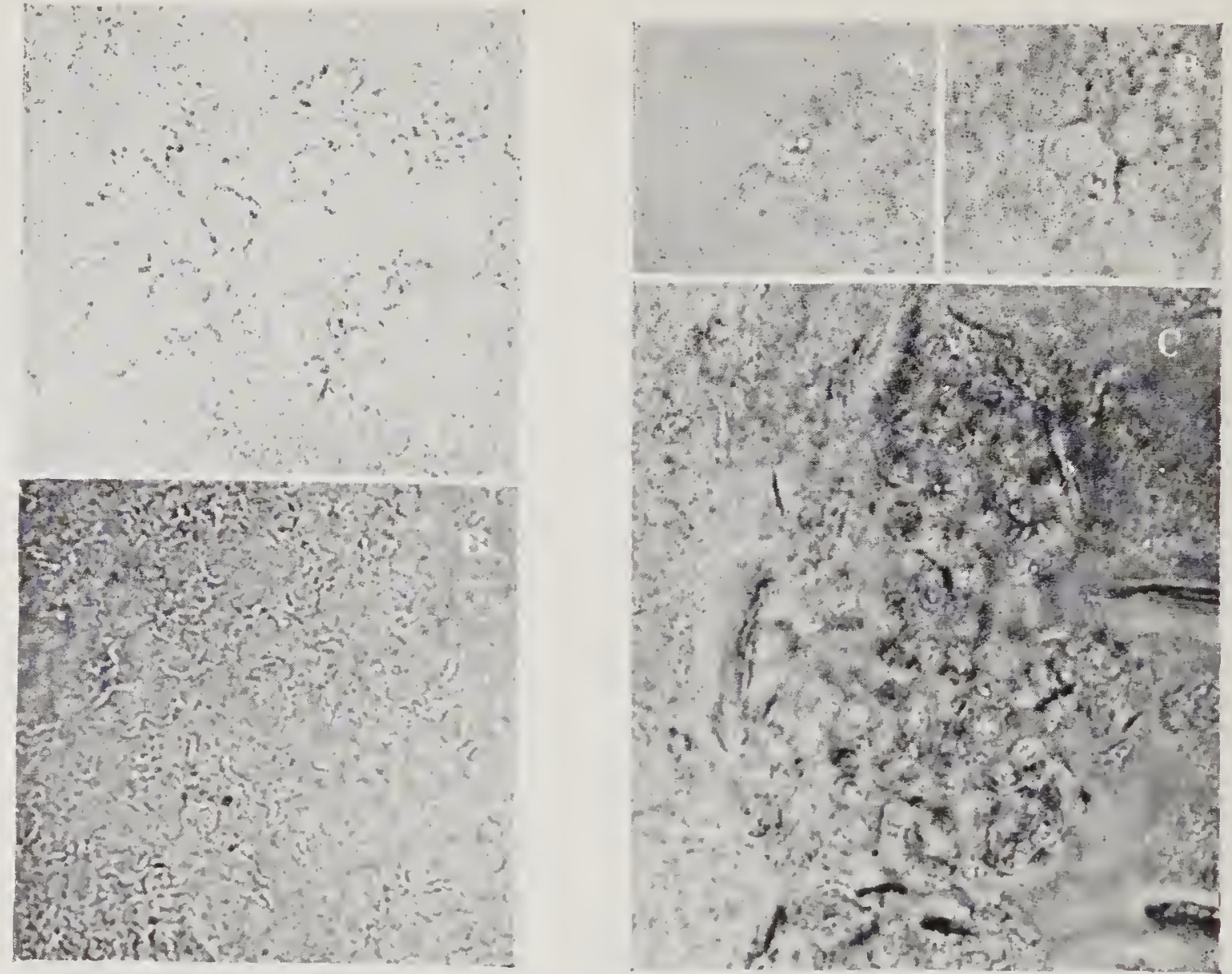

FIG. $29(\times 700$ AND 500)

FIG. $30(\times 700$ A.II $=00)$
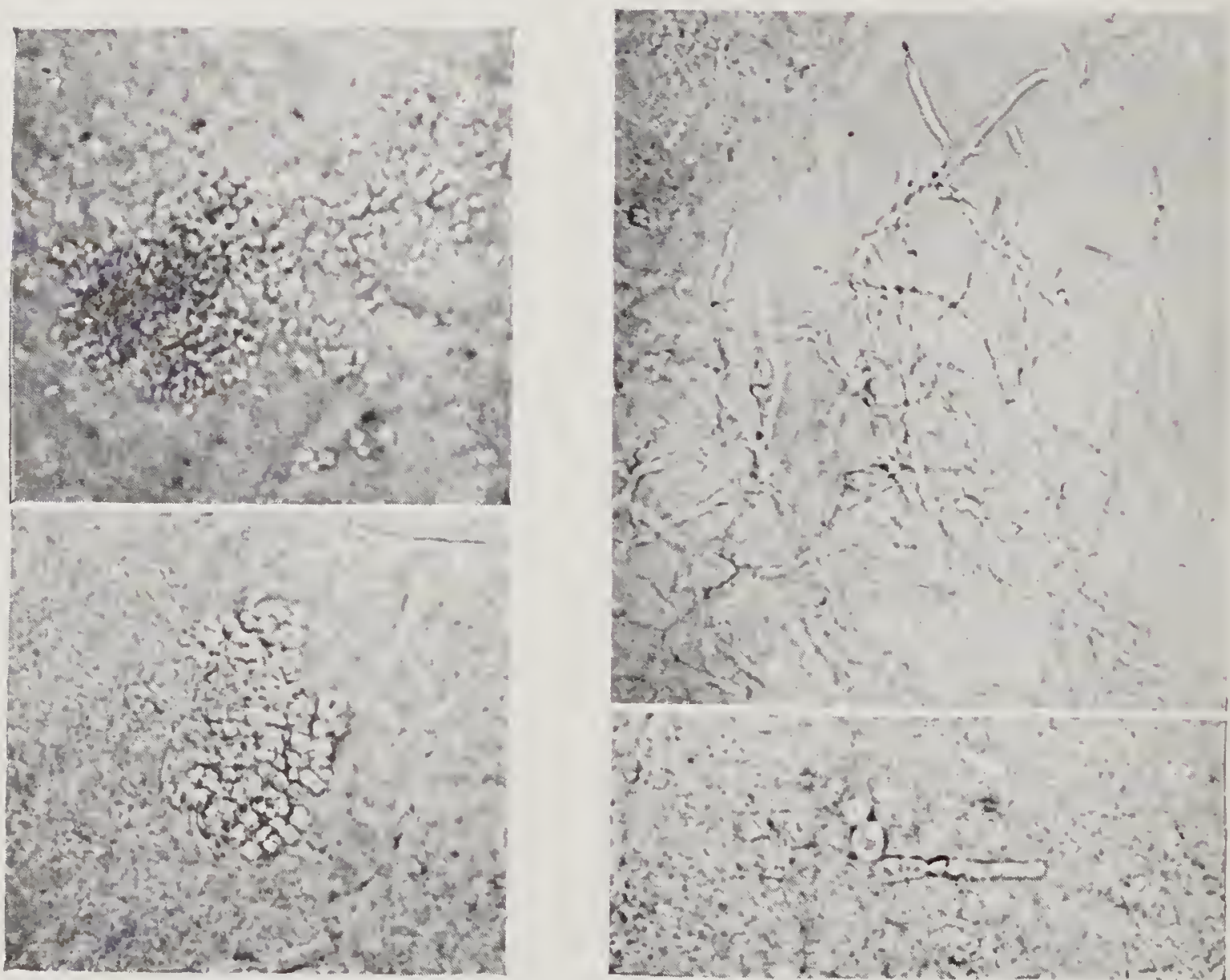

FIG. $31(X 700$ ANI 500$)$

FIfi. $32(\times 500)$

O'THER OR(DNISMS TAKEN FROM PREVIOUSLY CIOSEI) TUBES WHICH HAI) LEEN HEATED TO $130^{\circ}$ C. FOR TWENTY MINUTES 
fluid and sediment were similar to the last in appearance, but the reaction of the fluid was neutral. On microscopical examination of some of the sediment a single small incipient Mould was found, while Vibriones, as in the last tube, were fairly numerous in the substance of the granular flakes. No other organisms were found, and the tube was not kept longer for further observation.

In the next two experiments to be referred to the ordinary BB solution was used. Both tubes were of the ordinary German glass. The fluid and sediment presented the same appearance as before. One of the tubes was opened after it had been exposed for twelve weeks to daylight during very hot weather (from June Ist to August 25th). The reaction of the fluid was faintly acid, and in the first two samples of the sediment examined I found many aggregates of Vibriones such as are shown in Fig. 29, and also many groups of Micrococci, both free and loculated. From some of the groups of free Micrococci filaments, either straight or twisted, were seen growing; while the loculated Cocci, the early stages of which are shown in Fig. 30, A and B, were almost exactly like those represented in Plate III., Figs. 8, 9, and 10, from a similar solution that had been heated only to $100^{\circ} \mathrm{C}$. in a corked flask. One very large sponge-like mass of such loculated Micrococci was also found embedded in, and somewhat obscured by, the substance of a granular flake. This is represented, under a lower power, in Fig. 30, C. In the lower right-hand border its constituent groups of Micrococci can be best made out. 
After these two samples of the sediment had been abstracted with a sterilised pipette, the tube was again closed and put away in a box (at the ordinary temperature of the air) for another eleven weeks. ${ }^{1}$ When opened on November I 2 th the fluid was found to be neutral rather than slightly acid, and was swarming with organisms of different kinds. There was a great increase in the number of the Vibriones, and also of the Micrococci both free and loculated. One of the large groups of the former is shown in Fig. 3 I, A, partly stained with eosin. There were also groups having all the appearance of Sarcinæ, one of which is shown in B. Lehmann and Neumann say (loc. cit. p. 15I): "It is our conviction that the Sarcina is connected with the Micrococci by unbroken transition forms," and the appearance of the groups of Sarcinæ here found certainly lent support to this view. Torulæ were also found, such as are represented in Fig. 32, B, together with one small mass of Mould about the size of a small pin's head, from which myriads of hyphæ were issuing in all directions, some of which, at the periphery of the mass, are shown in Fig. 32, A. The advantage of leaving tubes containing such a solution a very long time before they

1 In reference to what follows, it is well that certain facts concerning the paucity of micro-organisms in the air should be borne in mind, as recorded by an eminent bacteriologist, Dr Allan Macfadyen, in a Friday evening discourse at the Royal Institution in June 1900 (see Proceedings, vol. xvi. p. 45r). Speaking of a special investigation made by himself and Mr Lunt, he says: "In the open air of London there was on an average just one organism to every 33,300,000 dust particles present in the air; and in the air of a room, amongst $184,000,000$ dust particles, only one organism could be detected." 



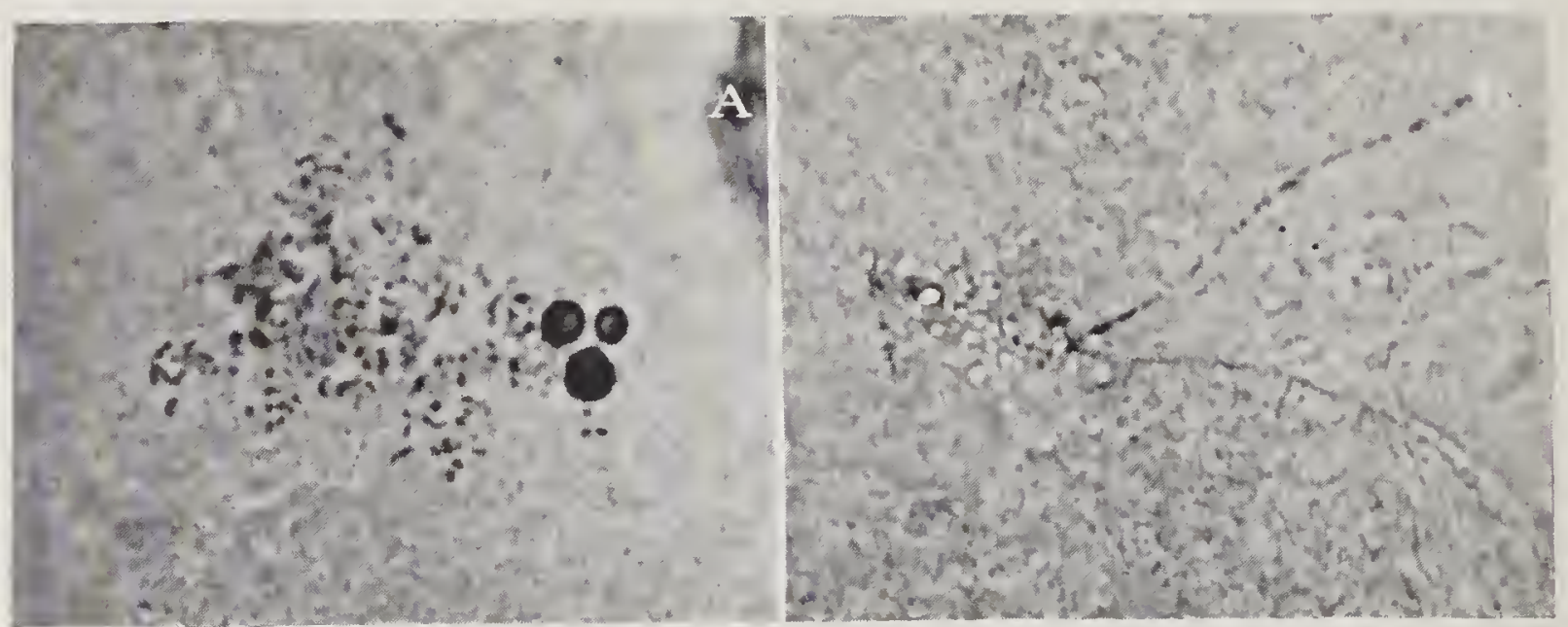

Flr: $33(\times 700)$

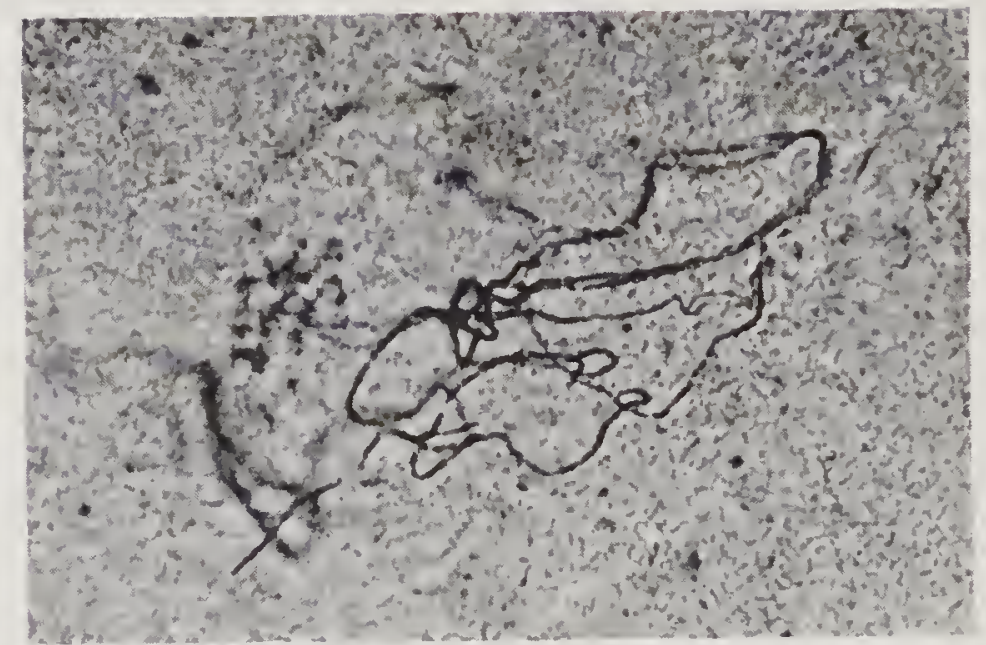

I.IC. $34(\times 500)$

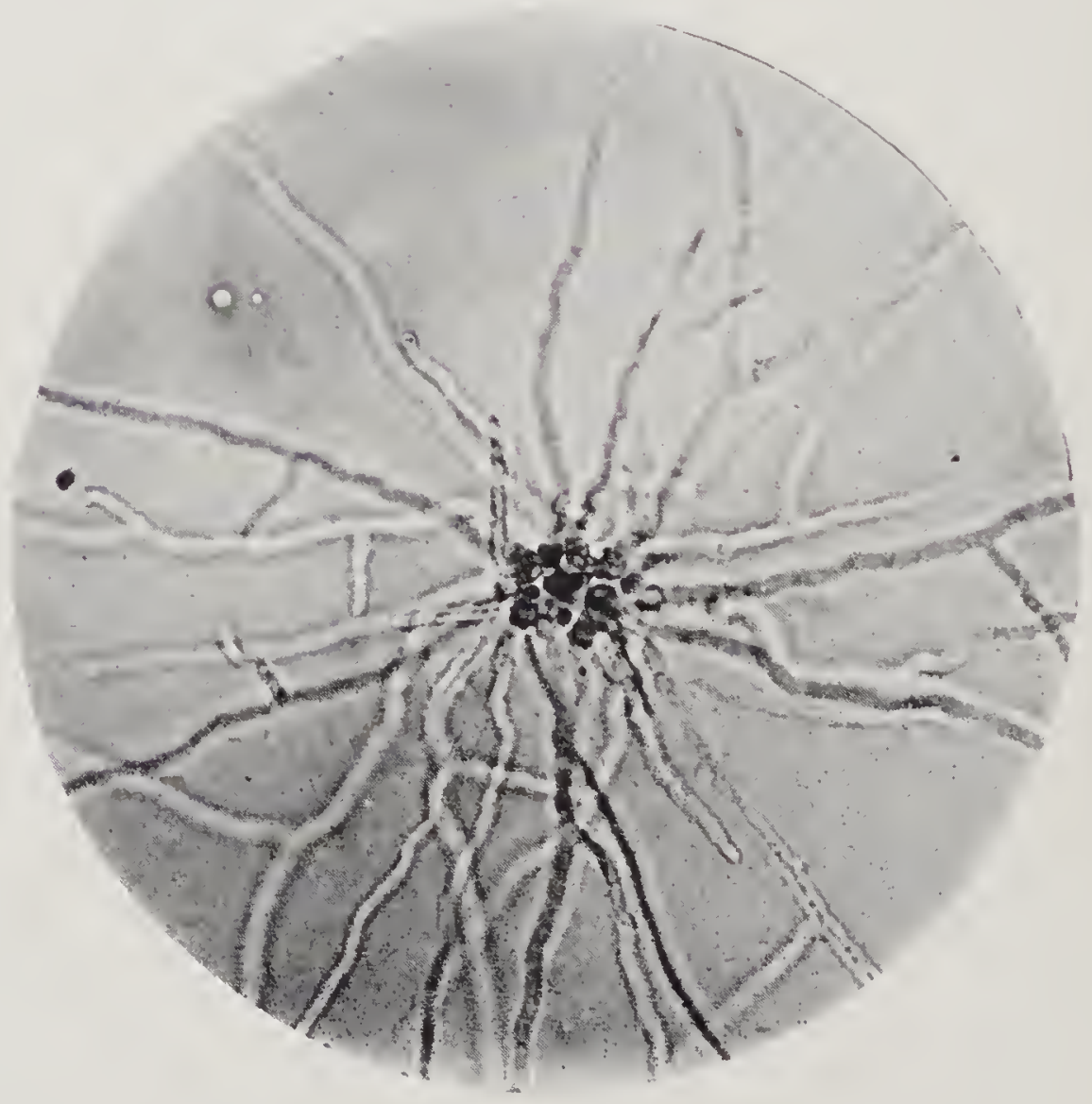

FIG. $35(\times 375)$

OTHER ORGANISMS T.IKEN FROM PREVIOUSLY CLOSED TUBES WHICH HAI) LEFA HEATEO TO $130^{\circ}$ C. HOR TWENTY MINUTES 
are opened was thus fully shown. It is impossible for me to believe that contamination of the fluid had occurred by the passage into it of the sterilised pipette, or from its brief exposure to air. The fluid itself remained free from organisms; these, as usual, being found only in and on the substance of the flakes of sediment, lying at the bottom of the tube.

A companion tube containing some of the same $\mathrm{BB}$ solution, which had been prepared and heated at the same time to $130^{\circ} \mathrm{C}$. for twenty minutes, was opened and its contents examined on July $\mathrm{I} 3^{\text {th }}$ - that is, after only six weeks. The first sample of the sediment taken from this tube with a sterilised pipette showed many groups of Micrococci, both free and loculated, and also some Torula corpuscles. A drop of eosin was slowly drawn beneath the cover glass, so as partly to stain the organisms, and subsequently several photographs were taken from this single specimen of the sediment. The tube itself was at once resealed, and subsequently left in a box for another six weeks at the temperature of the airwhich was mostly over $70^{\circ} \mathrm{F}$. for some weeks.

The loculated Micrococci found in the first drop were exactly like those shown in Figs. 30 and 9. One of the large groups of free Micrococci, together with three Torula cells, all stained of a deep red by the eosin, are shown in Plate XI., Fig. 33, A; while another group of free Micrococci, only a few of which are stained, is represented in B, from two of the units of which partly stained filaments are proceeding. From another small group of free Micrococci two much twisted filaments were seen issuing, 
as shown in Fig. 34. These filaments are only dimly seen at their origin from the Cocci, in the photograph, owing to their being, at this point, rather out of focus.

When this tube was opened and examined again, after the six weeks, very many groups of Torulæe were found in a specimen of the sediment taken therefrom, and from the groups of Torulæ many short hyphæ were issuing. Several good photographs were obtained of different groups after they had been stained with eosin, one of which is shown in Fig. 35. It seems clear that the Torulæ seen there, under a lower magnification, are just such bodies as were found when the tube was first opened (see Fig. 33, A, × 700). Some of the aggregates of Torulæ were small, but others were much larger, showing that they had multiplied considerably before beginning to throw out hyphæ. In a stained specimen-where the cover glass had been surrounded by paraffin-I found, when it was examined two days later, that some marked specimens of the Mould had grown in the interval, notwithstanding the eosin: the hypha being very distinctly longer than they were when first seen.

I have already alluded to the changes produced by high temperatures on different saline solutions (p. 266), and as a sequence it seems well to give some illustrations showing something of the effects of different degrees of heat upon flakes of silica taken from some of the A solution, and when exposed to light in tubes of different kinds. 



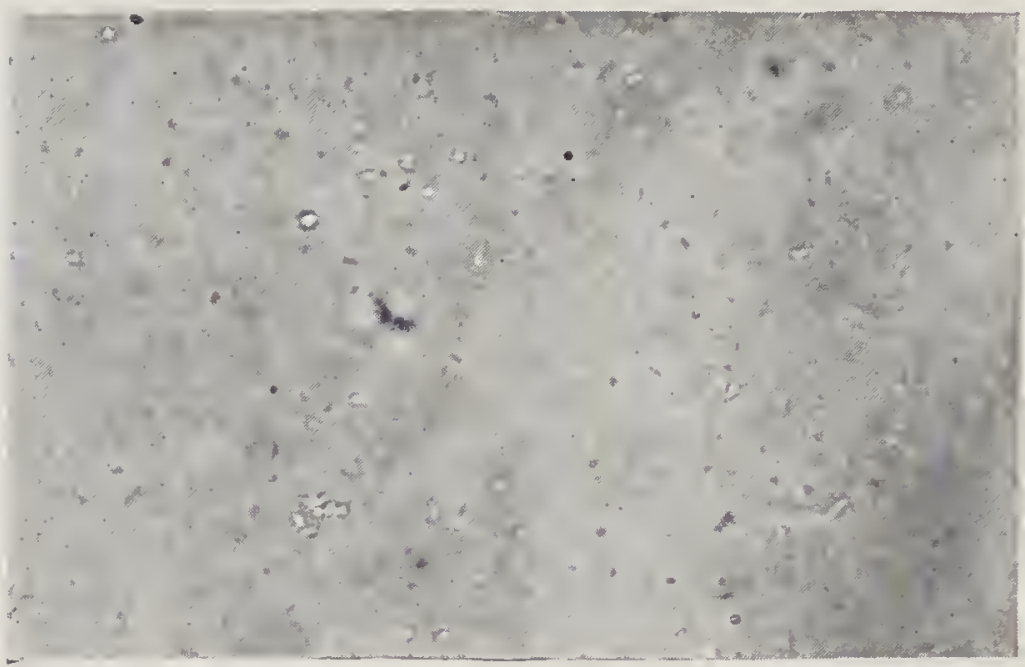

FIG. $36(\times 500)$

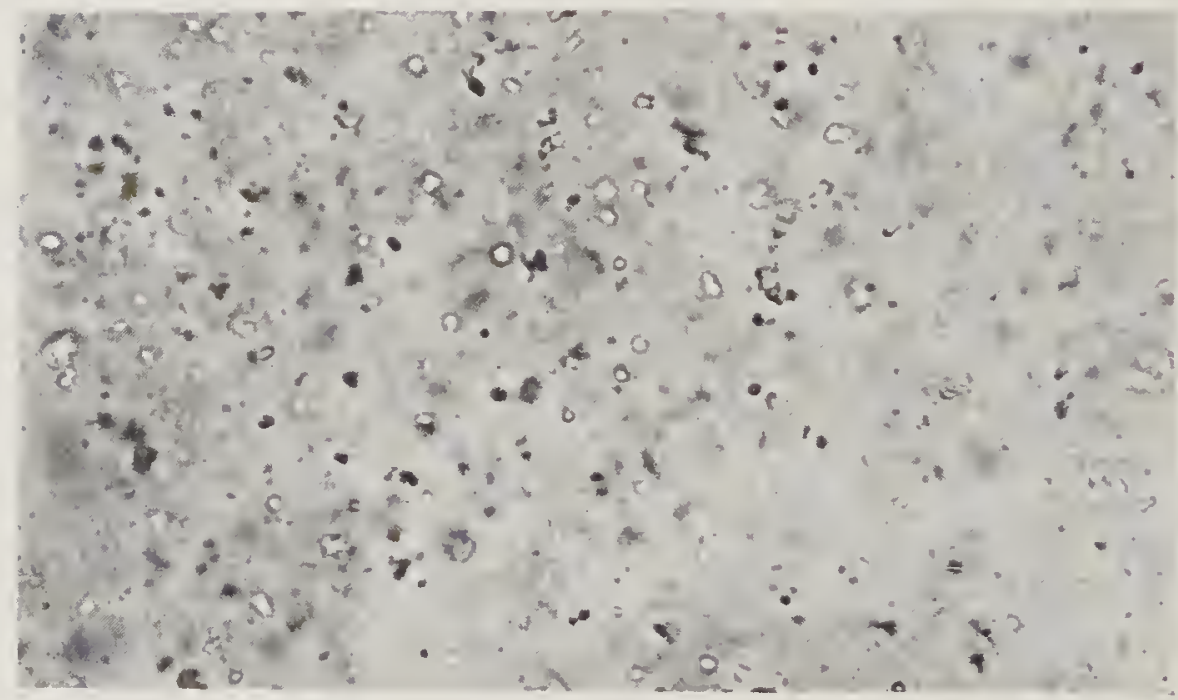

FIG. $37(\times 500)$

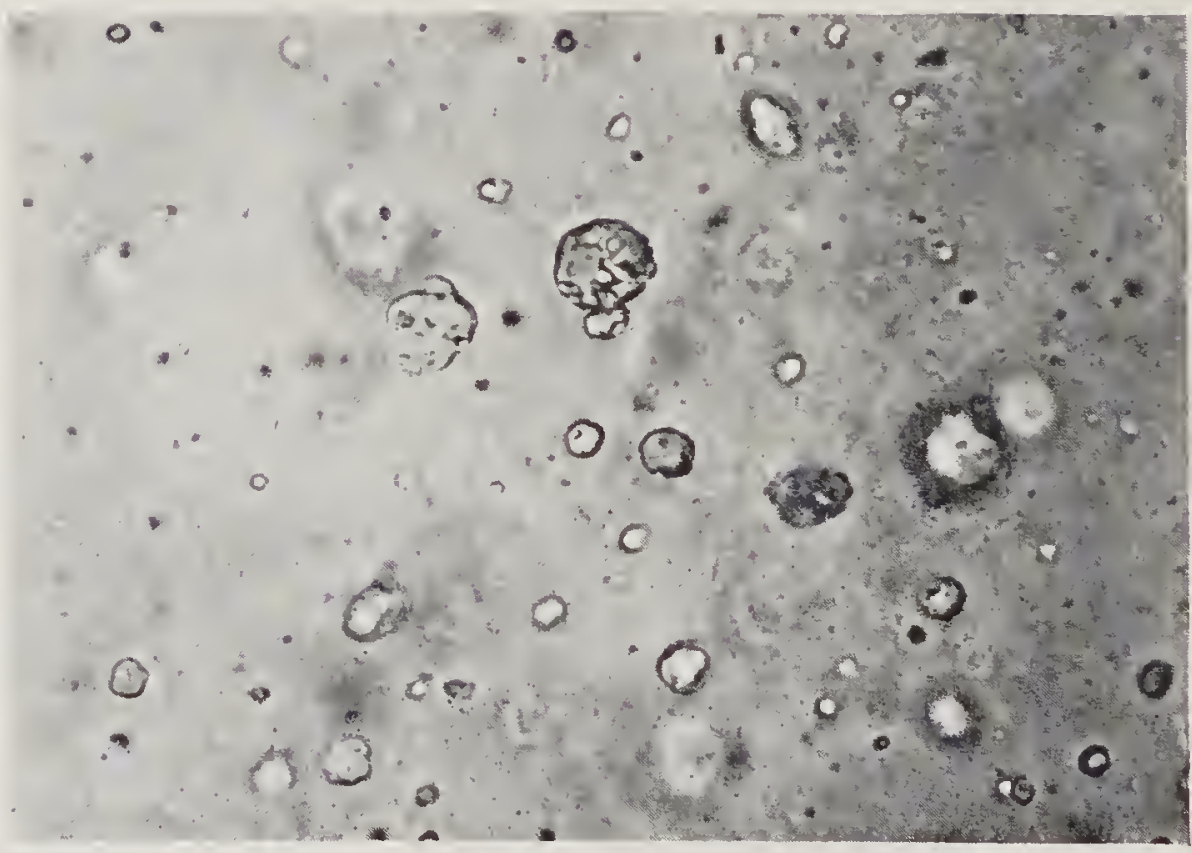

Fic. $38(x 500)$

SHOWING SOME EFHECTS OF HIGH TEMPERATURFS ON THE SAIINE, MEUIA THEMSFLVES 
In Plate XII., Fig. 36 , a portion of a flake is shown that had been heated a few weeks before only to $100^{\circ} \mathrm{C}$., which was crowded with Bacteria, together with only a comparatively small number of inorganic particles. The specks seen in the photograph are principally organic units; in other parts of the flake they were associated with Torulæ.

In Fig. 37 a portion of a flake from an A solution which had been heated to $\mathrm{I} 30^{\circ} \mathrm{C}$. for ten minutes, and subsequently exposed to light (during rather cold weather) for five weeks, is shown. All the flakes were absolutely crowded with large and small inorganic concretions, and no living organisms of any kind were found.

In Fig. 38 a portion of a flake is seen from a tube that had been charged with some of the same solution, and had subsequently been heated to $125^{\circ} \mathrm{C}$. only, for ten minutes. The tube had been exposed to light side by side with the last, and also for a period of five weeks. The concretions in the flakes were extraordinarily large and numerous, and again no organisms could be found. Apart from the temperature to which this tube had been exposed having been rather lower, it differed from the last only in the fact that the tube was composed of uviol glass. The change in the flakes of silica is, therefore, to be regarded as the combined effect of the high temperature, together with the subsequent action of light through this glass. ${ }^{1}$

1 The researches of Prof. Quincke seem to indicate another possible cause of difference of a recondite character. Thus, in a recent paper in The Proceedings of the Royal Socicty (No. 521, A, p. 67) he says:- 
I am disposed to think that the A solution bears high temperatures worse than the AA solution. I have heated very few of the former to $130^{\circ} \mathrm{C}$., but many specimens of the latter, and in none of them have I found a great crowd of large inorganic particles such as are shown in Fig. 37. In some of the solutions, indeed, the flakes of silica have scarcely shown any such particles-many of them in these cases presenting only a uniformly fine granular texture, such as may be dimly seen in Figs. 25 and 28.

It seems clear, therefore, that just as solutions of organic matter are degraded by high temperatures, so are saline solutions liable to be altered in very different ways; till at last there comes a limit to the possibility of obtaining results which appear freely enough at lower temperatures. Certain it is, that in none of these saline solutions containing silica have I, as yet, been able to find living organisms in tubes that had been heated to temperatures above $130^{\circ} \mathrm{C}$. It may be, of course, that longer periods of exposure to light might lead to different results.

I have, however, had some remarkable results with sea-water heated to $\mathrm{I} 35^{\circ} \mathrm{C}$., and even to "A liquid does not possess constant and unchangeable properties at the same temperature and pressure. All liquids in nature form allotropic modifications, such as have been known in the case of sulphur, phosphorus, iron, etc. Such an allotropic modification is formed in larger quantity, the more quickly the fuid is cooled." Some of my tubes after heating, when taken from the bath, have been left to cool slowly, though others have been purposely cooled more quickly, before cleaning and labelling them. No record, however, was kept of such slight differences in treatment. 
I $50^{\circ}$ C., in which Bacteria, Micrococci, Streptococci, and large refractive Torulæ have been found, and when mounted and surrounded with paraffin, with all the care previously indicated (p. 246), all these organisms have multiplied beneath the cover glasses. Moreover, large numbers of Staphylococci have similarly multiplied beneath the cover glasses on two occasions-though never during the whole year have I once seen these latter organisms under any other conditions.

Still, on other occasions, tubes containing seawater that have been heated to such temperatures have remained barren. My experiments with this fluid have been limited in number and not sufficient to enable me to account for the very different results obtained on different occasions. I determined, therefore, not to reproduce the photographs of organisms obtained from sea-water, and to limit myself in this work to an account of the remarkable results obtained with solutions containing sodium silicate as a principal ingredient.

Before making some final statements concerning the major problem with which we have hitherto been concerned, a subordinate though highly interesting question in reference to these experiments, in which silicon has been present and carbon has been ostensibly more or less completely absent, must again be referred to.

The question is whether we are to consider that silicon, whose chemical properties are so very closely allied to carbon, and, like carbon, first appears in 
some of the hottest stars, can take its place, wholly or in part, as one of the constituents of protoplasm. ${ }^{1}$

I do not pretend that my experiments afford any distinct proof that such a substitution is possiblesince carbon may have been present in the solutions, in the way of some accidental impurity, either in the distilled water or in the chemicals employed.

Still, the experiments recorded in this and in the last chapter are very suggestive that such a substitution may be possible, when we bear in mind (a) the freedom with which Moulds will grow in and on the surface of colloidal silica, as originally observed by Roberts and Slack, and subsequently by myself; and when (b) we recognise that minute Moulds and other Micro-organisms will appear within closed tubes containing the solutions in question, though these have previously been exposed to high temperatures. It may be said that in such cases the organisms, if from no other source, have been capable of taking their carbon from the $\mathrm{CO}_{2}$ of the air within the tube; but against this supposition, in the case of the sealed tubes, there is the fact (c) that the organisms are invariably found away from the air, in or upon the flakes of silica which collect at the bottom of the tube; and that for month after month they are to be found there only,

1 According to Ostwald " Inorganic Chemistry," translation, I904, p. 425), "The largest part of the earth's surface is composed of silicon dioxide, or of its compounds : over a quarter of the solid crust of the earth is formed by silicon." And in regard to the silicates of the alkali metals, which go by the name of water glass, he says (p. 427): "These salts are readily obtained by fusing quartz with the hydroxides or carbonates of the alkali metals." 
and never free, in the strata of water intervening between the flakes and the air. There is the further fact $(d)$ that the organisms appear almost, if not quite, as freely in tubes from which the air has been expelled by boiling as in those containing air.

Taken together, these facts seem to me to make out a very strong presumptive case in favour of the view that silicon is capable, to some extent, of taking the place of carbon in protoplasm. ${ }^{1}$

\section{Interpretation of the Experimental Results.}

In regard to the cardinal fact of the appearance of organisms in the experiments that have just been

1 Recently I had the pleasure of showing my photographs to, and discussing this question with, Sir William Ramsay, when he kindly gave me the following statement concerning "bodies analogous to hydrocarbons," in which silicon occurs, and to another compound in which silicon in part replaces carbon:--

" $\mathrm{CH}_{4}$, Marsh gas. $\mathrm{C}_{2} \mathrm{H}_{6}$, Ethane.

$\mathrm{SiH}_{4}$, Siliciuretted hydrogen.

$\mathrm{Si}_{2} \mathrm{H}_{6}$, Silico-ethane.

"The hydrocarbons run up to $\mathrm{C}_{30} \mathrm{H}_{62}$, though no higher compound of silica is known than $\mathrm{Si}_{2} \mathrm{H}_{6}$. But there are compounds in which silicon in part replaces carbon : e.g. Silico-nonane.

$$
\begin{aligned}
& \text { Nonane }=\mathrm{C}_{9} \mathrm{H}_{20} . \\
& \text { Silico-nonane }=\mathrm{SiC}_{8} \mathrm{H}_{20}, "
\end{aligned}
$$

Then, again, Leucone, $\mathrm{SiH}_{4} \mathrm{O}_{5}$, and other allied compounds of silicon, hydrogen and oxygen are referred to by Wöhler (Ann. der Chim. und Pharm., cxxvii. p. 457); while Friedel and Ladenburg have shown that there is a silicic chloroform, $\mathrm{SiCl}_{3} \mathrm{H}$ (ordinary chloroform being $\left.\mathrm{CCl}_{3} \mathrm{H}\right)$, and also a tribasic silicic ether, $\mathrm{SiH}\left(\mathrm{C}^{2} \mathrm{H}^{5} \mathrm{O}\right)_{3}$, as well as other allied bodies in which silicon exists as one atom of each compound. They succeeded likewise in producing an oxychloride of silicon, and other more complex members of the same series (Comptes Rendus, t. lxvi. pp. 539 and 816). 
recorded, this seems only capable of receiving one interpretation. All the old objections to the notion of their de novo origin have again been fairly met. The organisms found have undoubtedly been living, and they have occurred in tubes which had been Alambés, before they and their contents were submitted to lethal temperatures.

It is now generally admitted, as we have seen, that all micro-organisms, in their full-grown condition, are killed by the briefest exposure in fluids to $100^{\circ} \mathrm{C}$. - to say nothing of lower temperatures.

The spores of Bacilli are recognised as the only products of micro-organisms capable of resisting such an exposure. Yet, as we have found, various other forms that are admitted to be killed at $\mathrm{s}_{0}{ }^{\circ} \mathrm{C}$. can, and do, constantly appear within closed vessels which have been heated to this temperature for ten to twenty minutes. This applies, among other less known forms, to Bacteria, Vibriones, Micrococci, Streptococci, Torula, and other germs of Moulds. When these organisms appear under such conditions, therefore, we can only conclude that they must have been evolved de novo.

In regard to ordinary Bacilli, the position, as we have seen, is different. These particular forms possess "spores," which, at all events after they have undergone desiccation, are capable of resisting a higher degree of heat. The extent of their powers of resistance has been differently estimated, but after prolonged researches in the Pasteur Institute, the point was formerly fixed by the master himself at I $10^{\circ} \mathrm{C}$., and later, after two years' work, could only 
be raised by his assistant to I I $5^{\circ} \mathrm{C}$. At this point, it is proclaimed, all the spores of ordinary Bacilli are completely and rapidly killed. Noting by the way that even this temperature is much higher than Bacilli spores were shown to be capable of withstanding in my direct experiments (see pp. 71-86), and, further, that such bodies are little likely to be found in the freshly distilled water, or in either of the chemicals made use of in my experiments; let us, for the present, accept this temperature of I I $5^{\circ} \mathrm{C}$. as needful to destroy all the products of ordinary Bacilli.

But, as we have seen, such bodies, as well as Vibriones, Cocci, Streptococci, Torulæ, and other germs of Fungi, have appeared within our experimental vessels when they have been heated for from ten to twenty minutes to temperatures ranging from I $5^{\circ}$ to $\mathrm{I} 30^{\circ} \mathrm{C}$. These organisms which we have seen to be living-which developed and multiplied-must, therefore, have been evolved de novo. What other answer is it possible to give?

All the bacteriologists throughout the civilised world during the innumerable researches they have carried on for the last five and thirty years have only been able to find certain so-called "Thermophilic Bacilli" in the soil that are capable of resisting higher degrees of heat than I I $5^{\circ} \mathrm{C}$. Yet these low organisms, which are said never to be found even in tap-waters, and a fortiori not in recently distilled waters or in either of our chemicals, have, according to Christen (see p. 85), never shown signs of life after they have been exposed to 
"I $25^{\circ}$ to $130^{\circ} \mathrm{C}$. for five minutes and longer; to I $35^{\circ} \mathrm{C}$. for one to five minutes; or to $140^{\circ} \mathrm{C}$. for one minute."

The moist resistant forms of these spores of the soil Bacilli, when examined by W. H. Park, succumbed, however, at temperatures lower than this. He says they were destroyed "by exposure for twenty-five minutes in steam at $113^{\circ}$ to $116^{\circ} \mathrm{C}$, and in two minutes at $127^{\circ} \mathrm{C}$." 1

It comes to this, then, that all the organisms found in my experiments, with the exception of Bacilli, are such as would be killed at $100^{\circ} \mathrm{C}$; that these latter, so far as they could by any possibility be found within my tubes, should have been killed at $115^{\circ} \mathrm{C}$., yet Bacilli, as well as Bacteria, Vibriones, Micrococci, Streptococci, Torulæ, and other Fungus-germs, dying under $100^{\circ} \mathrm{C}$., have been taken in large numbers from tubes that had been heated to $115^{\circ}$ I $30^{\circ} \mathrm{C}$., for from ten to twenty minutes.

Elsewhere, I have said," "we have in reality two distinct and more or less independent methods for attacking the problem as to the present occurrence of Archebiosis--that $(a)$ by experiment with superheated fluids in closed flasks; and $(b)$ another less recognised method, that of mere observation, aided by high microscopic powers, of what occurs in thin films of suitable unheated organic fluids. This latter method calls attention to, and thoroughly exposes the fallacy of, the common belief that the occurrence

1 "Pathogenic Micro-organisms," 2nd Edition, I906, p. 45.

$\because$ "The Nature and Origin of Living Matter," r $905, \mathrm{p}$. I 5 S. 
of 'spontaneous generation,' is opposed to the universal experience of mankind. It shows that what is supposed to contradict this common experience lies altogether outside, and, of necessity, completely beyond the range of ordinary human experience. Nobody with unaided eyes could ever have witnessed the birth from fluids of invisible particles, by which 'spontaneous generation' of living matter must always commence, if it commences at all. And, even when aided by the most powerful microscope, nobody could decide when the minimum visible particles appear in the field of view, that such particles have proceeded from invisible germs of preexisting organisms, rather than from a primordial synthesis of living units. But in regard to this latter point, I have already shown that absolutely no logical or consistent reason exists for a disbelief in the present occurrence of Archebiosis." There is, it was stated, "no vestige of evidence to show that under favourable conditions the process may not be continually taking place all around us."

But what is assumed to be continually taking place in free nature-in ponds, lakes, rivers, the sea, and in innumerable other sites-must be regarded as a process far more easily brought about than can ever be possible under the very restrictive conditions which can alone exist in our experimental vessels, where we have to do with small quantities of fluid, more or less degraded by a preliminary heating, and enclosed within small glass vessels. Yet, as I have endeavoured to show in this work, by all the evidence recorded in Chapters $\mathrm{x}$.-xii. concerning 
the thermal death-point of living organisms and their germs, together with my new experiments with saline solutions, the evolution of living things is capable of occurring even under such unfavourable conditions.

We are bound to conclude, in fact, that there must be in nature a distinct proclivity to the formation of living matter. We are almost driven to such a conclusion when we see the freedom with which it can be produced in a simple inoculated solution of ammonium tartrate in distilled water (see p. 23.5). There we have the growth and multiplication of very varied micro-organisms occurring under conditions so simple as to be almost incredible. Now, again, we are confronted with facts hard to be believed and difficult to understand-yet incontestable-when we find varied forms of Bacteria and Torulæ appearing plentifully in hermetically sealed tubes which have been submitted to a scathing degree of heat-far more than is sufficient to destroy all other known forms of like kind. They are not there at first after the tube has been heated; though after a time they are to be found in abundance. But they are invariably motionless, and must, therefore, have developed in the sites where they are found.

The wonder too is enormously increased when we think of the simplicity of the media in which this life-giving process occurs. The simplicity of the conditions, in fact, for the "origination" of life in my latest experiments, is only equalled by the simplicity of the conditions sometimes sufficient for 
the "growth and multiplication" of already existing living things - as shown by their rapid increase in the simple ammonium tartrate solution in distilled water: in which at least seven different kinds of micro-organisms were found growing side by side. ${ }^{1}$

\section{Other Confirmatory Evidence}

A related de novo production of similar organisms by Heterogenesis I have elsewhere proved to occur. That is, just as in the superheated tubes, where living Bacteriæ were previously non-existent, such units can be made to appear; so they can be made to appear within the substance of pre-existing living things that were previously germless : and there also they develop from particles so minute as to be previously invisible. Reference may be made to two such cases-one occurring within the tissues of a vegetable, and the other within those of an animal.

It was strongly held by Pasteur that the cells of healthy plants are germless, and this view is still generally regarded as true. Bacteriologists cannot obtain evidence of the existence of micro-organisms in cells taken from the centre of a healthy potato or turnip, and yet Bacteria can be caused to appear in these situations, although adequate precautions are taken against infection. The method I have adopted is as follows :-

A small new potato is first well washed in water, then it is put into a screw-top bottle containing a ro per cent. solution of formalin, and allowed

${ }^{1}$ See Knowledge anc Scientific Nerus, Aug. 1905, p. 200. 
to soak therein for twenty minutes, the fluid being occasionally shaken, so as to cover the whole internal surface of the bottle. The solution is next poured out, leaving the surface of the potato and the bottle wet with the antiseptic fluid, and the cover is tightly screwed on. When this method was employed there was never the slightest indication of any contamination of the surface of the potato: yet after it had been left for a number of weeks in this closed and aseptic bottle micro-organisms were often found (after careful search) within multitudes of closed cells - that is when sections were taken from central regions of the potato, but not from peripheral portions which had been acted upon by the antiseptic fluid. Representatives of such organisms as well as of others obtained from the cells of a turnip, preserved in such a way as to be absolutely free from all chances of external contamination, are to be seen in my work "Studies in Heterogenesis," Plate XVII., Figs. 193-I97, and also in "The Nature and Origin of Living Matter," Plate II., Figs. 4, 5, 6.

In all such cases the micro-organisms seem first to show themselves as motionless specks in the primordial utricle of the closed cells, and the specks develop into such Bacilli as are shown in the figures. The fact that the first things to appear within the closed cells are mere motionless germs suffices of itself to negative the possibility of infection. Mere motionless germs could not infect. Vegetable cells can only be penetrated by adult organisms capable of secreting toxins for killing the cells, and a cytase for softening their walls. 
Infecting Bacteria must be endowed also with mobility in order to be enabled to penetrate the cell wall which they have previously softened.

The only other possibility, apart from heterogenesis, of accounting for the appearance of the Bacteria within the closed cells would be the supposition that germs of micro-organisms have been "latent" within the cells. But of this no proof has ever been given. Bacteriologists fail to find evidence of their existence, and the verdict of science has, in fact, been adverse to this supposition.

Let us now take another case and one in which the development of Bacteria from ultra-microscopic particles can be watched stage by stage. In Cyclops, a little water-flea, the abdominal appendages are furnished with a number of spines or setæ, in the interior of which only structureless protoplasm is to be seen, free from granules. This protoplasm is so clear that it has a glass-like appearance. When one of these little creatures is placed in a drop of distilled water on a microscope slip, and a covering glass is applied, it soon dies. We may then examine some one of the larger spines from day to day. At the end of a couple of days, when the specimen is kept in a damp chamber and the weather is warm, instead of protoplasm free from all visible particles, such as originally existed, there is the first appearance of some minute motionless specks, which gradually increase in number and in size till some of them show themselves as motionless Bacilli. About the sixth day, some of these Bacilli begin to exhibit the first signs of movement, and soon we have a seeth- 
ing mass of such organisms in the substance of the still intact spine.

How, again, are we to explain these appearances? The spine has a chitinous envelope which could not easily be penetrated by active full-grown organisms, much less by motionless germs, even if these existed in the distilled water. Thus infection from without is here again scarcely to be thought of. We see motionless specks appear and slowly develop into motionless Bacilli. To assume the pre-existence in the protoplasm of the spine of multitudes of ultramicroscopic germs, for no other purpose than to stave off a belief in the de novo origin of Bacteria, is surely neither scientific nor permissible. And yet my critics hint only at the existence of "latent germs," without attempting to offer any evidence of their existence, or of any previous belief in this direction. Truth-seekers are thus left with a simple choice: either $(a)$ a belief in the existence of latent germs independently of all evidence; or $(b)$ a belief in the de novo origin of Bacteria on very cogent evidence.

In my work, "The Nature and Origin of Living Matter," Chapter ix., other instances of this de novo origin of Bacteria by heterogenesis are cited, and in all such cases what can be seen is this-particles becoming visible in the midst of more or less homogeneous protoplasm, such particles being invariably motionless, but followed soon by their development into definite Bacteria or their allies, recognisable as such by their shapes and modes of collocation. There is, therefore, in all such cases an appearance altogether different from that of adult organisms in 
a state of activity, such as would be seen if we had really to do with a case of infection.

The similarity in the mode of origin of the Bacteria and Torulæ through the flakes of silica in my recent experiments, to that which occurs in these processes of heterogenesis is therefore very striking-we have always the minute motionless specks, gradually becoming visible and taking on characteristic shapes, just as crystals do as they separate from their parent media, and just as the doctrine of evolution would seem to require. In reference to this fact of the new-born units developing into well-known forms - which some find so difficult to understand-the following remarks, made elsewhere, may be quoted :- 1

"There would be, in fact, just as much reason why the newborn organism should develop into the form of one already in existence, as there would be that the crystal of sulphate of soda which forms to-day in a solution of that substance should resemble that which formed under similar conditions twelve months or a hundred years previously. He who believes in the uniformity of natural phenomena could anticipate no other result. Living matter, which we believe to be now produced de novo, speedily shapes itself into some well-known form; and so also new crystalline matter, which may have been produced synthetically by the chemist in his laboratory, falls habitually into one or other of the known crystalline systems."

"It seems, therefore, no more wonderful that the simple Mould that develops de novo to-day should resemble another which develops from the spore of a pre-existing organism, than that a crystal forming independently to-day in a saline solution should resemble another which is capable of arising by the growth of a fragment detached from a similar pre-existing crystal. In all these

1 "The Nature and Origin of Living Matter," 1905, p. 287. 
cases there is a similarity of product, because the crystalline or organic form produced is to be regarded as the physical expression of the harmonious actions which have led to its productionbecause the forms are the results of a physical necessity, and not of a mere blind chance."

Recent observations have indeed shown that the absolute constancy in regard to the form of crystals is far more strict than was previously supposed. Thus A. E. H. Tutton in an interesting article on "The Study of Crystals" says: ${ }^{1}$ "One of the main results of recent investigation has been to prove that the beautiful exterior shapes of crystals, as defined by the geometrical angles between the numerous truly plane natural facets, rather than by the relative development of those planes, is an invariable and characteristic property of the substance composing the crystal, by which that substance can be identified from all the countless other substances which are known to exist." Moreover, he adds, "the refractive power and the behaviour of the crystal in polarised light afford immediate evidence of identity almost as valuable as the angles between the natural facets."

The constancy of these properties in crystals in accordance with their molecular composition and structure is an interesting fact, when we consider that crystals are always born anew-that heredity has nothing whatever to do with their properties. Similarly, I would say that the Bacteria and Torulæ that are ever being born anew can owe nothing to heredity, but that their shapes and properties must

1 The Times, Engineering Supplement, Sep. 19, 1906, p. 298 
be solely due to their ultimate molecular composition.

And when we think of the marvellous molecular complexity of protoplasm, and the innumerable variations of isomeric type that would be possible in new-born living units, we may see our way, in a measure, to understand the existence of such countless transitional variations in form and in property as are to be found among Micrococci, Streptococci, Staphylococci, Bacteria, Bacilli, Vibriones Spirillæ, etc.-and such as bacteriologists describe in their text-books.

My point of view is that these multitudes of varied forms and properties may be found in new-born living units, altogether apart from heredity ; just as the multitudes of new-born crystals, when they appear, have their own exact angles between their facets, and their own optical characters, whenever they are formed under similar conditions and at whatever intervals of time. There is one important difference, it is true, between the two kinds of units. The simpler molecular composition of the crystal, which is a statical aggregate, does not predispose to change; while the infinitely more complex molecular architecture of the living unit favours change in form and in property under varying external conditions, as bacteriologists have long ago found--though without such change in conditions they continue to "breed true."

I have, however, elsewhere endeavoured to show, not only, that Bacteria of different kinds, Torulæ and 
Moulds often originate de novo by heterogenesis, but that "various simple Algæ, Diatoms, and Phytozoa may take their origin from alien sources; and that a similar heterogenetic origin is most frequently met with for Amœbæ, Actinophrys, Flagellate Monads, Peranemata, and even Ciliated Infusoria. ${ }^{1}$ "

These views of mine, as the results of oft-repeated observations, are, I would submit, in accordance with the present-day existonce of vast multitudes of lowest organisms of all kinds on the face of the earth. No consistent explanation of their presence can be given by those who will not accept my facts. They have been frequently challenged to do so, but they never attempt to furnish an answer to the following considerations (loc. cit. p. 299) :-

"If all the forms of life that have ever existed upon the surface of the Earth have been derived from the primordial forms which took origin by natural synthetic processes occurring only in an incalculably remote past, no adequate and consistent explanation would be forthcoming of the undoubted existence at the present day of the teeming multitudes of such lower organisms as have been above referred to. For if the assumed gradual development of higher forms of life, during past geologic ages, has been largely due to the intrinsic mutability of living matter, as the Evolution hypothesis assumes, would it not be a stultification of that hypothesis to suppose that such primordial forms as Bacteria, Torulæ, Monads, Amœbæ, and Ciliated Infusoria have remained practically unchanged, and in these

"The Nature and Origin of Living Matter," p. 299. 
low grades, for untold millions of years ? Yet those whose views are at present most widely accepted would have us believe that ages and ages before the advent of Man or his immediate predecessors upon the earth, the ancestry of the Bacterium, the Amœba, the Monad, the Mould, or any of the low infusorial animalcules now to be seen in their respective habitats, had been tenants of our globe. The mere suggestion seems to carry absurdity on its face. If this were really so, then we could only expect that such forms would be the very types of conservatism and stability; whereas, as a matter of fact, all such organisms are rather the best types of change and mutability.... It is impossible to say that they have preserved their primitive forms by reason of their existence in unchanging environments. The very reverse of this must have been the fact; and even now such organisms are to be met with abundantly over the face of the earth, and in the most diverse situations. . . . But in accordance with the views advanced in this work, the present-day existence of these organisms may be fully explained, and is just what might be expectedif they are ever seething up anew by Archebiosis and Heterogenesis." 



\section{PART VI}

THE RELATION OF MY WORK ANI) VIEWS TO

MODERN BACTERIOLOGY

\section{CHAPTER XXI}

THE RELATION OF MY WORK AND VIEWS TO MODERN BACTERIOLOGY

SINCE this, in all probability, is to be my last $S$ contribution to the subject discussed in the present volume, and as many misconceptions exist in reference to the relations of my work and views to modern bacteriology, it seems fitting that I should say something here on this subject; and should, moreover, explain why during a long series of years I have devoted so much of my leisure time in attempting to throw light on this question of the Origin of Life-why during recent years I have, in fact, again taken the subject up with renewed vigour, though knowing well that, for the present, I should meet with little other than unsympathetic and hostile criticism.

My interest in the question has all along been twofold-partly from the point of view of the biologist, and partly from that of the pathologist and physician. 
The fascination of the subject from the biological standpoint has in a general way been indicated in this volume, though it has been more fully considered in my work on "The Nature and Origin of Living Matter," where I have attempted in Chapter xiv to show how needful is the recognition of the reality of Archebiosis and Heterogenesis from the point of view of the Doctrine of Evolution; and what light a belief in the occurrence of such processes is capable of shedding upon the past history of our earth, as well as upon the meaning of the existence almost everywhere at the present day of swarms of the lowest forms of life, which ought otherwise (that is, apart from the processes in question) to have wholly disappeared ages and ages ago.

Let us look here, however, briefly to the question why, from the point of view of the physician and the pathologist, my interest was originally roused to consider this problem, and why my original point of view still impresses me as strongly as ever, notwithstanding all the remarkable advances that have been made in bacteriological science--which many, wrongly enough, consider to be adverse to my views. In reality, as I shall show, there is no necessary antagonism between my facts and views and modern bacteriological discoveries.

But how needful it is for me to explain my position may be gathered from the fact that the reviewer of my "Studies in Heterogenesis," in one of the leadingmedical journals, said that the book was "the best possible statement of the case against the claims of 
modern bacteriology." This was the dictum of the reviewer, his à priori point of view, though in that volume I had said not one single word tending to minimise the enormous value of bacteriological work. Again, in another important journal the reviewer said, "If, on the other hand, Dr Bastian be correct and the microbes of definite diseases can arise de novo either from harmless organisms ${ }^{1}$ or from unorganised matter, then practically all current ideas on the modes of dealing with epidemic diseases must be abandoned, and an unhappy world must bow before the malevolent caprices of nature." This, as I shall now attempt to show, is untrue in fact, and absolutely contrary to the implications naturally following from my facts and doctrines.

Bacteriologists, almost if not quite universally, regard the low organisms with which they are concerned as capable of arising only from others of like kind, and since it has been shown that there is a constant relation between many of these organisms and definite diseases-that the diseases are, in fact, often actually caused by them-the notion has gradually become strengthened that such diseases, which possess the property of being contagious, can no more arise de novo than the organisms by which they are caused. Thus ultra-contagionist views are regnant, and the frequent de novo origin of infectious diseases, in which I have always believed, is denied.

A late distinguished president of the Royal College of Physicians, whom we all revere, a short time since gave his sanction and adherence to the popular

\footnotetext{
1 No italics in original.
} 
view when he said ${ }^{1}$ : "If I can trace contagion in a very large number of the so-called specific diseases, I consider it more reasonable to assume contagion in the minority than look about for another cause." And he went on to say that, as many of these diseases are associated with the growth and multiplication "of living specific organisms" a belief in the de novo origin of these contagious diseases would "imply also a belief in spontaneous generation." This latter view, although it is so prevalent, is surprisingly fallacious.

There can be no doubt, of course, that the establishment of the reality of "spontaneous generation" must certainly have a very potent influence in gradually breaking down the notion that communicable diseases, simply because they are associated with "specific" micro-organisms, cannot arise de novo. If such organisms can arise de novo, of course the diseases could arise de novo; and, further, the fact of such an origin for microbes would easily carry with it the certainty that they would be extremely mutable under the influence of changing conditions, though otherwise capable of "breeding true."

Still, independently of a belief in the spontaneous generation of microbes, there is another and simpler way by which the "specific" micro-organisms may enter upon the scene. This mode seems to have been lost sight of by the distinguished authority to whom I have last referred, while it was mentioned only to be repudiated by the second reviewer above quoted. Yet bacteriologists themselves have gradu-

1 The Lancet, Oct. 3I, 1903. 
ally found that microbes are extremely mutable, and that the barrier, formerly supposed by many to be impassable, between what are known as non-pathogenic and pathogenic organisms respectively, has been gradually broken down. The development of their science has been steadily giving rise to a great change of view in this respect, as it is now quite easy to show. But if the common "harmless organisms" referred to in the Saturday Review article, could in any way be gradually transformed so as to take on new properties identical with those possessed by the organisms associated with this or that contagious disease, a way would be found for the de novo origin of such diseases, though there would only be transformation of organisms, by whose aid, when thus modified, these same diseases might be spread indefinitely from person to person.

That these are not my views only, but those of bacteriologists high in authority may be easily shown. It will be easy also to adduce instances in which such processes are believed to occur.

Prof. Hueter said long ago," "Although it is impossible not to recognise the specific modes of activity of micro-organisms in the production of infective diseases, we need not on that account deny that there is a certain unity in all these micro-organisms. I am of opinion that this unity is founded upon the processes of putrefaction, and that the specific modes of activity must be regarded

1 "Transactions of International Med. Congress," I88I, vol. i. p. 329 . 
as depending upon certain alterations in the putrefactive process." Prof. Hueppe of Prague has also quite recently expressed the very similar view that the origin of all common infectious diseases is "phylogenetically traceable to putrefactive processes." 1

If we turn again to one of the best modern books on this subject, the "Principles of Bacteriology," by Lehmann and Neumann, we find the following important statements (I90I, pp. I I 8-I I9), "The division of bacteria into pathogenic and non-pathogenic, etc., as is still always done in text-books, has failed absolutely. We can understand and know the pathogenic varieties only if we study simultaneously the non-pathogenic, from which the former have once originated and still always originate." They say also, "We certainly believe it belongs to the future to convert varieties of bacteria into others in a manner scarcely to be imagined to-day. The forms of the Micrococcus pyogenes are convertible into each other; the Bacterium pyocaneum and Bacterium fluorescens can indeed almost certainly be converted into each other; and similar statements regarding typhus and coli, diphtheria and pseudo-diphtheria, etc., are always still looked upon with scepticism, but the possibility, nay even the probability, can scarcely be contested any more."

Let me now cite a few illustrations of these

${ }^{1}$ Harben Lecture, in The Journal of State Medicine, Nov. 1903, p. 64 I. 
doctrines in connection with some important infectious diseases.

Elsewhere I have given an account of very conclusive evidence showing that some of the forms of Septicæmia have been experimentally demonstrated to arise from common micro-organisms, and yet, when thus established, they are found to be associated with so-called "specific" organisms, capable of acting as contagia for the spread of these diseases to other animals. What actually occurred, and its significance, was thus referred to : ${ }^{1}-$

"The production of two different forms of septicæmia by the inoculation of some of the same putrid material into different sites is a matter of the greatest importance. The putrid blood under the skin gives rise to one form of specific microorganism and contagious disease; while two or three drops of the same putrid blood introduced into the peritoneal cavity of a similar animal give rise to swarms of a different organism and the development of another contagious affection. The differences in the inflammatory processes in the two situations are capable, that is, of transforming some common micro-organisms into two quite different specific Bacilli." Here, then, we have a striking illustration of the views originally expressed by Professor Heuter and more recently by Hueppe.

There is reason to believe, again, that the so-called typhoid Bacillus of Eberth is only a modification of an extremely common putrefactive organism. Thus

1 "The Nature and Origin of Living Matter," 1905, pp. 316-320. 
Rodet and Roux say," "The organisms described under the names bacillus coli communis and the bacillus of Eberth belong to the same pathogenic species, of which they form two varieties removed one from the other when they present their classical characters, but related the one to the other by a series of intermediate forms." Dr McWeeney, the bacteriologist of the Local Government Board in Ireland, has also said," "He did not believe that there was an essential distinction between the typhoid Bacillus, and the Bacillus coli communis" which is habitually to be found in the intestine. These bacteriologists agree, therefore, with Lehmann and Neumann, who say such a transformation "can scarcely be contested any more." Under what particular set of conditions the one form passes over into the other is at present unknown-though there is much very strong evidence, as many believe, tending to show that typhoid fever occasionally arises de novo. It occurs, that is, in isolated cases, under conditions in which it is almost impossible to believe that contagion can have been operative.

An epidemic of Diphtheria recently occurred on Dartmoor, in the neighbourhood of Princetown, which was carefully investigated by $\operatorname{Dr}$ Deene Sweeting of the Local Government Board. His report is valuable from the light that it throws on the de novo origin of this contagious diseaseespecially as previous reports concerning other epidemics of the same malady by skilled investi

1 Lyon Médicale, 1891, lxviii. p. 325.

$\because$ Lancet, 1896, i. p. 995. 
gators have testified to much the same mode of origin. No single factor of unwholesomeness at Princetown could be pointed to as having been associated with the observed incidence of the disease; but the clue to an explanation was afforded by a general prevalence of much and marked sorethroat in the district, and by the tender age of the children first attacked. Dr Sweeting says :-

"There seems, therefore, reason to suspect that diphtheria was evolved amongst young children from previous minor sore-throat in the village, and developed itself in the infants' class at the school; that the disease thus set up was kept going by assemblage of susceptible children at school, and by the further reinforcement of sore-throat when the school was closed; and [the disease] was spread by infection from person to person at school and elsewhere."

Further, in a recent able communication by Dr Hubert Biss on the "Borderlands of Diphtheria and Scarlet Fever," written after a large experience in a fever hospital, we find him saying," "The nuances between these conditions-scarlet fever, diphtheria, and tonsillitis-are so gentle that each shades off into the other not at one but at many points." And quite lately he has reiterated his view that the two diseases, scarlet fever and diphtheria, are to some extent interchangeable. He says, "But there was no question of theory. The metamorphosis actually took place. Scarlet

1 The Lancet, 1903, ii. p. 1296.

2 Prit. Med. Joum., I906, ii. p. 895. 
fever patients infected their friends with diphtheria, and diphtheria patients in their turn infected their friends with scarlet fever." $\mathrm{He}$ is so positive as to what he has observed, that he speaks of the transformation as "an established fact."

Of course, if this is true in regard to the generation of diphtheria from common sore-throats under certain conditions; and if a diphtheria patient may at times give rise to contagia which set up scarlet fever in another, we have practically a de novo origin for this latter disease also ${ }^{1}$ and in each case it can only be supposed that minute transitions in the activities or properties of the micro-organisms concerned must have occurred; so that so-called common or non-pathogenic organisms in the throat have been converted into pathogenic forms, and that, among these, under special conditions, other variations have been brought about, leading to an apparent transformation of one disease into another.

Lehmann and Neumann again, in their "Principles of Bacteriology," call special attention to the probable mode of origin of that fearful scourge Pest, more commonly called Plague. They cite it as affording an important example of that transformation of non-pathogenic into pathogenic organisms, which we are now considering, and in which they also are firm believers. They say (p. 2I7): "In India, Hankin and Yersin have many times culti-

1 From time to time cases of Scarlet Fever have been reported in which it is almost impossible to believe that they have not been engendered de novo. An extremely well-authenticated case of this kind has recently been recorded by Dr R. J. Mackeown of H.M.S. Alacrity (see Brit. Med. Journ., June I I, 1904, p. 1370). 
vated non-virulent varieties of bacilli, resembling very much the pest bacillus, from the environs of men in houses infected with pest. . . . Pest occurs spontaneously in rats. Epidemics of pest in rats often precede those in man. It appears as if certain tropical soil bacilli first become acclimated to the rat's body, and then are transferred to man." 1

In ways thus indicated, then, quite independently of any de novo origin of micro-organisms, we may have such contagious diseases as septicæmia, typhoid fever, diphtheria, scarlet fever, plague, and other communicable affections arising de novo, in consequence of the transformation under this or that set of local conditions-modifications of "soil " in fact -of some common, ever-present micro-organisms. From common organisms, they become "specific" or pathogenic organisms, and contagious diseases are thus engendered, which may spread more or less widely among the community.

It is astonishing, however, how reluctant pathologists are to admit such possibilities - and how they still cling, in spite of the most obvious teachings, to ultra-contagionist doctrines.

In conclusion, I will give one notable instance of this, touching the origin of a fearfully common malady which not many years since was regarded as far more frequently having a de novo than

1 It is almost universally held by sanitary officers in India, who have been engaged in dealing with the recent epidemic, that the disease in rats is principally responsible for the spread of plague; and, as a corollary to this statement, all the evidence that has been accumulated seems to show that rat-fleas are the chief agents in the transmission of the disease. 
a contagious mode of origin. In a recent address ${ }^{1}$ by a distinguished pathologist, Professor Adami of Montreal, he cites a very distinct case in which certain common Bacteria under the influence of altered conditions of life have, within a comparatively brief period, had their metabolic processes completely altered; so that, as he says, "From having been perfectly harmless they are now pathogenic, and can set up disease." He then makes the following remarks: "What is to be said concerning the tubercle bacillus in this connection? In the first place we may have the complete assurance that Adam was not created suffering from tuberculosis. The bacillus, we may be fairly sure, from living it may be on food-stuffs outside the body, accustomed itself first to living on the surface and in the passages of the organism as a harmless saprophyte, and only later gained the power of living not on, but in, the tissues, and from that moment it became pathogenic." So far so good, but then he goes on to say, this "must have happened centuries and centuries ago." We may wonder on what evidence he comes to such a conclusion; and, from my point of view, we may be astonished to find that he arrives at such a conclusion simply because phthisis was well known to early Greek writers on medicine.

In the light of the illustration that Adami had previously given, and of many other well-known facts of like kind, showing that a few days, or at most a week or two, will generally suffice for the 1 Brit. Med. Journ., 1905, i. p. I1 35. 
conversion of non-pathogenic into pathogenic organisms, we may well be surprised at his thinking that a process which he assumes to have occurred once in the days of the early Greeks, and before their time, may not be taking place now - as it may have done through all intervening periods of time. Like another eminent pathologist, however, he possibly inclines, for some mysterious reason, to the view that "the origin of the germs of disease was probably in the remote geological past."

What has just been said is equally applicable to the views of $\mathrm{Dr}$ Andrewes as expressed in his recent Horace Dobell Lecture (Lancet, Nov. 24) on "The Evolution of the Streptococci." Noting by the way that the bourne of Evolution is surely not parasitism, we find that what he speaks of is merely specialisation. But to enable these primitive organisms to pass from the prototrophic to the metatrophic mode of nutrition he imagines incalculable periods of time to have been needed, and speaks of it as having been brought about through the agency of " natural selection." Though how, in these non-nucleated organisms, natural selection is to act he does not venture to hint. Where, as here, chromosomes are absent heredity, according to current notions, would be impossible, and without heredity no "natural selection" could possibly come into operation.

Of course the views referred to, which are very prevalent, are a kind of reflex of the notion that Archebiosis itself occurred, once only, in the remote past, and has not been repeated. Growing out of such a view there comes the notion that the 
Bacteria of to-day are heirs of all the ages, having descended from an untold number of generations, rather than being often, as I believe, things of yesterday - that is, units continually born anew, with recurring similar forms and properties, just as it happens with different kinds of crystals : the forms and properties in each case being the necessary and invariable results of the molecular composition of the units in question.

But, even from the point of view of such doctrines as are now not uncommonly held by bacteriologists (many of whom are disposed to take such a view concerning the origin of Plague as I have quoted from Lehmann and Neumann), we may well ask why the tubercle Bacillus may not also frequently arise from other common Bacilli, when these gain access to lungs, joints, or glands in persons whose general condition predisposes them to scrofulous or tubercular affections. The change of doctrine concerning phthisis, to which I have previously referred, is altogether astonishing, when one thinks of the extreme paucity of direct evidence that exists in support of the prevalent view of the exclusive spread of this disease by contagion.

It is now the fashion to suppose that the fragmentation of dried sputa from phthisical subjects gives rise to the dissemination of contagious particles, which, taken into the lungs of susceptible persons, set up the disease anew in them. It is well to look, however, more closely into such a view, and to ask what direct evidence there is in favour of this as a common mode of dissemination of the disease. 
With regard to human beings exact and perfectly convincing evidence is almost entirely absent. It is well known that Behring disbelieves in this very commonly accepted view; and that it is beginning to be more generally discredited by others, is shown by the fact that, in a recent number of the British Medical Journal, the very possibility of such a mode of infection is questioned, as may be seen from the following statement (November io, I 906, p. I 323): "It is generally held that the peribronchial nodules frequently observed represent the initial lesion in pulmonary tuberculosis, and that these result from the direct introduction of germs carried in the air. On the other hand, evidence is not wanting that the penetration of air-borne Bacilli into the lungs is difficult, if not impossible."

And, even supposing that air-borne germs, contained in minute fragments of pulverised sputa, are capable of gaining access to the lungs, two other questions arise, to which an answer should be obtained: (1) Is the dried phthisical sputum a substance easily capable of being pulverised? and (2) are germs contained in dried sputa, which have undergone pulverisation, likely to retain their activity and prove efficient contagia?

Some experimental investigations in reference to these latter points have recently been made by Cadéac, ${ }^{1}$ and his results are decidedly adverse to the still fashionable hypothesis as to the transmission of tuberculosis by the inhalation of dust derived from dried sputa. Thus he found (I) from experi-

\footnotetext{
1 Lyon Médicale, December 10, 1905.
} 
ment that the expectorated matter dried slowly, and that its conversion into dust was neither simple nor easy. Spread thickly upon a glass plate and exposed to ordinary daylight in the laboratory, the sputum adhered in drying as a bright varnish, and was not easily powdered into dust till the tenth or twelfth day. While as regards (2) the activity of this dried sputum, he found that even on the sixth day a considerable quantity of the powder was required to cause a discrete peritoneal tuberculosis, as a result of inoculation. Again, the sputum spread upon a marble plate, and kept above a stove for fourteen days, was found to have lost its virulence for the guinea-pig. Spread upon a porous plate and exposed to sunlight it was found to be innocuous for inoculation after forty-eight hours. Cadéac gives other evidence of like kind, and comes to the conclusion that dust which will cause tuberculosis with so much difficulty by direct inoculation into the peritoneal cavity of a guinea-pig will have much more difficulty in infecting man's respiratory passages.

Behring, however, advocates the doctrine of contagion in regard to tuberculosis in a different manner. He believes that infection takes place in the main in infancy through the intestinal canal (the contagion being derived from milk), and that thereafter the infecting Bacilli, lodging in different parts of the body, commonly remain latent for years, perhaps for a long series of them. All this, however, is pure hypothesis. As I have said elsewhere: ${ }^{1}$ "He postulates long periods of latency after

1 "The Nature and Origin of Living Matter," 1905, p. 327. 
systemic infection, hard to be believed; and ultimately requires agencies for waking the tubercle Bacilli into activity, just such as I suppose may be adequate for calling them into being-namely, malnutrition and conditions of lowered vitality, howsoever produced: though among such factors impure air and inadequate or improper food must take an important place."

But in accordance with the view favoured by me, as to the frequent origin of the tubercle Bacillus by transformation of common Bacilli constantly gaining access to the body, we might return to something more like the doctrine "that prevailed concerning the etiology of phthisis only a few years ago, when the affection was freely recognised as generable in the individual, altogether apart from contagion, and contagion was supposed to take only a limited share in the production of the disease. This seems the more rational and most warranted view to take. It is one which would tend to lay stress upon the need for prevention as well as cure, but it would not encourage the view that the disease could be exterminated, or even very largely diminished, by the provision of 'sanatoriums' and by efforts to minimise the risk of contagion [-all important as these undoubtedly are]. I merely mean to imply that, in my opinion, contagion is as much over-rated as genesis is under-rated, and that our notions concern. ing prevention must not be too much centred upon the mere elimination of contagion." As Professor Hueppe says, "the resisting power of a population can change according to the social conditions. If 
through industrial development the social conditions become bad, we have always and everywhere an increase of tuberculosis, and this depends more on the increase of predisposition than on the increase of exposition. . . . It is impossible to successfully fight against tuberculosis if we only fight against the bacilli. . . . It is therefore absolutely necessary to increase the resisting power by means of positive hygiene, or through training one's self to health." 1

Bacteriologists, as a result of repeated processes of heating at brief intervals and often at high temperatures for the special purpose of bringing about complete sterilisation, not only kill all pre-existing micro-organisms, but, as I maintain, destroy also any potential germinality of the media themselves. These media, after having been treated as above indicated, will nourish and favour the multiplication of living Bacteria with which they are inoculated, but they will never engender them. 'They behave, in fact, exactly like a boiled ammonium tartrate solution - this also does not engender, but will freely nourish Bacteria." As a result of habitually dealing with media thus treated (though they know that, with the exception of the spores of Bacilli, all the microbes in their media may be destroyed by being boiled once only, for five minutes or less), bacteriologists have come to the conclusion that Bacteria never arise de novo.

1 The Joumal of State Medicine, Jany. 1904, p. 10.

2 See pp. 57 and 58, where the difference between "nourishing" and "generating" fluids has been more fully set forth. 
Hitherto they have shown not the least disposition to modify their methods, with a view to seek such results as I have obtained. The modification needed would be, of course, to heat their nourishing fluids (semi-solid media would not be favourable for the trial) only sufficiently to destroy pre-existing living things, and then to take suitable means, by the agency of heat or light, to favour the possible engendering of new living matter.

Their exclusive occupation with their own methods is unfortunate simply because it has so long had the effect of giving sanction to ultracontagionist doctrines. But, as I have pointed out, the prosecution of their own researches, by their own methods, is now tending rapidly to show that wider views are necessary, and that they must recognise as facts what for so long it has been their habit to deny-namely, that contagious and infectious diseases frequently arise de novo.

Still this view is being forced upon them independently of any necessary belief in the "spontaneous generation" of micro-organisms. It comes, as I have pointed out, as a consequence of multitudinous observations tending to show that pathogenic are constantly being derived, as Lehmann and Neumann say, from non-pathogenic organisms-by reason of gradual alterations brought about not so much in their form as in the metabolic processes inseparable from their life and growth.

When a contagious disease is once established, of course everything must be done, as far as possible, to check its course in the individual; and likewise 
to diminish, as far as possible, its spread to other members of the community. In both these directions the work of bacteriologists has been proving of incalculable value. Their study of toxins, antitoxins, opsonins, and serum-therapy generally is gradually leading to brilliant results both in the prevention and in the cure of disease. I, however, am naturally not so sanguine as many others, who still pin their faith to ultra-contagionist doctrines, that these diseases can be effectively stamped out by the mere prevention of contagion. Believing, as we all must, in their de novo origin in the past, I have always failed to see why they may not also be engendered in the present, and have been impressed by the weight of evidence tending to show that several of them, at least, do so arise from time to time. Modern researches are now justifying this view and lending support, as I urged five and thirty years ago, to the necessity for the most diligent search into the conditions of origin of all these affections. And the more contagious the disease, the more important does the quest become. 


\section{INDEX}

Abiogenesis, I

Acid urine, barren after boiling, $\mathrm{I}_{3} \mathrm{I}$ I 33

Acids, retard fermentation, I I6-I I 8

Actirium, I6, I7

Actinomyces, 255, 256

Adami, Prof., 306

Air of flasks replaced by other gases, 89

Airless flasks, 90-92, I 37 -I 45, 257

Alpha rays, 16

Ammonium tartrate solution, 57 ; growth of organisms in, 235. 312

Andrewes, Dr, 307

Aristotle, 4, 29

Atomic Theory, 14

Atoms, 4 ; complexity of, 8 ; constitution of, 7 ; disintegration of, IO, I 5 ; genesis of, 17

Atoms of hydrogen, 6 ; of mercury, 6

BACILli in superheated infusions, 196

Bacilli, spores of, 70 ; death-point of, 7 1-74; after desiccation, 75-86

Bacillus of Tubercle, 306, 308; of Typhoid, 301

Bacteria, as things of yesterday, 308 ; forms of, dependent on molecular composition, 308 ; growth of, in saline solutions, $230-282,235,3$ I 2 ; in air, 39-4I ; paucity of, 272

Bacteria, heterogenetic origin of, $285^{-}$ 288 ; in cells of potato, $285-287$; in cells of turnip, 286 ; in spines of Cyclops, 287

Bacteria, thermal death-point of, 5666 ; evidence of life of, 97

Bacteriological science, 296

Bacteriologists and their work, 3 I 2-3 I 4

Barry, De, 73

Bath of calcium chloride, 234; of colza oil, 234

Beer-wort, experiments with, 194

Behring, Prof., 3 Io

Bennett, Prof. Hughes, I 20

Bickerton, Prof., 3

Blaxall, Dr, 67
Boiling water and living matter, 4552 ; potency of, 259

Boussingault, M., ${ }_{15} 8$

Breeding true, 291, 29S

Brownian movements, 96

Buffon, xv, 87

Bulliard, 74

Burdach, xiv, 5I

Calcined air, 88

Calcium chloride bath, 234

Cathode rays, 5

Cliamberland, Ch., I75, I79, I8I, 208, 2.32

Chemical elements in stars, I2, I4

Child, Dr, 90

Classification of stars, I4

Cohn, Ferdinand, 54, 62, 70, 129, $214,227,228$

Colza oil bath, 234

Commission of Academy of Sciences, I 58 ; abortive ending of, 166 ; its constitution not approved, I6I : meeting of, I63-166; objections of M. Milne Edwards at, I63, I66; stipulations concerning proceedings of, I60

Complexity of atoms, 8 ; of matter, 8

Confervæe of hot springs, 53

Contagion of specific diseases, 298

Contagious diseases, their conditions of origin, 3 I 4

Convertibility of microbes, 300

Cooling, effects of different rates of, 276

Corpuscles, 6, I 8

Cotton wool, as plug for flasks, 89; flasks plugged with, I 45

Creative hypothesis, 26

Crookes, Sir Wm., 5

Crystalline form, constancy of, 290

Crystals and lowest organisms, 289 ; their forms independent of heredity, 290

Cucumber infusion, 194 


\section{DALTON, 5}

Darwin, 22, 26, 27, 3 I

Daylight, diffuse, influence of, 235237

Death-point, of Bacillus spores, 7I74 ; of Bacteria, 56-66 ; of Fungus spores, 65, 73; of Infusoria, 55 ; of Micrococci, Streptococci, and Staphylococci, 86 ; of seeds and eggs, 45-52 ; of Torulæe, 62

Debierne, I7

Democritus, 4

Diffuse daylight, influence of, 235-237

Diphtheria, origin of epidemic of, 302

Diphtheria and scarlet fever, 303

Discontinuous heating, 2 I 7

Discussion with M. Pasteur, I $5^{2-158}$

Disintegration of atoms, 15

Dissociated elements in stars, I 5

Distilled water, organisms in, 230

Duhamel, 46

Dumas, M., I 58-166

Duncan, Prof. Kennedy, I I, I3, I9

EBulditrion, sealing during, 9I

Eggs and seeds, thermal death-point of, $45-52$

Eidam, Dr, I 29

Electrons, 6, 13, I5, I8, 22

Elements, evolution of, 20,23

Emanation of radium, I6

Ether, I8, 22

Evolution hypothesis, 292, 296

Evolution, inorganic, IO, I9; of elements, 20 ; organic, 19

Experimental conditions, nature of, 36 ; disadvantages of, 37 ; fallacies to be guarded against, 37

Experimental results, general interpretation of, 279

Experimental tube, 233

Experiments concerning Archebiosis, 41 ; organisms found, 42

Experiments of Prof. Tyndall, 209. 228

Experiments with airless flasks, I37I45; with beer-wort, I94; with cucumber infusion, I95; with hay infusion, IOI-IO3; with milk, I95; with potato infusion, I95; with saline solutions, 230-282; new, with sodium silicate, $243^{-282}$; old, with sodium silicate, $24 \mathrm{I}$; with sea-water, 276 ; with turnip and cheese, Ioo-
I06; with urine, 193; with urine and liquor potassæ, I 30 - I 50

Explosive compounds, I 7

Fermentation caused by liquor potassa, I50; delayed by acids, I 16 I I8; favoured by potash, I I6-I I8; signs of, in superheated fluids, 196206 ; stimulated by oxygen, I 45

Fertility of boiled neutral fluids, cause of, I33-150, I67, I9I

Flasks plugged with cotton wool, 145

Fray, I 89

Fremy, I62, I65

Fungus germs, death-point of, 65,73

Generating fluids, $58,86,312$

Genesis of elements, 23 ; of living matter, 20, 23

Gerhardt, I I6, I 23

Germs in air, 39-41, 272 ; in water, $4 \mathrm{I}$; invisible, $35,5 \mathrm{I}, 70,28_{3}$; of fungi, death-point of, 65,73 ; of mould, 65

Gruithuisen, 63

HARMLESS organisms, 297, 306

Hankin and Yersin, 304

Hartley, IV. N., I I 2

Hay bacillus, spores of, 70 ; deathpoint of, 7 I-74, 75-86, 209

Hay infusion, experiments with, Ior, IO3; superheated, experiments with, 194

Helium, I6

Helmholtz, Prof., 24

Hemming, G. W., 59, I6I, 22 I-22S

Heredity, not operative in crystals, 290 ; nor in lowest organisms; 290, 307

Heterogenetic origin of Infusoria, 292 ; supporting evidence of, 292 ; of Bacteria, 2S5-2SS; motionless particles first appear, $2 S S$

Heterogenesis, xiv-xviii, 293

High incubating temperatures, I2S- I 30

Hoffmann, 74

Horwath, Dr, 62

Hot springs, conferve of, 53

Hueppe, Prof., 300, 3 II

Hueter, Prof., 299

Huizinga, Prof., IO7-I09, II4, I8I, I $S_{3}, 227,228$

Huxley, Prof., 28, 31, 33, 43, 96, I I0I I $5,208,228$ 
INCIPIENT MOULDS, 256, 264

Incredulity concerning my experimental results, 97-I IO

Incubating temperatures, high, I 28 - I 30

Inductions of Pasteur, 33

Infusion of cucumber, I95; of hay, IOI-IO3, I94; of potato, I95; of turnip, roo- Io6

Infusoria, ciliated, 292

Infusoria, thermal death-point of, 55 ; heterogenetic origin of, 292

Inhabited worlds, multitudes of, I

Inorganic evolution, I0, I9

Interpretation, general, of experimental results, $279-282$; of my experiments by Pasteur, I77-I 80 ; disproof of this explanation, I80189

JoLy and Musset, I 20

Joubert and Pasteur, I 56, I78, I 83 , I 87,23 I

KELvin, Lord, 24

Koch, Dr, 70

Kühne, Prof., 55

Le Bon, Gustave, I 8

Lehmann and Neumann, S5 $_{2}$ 255, 300, 304, 3 I 3

Leptothrix, I02-105, I 36

Leucippus, 4

Leuconostoc, 255

Liebig, Baron, 6 I

Life and boiling water, 45-52; mode of origin of, xvii, 35 ; notions concerning, $\mathrm{xv}$; origin of, 24,26

Lister, Lord, 77, I3I, I84, I88

Living matter, origin of, 20,26 ; in multiple sites and times, $2 \mathrm{I} ;$ as minute specks, 30 ; its growth in ammonium tartrate solutions, 284 ; different organisms growing side by side, 285 ; proclivity to formation of, 284

Liquor potasse and urine, experiments with, I $30-150$; by M. Pasteur, I73176; by Prof. 'Tyndall, I 5I-I53; by Sir Wm. Roberts, I 5 I-I 53 ; excess of liquor potassæ harmful, I 49 ; interpretation of results, I 47 - I 50

Liquor potassæ, no germs in, 186

Liquor potassæ tubes, I37-I 40 ; superheating of, 152 ; supposed to contain living germs, I 48 , I $54^{-1} 5^{8}$
Lockyer, Sir Norman, 3, IO, I 2, I 4

Loculated Micrococci, 254

Lodge, Sir Oliver, 7

Lowest organisms, present day existence of, 293

Lumps, protective influence of, I IO

M'CALL, Mr, I I I, I I 4

Macfadyen, Dr Allan, 67, 235, 272

Mackeown, Dr, 304

$\mathrm{M}$ 'Weeney, Dr, 302

Mantegazza, Prof., 89

Matter, complexity of, 8

Max-Schultze, 53, 55

Microbes, convertibility of, 300

Micrococci, filaments from, 27I-273; in loculi, 254, 27I-273; in milk, 206 ; in superheated infusions, 196 ; in unboiled urine, I84, 187-I89; origin of, 209

Micro-organisms, bodies simulating, 247 ; death-point of, 229, 280-282; proof of their de nozio origin, 280 282 ; variability of, 29 I

Milk, superheated, changes in, 205

Mill, John Stuart, 30

Milne Edwards, I 58, I6I-I63, I66

Minute specks, origin of living matter as, 30, 35

Misrepresentations of author's views, 296

Molecular complexity of protoplasm, $29 \mathrm{I}$

Moseley, H. M., 97

Mould, in solutions of silica, 240; spores of, death-point of, 65

NATURAL selection and heredity, 397

Nature, uniformity of, 28

Needham, Turberville, xv, 44, 87,90

Neutral fluids, cause of fertility of, 133-150, 167; death-point of germs in, $120-127$

Newcomb, Prof. Simon, I

Newman, Dr George, 259

Non-nucleated organisms and heredity, 307

Note by M. Pasteur, 157

Nourishing fluids, 58,86

OlD hay germs, 78, 219

Omne vivum ex vivo, 30

Optimun temperature, I 28

Organic evolution, 19, 22 
Organisms and crystals, 289

Organisms, growih of, in ammonium tartrate solutions, 235

Origin of Infusoria by heterogenesis, 292

Origin of life, 20, 24; in multiple sites and times, $2 \mathbf{I}$; in other worlds, 22

Origin of plague, 304 ; of septicæmia, 301 ; of tuhercle bacillus, 306, 308 , 3 I ; of living things, xvii

Ostwald, Prof., 278

Oxygen, stimulating fermentation, I 45

PARK, Dr W. II., 259, 282

Pasteur, Louis, 3I, 32, 33, 44, 58, 59, S9, 95, I I 8-127, 131, 133, I 57, I68I70, I73-I80, I 83, I87, I88, 2 I 2 , $227,231,285$

Pasteur and Joubert, 156

Pasteur's solution, 57

Pathogenic from non-pathogenic organisms, 300, 306

Peptone replacing cheese, Io8

Pest, 304

Phthisical sputa, 30S-j10

Phthisis, changes of doctrine concerning, 308 ; spread of, by contagion only, 308 ; evidence against this view, $30 S-3$ I 2

Pickering, Prof., I4

P'lague, possible mode of origin of, 304

Planets, are they inhabited, 2, 25

Platinum electrodes in flasks, I 45

Potash favouring fermentation, i i6I 18

Potency of boiling water, 259

Pouchet, M., xv, 55, 89, 90

Procter, R. A., 2

Proto-elements, I4

Protoplasms, molecular complexity of, 29 I ; unity of, 43

Protyle, 5, I 5

Pure urine, appearance of bacilli in, I88; difterent micro-organisms in, almost at will, IS9; experiments with, I 84, I $\$ 7$ - I 89

Quincke, Prof., 275

RaIIUM, 8, I6 ; emanation of, I6

Ramsay, Sir WWm., I6, 235

Rats, as spreaders of plague, 305
Revelations of spectroscope, 3, Io

Roberts, Sir Wm., I32, I5I-I53, I 67

Roberts, Sir W. Chandler, 240, 278

Robin, Charles, I65, I 70

Rutherford, Prof., I6

SaCCharomyces, living at high temperature, 229

Saline solutions, effects of superheating, 247, 274, 276; experiments with, 230-2S2; heated to $100^{\circ} \mathrm{C} ., 255$; heated to $\operatorname{I} 15^{\circ} \mathrm{C}$, $26 \mathrm{I}-264$; heated to $\mathrm{I} 20^{\circ} \mathrm{C}$, 264 ; heated to $125^{\circ}$ C., $265-267$; heated to $130^{\circ} \mathrm{C} ., 267-274$

Sanderson, Burdon, 57, 75, 98-107, I08, I09, I78, I81, I S2, 227, 228

Sarcinæ, 272

Scarlet fever and diphtheria, 303

Schwann, 88, 90, I I9, I 20

Sealed vessels, safety of, $3^{8}$

Sealing during ebullition, 9 I

Sea-water, experiments with, 276

Seeds and eggrs, thermal death-point of, $45-52$

Septicæmia, origin of, $30 \mathrm{I}$

Silica, effects of superheating, 266, 274 ; and change of in uviol tubes, 266, 275

Silicate solutions, composition of, 249 25I; effects from superheating, 274; examination of, 244-246; growth of mould in, 240 ; microorganisms found in, 252-255; preparation of, 243

Silicon, abundance of, in earth's crust, 278 ; as substitute for carbon, 239 242,277

Simplest kind of protoplasm, 236

Soddy, F., I 6

Sodium silicate, new experinents with, 243-282; old experinients with, 24 I

Spallanzani, Abbé, experiments of, $44-52,87$, I 19

Specific diseases and contagion, 298

Specific from common microbes, 305

Specific microbes, origin of 29S-300

Spectra of elements varying, I 2

Spectral lines of stars, 3 , I3

Spectroscope, II ; revelations of, 3 , IO, 23

Spectrum analysis, I I

Spencer, Herbert, 27, 28, 31 
Spontaneous generation, xiii, 29, 3I, $32,44,298$

Spores of Bacilli, 70, 220, 232 ; deathpoint of, 7I-74; after desiccation, $75-86$

Spores of Fungi, 74 ; of mould, deathpoint of, 65,73

Staphylococci in hay infusion, I 89 ; in sea-water, 277

Stars, chemical elements in, I2, I4; classification of, I4; dissociated elements in, I5; spectral lines of, 3,13

Sterilising temperature, 26I

Stokes, Sir Geo., 2, 24

Stoney, Dr Johnstone, 6

Streptococci, evolution of, 307 ; in capsules, 255 ; in hay infusion, 204 ; in pure urine, I89; in sea-water, 277 ; supposed long line of ancestors of, 307

Superheated fluids, signs of fermentation in, 196-206; potash tubes, I 52 ; retorts, first experiments with, IO5; are the latter necessary, I $82-\mathrm{I} 85$

Superheating, effects of, 247,274

Survival of germs, 44, I32, I 92

TARNOWSKI, 65

Temperature of ebullition in vessels with capillary orifices, I I 2

Thermal death point of Bacteria, $5^{6}$ 66 ; of Fungus-germs, 65, 73; of Infusoria, 55; of seeds and eggs, 45-52 ; of Torulæ, 62

Thermophilic Bacteria, 67-69, 85, 28I ; not found in tap-water, 69,85

Thomson, Prof. J. J., I I

Thorium, 8, I6

Thresh and Porter, Drs, 259

Tinned meats and vegetables, preparation of, I II-II 3
Tonsillitis, 303

Torulæe, 269; death-point of, 62 ; light favouring appearance of, 253 ; multiplying, 274

Torula-threads, 268

Tubercle bacillus, its origin, 306,308 ; supposed long line of ancestors, 306

Turner, Prof. H. H., 24

Turnip and cheese experiments, IooI06, 107

Tutton, A. E. H., 290

Tyndall, Prof., 29, 32, 44, 58, 78, 92, I 67, I 70, I $87,209-228$

Typhoid bacilli, 30 I

ULTRA-CONTAGIONIST views, 297, 305

Uniformity of nature, 28

Unity of protoplasm, 43

Uranium, 8, I6

Urea, decomposition of, by boiling, I68-170 ; in incubator, $170-173$

Urine, pure, experiments with, I 84 , I 87 - I 89 :

Urine, superheated, experiments with, I.93

Urine and liquor potassee experiments, I 30-I 50; excess of liquor potassa harmful, I49; repeated by M. Pasteur, I 73-I 76 ; by Prof. Tyndall, I 5 I-I 53, I 70 , I 76 ; by Sir Wm. Roberts, I 5 I-I 53

Van Tieghem, I 65

Variability of microbes, 29I

Vibriones, 270

WATER, bacteria in, 4I

Wildermann, 238

Wöhler, Prof., 240

Worlds, inhabited, multitudes of, I

Wurtz, Prof., I65

Wyman, Prof. Jeffries, 53, 54, 8S, I 20 
PRINTED BY

TUKNBULL AND SPEARS,

EDINBURGH 


\section{A CATAlogue OF BOOKS}

\section{PUBLISHED BY METHUEN}

AND COMPANY: LONDON

\section{ESSEX STREET}

W.C.

\section{CONTEN TS}

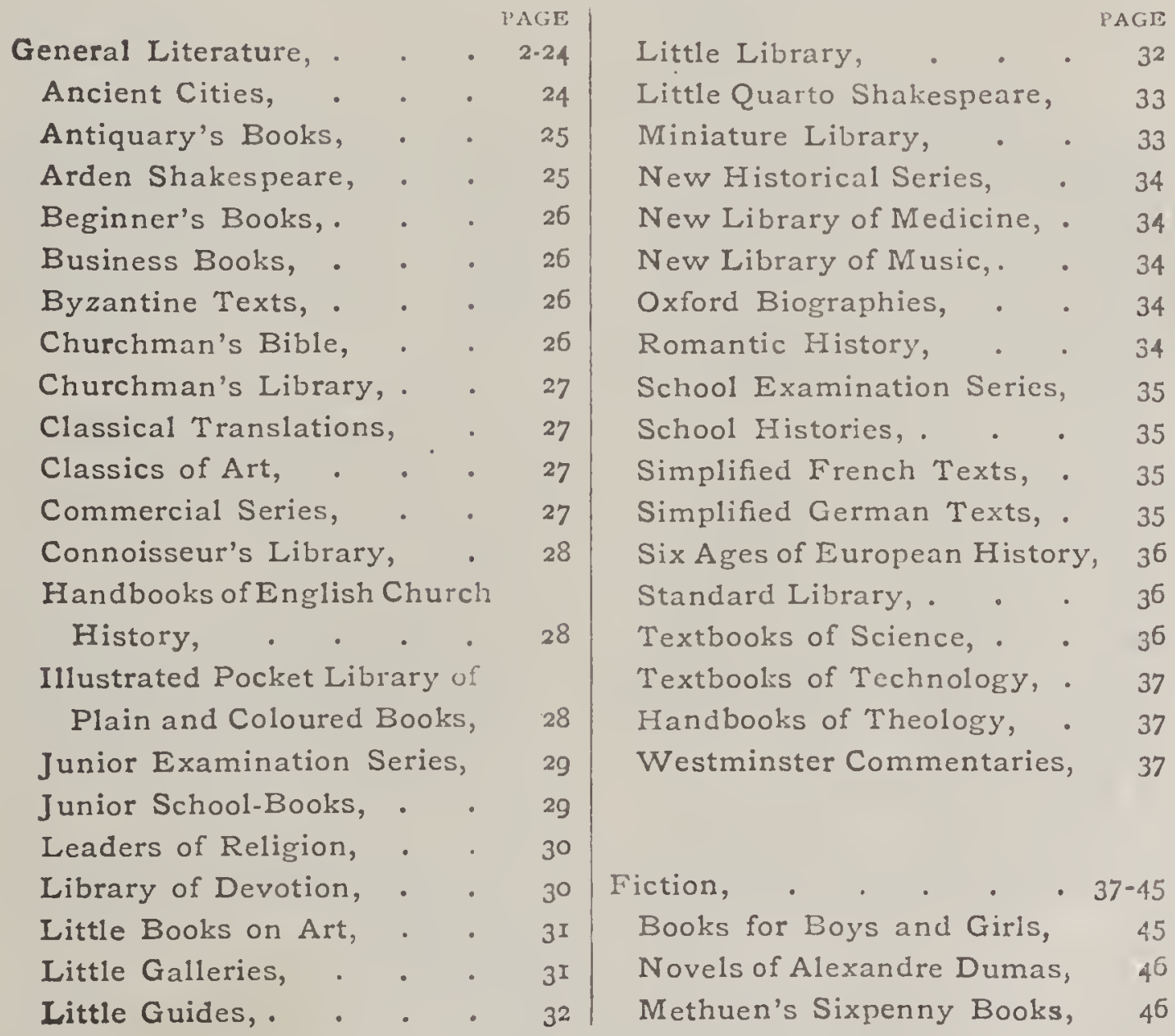




\section{A CATAlogue OF}

\section{MESSRS. METHUEN'S}

\section{PUBLICATIONS}

In this Catalogue the order is according to authors. An asterisk denotes that the book is in the press.

Colonial Editions are published of all Messrs. Methuen's Novels issued at a price above $2 s .6 d$, and similar editions are published of some works of General Literature. Colonial editions are only for circulation in the British Colonies and India.

All books marked net are not subject to discount, and cannot be bought at less than the published price. Books not marked net are subject to the discount which the bookseller allows.

Messrs. METHUEN's books are kept in stock by all good booksellers. If there is any difficulty in seeing copies, Messrs. Methuen will be very glad to have early information, and specimen copies of any books will be sent on receipt of the published price plus postage for net books, and of the published price for ordinary books.

I.P.L. represents Illustrated Pocket Library.

\section{Part I. - General Literature}

Abraham (George D.) THE COMPLETE MOUNTAINEER. With 75 Illustrations. Second Eidition. Demiy 8vo. I5s. net.

Acatos (M. J.). See Junior School Books.

Addleshaw (Percy). SIR PHILIP SIDNEY. With 12 Illustrations. Demy 8vo. Ios. 6d, net.

Adeney (W. F.). M.A. See Bennett (IV. H.) Ady (Cecilia M.). A HISTORY OF MILAN UNDER THE SFORZA. With 20 Illustratious and a Map. Demy 8ro. Ios. 6i. net.

Aeschylus. See Classical Translations.

Ainsworth (IV. Harrison). See I. P.L.

Aldis (Janet). THE QUEE N O F l,ETTER WRITERS, MAROUISE IE Sévigné, Dame DE Bourbilly, 1626-96. With is Illustrations. Second Edition. Demy 8vo. iss. 6d. net.

Alexander (William), D.D., Archbishop of Armagh. 'THOUGH'T S A D COUNSELS OF MANY YEARS. Demy I6mo. 2s. 6 2 .

Alken (Henry). See I.P.L.

Allen (Charles C.). See Textbooks of Technology.

Allen (L. Jessie). See Little Books on Art.

Allen (J. Romilly), F.S.A. See Antiquary's Books.

Almack (E.), F.S.A. See Little Books on Art.

Amherst (Lady). A S I ETCH OF EGYPTIAN HISTORY FROM THE EARLIEST TIMES TO THE PRE. SENT DAY. With many Illustrations and Maps. A New and Cheaper Issue Demy 8vo. 7s. 6d. net.

Anderson (F. M.). THE STORY OF THE BRITISH EMPIRE FOR CHILDREN. With 42 Illustrations. Cr. 8v0. 2s.
Anderson (J. G.), B.A., NOUVELLE GRAMIMAIRE FRANCAAISE, A L'USAGE DES ÉCOlES ANGlaises. Crown $8 \%$. 25 .

EXERCICES DE GRAMMAIRE FRAN CAISE. Cr. 8vo. is. $6 d$.

Andrewes (Bishop). PRECES PRI VATAE. Translated and edited, with Notes, by F. E. BrightMan. M.A., of Pusey House, Oxford. Cr. 8zo. 6s. See also Library of Devotion.

'Anglo=Australian.' AFTER-GLOW MEMIORIES. Cr. Szio. 6s.

Anon. THE BUDGET, THE LAND AND THE PEOPLE. Second Edition. Crown 8vo. 6d. net.

HEALTH, WEALTH, AND IVISDOM. Crown 8z'o. Is. net.

THE IVESTMIINSTER PROBLEMS BOOK. Prose and Verse. Compiled from The Saturday IVestminster Gazette Com. petitions, 1004-1007. Cr. 8vo. 3s.6d. net.

VENICE AND HER TREASURES. With many Illustrations. Round corners. Fcap. 8io. 5s. net.

Aristotle. THE ETHICS OF. Edited, with an Introduction and Notes by Jorn Burnet, M.A., Cheaperissue. Demy8vo. Ios. 6i. net.

Asman (H. N.), M.A., 13.D. See Junior School Books.

Atkins (H. G.). See Oxford Biographies.

Atkinson (C. M.). JEREMY BENTHAM. Demy 8vo. 5s. net.

Atkinson (C. T.), M.A., Fellow of Exeter College, Oxford, sometime Demy of Magdalen College. A HISTORY OF GER. MANY, froin 1713 to $1 \delta_{15}$. With 35 Maps and Plans Derny 8vo. I5s. net. 
Atkinson (T. D.). ENGLISH ARCHITECTURE. With I96 Illustrations. Fcap. 8vo. 3s. 6d. net.

A GLOSSARY OF TERMS USED IN ENGLISH ARCHITECTURE. With 265 Illustrations. Second Edition. Ficap. 8vo. 3 s. 6d. net.

Atteridge (A. H.). NAPOLEON'S BROTHER S. With 24 Illustrations. Demy 8vo. I8s. net.

Auden (T.), M.A., F.S.A. Sec Ancient Cities.

Aurelius (Marcus). WORDS OF THE ANCIENT WISE. Thoughts from Epictetus and Marcus Aurelius. Edited by W. H. D. Rouse, M.A., Litt. D. Fcep. 8vo. 3s. 6d. net.

See also Standard Library.

Austen (Jane). See Standard Library, Little Library and Mitton (G. E.).

Aves (Ernest). CO.OPERATIVE IN DUSTRY. Crown 8vo. 5s. net.

Bacon (Francis). See Standard Library and Little Library.

Bagot (Richard). THE LAKES OF NOR'THERN ITALY. With 37 Illustrations and a Map. Fcap. 8vo. 5s.net.

Bailey (J. C.), M.A. See Cowper (W.).

*Bain (R. Nisbet). THE LAST KING OF POLAND AND HIS CONTEMPORARIES. With r6 Illustrations. Demy 8vo. Ios. $6 d$. net.

Baker (W. G.), M.A. See Junior Examination Series.

Baker (Julian L.), F.I.C., F.C.S. See Books on Business.

Balfour (Graham). THE L I FE O F ROBERT LOUIS STEVENSON. With a Portrait. Fourth Edition in one Volume. Cr. 8vo. Buckran, 6s.

Ballard (A.), B.A., LL.D. See Antiquary's Books.

Bally (S. E.). See Commercial Series.

Barham (R. H.). See Little Library.

Baring (The Hon. Maurice). WITH THE RUSSIANS IN MANCHURIA. Third Edition. Demy 8vo. 7s. 6d. net.

A YEAR IN RUSSIA. Second Edition. Demy 8vo. ros. 6d.net.

RUSSIAN ESSAYS AND STORIES Second Edition. Cr.8vo. 5s. net. Also published in a Colonial Edition.

Baring =Gould (S.). THE LIFE OF NAPOLEON BONAPARTE. Withnearly 200 Illustrations, including a Photogravure Frontispiece. Second Edition. Wide Royal 8vo. 10s. 6d. net.

THE TRAGEDY OF THE CESARS A Study of THE Characters of THE Casars of the Julian and Claudian HousES. With numerous Illustrations from Busts, Gems, Cameos, etc. Sixth Edition. Royal 8vo. Ios. 6d. net.

A BOOK OF FA I R Y TALES. With numerous Illustrations by A.J. GASkiN.
Second Edition. Cr. 8\%o. Buckram. 6s., also Medium 8vo. $6 d$.

OLD ENGLISH FAIRY TALES. With numerous Illustrations by F. D. BEDFORD. Third Edition. Cr.8vo. Buckram. 6s.

THE VICAR OF MORWENSTOW. Revised Edition. With a Portrait. Third Edition. Cr. 8ro. 3s. 6 .

OLD COUNTRY LIFE. With 69 Illustrations. Fifth Edition. Large Crown 8vo. $6 \mathrm{~s}$.

A GARLAND OF COUNTRY SONG: English Folk Songs with their Traditional Melodies. Collected and arranged by $\mathrm{S}$. Baring-Gould and H. F. Sherpari. Demy 4 to. $6 s$.

SONGS OF THE WEST: Folk Songs of Devon and Cornwall. Collected from the Mouths of the People. ByS. BARING-Gould, M.A., and H. FleETwood SHEPPARD, M.A. New and Revised Edition, under the musical editorship of CECIL J. SHARP. Large Imperial 8\%o. 5s. net.

A BOOK OF NURSERY SONGS AND RHYMES. Edited by S. BARING-Gould. Illustrated. Second and Cheaper Edition. Large Cr. 8vo. 2s. 6d. net.

STRANGE SURVIVALS: SOME CHAPTERS IN THE History of MAN. Illustrated. Third Edition. Cr. 8zo. 2s. 6d.net.

YORKSHIRE ODDITIES : INCIDENTS And Strange Events. Fifth Edition. Cr. 8vo. 2s. 6\%. net.

THE BARING-GOULD SELECTION READER, Arranged by G. H. Rose. Illustrated. Crowen 820. Is. $6 d$.

THE' BARING-GOULD CONTINUOUS READER. Arranged by G. H. Rose. Illustrated. Crown 8vo. Is. 6d.

A BOOK OF CORNWALL. With 33 Illustrations. Second Edition. Cr. 8vo.6s.

A BOOK OF DARTMOOR. With 6o Illustrations. Second Edition. Cr. 8vo. $6 s$.

A BOOK OF DEVON. With 35 Illustrations. Thurd Edition. Cr.8vo. 6s.

$\triangle$ BOOK OF NORTH WALES. With 49 Illustrations. Cr. $8 v 0.6 s$.

A BOOK OF SOUTH WALES. With 57 Illustrations. $\mathrm{Cr} .820 .6 \mathrm{~s}$.

A BOOK OF BRITTANY. With 69 Illustrations. Second Edition Cr.8vo. $6 s$.

A BOOK OF THE RHINE: From Cleve to Mainz. With 8 Illustrations in Colour by TREvor HADDEN, and 48 other Illus. trations. Second Edition. Cr. 8zo. 6s.

A BOOK OF THE RIVIERA. With 40 Illustrations. Cr. 8vo. $6 s$.

A BOOK OF THE PYRENEES. With 25 Illustrations. $C r .8 v 0.6 s$.

See also Little Guides.

Barker (Aldred F.). See Textbooks of Technology.

Barker (E.), M.A. (Late) Fellow of Merton College, Oxford. THE POLITICAL THOUGHT OF PLATO AND ARISTOTLE. Demy 8zo. 10.s. 6d. net.

Barnes (W. E.), D.D. See Churchman's Bible. 
Barnett (Mrs. P. A.). Sce Little Library. Baron(R. R. N.), M.A. I'RENCH PROSE COMIPOSITION. Fourth Edition. Cr. 8vo. 2s. 6a. Key, 3 s. net.

See also Junior School Books.

Barron (H. M.), M.A., Watham College, Oxford. TEXTS FOR SERMONS. With a Preface by Canon Scott Holland. Cr. 8vo. 35.6 \%.

Bartholomew (J. G.), F.R.S.E See Robertson (C. G.).

Bastable (C. F.), LL.D. THE CONMIERCE OF NATIONS. Fourth Ed. Cr. 8vo. 2s. 6d.

Bastian (H. Charlton), M.A., M.D., F.R.S. THE EVOLUTION OF LIFE. With Diagrams and many Photomicrographs. Demy 8vo. 7s. 6d. net.

Batson (Mrs. Stephen). A CONCISE HANDBOOK OF GARDEN FLOWERS. Ficap. 8\%0. $35.6 \%$.

THE SUMNER GARDEN OF PLEASURE. With 36 Illustrations in Colour by Osmund Pittman. Wide Demy" 8vo. I5s. net.

Bayley (R. Child). THE CONPLETE PHOTOCRAPHER. With over Ioo Illustrations. With Note on Direct Colour Process. Third Edition. Demy 8 vo. Ios. 6 d. net.

Beard (W. S.). EASY EXERCISES IN ALGEBRA FOR IBEGINNERS. Cr. Svo. Is. $6 d$. With Answers. 1s. gd.

See also Junior Examination Series and Beginner's Books.

Beckett(Arthur). ITHE SPIRIT OF THE DOWNS : Impressions and Reminiscences of the Sussex Downs. Witl zo Illustrations in Colour by Stanley Inchbold. Demy 8zio. Ios. 6\%. net.

Beckford (Peter). TH OUGH T S ON HUNTING. Edited by J. Otho Paget, and Illustrated by G. H. JALland. Second Edition. Denty 8zio. 6s.

Beckford (William). See Little Library.

Beeching (H. C.), M.A., Caun of Vestminster. See Library of hevotion.

Beerbolim (Max). A bOOK OF CARI CATURES. Imporial to. 2rs. net.

Begbie (Harold). MISTER WORKERS. Illustrated. Demy 8vo. 75.6 . net.

Behmen (Jacob). DIALOGUES ON THE. SUPERSENSUAL LIFE. Edited by Bernard Holland. Ficap. 8*0. $35.6 \mathrm{~d}$.

Bell (Mrs. Arthur G.). THE SKIRTS OF THE GREAT CITY. With I6 Illustrations in Colour by ARTHuR G. BELL, I7 other Illustrations, and a Map. Second Edition. Cr.8vo. 6s.

Belloc (H.) PARIS. With 7 Maps and a Frontispiece in Photogravure. Second Ei $i$ tion, Revised. Cr. 8vo. $6 s$.

HILLS AND THE SEA. Second Edition. Crowen 8vo. 6s.

ON NOTIING AND KINDRED SUIBJECTS. Second fidition. ficap. 8vo. $5 s$. \# ON TiVHRYTHING. Ficas. 8\%' 5 s.

MARIE ANTOINET'TL. Wit 35 Portraits and Illustrations, and 22 Maps. Demy 8vo. I5s. nct.

THE PYRENEES. With 46 Sketches by the Author, and 22 Maps. Second Edition. Demy 8ro. 75. 6d. net.

Bellot (H.H.L.), M.A. See Jones (L. A.A.)

Bennett (Joseph). FORTY IEARS OF MUSIC, I865-1905. With 24 Illustrations. Demy 8vo. I6s. net.

Bennett (W. H.), M.A. A PRIMER OF THE BIBLE. Fifth Edition. Cr. 8io. 2s. $6 d$.

Bennett (W. H.) and Adeney (W. F.). A BIBLICAL INTRODUCTION. With a concise Bibliography. Fift/z Edition. Cr. 8vo. 7s. 6\%

Benson (Archbishop) GOD'S BOARD. Communion Addresses. Second Edition. Fcap. 8zo. 3.s. 6d. net.

Benson (A. C.), M.A. See Oxford Bio. graphies.

Benson (R. M.). THE IVAY OF HOLI NESS. An Exposition of Psalm crix. Analytical and Devotional. Cr. 8ro. $5 s$.

Bernard (E. R.), M.A., Canon of Salisbury THE ENGLISH SUNDAY: ITS ORIGINS AND ITS Claims. Fcap.8vo. is. $6 d$.

Berry (W. Grinton), M.A. FRANCE SINCE WATERLOO. With I6 Illustra. tions and Maps. Cr. 8ro. 6s.

Beruete (A. de). Sce Classics of Art.

Betham-Edwards (Miss). HOME LIFE IN FRANCE. With 20 Illustrations. Fifth Edition. Crowus 8vo. $6 s$.

Bethune-Baker (J. F.), MI.A. See Handbooks of Theology.

Bindley (T. Herbert), D.D. THE OECU. MENICAL DOCUMENTS OF THE FAITH. With Introductions and Notes. Second Edition. Cr. 8vo. 6s. net.

Binns (H. B.). THE LIFE OF IVALT WHITMAN. Illustrated. Demy Sio. Ias. $6 d$. net.

Binyon(Mrs. Laurence). NINETEENTH CENTURY PKOSE. Selected and arranged by. Crovent 8vo. $6 s$.

Binyon (Laurence). THE DEATH OF ADAM AND OTHER POEMIS. Cr. SZO. 3s. 6 d. net.

See also Blake (IVillian).

Birch (Walter de Gray), LL.D., F.S.A. See Connoisseur's Library.

Birnstingl(Ethel). See Little Books on Art. Blackmantle(Bernard). See I.P.L.

Blair (Robert). See I.P.L.

Blake (Villiam). THE LETTERS OF IVILLIAM BLAKE, TOGETHER WITH A LIFE BY FREDERICK TATHAM. Edited from the Original Manuscripts, with an Introduction and Notes, by ARchibald G. B. Russell. With i2 Illustrations. Demv 8:o. 7s. 6d. net.

ILIUSTRATIONS OF THE BOOK OF JOI). With General Introduction by LAURBNCE liInyon. Jureto. 2is. net. See alsu I.P.L., and Little Library. 
Bloom (J. Harvey), M.A. See Antiquary's Books.

Blouet (Henri). See Beginner's Books.

Boardman (T. H.), M.A. See French (W.).

Bode (Wilhelm), Ph.D. See Classics of Art.

Bodley (J.E. C.) THE CORONATION OF EDWARD VII. Demy 8vo. 21s. net. By Command of the King.

Body (George), D.D. TH E SOUL'S PILGRIMAGE: Devotional Readings from the Published and Unpublished writings of George Body, D.D. Selected and arranged by J. H. BurN, B.D., F.R.S.E. Demy I6mo. 2s. 6 d.

Bona (Cardinal). See Library of Devotion.

Bonnor (Mary L.). See Little Books on Art.

Boon (F. C.)., B.A. See Commercial Series. Borrow (George). See Little Library.

Bos (J. Ritzema). AGRICULTURAL ZOOLOGY. Translated by J. R. AinswORTH DAvis, M.A. With I 55 lllustrations. Second Edition. Cr. 8vo. 3s. 6d.

Botting (C.G.), B.A. E A S Y GR E E K EXERCISES. Cr. 8vo. $2 s$.

See also Junior Examination Series.

Boulting (W.) TASSO AND HIS TIMES. With 24 Illustrations. Demy 8vo. Ios. $6 d$. net.

Boulton (E. S.), M.A. GEOMETRY ON MODERN LINES. Cr. 8vo. $2 s$.

Boulton (William B.). SIR JOSHUA REYNOLDS, P.R.A. With 49 Illustrations. Second Edition. Demy8vo. 7s.6a. net.

Bovill (W. B. Forster). HUNGARY AND THE HUNGARIANS. With I6 Illustrations in Colour by WILLIAM PASCOE, I2 other Illustrations and a Map. Demy 8vo. 7 s. 6d. net.

Bowden (E. M.). THE IMITATION OF BUDDHA : Being Quotations from Buddhist Literature for each Day in the Year. Fifth Edition. Cr. I6mo. 2s. 6d.

Bower (E.), B.A. See New Historical Series.

Boyle (W.). CHRISTMAS AT THE ZOO. With Verses by W. Boyle and 24 Coloured Pictures by H. B. NeILson. Super Royal I6mo. 2s.

Brabant (F. G.), M.A. RAMBLES IN SUSSEX. With 30 Illustrations. Crown 8vo. 6s. See also Little Guides.

Bradley (A. G.). ROUND ABOUT WILT. SHIRE. With I4 Illustrations, in Colour by T. C. Gотсн, I6 other Illustrations, and a Map. Second Edition. Cr. 8vo. 6s.

THE ROMANCE OF NORTHUMBERLAND. With I6 Illustrations in Colour by Frant SoUTHGate, R.B.A., and I2 from Photographs. Second Edition. Demy 8vo. $7 s .6 d$ net.

Bradley (John W.). See Little Books on Art.

Braid (James), Open Champion, I90I, Ig05 and 1906. ADVANCED GOLF. With 88 Photographs and Diagrams. Fifth Edition. Denry 8vo. Ios. 6d. net.
Braid (James) and others. GREAT GOLFERS IN THE MAKING. Edited by HENRY LEACH. With 24 Illustrations. Second Edition. Demy 8vo. 7s. 6d. net.

Brailsford (H. N.). MACEDO N I A : ITS RACES AND THEIR FUTURE. With 32 Illustrations and 2 Maps. Demy 8vo. I2s. $6 d$. net.

Brentano (C.). See Simpllfied German Texts.

Brightman (F. E.), M.A. See Andrewes (Lancelot).

*Brock (A. Clutton). SHELLEY : $\Gamma \mathrm{HE}$ MAN AND THE POET. With I2 Illus. trations. Demy 8vo. 7s. 6d. net.

Brodrick (Mary) and Morton (A. Ander = son). A CONCISE DICTIONARY OF EGYPTIAN ARCH $\& O L O G Y . A$ A HandBook for Students and Travellers. With 80 Illustrations and many Cartouches. $\mathrm{Cr}$. 8vo. $3 s .6 d^{\prime}$.

Brooks (E. E.), B.Sc. (Lond.), Leicester Municipal Technical School, and James (W.H.N.), A.M.I.E.E., A.R.C.Sc., Municipal School of Technology, Manchester. See Textbooks of Technology.

Brown (S. E.), M.A., B.Sc., Senior Science Master at Uppingham. A PRACTICAL CHEMISTRY NOTE-BOOK FOR MATRICULATION AND ARMY CAN. DIDATES. Easy Experiments on the Commoner Substances. Cr. 4to. Is. 6d.net.

Brown (J. Wood), M.A. THE BUILDERS OF FLORENCE. With 74 Illustrations by Herbert Railton. Demy 4to. x8s.net.

Browne (Sir Thomas). See Standard Library.

Browneli (C. L.). THE HEART OF JAPAN. Illustrated. Third Editior. Cr. 8vo. 6s. Also Medium 8wo. 6\%.

Browning (Robert). See Little Library.

Bryant (Walter W.), B.A., F.R.A.S., F.R. Met. Soc., of the Royal Observatory, Greenwich. A HISTORY OF ASTRONOMY. With 47 Illustrations. Demy8vo. 75.6 d.net.

Buckland (Francis T.)!! CURIOSITIES OF NATURAL HISTORY. Illustrated by H. B. NeILson. Cr. 8vo. 3s. 6d.

Buckton (A. M.) THE BURDEN OF ENGEIA. Second Edition. Cr. 8ro. $3 s$. 6 . net.

EAGER HEART: A Mystery Play. Seventh Edition. Cr. 8vo. Is. net.

KINGS IN BABYLON : A Drama. Cr.8zo. Is. net.

SONGS OF JOY. $\mathrm{Cr}$, 8vo. is, net.

Budge (E. A. Wallis). THE GODS OF THE EGYPTIANS. With over Ioo Coloured Plates and many Illustrations. Truo Volumes. Royal 8vo. f, 3,3 . net.

* Buist Massac (H.). THE COM PLETE AERONAUT. With many Illustrations. Demy 8vo. x2s. 6d. net.

Bull (Paul), Army Chaplain. GOD AND OUR SOLDIERS. Second Editior. Cr. 8vo. 6s. 
Bulley (Miss). See Dilke (Lady).

Bunyan (John). THE PILGRIM'S PRO. GRESS. Edited, with an Introduction by C. H. FirTh, M.A. With 39 Illustrations by R. Anning Bell. Crozen 8vo. $6 s$. See also Standard Library and Library of Devotion.

Burch (G.J.), M.A., F.R.S. A MANUAL OF ELECTRICAL SCIENCE. Illustrated. $C r .8 \% o$. $3 s$.

Burgess (Gelett). GOOPS AND HOW TO BE THEM. Illustrated. Small 4 to. $6 s$.

Burke (Edmund). See Standard Library.

Burn (A. E.), D.D., Rector of Handsworth and Prebendary of Lichfield. See Handbooks of Theology.

Burn (J. H.) B. D. F. R.S. E. THE CHURCH M A 'S TREASURY OF SONG: Gathered from the Christian poetry of all ages. Edited by. Fcap. 8vo 3 s. 6 d. net. See also Library of Devotion.

Burnet (John), M.A. See Aristotle.

Burns (Robert), THE POEMS. Edited by ANdreiv Lang and W. A. Craigie. With Portrait. Third Edition. Wide Demy 8vo, gilt top. $6 \mathrm{~s}$.

See also Standard Library.

Burnside (W. F.), M.A. OLD TESTA MENT HISTORY FOR USE IN SCHOOLS. Third Edition. Cr. 8vo. $3 s .6 d$. Burton (Alfred). See I.P.L.

Bury (J. B.), M.A., Litt. D. See Gibbon (itdward).

Bussell (F. W.), D.D. C H R I S T I N THEOLOGY AND SOCIAL PROGRESS (The Bampton Lectures of I905). Demy' 8vo. Ios. 6d. net.

Butler (Joseph), D.D. See Standard Library.

Butlin (F. M.). AMONG THE DANES. With 12 Illustrations in Colour by ELLEN Wilkinson, and I5 from Photographs. Demy 8\%o. 7s. 6d. net.

Cain (Georges), Curator of the Carnavalet Museum, Paris. WALKS IN PARIS. Translated by A. R. Ai.linson, M.A. With a Frontispiece in Colour by Maxwer ARmiELD, and Ii 8 other Illustrations. Deny 8vo. 7s. 6i. net.

Caldecott (Alfred), D.D. See Handbooks of 'Theology.

Calderwood (D. S.), Headmaster of the Normal School, Edinburgh. TEST CARDS IN EUCLID AND ALGEBRA. In three packets of 40, with Answers. Is. each. Or in three Books, price $2 d ., 2 d$., and $3 d$.

Cameron (Mary Lovett). OLD ETRURIA AND MIODERN TUSCANY. With 32 Illustrations. Crozen 8vo. $7 s .6 d$. net.

Cannan (Edwin), M.A. See Sinith (Adam). Canning(George). See Little Library.

Capey (E. F. H.). See Oxford Biographies. Carden (Robert W.). THE CITY OI GENOA. With I2 Illustrations in Colour by Wili.iam Parisinson, and 20 other Illustrations. Demy szo. ros. Gd. net.
Careless (John). See I.P.L.

Carlyle (Thomas). THE FRENCH REVOLUTION. Edited by C. R. L. Fletcher, Fellow of Magdalen College, Oxford. Three Volumes. Cr.8vo. I $8 s$.

THE LETIERS AND SPEECHES OF OLIVER CROMWELL. With an Introduction by C. H. FirTh, M.A., and Notes and Appendices by Mrs. S. C. Lomas. Three Volumes. Demy 8vo. I8s. net.

Carlyle (R. M. and A. J.), M.A. See Leaders of Religion.

Carmichael (Philip). ALL ABOUT PHILIPPINE. With 8 Illustrations. Cr. 8zio. 2s. 6 d.

Carpenter(Margaret Boyd). THECHILD IN ART. With 50 Illustrations. Second Edition. Large Cr. 8vo. 6s.

* Carter (George), MIA. THE STORY OF MILTON'S 'PARADISE LOST.' Crown 8vo. Is. 6 d.

Cavanagh (Francis), M.D. (Edin.). See New Library of Medicine.

Celano(Brother Thomas of). THE LIVES OF FRANCIS OF ASSISI. Translated by A. G. FerRers Howell. With a Frontispiece. Cr. 8vo. 5s. net.

Chambers (A. M.). A CONSTITU. TIONAL HISTORY OF ENGLAND. Crown 8vo. 6s.

Chamisso (A. von). See Simplified German Texts.

Chandler (Arthur), Bishop of Bloemfontein. ARA CQELI: AN ESSAY IN Mrstical Theology. Third Edition. Crown Svo. 3 s. 6 d. net.

Channer (C. C.) and Roberts (M. E.). LACEMIKING IN THE MIDLANDS, PAST AND PRESENT. With I7 fullpage Illustrations. Cr. 8zo. 2s. 6i.

Chapman (S. J.). See Books on Business.

Chatterton (Thomas). See Standard Library:

Chesterfield (Lord), THE LETTERS OF THE EARL OF CHESTERFIELD TO HIS SON. Edited, with an Introduc. tion by C. Strachey, with Notes by A. Calthrup. Truo Volumes. Cr.8ro. izs.

Chesterton(G.K.). CHARLES DICKENS. With two Portraits in Photogravure. Fifth Edition. Cr. 8z'o. $6 s$.

ALL THINGS CONSIDERED. Fourth Edition. Ficas. 8ro. 5 s.

TREMENDOUS TRIFLES. Fcap. 8 $8{ }^{\prime} 0$. $5 s$.

Childe (Charles P.), B.A., F.R.C.S. See New Library of Medicine.

Cicero. See Classical Translations.

Clapham (J. H.), Professor of Economics in the University of Leeds. THE WOOLLEN AND IVORSTED INDUSTRIES. With 2 I Illustrations and Diagrams. $\mathrm{Cr}$. 8zio. 6.s.

Clarke(F.A.), M.A. See Leaders of Religion.

Clausen (Gcorge), A.R.A., R.W.S. SIX LECTURES UN PAINTING. With Io 


\section{General Literature}

Illustrations. Third Edition. Large Post 8v0. 3s. 6\%. net.

AIMS AND IDEALS IN ART. Eight Lectures delivered to the Students of the Royal Academy of Arts. With 32 Illustra. tions. Second Edition. Large Post 8vo. 5s. net.

Clay (Rotha Mary). See Antiquary's Books.

Cleather (A. L.). See Wagner (R).

Clinch (G.), F.G.S. See Antiquary's Books and Little Guides.

Clough (W. T.) and Dunstan (A. E.). See Junior School Books and Textbooks of Science.

Clouston (T. S.), M.D., C.C.D., F.R.S.E. See New Library of Medicine.

Coast (W. G.), B.A. EXAMINATION PAPERS IN VERGIL. Cr. 8zo. 2s.

Cobb (W. F.), M.A. THE BOOK OF PSALMS : with an Introduction and Notes. Denzy 8vo. Ios. 6d. net.

* Cockshott (Winifred), St. Hilda's Hall, Oxford. THE PILGRIM FATHERS, Their Church and Colony. With I2 Illustrations. Demy 8vo. 7s. 6d. net.

Collingwood (W. G.), M.A. THE LIFE OF JOHN RUSKIN. With Portrait. Sixth Edition. Cr. 8vo. 2s. 6d. net.

Collins (W. E.), M.A. See Churchman's Library.

Colvill (Helen H.). ST. TERESA OF SPAIN. With 20 Illustrations. Demy 8vo. 7s. 6d. net.

Combe (William). See I.P.L

Conrad (Joseph). THE MIRROR OF THE SEA: Memories and Impressions. Third Edition. Cr. 8vo. 6 s.

Cook (A. M.), M.A., and Marchant (E. C.) M.A. PASSAGES FOR UNSEEN TRANSIATION. Selected from Latin and Greek Literature. Fourt/ Ed. Cr.8vo. $3 \mathrm{~s} .6 \mathrm{~d}$.

LATIN PASSAGES FOR UNSEEN TRANSLATION. Cr. 8vo. Is. $6 d$.

Cooke $=$ Taylor $(R$. W. $)$. THE FACTORY SYSTEM. Cr. 8vo. 2s. 6a'.

Coolidge (W. A. B.), M.A. THE ALPS With many Illustrations. Demy $8 v 0$. 7s. 6 d. net.

Cooper (C. S.), F.R.H.S. See Westell(W.P.)

Corkran (Alice). See Little Books on Art.
Cotes (Rosemary). DANTE'S GARDEN. With a Frontispiece. Second Edition. Fcap. 8vo. 2s. 6d.; leather, 3s. 6d. net.

BIBLE FLOWERS. With a Frontispiece and Plan. Fcap. 8vo. 2s. 6d. net.

Cotton (Charles). See I.P.L. and Little Library.

Coulton (G. G.). CHAUCER AND HIS E N G L N D. With 32 Illustrations. Second Edition. Demy 8vo. ros. 6d. net. Cowley (Abraham). See Little Library.

Cowper (William). THE POEMS. Edited with an Introduction and Notes by J. C. BAILEY, M.A. Illustrated, including two unpublished designs by WILLIAM Blake. Demy 8vo. ios. $6 d^{\text {. net. }}$
Cox (J. Charles). See Ancient Cities, Antiquary's Books, and Little Guides.

Cox (Harold), B.A., M.P. LAND NATIONALIZATION AND LAND TAXATION. Second Edition revised. Cr. 8vo. 3s. 6d. net.

Crabbe (George). See Little Library.

Craik (Mrs.). See Little Library.

Crane (C. P.), D.S.O. See Little Guides.

Crane (Walter), R.W.S. AN ARTIST'S REMINISCENCES. With I23 Illustrations by the Author and others from Photographs. Second Edition. Demy 8vo. I8s. net.

INDIA IMPRESSIONS. With 84 Illustrations from Sketches by the Author. Second Edition. Demy 8vo. 7s. 6d. net.

Crashaw (Richard). See Little Library.

Crispe (T. E.), K.C. REMINISCENCES OF A K.C. With Portraits. Deme 8vo. Ios. 6 d. net.

Cross (J. A.), M.A. THE FAITH OF THE BIBLE. Fcap. 8vo. 2s. 6d. net.

*Crowley (Ralph H.). THE HYGIENE OF SCHOOL LIFE. Cr. 8vo. 3s. 6 d. net.

Cruikshank(G.). THE LOVING BAL. LAD OF LORD BATEMAN. With II Plates. Cr. I6mo. Is. 6d. net.

Crump (B.). See Wagner (R.).

Cruttwell (C. T.), M.A., Canon of Peterborough. See Handbooks of English Church History.

Cunynghame (H.H.), C. B. See Connoisseur's Library.

Cutts (E. L.), D.D. See Leaders of Religion. Daniell (G. W.), M.A. See Leaders of Religion.

Dante (Alighieri). LA COMMEDIA DI DANTE. The Italian Text edited by Paget Toyniee, M.A., D. Litt. Cr.8vo. 6s.

THE DIVINE COMEDY. Translated by H. F. CARY. Edited with a Life of Dante and Introductory Notes by PAGET Toynbee, M.A., D. Litt. Demy 8vo. 6d.

THE PURGATORIO OF DANTE. Translated into Spenserian Prose by C. GORDON WRIGHT. With the Italian text. Fcap. 8vo. 2s. 6d. net.

See also Little Library, Toynbee (Paget) and Vernon (Hon. W. Warren).

Darley (George). See Little Library.

D'Arcy (R. F.), M.A. A NEW TRIGON OMETRY FOR BEGINNERS. With numerous diagrams. $\mathrm{Cr} .8 v 0.2 s .6 d$.

Daudet (Alplionse). See Simplified French Texts.

Davenport (Cyril). See Connoisseur's Library and Little Books on Art.

Davenport (James). THE WASH. BOURNE FAMILY. With I5 Illustrations and a Map. Royal 8vo. 2 Is. net.

Davey (Richard.) THE PAGEANT OF LONDON. With 40 Illustrations in Colour by John Fulleylove, R.I. InTwo Volumes. Demy 8vo. I 5s. net.

See also Romantic History.

Davies (Gerald S.). See Classics of Art. 
Davies (IV. O. P.). See Junior Fxamination Series.

Davis (H. IV. C.), M.A., Fellow and Tutor of Ialliol Collere. TiNGLANJ) UNJ)lik 'THL' NORMANS ANI) ANGEVINS: I066-I272. With Maps and Illustrations. Demy, 8\%o. IOs. 6d. nict.

Dawson (Nelson). See Connoisseur's Library. Dawson (Mrs. Nelson). See Little Books ors Art.

Deane (A. C.). See Little Library.

Deans (Storry R.). THE TRIALS OF FIVE OUEEN S : KATHARINE OF Aragon, Anne Boleyn, Mary Quee: of Scots, Marie Antoinet t F and Caro LINE OF PRUNSWICK. With I I Ilustrations. Demy 8vo. ros. 6d. wet.

Dearmer (Mabel). A CHILD'S LIFE OF CHRIST. With 8 Illustrations in Colour by E. Fortescue-Brick Dale. Large $\mathrm{Cr}$. 8\%'0. $6 s$.

* D'Este(Margaret). IN THI C $\perp$ NARIFS WITIl A CAMIIIRA. Illustrated. $C$. 8in $7 s$. Gd. net.

Delbos(Leon). THI METRIC SYSTEM Cr. 8210.025

Demosthenes. AGAINST CONON AND CALLICLES. Fdited by F. DARWIN Swirt, M.A. Second Eidition. Forp. Brio. 2s.

Dickens (Charies). See Iittle Library, I.P.L., and Chesterton (G. K.).

Dickinson (Emily). I'OENS. Cr. Szo. 4 s. 6 d. net.

Dickinson (G. L.), MI.A., Fellow of Kinc College, Cambridge. THE GREEL VIEW OF LIFI. Sixth Edition. Cr. 8ro. $2 s .6 \pi$.

Dilke (Lady), Bulley (Miss), and Whitley (Miss). WOMLN'S WORK. Cr. Sio. is. $6 \pi$.

Dillon (Edward), M.A. See Connoisseur's Library, Little Books on Art, and Classics of Art.

Ditchfield (P. H.) M.A. F.S.A. THE STORY OF OUR ENGLISH TOWNS. With an Introduction by Augustus Jessopr, D.D. Second Edition. Cr. Sr:o. Gs.

OLD INGLISH CUSTOMS: Extant at the Present Time. Cr. 8*0. $6 s$.

ENGLISH VIT,AGES. With Ioo Illustra. tions. Second Fitition. Cr. 8ro. as, 6r.net.

THE PARISH CI,ERK. Witl 3 I Illustrations. Third Eatition. Demy 8ro. 7s. 6 . net.

THE OLI)-TIME PARSON. With I7 Illustrations. Second Edition. Denysion. $7 s .6 d^{2}$. net.

Dixon (IV. M.), M.A. A PIRIMIER OF TENNYSON. Trird Edition. Cr. Sro. $25.6 d^{2}$.

ENGLISH POETRI TROM BLAKE TO BROWNING. Seconcl Edition. Cr. Ere. $2 s .6$.

Dobbs (W. J.), M.A. See 'Textbools of Science.

Doney (May). SONGS OF THE REAL. Cr. 8iro. $3^{s .6 \pi}$. net.
Douglas (llugh A.). TINICE ON FOOT. With the Itinerary of the Grand Canal. With 75 Illustrations and II Maps. Frap. 8 wo. 5s. net.

Douglas (James). TIE MAN IN THE PULI'T. Cr: 8\%o, 2s. 6i. net.

Dowden (J.), D.J., Lord Bishop of Edin. burgh. FURTHER STULIES IN THE PRAYEK LOOK. Cr. 2z'o. $6 s$. See also Churchman's Library:

1)rage (G.). See Books on Business.

Uriver (S. R.), D.D., D.C.L., Regius Professor of Hebrew in the University of Oxford. SERMONS ON SUBJECTS CONNECTED IVITH THE OLD TESTAMENT. Cr. 8vo. 6s.

See also Westminster Commentaries.

Dry (Wakeling). See Little Guides.

Dryhurst (A. R.). See Little Books on Art. 'Duff (Nora). MIATII,DA OF TUSCANY. With many Illustrations. Dcmy 8vo. Ios. 6d. net.

Duguid (Charles). See Books on Business. Dumas (Alcxandre). THE CRIMIES OF THE BORGIAS AND O'IHERS. With an Introduction by R. S. GARNETT. With g Illustrations. Cr. Svo. $6 s$

THE CRIMIES OF URIAIN GRAN1)IER AND OTHERS. With 8 Illustrations. Cr. 8ño. $6 s$.

THE CRIMIES OI THE MARQUISE DE BRINVILLIERS AND OTHERS. With 8 Illustrations. Cr. 8 $\pi^{\circ} .6 s$.

THE CRIMES OF ALI PACHA AND OTHERS. With 8 Illustrations. Cr. 8 vo.

MS. MEMIOIRS. Translated by E. M. WALLER. With an Introduction by ANDREIV LANG. With Frontispieces in Photogravure. In six Volumes. Cr. 8vo. 6s. each volume. VOL. I. I802-I82I. VOL. IV. I830.I83I. VOL. II. IS22-I825. VOL. V. I831-I832. VOL. III. I826-I830. VOI. VI. I832-I833.

MI PETS. Newly translated by A. R. ALLiNSON, M.A. With I 6 Illustrations by V. LECONTE. Cr.8\%o. $6 s$.

See also Simplified French Texts.

Duncan(David), D.Sc., LL.D. THE LIFF AND LETTERS OF HERBERT SPENCER. With I Illustrations. Demy Sio. I 15 .

Dann (J.T)., D.Sc. and Mundella (V. A.). GENERAL ILIIIENTARY SCIENCE. With II4 Illustrations. Second Edition. Cr. 8ro. $3 s .6 \%$.

Dunn=Pattison (R. P.). NAPOLEON'S MARSHALS. With zo Illustrations. Demy 8\%0. Sccord fidition. I2s. 6d. net.

Dunstan (A. E.), B.Sc. (Lond.). See Text. books of Science, and Junior School Books.

Durham (The Earl of ). A REPORT ON CANAIA. With an Introductory Note. Denty 8ro. 4s. 6d. net.

Dutt(W. A.). 'THE NORFOLK BROADS. With coloured Illustrations by FrANk Southgate, R.B.A. Second Edition. Cr. s:o. $6 s$. 


\section{General Literature}

WILD LIFE IN EAST ANGLIA. With I 6 Illustrations in colour by Frank SouTH. GATE, R.B.A. Second Edition. Demy 8vo. 7 s. 6 d. net.

SOME LITERARY ASSOCIATIONS OF EAST ANGLIA. With 16 Illustrations in Colour by W. DExTER, R.B.A., and 16 other Illustrations. Deny 8vo. 10s. 6d. net. See also Little Guides.

Earle(John), Bishop of Salisbury. MICRO. COSMOGRAPHIE, OR A PIECE OF THE WORLD DISCOVERED. Post I6mo. 2s. Het.

Edmonds(Major J. E.), R.E.; D.A.Q.-M.G. See IVood (W. Birkbeck).

Edwardes (Tickner). THE LORE OF THE HONEY BEE. With;24 Illustra tions. Cr. 8vo. 6s.

Edwards (Clement), M.P. RAILWAY NATIONALIZATION. Second Edition, Revised. Crowen 8vo. 2s. 6d. net.

Edwards (W. Douglas). See Commercial Series.

Egan (Pierce). See I.P.L.

Egerton (H. E.), M.A. A HISTORY OF BRITISH 'COLONIAL POLICY Second Ed., Revised. Denry 8vo. 7s.6d. net.

Ellaby (C. G.). See Little Guides.

Ellerton (F. G.). See Stone (S. J.).

Epictetus. See Aurelius (Marcus).

Erasmus. A Book called in Latin ENCHIRIDION MILITIS CHRISTIANI, and in English the Manual of the Christian Knight. Ficap. 8vo. 3s. 6d. net.

Erckmann-Chatrian. See SimplifiedFrench Texts.

Evagrius. See Byzantine Texts.

Ewald (Carl). TWO LEGS, AND OTHER STOR1ES. Translated from the Danish by Alexander Teixeira dE Matros. Illustrated by Augusta Guest. Large C $r$. $8 v 0.6 s$.

Ezekiel. See Westminster Commentaries.

Facon (H. T.), B.A. See Junior Examination Series.

Fairbrother(W. H.), M.A. THE PHILO. SOPHY OF T. H. GREEN. Second Edition. Cr. 8vo. 3 s. $6 \mathrm{ct}$.

Fea (Allan). THE FLIGHT OF THE IKING. With over 70 Sketches and Photographs by the Author. New and revised Edition. Demy 8zo. 7s. 6d. net.

SECRET CHAMEERS AND HIDING. PLACES. With 80 Illustrations. Nezu anct revised Edition. Demy 8vo. 7s. 6d. net.

JAMES 11. AND HIS WIVES. With 40 Illustrations. Demy 8vo. 1os. 6d. net.

Fell (E. F. B.). THE FOUNDATIONS OF LIBERTY. Cr. 8vo. 5s. net.

Ferrier (Susan). See Little Library.

Fidler ('T. Claxton), M.Inst. C.E. See Books on Business.

Fielding (Henry). See Standard Library.

Finn (S.W.), M.A. See Junior Examination Series.

Firth (J. B.). See Little Guides.

Firth (C. H.), M.A., Regius Professor of Modern History at Oxford. CROM.
WELL'S ARMY: A History of the English Soldier during the Civil Wars, the Commonwealth, and the Protectorate. Cr. 8vo. 6s.

*Firth (Edith E.). See Beginner's Books and Junior School Books.

FitzGerald (Edward). THE RUBÁIYÁT OF OMAR KHAYYÁM. Printed from the Fifth and last Edition. With a Com. mentary by Mrs. STEPHEN BATson, and a Biography of Omar by E. D. Ross. Cr. 8vo. 6s. See also Miniature Library.

FitzGerald (H. P.). A CONCISE HAND. BOOK OF CLIMBERS, TIVINERS, AND WALL SHRUBS. Illustrated. Fcap. 8vo. 3s. 6d. net.

Fitzpatrick (S. A. O.). See Ancient Cities.

Flecker (W. H.), M.A., D.C.L., Headmaster of the Dean Close School, Cheltenham. THE STUDENT'S PRAYER BOOK. The Text of MIorning and Evening Prayer and Litany. With an Introduction and Notes. Cr. 8vo. 2s. $6 d$.

Fletcher (C. R. L.), M.A. See Carlyle (Thomas).

Fletcher (J. S.). A BOOK OF YORK. SHIRE. With I6 Illustrations in Colour by WaL Paget and Frank Southgate, R.B.A., I6 other Illustrations and a Map. Demy 8vo. 7s. 6\%. net.

Flux (A. W.), M.A., William Dow Professor of Political Economy in M ${ }^{\circ}$ Gill University, Montreal. ECONOMIC PRINCIPLES. Demy 8vo. 7s. 6d. net.

Foat (F. W. G.), D.Litt., M.A. A LONDON READER FOR YOUNG CITI. ZENS. With Plans and Illustrations. $C r$. $8 \% 0$. Is. $6 d$.

Ford (H. G.), MI.A., Assistant Master at Bristol Grammar School. See Junior School Books.

Forel (A.). THE SENSES OF INSECTS. Translated by MACLEOD YEARSLEY. With 2 Illustrations. Demy 8vo. Ios. 6d. net.

Fortescue (Mrs. G.). See Little Boolis on Art.

Fouqué (La Motte). SINTRANI AND HIS COMPANIONS. Translated by A. C. FARQUHARSON. With 20 Illustrations by Edmund J. Sullivan, and a Frontispiece in Photogravure from an engraving by Albrecht DÜRER. Demy 8vo. 7s. 6d. net. Half White Vellum, Ios. $6 \pi$. net.

See also Simplified German Texts.

Fraser (J. F.) ROUND THE WORLI ON A WHEEL. With roo Illustrations. Fifth Edition Cr. 8vo. 6s.

French(W.), M.A. See Textbooks of Science. Freudenreich (Ed, von). DAIRY BACTERIOLOGY. A Short Manual for Students. Translated by J. R. AinsworTH Davis, M.A. Second Edition. Revised. Cr. 8vo. 2s. 6 d.

Fursdon (F. R. $M$ ). FRENCH AND ENGLISH PARALLELS. Fcap. $8 v 0$. 3s. 6 d. net.

Fyvie(John). TRAGEDY OUEENS OF THE GEORGIAN ERA. With 16 Illustrations. Second Ed. Demy8vo. 12s.6\%.net. 
Gallaher (D.) and Stead (W. J.). THE COMPLETE RUGBY FOOTBALLER, ON THE NEW ZEALAND SYSTEM. With 35 Illustrations. Second Ed. Demy S:0. Ios. 6i. niet.

Gallichan (W. MI.). See Little Guides.

Galton(Sir Francis), F.R.S.; D.C.L. Oxf.; Hon. Sc.D., Camb.; Hon. Fellow Trinity Colleze, Cambriçse. MEMORIES OF
MY LIFE. With \& Illustrations. Third Edition. Den'y Ezo. ros. 6d. net.

Gambado (Geofirey, Esq.). See I.P.L.

Garnett (Lucy $M$ I. J.). THE TURKISH PEOPLE: Their Social Life, Religious Beliefs AND INSTITUtions, AND Domestic Life. With 2 I Illustrations. Demy 8vo. ros. 6d. net.

Gaskell (Mrs.). See Little Library, Standard Library and Sixpenny Niovels.

Gasquet, the Right Rev. Abbot, O.S.B. See Antiquary's Books.

Gee (Henry), D.D., F.S.A. See Handbooks of English Church History.

George(H. B.), M.A., Fellow of New College, Oxiord. BATTLES OF ENGLISH HIS. Edition Revise?. Cr. Evo. 35. 6 d.

A HISTORICAL GEOGRAPHY OF THE BRITISH EIIPIRE. Fourth Eaition. Cr. Eะo. $35.6 d$.

Gibbins (H. de B.), Litt.D., M.A. INDUSTRY IN ENGLAND : HISTORI CAL OUTLINES. With 5 Maps. Fifin Edition. Deny Ezio. Ios. 6d.

THE INDUSTRIA HISTORY OF ENGLAND. With Maps and Plans. Fifteenth Editicn, Revised. Cr. 8:0. 35.

ENGLISH SOCIAL REFORMERS Second Edition. Cr. Eiro. 2s. Ed.

See also Hadfield (R. A.)., and Commercial Series.

Gibbon (Edward). MEMOIRS OF MY LIFE AND WRITINGS. Ecited by G BiRKBECK Hill, LL.D Cr. 8:0. 65.

*THE DECLINE AND FALL OF THE ROMAN EMPIRE. Ediied, wih Notes, Appendices, and Mape, by J. B. Biry. M.A., Litt. D., Regius Professor of Modern History at Cambridce. Illustrated. Int Seven Volumes. Demy Evo. Gilt top. Each ros. 6d. net.

Gibbs (Philip). THE ROMANCE OF GEORGE VILLIERS : FIRST DUKE OF BUCKINGHAM, AND SOME MEN AND WONEN OF THE STUART COURT. With 20 Illustrations. Second Edition. Demy 8vo. I5s. net.

Gibson (E. C. S.), D.D., Lord Bishop of Gloucester. See Westminster Commentaries, Handbooks of Theology, and Oxford Biographies.

Gilbert(A. R.). See Little Books on Art.

Gloag (M. R.) and Wyatt (Kate MI.). A BOOK OF ENGLISH GARDENS. With 24 Illustrations in Colour. Demy 8io. ros. 6d. net.
Glover (T. R.), M.A., Fellow and Classical Lecturer of St. John's College, Cambridge. THE CONFLICT OF RLLIGIONS IN Third Edition. Demy 8vo. 7s. 6d. net.

Godfrey (Elizabeth). A BOOK OF RE. MEMBRANCE. Being Lyrical Selections for every day in the Year. Arranged by. Second Edition. Fcat. Ero. 2s 6d. net.

ENGLISH CHILDREN IN THE OLDEN TIME. With 32 Illustrations. Second Edition. Deny Ẽo. 7s. 6d. net.

Godley (A. D.) M.A., Fellow of Magdalen College, Oxford. OXFORD IN THE EIGHTEENTH CENTURY. With r6 Illustrations. Second Edition. Demy 8vo. 7 s. $6 d_{\text {. net. }}$.

Also published in a Colonial Edition.

LYRA FRIVOLA. Fourth Edition. Fcap. 87.0. 2s. 6 d.

VERSES TO ORDER. Second Edition, Fcap. 8\%o. 25. 6 $\%$.

SECOND STRINGS. Fcap. 8z0. 2s. 6d.

Goldsmith (Oliver). See I.P.L. and Standard Library.

Goll (August). CRIMINAL TYPES IN SHAKESPEARE. Authorised Translation from the Danish by Mrs. Charles WEEkEs. Cr. Evo. 55. net.

Gomme (G. L.). See Antiquary's Books.

Gordon (Lina Duff) (Mrs. Aubrey Waterfield). HOME LIFE IN ITALY: LETTERS FROM THE APENNiNes. With I3 Illustrations by AUBREY WATERFIELD and 15 Illustrations from Photographs. Second Edition. Dem" 8vo. Ios. 6d. net.

Gorst (Rt. Hon. Sir John). See New Library of Medicine.

Gostling (Frances M.). THE BRETONS AT HOME. With I2 Illustrations in Colour by Gastox Faxty Lescure, and 32 from Photographs. Demy 8vo. ros. $6 d^{2}$. izet.

Goudge (H. L.), M.A., Principal of Wells Theological College. See Westminster Commentaries.

Graham (Harry). A GROUP OF SCOT. TISH WOMEX. With I6 Illustrations. Second Edition. Demy 8vo. Ios. 6d. net.

Graham (P. Anderson). THE RURAL EXODUS. The Problem of the Village and the Town. Cr. 8zo. 2s. $6 d$.

Grahame (Kenneth). THE WIND IN THE WILLOWS. With a Frontispiece by GrahaM ROBERTSON. Fourth Edition. Cr. 8vio. $6 s$.

Granger (F. S.), M.A., Litt.D. PSYCH. OLOGY. Third Edition. Cr. 8vo. 2s. 6d.

THE SOUL OF A CHRISTIAN. Cr. 8ะ0. 6s.

Gray(E. M'Queen). GERMAN PASSAGES FOR UNSEEN TRANSLATION. $C r$. Sio. 25. $6 d$.

Gray (P. L.), B.Sc. THE PRINCIPLES OF MAGNETISM AND ELECTRICITY. With I8 I Diagrams. Cr. 82'0. 3s. 6 d.

Green (G. Buckland), M.A., late Fellow of St. John's College, Oxon. NOTES ON 
GREEK AND LATIN SYNTAX. Second Ed. revised. Crown 8vo. 3s. 6d.

Green (Mary Anne Everett). ELIZA. BETH; ELECTRESS PALATINE AND QUEEN OF BOHEMIIA. Revised by her Niece S. C. Lomas. With a Prefatory Note by A. W. WARD, Litt.D. Demy 8ro. Ios. 6 d. net.

Greenidge (A.H.J.), M.A., D.Litt. A HISTORY OF ROME : From the Tribunate of Tiberius Gracchus to the end of the Jugurthine War, B.C. I33-104. Demy 8vo. Ios. 6 d. net.

Gregory (Miss E. C.). See Library of Devotion.

Grubb (H. C.). See Textbooks of Technology. Gwynn (Stephen), MI.P. A HOLIDAY IN CONNEMARA. With I6 Illustrations. Denry 8vo. Ios. 6d. net.

Hadfield (R. A.) and Gibbins (H. de B.). A SHORTER WORKING DAY. Cr. 8vo. 25.6 .

*Hall (Cyril). THE YOUNG CARPEN. TER. With Diagrams, and Illustrations. Cr. 8vo. $5 s$.

Hall (Hammond). THE YOUNG EN. GINEER: OR MIODERN ENGINES AND THEIR MOdels. With 85 Illustrations. Second Edition. Cr. 8ro. 55 .

Hall (Mary). A WOMAN'S TREK FROM THE CAPE TO CAIRO. With 64 Illus. trations and 2 Maps. Second Edition. Demy 8vo. I6s. net.

Hamel (Frank). FAMOUS FRENCH SA L O N S. With 20 Illustrations. Third Edition. Demy 8jo. I2s. 6d. net.

Hannay (D.). A SHORT HISTORY OF THE ROYAL NAVY. Vol. I., I2I7-1688. Vol. II., I689-I815. Demy 8चio. Each7s.62. net.

Hannay (James O.), M.A. THE SPIRIT AND ORIGIN OF CHRISTIAN MONASTICISM. Cr.8vo. $6 \mathrm{~s}$.

THE WISDOM OF THEDESERT. Fcap. 8vo. 3s. 6\%. net.

Hardie (Martin). See Connoisseur's Library.

Hare (A. T.), M.A. THE CONSTRUCTION OF LARGE INDUCTION COILS. With 35 Illustrations. Demy 8vo. 6s.

Harker (Alfred), MI.A., F.R.S., Fellow of St. John's College, and Lecturer in Petrology in the University of Cambridge. THE NATURAL HISTORY OF IGNEOUS ROCKS. With II2 Diagrams and 2 Plates. Demy 8vo. I2s. 6d. net.

Harper (Charles G.). THE AUTOCAR ROAD-BOOK. In three Volumes. Crown 8vo. Each 7s. 6d. net.

Vol. I. -SOUTH OF IHE THAMES.

Harvey (Alfred), MI.B. See Ancient Cities and Antiquary's Books.

Hawthorne(Nathaniel). See Little Library.

${ }^{*}$ Headley (F. W.). DARWINISM AND MODERN SOCIALISM. Cr. 800. 5 s. net.

Heath (Frank R.). See Little Guides.
Heath (Dudley). See Connoisseur's Library. Henderson (B. W.), Fellow of Exeter College, Oxford. THE LIFE AND PRINCIPATE OF THE EMPEROR NERO. Illustrated. Nezw and cheaper issue. Demy 8vo. 7s.6\%. net.

AT INTERVALS. Fcap8vo. 2s. 6a. net.

Henderson (M. Sturge). GEORGE MEREDITH : NOVELIST, POET, REFORMER. With a Portrait in Photogravure. Second Edition. Crown 8vo. 6s.

Henderson (T. F.). See Little Library and Oxford Biographies.

Henderson (T. F.), and Watt (Francis). SCOTLAXD OF TO-DAY. With 20 Illustrations in colour and 24 other Illustrations. Second Edition. Cr. 8vo. 6s.

Henley (W. E.). ENGLISH LYRICS. CHAUCER TO POE, I340-1849. Second Edition. Cr. 8\%o. 2s. 6d. net.

Henley (W. E.) and Whibley (C.) A BOOK OF ENGLISH PROSE, CHARACTER, AND INCIDENT, I387-1649. Cr. 8vo. 2s. 6 d. net.

Herbert (George). See Library of Devotion.

Herbert of Cherbury (Lord). See Minia ture Library.

Hett (Walter S.), B.A. A SHORT HIS TORY OF GREECE TO THE DEATH OF ALEXANDER THE GREAT. With 3 Maps and 4 Plans. Cr. 8vo. 3s. $6 d$.

Hewins (W. A. S.), B.A. ENGLISH TRADE AND FINANCE IN THE SEVENTEENTH CENTURY. Cr. $8 v o$. 2s. 6 d.

Hewitt (Ethel M.) A GOLDEN DIAL A Day Book of Prose and Verse. Fcap. 8vo. 2s. 6d. net.

Hey (H.), Inspector, Surrev Education Com mittee, and Rose (G. H.), City and Guilds Woodwork Teacher. A WOODWORK CLASS-BOOK. Pt. I. Illustrated. 4to. $2 s$.

Heywood (IV.). See St. Francis of Assisi.

Hill (Clare). See Textbooks of Technology.

* Hill (George Francis). ONE HUNDRED MASTERPIECES OF SCULPTURE. with Ior Illustrations. Demy 8vo. Ios. $6 d$. net.

Hill (Henry), B.A., Headmaster of the Boy's High School, Worcester, Cape Colony. A SOUTH AFRICAN ARITHMIETIC Cr. 8vo. $3 s .6 d$.

Hind (C. Lewis). DAYS IN CORNWALL. With I6 Illustrations in Colour by WiLlia: PASCOE, and 20 other Illustrations and a Map. Second Edition. Cr. 8vo. 6s.

Hirst (F. W.) See Books on Business.

Hobhouse (L. T.), late Fellow of C.C.C., Oxford. THE THEORY OF KNOWLEDGE. Demy, Svo. Ios. 6a. net.

Hobson(J. A.), M.A. INTERNATIONAL TRADE : A Study of Economic Principles. Cr. 8vo. 2s. 6a. net.

PROBLEMS OF POVERTY. An Inquiry 
into the Industrial Condition of the Poor. Seventh Edition. Cr. 8vo. 2s. 6 .

THE PROBLEM OF THE UNEM. PLOYED. Fourth Edition. Cr.8vo. 2s.6d.

Hodgetts (E. A. Brayley). THE COURT' OF RUSSIA IN THE NINETEENTII CENTURY. With 20 Illustrations. Trwo Volumes. Denty 8vo. 24s. net.

Hodgkin (T.), D.C.L. See Leaders of Religion.

Hodgson(Mrs. W.) HOW TO IDENTIFY OLD CHINESE PORCELAIN. IVith 40 Illustrations. Second Edition. Post8vo. 6s.

Holden-Stone (G. de). See Books on Business.

Holdich (Sir T. II.), K.C.I.E., C.B., F.S.A. THE INDIAN BORDERLAND, $1880 \cdot$ rooo. With 22 Illustrations and a Map. Second Edition. Demy 8vo. ros. 6r. net.

Holdsworth (W. S.), D.C. L. A HISTORY OF ENGLISH LAW. In Four Volumes. Vols. I., II., III. Demy 8vo. Each ros. 6 d. net.

Holland (Clive). TYROL AND ITS PE()PLE. With I6 Illustrations in Colour by ADRIAN STOKES, and 31 other Illustrations. Demy 8z'o. Ios. $6 d^{3}$. net.

Holland (H. Scott), Canon of St. Paul's. See Newman (J. H.).

Hollings (M. A.), M.A. See Six Ages of European History.

Hollway-Calthrop (H. C.), late of Balliol College, Oxford; Bursar of Eton College. PETRARCH : HIS LIFE, WORK, AND TIMES. With 24 Illustrations. Demy 8vo. I $2 s .6$. net.

Holmes (T. Scott). See Ancient Cities.

Holyoake (G.J.). THE CO.OPERATIVE MOVEMENT OF TO-DAY. FourthEd. Cr. 8ro. 2s. 6 d.

Hone (Nathaniel J.). See Antiquary's Books. Hook (A.) H U I A ITY AND ITS PROBLEMS. Cr. $8 \pi 0$. 5s. net.

Hoppner. See Little Galleries.

Horace. See Classical Translations.

Horsburgh (E. L. S.). M.A. LORENZO THE MAGNIFICENT: ANd Florence IN HER GOLDEN AGE. With 24 Illustrations and 2 Maps. Second Eaition. Demy 8vo. 15s. net.

IVATERLOO : With Plans. Second Edition. Cr. 8vo. 55.

See also Oxford Biographies.

Horth (A. C.). See Textbooks of Technology.

Horton(R. F.), 1). D. See Leaders of Religion.

Hosie (Alexander). MANCHURIA. With 30 Illustrations and a Map. Second Edition. Demy 8vo. 7s. 6i. net.

How (F. D.). SIX GREAT SCHOOL MASTERS. With rz lllustrations. Second Edition. Demysro. 7s. 6at.

Howell (A. G. Ferrers). FRANCISCAN DAYS. Being Selections for every diny in the year from ancient Franciscan writings. Cr. 8vo. 3s. 6d. net.

Howell (G.). TRADE UNIONISM-NEW AND OLD. Fourth Edition. Cr. 8vo. $2 s .6 d$.
Huggins (Sir William), K.C.B., O.M. D.C.L., F.R.S. THE ROYAL SOCIETY or, Science in the State and in the Schools. With 25 Illustrations. Wide Royal 8vo. 4s. 6d. net.

Hughes (C. E.). THE PRAISE OF SHAKESPEARE. An English Antho. logy. With a Preface by Sidney LEE. Demy 8vo. 3s. 6i. net.

Hugo (Victor). See Simplified French Texts.

*Hulton (Samuel F.). THE CLERK OF OXFORD IN FICTION. With I2 Illustrations. Demy 8vo. I5s. net.

Hume (Martin), M.A. See Romantic History.

Hutchinson (Horace G.) THE NEW FOREST. Illustrated in colour with 50 Pictures by WALTER TyNDALE and 4 by LuCY KeMp.Welch. Third Edition. Cr. 8vo. 6s.

Hutton (A. W.), M.A. See Leaders of Religion and Library of Devotion.

Hutton (Edward). THE CITIES OF UMBRIA. With 20 Illustrations in Colour by A. P1SA, and 12 other Illustrations. Third Enition. Cr.8:o. 6s.

THE CITIES OF SPAIN. With 24 Illus trations in Colour, by A. W. RIMINGTON, 20 other Illustrations and a Map. Third Edition. Cr. 8vo. 6s.

FLORENCE AND THE CITIES OF NORTHERN TUSCANY, WITH GENOA. With r6 Illustrations in Colour by W11.LIAM PARKINSON, and I6 other Illustrations. Second Eilition. Cr. 8vo. 6s.

ENGLISH LOVE POEMS. Edited with an Introduction. Fcap. 8vo. 3s. 6d. net.

COUNTRY WALKSABOUT FLORENCE. With 32 Drawings by ADELAIDE MARCHI and 20 other Illustrations. Fcap. 8vo. 5s. net.

IN UNKNOWN TUSCANY. With an Appendix by VILliaM HEYwOOd. With 8 Illustrations in Colour and 20 others. Seconc: Edition. Demy 8vo. 7s.6d. net.

* ROMIE. With i6 Illustrations in Colour by MAXWELL ARMFIFLD, and 12 other Illustri. tions. $\mathrm{Cr} .8 \% 0.6 s$.

Hutton (R. H.). See Leaders of Religion.

Hutton (W. H.), M.A. THE LIFE CI SIR THOMAS MIORE. With Portrait after Drawings by Holbein. Seconce Edition. Cr.8\%o. $5 s$.

See also Leaders of Religion.

Hyde (A. G.) GEORGE HEREERT ANI HIS TIMES. With 32 Illustrations. Demy szo. ros. 6d. uet.

Hyett (F. A.). FLORENCE : HFR HISTORY AND ART TO THE FALL OF THE REPUBLIC. Demy Szo. 7s. 6d. net.

Ibsen (Henrik). BRAND. A Drama. Translated by WiLlian WiLson. Third Edition. Cr. 8rio. $3 s .6 \%$.

Inge (W. R.), M.A., Fellow and Tutor of Hertford College, Oxford. CHRISTIAN MYSTICISM. (The Bampton Lectures of r8og.) Demi' 8ro. i2s. 6at. net.

See also Library of Devotion. 


\section{GenERAL Literature}

Innes (A. D.), M.A. A HISTORY OF THE BRITISH IN INDIA. With Maps and Plans. Cr. 8vo. $6 s$.

ENGLAND UNDER THE TUDORS. With Maps. Second Edition. Deny 8vo. ros. 6 d. net.

*Innes (Mary). SCHOOLS OF PAINT. ING. With 76 Illustrations. Cr. $8 v o$.

5s. net. See Churchman's Bible.
Isaiah. Sex

Jackson (C. E.), B.A. See Textbooks of Science.

Jackson (S.), M.A. See Commercial Series.

Jackson (F. Hamilton). See Little Guides.

Jacob (F.), M.A. See Junior Examination Series.

Jeans (J. Stephen). TRUSTS, POOLS, AND CORNERS AS AFFECTING COMMERCE AND INDUSTRY. $C *$. 8vo. 2s. 6 d.

See also Boolss on Business.

Jebb (Camilla). A STAR OF THE SALONS: JULIE DE LESPINASSE. With 20 Illustrations. Demy 8vo. ros. 6d. net.

Jeffery (Reginald W.), M.A. THE HISTORY OF THE THIRTEEN COLONIES OF NORTH AMERICA I497-1763. With 8 Illustrations and a Map. Demy 8vo. 7s. 6d. net.

Jeffreys(D. Gwyn). DOLLY'S THEATRI CALS. Super Royal I6mo. 2s. $6 d$.

Jenks(E.), M.A., B.C.L. AN OUTLINE OF ENGIISḦ LOCAL GOVERNMENT. Second Ed. Revised by R. C. K. ENSOR, M.A. Cr. 8ro. 2s. $6 d$.

Jenner (Mrs. H.). See Little Books on Art.

Jennings (A. C.), M.A. See Handbooks of English Church History.

Jennings (Oscar), M.D. EARLY WOODCUT INITIALS. Denny 4to. 2xs. net.

* Jerningham (Charles Edward). THF MAXIMS OF MARMADUKE. Crown 8vo. 5 s.

Jessopp (Augustus), D.D. See Leaders of Religion.

Jevons (F. B.), M.A., Litt.D., Principal of Hatfield Hall, Durhan. RELIGION IN EVOLUTION. Cr.8vo. 3s.6d. net. See also Churchman's Library and Handbooks of Theology.

Johnson (A. H.), M.A. See Six Ages of European History

Johnston (Sir H. H.), K.C.B. BRITISH CENTRAL AFRICA. With nearly 200 Illustrations and Six Maps. Third Edition. Cr. 4to. I8s. net.

Jones (11.). See Commercial Series.

Jones (H. F.). See Textbooks of Science.

Jones (L. A. Atherley), K.C., M.P., and Bellot (Hugh H. L.), M.A., D.C.L. THE MINER'S GUIDE TO THE COAL MINES REGULATION ACTS AND THE LAW OF EMPLOYERS AND WORKMEN. Cr. 8vo. 2s. 6 . net.

COMMERCE IN WAR. Royalsro. 2Is. net.

Jones (R. Compton), M.A. POEMS OF THE INNER LIFE. Selected by. Thirteenth Edition. Ficap.8vo. 2s.6d. net.
Jonson (Ben). See Standard Library.

Julian (Lady) of Norwich. REVELA. TIONS OF DIVINE LOVE. Ed.by GRACE WARRACK. Third Ed. Cr. 8vo. 3s. 6d.

Juvenal. See Classical Translations.

'Kappa.' LET YOUTH BUT KNOW : A Plea for Reason in Education. Cr. 8vo. 3 s. 6 d. net.

Kaufmann (M.), M.A. SOCIALISM AND MODERN THOUGHT. Second Edition Revised and Enlarged. Cr. 8vo. 2s. 6d. net.

Keats (John). THE POEMS. Edited with Introduction and Notes by E. de SÉlin COURT, M.A. With a Frontispiece in Photogravure. Second Edition Revised. Demy 8vo. 7s. 6r. net.

REALMS OF GOLD. Selections from the Works of. Fcap. 8vo. 3s. 6d. net.

See also Little Library and Standard Library.

Keble (John). THE CHRISTIAN YEAR. With an Introduction and Notes by W. Lock, D.D., Warden of Keble College. Illustrated by R. ANnING Bell. Third Edition. Ficap. 8vo. 3s. $6 d$.; padded morocco, 5 s.

Sce also Library of Devotion.

Kelynack (T. N.), M.D., M.R.C.P. See New Library of Medicine.

Kempis (Thomas a). THE IMITATION OF CHRIST. With an Introduction by Dean Farrar. Illustrated by C. M. Gere. Third Edition. Fcap.8vo. 3s.6d.; padded morocco. 5 s.

Also Translated by C. Bigg, D.D. $\mathrm{Cr}$. 8vo. 3 3. $6 d$.

See also Montmorency (T. E. G. de). Library of Devotion, and Standard Library.

Kennedy (James Houghton), D.D., Assistant Lecturer in Divinity in the University of Dublin. See St. Paul.

Kerr (S. Parnell). GEORGE SELWYN AND THE WITS. With $x 6$ Illustrations. Demy 8vo. xas. 6i. net.

Kimmins (C. W.), M.A. THE CHEMIS TRY OF LIFE AND HEALTH. Illus. trated. Cr. 8vo. 2s. $6 \alpha$.

Kinglake (A. W.). See Little Library.

Kipling (Rudyard). BARRACK-ROOM BALLADS. 9ith Thousand. Trventy sixth Edition. Cr. 8vo. 6s. Also Fipap. 8vo, Leather. $5 s$.

THE SEVEN SEAS. 79th Thousand. Fifteenth Edition. Cr. 8vo. 6s. Also Fcap. 8vo, Leather. $5 s$.

THE FIVE NATIONS. 66th Thousand. Fifth Edition. Cr. 8vo. 6s. Also Fonp. 8vo, Leather. 5 s.

DIPARTMENTAL DITTIES. Seventeenth Edition. Cr. 8vo. 6s. Also Fcap. 8vo, Leather. 5 s.

Knight (Albert E.). THE COMPLETE CRICKETER. With 50 Illustrations. Deny 8vo. 7s. 6d. net.

Knowling (R. J.), M.A., Professor of New Testament Exegesis at King's College, London. See Westminster Commentaries. 
Knox(Winifred F.). THE COURT OF A SAINT. With r6 Illustrations. Demy $8 v 0$. ros. $6 d$. net.

Kropotkin (Prince). THE TERROR IN RUSSIA. Seventh Edition. Cr. 8vo. 2d. net.

Laboulaye (Edouard). See Simplified French Texts.

Lamb (Charles and Mary), THE WORKS. Edited by E. V. LUCAS. Illustrated. I Seven Volumes. Demy8vo. 75.6 t. each.

See also Little Library and Lucas (E. V.)

Lambert (F. A. H.). See Little Guides.

Lambros (Professor S. P.). See Byzantine Texts.

Lane-Poole (Stanley). A HISTORY OF EGYPT IN THE MIDDLE AGES. With Ior Illustrations and a Map. Cr. 8vo. 6s.

Langbridge(F.), M.A. BALLADSOF THE BRAVE: Poems of Chivalry, Enterprise, Courage, and Constancy. Third Edition. Cr. 8vo. 2s. 6d.

Lankester (Sir E. Ray), K.C.B., F.R.S SCIENCE FRONI AN EASY CHAIR. With many rllustrations, of which 2 are in Colour. Cr. 8vo. $6 s$.

Law (William). See Library of Devotion and Standard Library.

Leach (Henry). THE SPIRIT OF THE LINKS. Cr. 8vo. 6s. See also Braid (James)

Le Braz (Anatole). THE LAND OF PARDONS. Translated by FrANCES MI. Gosting. With i 2 Illustrations in Colour by T. C. GoTch, and 40 other Illustrations. Third Edition. Crown 8vo. 6s.

Lees (Beatrice). See Six Ages of European History.

Lees (Frederick). A SUMIMER IN TOURAINE. With I2 Illustrations in Colour by Maxwell, ARMfield, and 8 from Photographs. Also a Map. Seconit Edition. Demy 8ro. ros. 6d. net.

Lelimann(R. C.), M.P. THE COMPLET OARSMAN. With 59 Illustrations. Demy 8vo. Ios. 6i. net.

Lewes (V. B.), M.A. AIR AND WATER Illustrated. Cr. 8ro. 2s. 6d.

Lewis (B. M. Gwyn). A CONCISF HANDBOOK OF GARDEN SHRUBS. With 20 Illustrations. Fcap.8wo. $3^{\text {s. } 6 \text { d. net. }}$

Lindsay (Lady Mabel). ANNI DOMIINI A Gospel Study. In Tivo Tolumes. Super Royal 8vo. ros. net.

Lindsay (W. M.), Fellow of Jesus College, Oxford. See Plantus.

Lisle (FortunCede). See Little Books on Art

Littlehales (H.). See Antiquary's Books.

Llewellyn (Owen) and Raven=Hill (L.). THE SOUTH-BOUND CAR. With 85 Illustrations. Crozun 8vo. 6s.

Lock (Walter), D.D., Warden of Keble College. ST. PAUL, THE MISTERBUILDER. Second Ed. Cr. 8ro. $3 s .6 d$. THE BIBLE AND CHRISTIAN LIFE. Cr. 8vo. 6s. See also Keble (J.) and Leaders of Religion.
Locker (F.). See Little Library.

Lodge (Sir Oliver), F.R.S. THE SUB. SIANCE OF FAITH, ALLIED WITH SCIENCE: A Catechism for Parents and Teachers. Ninth Ed. Cr. 8zo. as net.

MAN AND THE UNIVERSE: A STUDY OF THE INFLUENCE OF THE ADVANCE IN SCIENTIFIC KNOWLEDGE UPON OUR UNDERSTANDING OF CHRISTIANITY. Sixth Edition. Demy 8zo. 7s. 6d. net.

* THE SURVIVAL OF MIAN : A Study of Unrecognised Human Faculty. Demy 8vo. 7s. 6d. net.

Lodge (Eleanor C.). See Six Ages of European History.

Lofthouse (W. F.), M.A. ETHICS AND ATONEMENT. With a Frontispiece. Demy 8vo. 5s. net.

Longfellow (H. W.). See Little Library.

Lorimer (George Horace). LETTERS FROMT A SELF-MADE MERCHANT TO HISSON. Seventeenth Edition. Cr. 8vo. $3 s .6 d$.

OLD GORGON GRAHAMI. Second Edition. Cr. 8vo. 6s.

${ }^{*}$ Lorimer (Norma). BY THE WATERS OF EGYPT. With 12 Illustrations in Colour by BENTON FletCher, and other Illustrations. Demy 8zo. I6s, net.

Lover (Samuel). See I.P.L.

Lucas (E. V.). THELIFE OF CHARLES LAMB. With 28 Illustrations. Fourt/2 and Revised Edition in One Volume. Demy 8vo. 7s. 6d. net.

A WANDERER IN HOLLAND. Witb 20 Illustrations in Colour by HERBERT MIARSHALL, 34 Illustrations after old Dutch Masters, and a Map. Ninth Edition. Cr. 8zo. 6s.

A WANDERER IN LONDON. With I6 Illustrations in Colour by NELson Dawson, 36 other Illustrations and a Map. Scvent/ Edition. Cr. 8rio. 6s.

A WANDERER IN PARIS. With I6 Illus. trations in Colour by IVALTER DeATER, and 32 from Photographs after Old MIasters. Second Edition. Cr. 8ro. 6s.

THE OPEN ROAD : a Little look for Wayfarers. Fifteenth Edition. Fcap. 8vo. 5s.; India Paper, 7s. 6 d.

THE FRIENDLY TOWN : a Little Book for the Urbane. Fourth Edition. Fcap. 8*o. 5s.: India Paper, 7s. 6d.

FIRESIDE AND SUNSHINE. Fourt/z Edition. Fcat. Silo. 5 s.

CHARACTER AND COMIEDY. Fourth Edition. Fach. 8i'o. 5 s.

THE GENTLEST ART. A Choice of Letters by Entertaining Hancis. Fifth Edition. Fcap. 8\%o. 5s.

A SWAN AND HER FRIENDS. With 24 Illustrations. Demy 8च̃o. I2s. 6.t. net.

HER INFINITE VARIETY : A FEMININE Portrait Gallery. Fourth Edition. Ficap. 8vo. 55 .

LISTENER'S LURE: AN OrLIOUE NARRATION. Fifth Edition. Fcap. 8ro. $5 s$. 


\section{GENERAL Literature}

*GOOD COMPANY : A Rally of MEN. Fcap. 8v0. 5s.

ONE DAY AND ANOTHER: A VOLUME OF EsSAYS. Fcap. 800. 5 s.

OVER BEMERTON'S: AN EASY-GOING Chronicle. Sixth Edition. Fcap. 8vo. 5s. net.

See also Lamb (Charles)

Lucian. See Classical Translations.

Lyde (L. W.), M.A. See Commercial Series.

Lydon (Noel S.). A PRELIMINARY GEOMETRY. With numerous Diagrams. Cr. 8vo. Is.

See also Junior School Books.

Lyttelton (Hon. Mrs. A.). WOMEN AND THEIR WORK. Cr. 8vo. 2s. $6 d$.

M. (R.). THE THOUGHTS OF LUCIA HALIDAY. With some of her Letters. Edited by R. M. Fcap. 8vo. 2s.6d. net.

Macaulay (Lord). CRITICAL AND HISTORICAL ESSAYS. Edited by F. C. MoN TAGUE, MI.A. Three Volumes. Cr. 8v0. I8s.

M'Allen (J. E. B.), M.A. See Commercial Series.

McCabe (Joseph) (formerly Very Rev. F. AnTONY, O.S.F.). THE DECAY OF THE CHURCH OF ROME. Demy 8vo. 7s. $6 d$. net.

MacCunn (Florence A.). M A R Y STUART. With 44 Illustrations, in cluding a Frontispiece in Photogravure. New and Cheaper Edition. Large Cr. 8vo. $6 s$.

See also Leaders of Religion.

McDermott (E.R.). See Books on Business.

McDougall (William), M.A. (Oxon., M.B. (Cantab.). AN INTRODUCTION TO SOCIAL PSYCHOLOGY. Cr.8vo. 5s.net.

M'Dowall (A. S.). See Oxford Biographies. MacFie (Ronald C.), M.A., M.B. See New Library of Medicine.

Mackay (A. M.), B.A. See Churchman's Library.

Mackenzie (W. Leslie), M.A., M.D. D.P.H., etc. THE HEALTH OF THE SCHOOL CHILD. Cr. 8vo. 2s. $6 d$.

Macklin (Herbert W.), M.A. See Antiquary's Books.

M'Neile (A. H.), B.D. See Westminster Commentaries.

'Mdlle Mori' (Author of). ST. CATHERINE OF SIENA AND HER TIMES. With 28 Illustrations. Second Edition. Demy 8vo. 7s. 6d. net.

Maeterlinck (Maurice). THE BLUE BIRD: A FAIRY Play in Five ACts. Translated by Alexander TEIXERA DE MatTos. Second Edition. Fcap. 8wo. Deckle Edoes. 3s. 6a. net.

Magnus (Laurie), M.A. A PRIMER OF WORDSWURT'H. Cr. 800. 2s. $6 d$.

Mahaffy (J.P.), Litt.D. A HISTORY OF THE EGYPT OF THE PTOLEMIES. With 79 Illustrations. $C r .8 v 0.6 s$.

Maitland (F. W.), M.A., LL.D. ROMAN CANON LAW IN THE CHURCH OF ENGLAND. Royal8vo. 7s. 6d.

Major(H.), B.A., B.Sc. A HEALTH AND
TEMPERANCE READER. Cr. 8 . IS.

Malden (H. E.), M.A. ENGLISH RE. CORDS. A Companion to the History of England. $C r .8 v 0.35 .6 d$.

THE RIGHTS AND DUTIES OF A CITIZEN. Seventh Edition. Cr. 8vo. Is. 6 d.

See also School Histories.

Marchant (E. C.), M.A., Fellow of Peter. house, Cambridge. A GREEK ANTHO. LOGY Second Edition. Cr. 8vo. 3s. 6d. See also Cook (A. M.).

Marett (R. R.), M. A., Fellow and Tutor of Exeter College, Oxford. THE THRESHOLD OF RELIGION. Cr. 8vo. 3s. $6 d$ net.

Marks (Jeannette), M.A. ENGLISH PASTORAL DRAMA from the Restoration to the date of the publication of the 'Lyrical Ballads' (x660-I798). Cr. $8 v 0$. 5s. net.

Marr (J. E. ), F.R.S., Fellow of St John's Col lege, Cambridge. THE SCIENTIFIC STUDY OF SCENERY. Third Edition. Revised. Illustrated. Cr. 8\%0. 6s.

AGRICULTURAL GEOLOGY. Illustrated. Cr. 8vo. $6 s$

Marriott (Charles). A SPANISH HOLIDAY. With 8 Illustrations by A. M. FowEraker, R.B.A., and 22 other Illustrations. Demy 8vo. 7s. 6d. net.

Marriott (J.A. R.), M.A. THE LIFE AND TIMES OF' LORD FALKLAND. With 23 Illustrations. Second Edition. Demy 8v0. 75. 6d. net.

See also Six Ages of European History.

Marvell (Andrew). See Little Library.

Masefield (John). SEA LIFE IN NEL. SON'S TIME. With r6 Illustrations. $C r$. 8vo. 35. 6el. net.

ON THE SPANISH MAIN: or, SOME ENGLISH FORAYS IN THE ISTHMUS OF DARIEN. With 22 Illustrations and a Map. Demy 8vo. Ios. 6h. net.

A SAILOR'S GARLAND. Selected and Edited by. Second Ed. Cr. 8vo. 3s.6d.net.

AN ENGLISH PROSE MISCELLANY. Selected and Edited by. Cr. 8vo. 6 s.

Maskell (A.). See Connoisseur's Library.

Mason (A.J.), D.D. See Leaders of Religion. Masterman (C. F. G.), M.A., M.P. TENNYSON AS A RELIGIOUS TEACHER. Cr. 8vo. 6s.

THE CUNDITION OF ENGLAND. Second Edition. Cr. 8vo. $6 s$.

Masterman (J. H. B.), M.A. See Six Ages of European History.

Matheson (E. F.). COUNSELS OF LIFE. Fcap. 8vo. 2s. 6d. net.

Maude (J. H.), M.A. See Handbooks of English Church History.

May (Phil). THE PHIL MAY ALBUM. Second Edition. 4to. Is. net.

Mayne (Ethel Colburn). ENCHANTERS OF MEN. With 24 Illustrations. Demy 8vo. ros. 6 d. net. 
Meakin (Annette M. B.), Fellow of the Anthropological Institute. WOMAN IN TRANSITION. Cr.820. $6 s$.

GALICIA: The Switzerland of Spain. With 105 Illustrations and a Map. Demy 8vo. I2s. 6\%. 7zet.

* Medley (D. J.), M.A., Professor of History in the University of Glasgow. ORIGINAL ILLUSTRATIONSOF ENGLISH CON STITUTIONAL HISTORY, COMPRISING a Selected Number of the Chief Charters and Statutes, Cr.8vo. $75.6 d$. net.

Mellows (Emma S.). A SHORT STORY OF ENGLISH LITERATURE. $C r$. $8 v 0.35 .6 d$.

Mérimée (P.). See Simplified French Texts. Methuen (A. M. S.), M.A. TH H TRAGEDY OF SOUTH AFRICA. Cr.8vo. 2s. net. Also Cr. 8vo. 3d. net. ENGLAND'S RUIN : DISCUSSED IN FOURTEEN LetTERS TO A Protectionist. Ninth Edition. Cr. 8r'o. 3r. net.

Meynell (Everard). COROI AND HIS FRIENDS. With 28 Illustrations. Demy $8 v 0$ ros. 6 d. net.

Miles (Eustace), M.A. LIFE AFTER LIFE: OR, The TheORY OF REINCARnATION. Cr. 8vo. 2s. 6d.net.

THE POWER OF CONCENTRATION : How to ACQUIRE IT. Third Edition. Cr. 8vo. 3s. 6d. net.

Millais (J. G.). THE LIFE AND LETTERS OF SIR JOHN EVERE'TT MILLAIS, President of the Royal Academy. With many Illustrations, of which 2 are in Photogravure. New Edition. Demy 8vo. 7s. 6d. net. See also Little Galleries.

Millin (G. F.). PICTORIAL GARDEN. ING. With 2x Illustrations. Croiun $8 v 0$. 3s. 6d. net.

Millis (C. T.), M.I.M.E. See Textbooks of Technology.

Milne (J. G.), M.A. A HISTORY OF EGYPT UNDER ROMAN RULE. With I 43 Illustrations. Cr.8vo. $6 s$.

Milton (John). A DAY BOOK OF MILTON. Edited by R. F. TOWNDROW. Fcap. 8v0. 2s. 6d. net.

See also Little Library and Standard Library.

Minchin (H. C.), M.A. See Peel (R.).

Mitchell(P. Chalmers), M.A. OUTLINES OF BIOLOGY. With 74 Illustrations. Seeond Edition. Cr.8vo. 6s.

Mitton (G. E.). JANE AUSTEN AND HER TIMES. With 2 I Illustrations. Second and Cheaper Edition. Large Cr. svo. 6s.

Moffat (Mary M.). QUEEN LOUISA OF PRUSSIA. With zollustrations. Fourth Edition. Crown 8vo. 6s.

Moil (A.). See Books on Business.

Moir (D. M.). See Little Library.

Molinos (Dr. Michael de). See Library of Devotion.

Money (L. G. Chiozza), M.P. RICHES
AND POVERTY. Eighth Edition Demy 8vo. 5s. net. Also Cr. 8zio. Is. net.

Montagu (Henry), Earl of Manchester. See Library of Devotion.

Montaigne. A DAY BOOK OF. Edited by C. F. POND. Fiap. 8vo. 2s. 6r. net.

Montgomery (H. B.) THE EMPIRE OF THE EAST. With a Frontispiece in Colour and 18 other Illustrations. Second Edition. Demy 8vo. 7s. 6d. net.

Montmorency (J. E. G. de), B.A., LL.S. THOMAS A KEMPIS, HIS AGE AND BOOK. With 22 Illustrations. Second Edition. Demy 8vo. 7s.6\%.net.

Moore (H. E.). BACK IO THE LAND. Cr. 8vo. 2s. 6 .

* Moore (T. Sturge). ART AND LIFE. Illustrated Cr.80io. 5s. net.

Moorhouse (E. Hallam). NELSON'S LADY HAMILTON. With 5I Portraits. Second Edition. Demy 8vo. 7s. 6d. net.

Moran (Clarence G.). See Books on Business. More (Sir Thomas). See Standard Library. Morfill (W. R.), Uriel College, Oxford. A HISTORY OF RUSSIA FROM PETER THE GREAT TO ALEXANDER II. With I2 Maps and Plans. Cr. 8\%o. 3s.6d. Morich (R. J.). See School Examination Series.

Morley (Margaret W.), Founded on. THE BEE PEOPLE. With 74 Illustrations. Sq. Crozun 8vo. 2s. 6 \%.

LITTLE MITCHELL: The STORy OF A Mountain Squirrel told by Himself. With 20 Illustrations. Sf. Cr. 8vo. 2s. 6 d.

Morris (J.). THE MAKERS OF JAPAN. With 24 Illustrations. Demy 8vo. I2s. $6 d$. net.

Morris (Joseph E.). See Little Guides.

Morton (A. Anderson). See Brodrick(M.).

Moule(H. C. G.), D.D., Lord Bishop of Durlam. See Leaders of Religion.

Muir (M. M. Pattison), M.A. THE CHEMISTRY OF FIRE. Illustrated. Cr. 8ro. 2s. 6 d

Mundella (V. A.), M.A. See Dunn (J. T.).

Munro(R.), M.A., LL.D. See Antiquary's Books.

Musset (Alfred de). See Simplified French Text.

Myers (A. Wallis), THE COMPLETE LAWN TENNIS PLAYER. With 90 Illustrations. Second Edition. Demy 8vo. Ios. 6d. net.

Naval Officer (A). See I. P. L.

Newman (Ernest). See New Library of MIusic.

Newman (George), M.D., D.P.H., F.R.S.E. See New Library of Medicine.

Newman (J. H.) and others. See Library of Devotion.

Newsholme (Arthur), M.D., F.R.C.P. See New Library of Medicine.

Nichols (Bowyer). See Little Library.

Nicklin (T.), M.A. EXAMINATION PAPERS IN THUCYDIDES. Cr. 800. 2s. Nimrod. See I. P. L.

Norgate (G. Le Grys). THE LIFE OF 


\section{General Literature}

SIR WALTER SCOTT. With 53 Illustra. tions by JeNnyWYL1E. Demy 8vo.75.6d. net. Norway (A. H.). NAPLES. PAST AND PRESENT. With 25 Coloured Illustrations by Maurice Groiffenhagen. Third Edition. Cr. 810.6s.

Novalis。 THE DISCIPLES AT SAİS AND OTHER FraGments. Euited by Miss Una Birch. Ficap. 8vo. 3s. 6d. net.

Officer (An). See I. P. L.

Oldfield (W. J.), MI.A., Prebendary of Lincoln. A PRIMER OF RELIGION. Based ON THE CATECHISA OF THE CHURC11 of England. Crozun 8vo. 2s. $6 d$.

Oldham (F. M.), B.A. See Textbooks of Science.

Oliphant (Mrs.). See Leaders of Religion.

Oliver, Thomas, M. D. See New Library of Medicine.

Oman(C. W. C.), M.A., Fellow of All Souls' Oxford. A HISTORY OF THE ART OF WAR IN THE MIDDLE AGES. Illustrated. Demy 8vo. Ios. 6d. net.

ENGLAND BEFORE THE CONQUEST. With Maps. Demy 8vo. ios. 6d. net.

Oppé (A. P.). See Classics of Art.

Ottley (R. L.), D.D. See Handbooks of Theology and Leaders of Religion.

Overton (J. H.). See Leaders of Religion.

Owen (Douglas). See Books on Business.

Oxford (M. N.), of Guy's Hospital. A HAND. BOOK OF NURSING. Fifth Edition. Cr. 8vo. 3s. 6 \%.

Pakes (W. C. C.). THE SCIENCE OF HYGIENE. Illustrated. Demy 8vo. Iss.

Parker (Eric). THE BOOK OF THE ZOO; BY DAY AND NIGHT. With 24 Illustrations from Photographs by HENRY IRviNG. Cr. 8vo. 6s.

Parker (Gilbert), M.P. A L O E R'S DIARY. Fcap. 8ro. $5 s$.

Parkes (A. K.) SMALL LESSONS ON GREAT TRUTHS. Fat. 8vo. Is. $6 \pi$.

Parkinson (John). PARADISI IN SOLE PARADISUS TERRESTRIS, OR A GARDEN OF ALL SORTS OF PLEASANT FLOWERS. Folio. $£_{3}$, 3s. net.

Parsons (Mrs. C.). GARRICK AND HIS CIRCLE. With 36 Illustrations. Second Edition. Demy 8vo. 12s. 6d. net.

THE INCOMPARABLE SIDDONS. With 2o Illustrations, Demy 8vo. I2s. 6d. net.

Pascal. See Library of Devotion.

Paston (George). SOCIAL CARICATURE IN THE EIGHTEENTH CENTURY. With 214 Illustrations. Imperial Quarto. f2, I2s. 6d. net.

LADY MARY WORTLEY MONTAGU AND HER TIMES With 24 Illustra. tions. Second Edition. Demy 8vo. 15 s. net.

See also Little Books on Art and I.P. L.

Patmore (K. A.). THE COURT OF LOUIS XIII. With 6 Illustrations. Demry 8vo. Ios. 6 . net.

Patterson (A. H.). NOTES OF AN EAST COAST NATURALIST. Illustrated in Colour by F. Southate, R.B.A. Second Edition. Cr. 8vo. 6s.
NATURE IN EASTERN NORFOLK. With I2 Illustrations in Colour by FRANk Southgate, R.B.A. Second Edition. Cr. Evo. $6 s$.

WILD LIFE ON A NORFOLK ESTU. ARY. With 40 Illustrations by the Author, and a Prefatory Note by Her Grace the DUCHESS OF BeDFord. Demy 8vo. Ios. 6 d. net.

* MAN AND NATURE ON TIDAL WATERS. With Illustrations by the Author. Cr. 8vo. 6s.

Peacock (Netta). See Little Books on Art.

Peake (C. M. A.), F.R.H.S. A CON CISE HANDBOOK OF GARDEN ANNUAL AND BIENNIAL PLANTS. With 24 Illustrations. Ficap. 8vo. 3s.6 $\mathrm{d}_{\text {. net. }}$

Peel (Robert), and Minchin (H. C.), M.A OXFORD. With roo Illustrations in Colour. Cr. 8vo. 6s.

Peel (Sidney), late Fellow of Trinity College, Oxford, and Secretary to the Roval Com mission on the Licensing Laws. PRACTI. CAL LICENSING REFORM. Second Edition. Cr. 8ro. is. 6त.

Pentin (Herbert), M.A. See Library of Devotion.

Petrie(W.M.Flinders), D.C. L., L.L.D., Professor of Egyptology at University College. A HISTORY OF EGYPT: Fully lllustrated. In sixvolumes. Cr. 8vo. 6s. each. VOL. I. FROM THE EARLIFST KINGS TO XVITH DYNaSTY. Sixth Edition.

VOL. II. THE XVIITH AND XVIIITH DyNASTIEs. Fourth Edition.

Voi. III. XIXTH TO XXXTh DyNasties.

VOL. IV. EgY'T UNDER THE PTOLEMAIC Dynasty. J. P. MAHAFFy, Lit. D.

Vol. v. Egypt under Romian Rule. J. G. Milne, M.A.

Vol. VI. EgYPT IN THE Middle Ages, Stanley Lane-Poole, M.A.

RELIGION AND CONSCIENCE IN ANCIENT EGYPT. Lectures delivered at University College, London. Illustrated. Cr. 8vo. 2s. 6d.

SYRIA AND EGYPT, FROM THE TELL EL AMARNA LETTERS. Cr. 8\%o. $2 s .6 d$. EGYPTIAN TALES. Translated from the Papyri. First Series, iv th to X11th Dynasty. Edited by W. M. Finders Petrie. Illus. trated by Tristram Ellis. Second Edition. Cr. 8\%, $35.6 \pi$.

EGYPTIAN TALES. Translated from the Papyri. Second Series. Xvilth to Xixth Dynasty. Illustrated by Tristram Ellis. Crown 8vo. 3s. 6 d.

EGYPTIAN DECORATIVE ART. A Course of Lectures delivered at the Royal Institution. Illustrated. $C r .8 v 0.35 .6 d$.

Phillips (W. A.). See Oxford Hiographies.

Phillpotts (Eden). MY DEVON YEAR. With $3^{8}$ Illustrations by J. Ley PETHY BRIDGE. Second and Cheaper Edition. Laroe Cr. 8vo. 6s.

UP - ALONG AND DOWN - ALONG. Illustrated by CLAUDE SHEPPERSON. Cr. 4 to. 5s. net. 
Phythian (J. Ernest). TREES IN NATURE, MYTH, AND ART. With 24 Illustrations. Cronen 8vo. $6 s$.

Piarr (Victor G.). M.A. See School His. tories.

Plato. See Standard Library.

Plautus. THE CAPTIVI. Edited, with an Introduction, Textual Notes, and a Commentary, by W. M. LindsAY, Fellow of Jesus College, Oxford. Demy 8vo. Ios.6d.net.

Plowden $=$ Wardlaw (J. T.), B.A. See School Examination Series.

Podmore (Frank). MODERN SPIRITUALISM. Tzuo Volumes. Demy 8vo. zIs. net.

MESMERISM AND CHRISTIAN SCIENCE: A Short History of Mental Healing. Demy 8vo. ros. 6r. net.

Pollard (Alice). See Little Books on Art.

Pollard (Alfred W.). THE SHAKESPEARE'S FOLIOS AND QUARTOS. With numerous Facsimiles. Folio. One Guinea net.

Pollard(Eliza F.). See Little Books on Art. Pollock (David), M.I.N.A. See Books on Business.

Potter (M. C.), M.A., F.L.S. A N ELEMENTARY TEXT-BOOK OF AGRICULTURAL BOTANY. Illustrated. Third Edition. Cr. 8vo. $4 s .6 d$.

Power (J. O'Connor). THE MAKING OF AN ORATOR. Cr.8vo. 6s.

Price (Eleanor C.). A PRINCESS OF THE OLD WORLD. With 21 Illus. trations. Demy 8vo. I2s. 6d. net.

Price (L. L.), M.A., Fellow of Oriel College, Oxon. A HISTORY OF ENGLISH POLITICAL ECONOMY FROM ADAM SMITH TO ARNOLD TOYNBEE. Fifth Edition. Cr. 8vo. 2s. 6 d.

Protheroe (Ernest). THE DOMINION OF MAN. GEOGRAPHY IN ITS HUMAN Aspect. With 32 full-page Illustrations. Second Edition. Cr. 8vo. $2 s$.

Psellus. See Byzantine Texts.

Pullen-Burry (B.). IN A GERMAN COLONY; or, FOUR WEEKS IN NEW Britain. With 8 Illustrations and 2 Maps. Cr. 8vo. 5s. net.

Pycraft (W. P.). BIRD LIFE. With Illustrations in Colour by G. E. LOvGE, and others from Drawings and Photographs. Demy 8ro. ros. 6d. net.

' $Q$ ' (A. T. Quiller Couch). THE GOLDEN POMP. A PROCESSION OP ENGLish LyRics FRoM SURREY TO SHIRLEY. Second and Cheaper Edition. Cr. 8vo. $2 s .6$. net.

G. R. and E. S. MR. WOODHOUSE'S CORRESPONDENCE. Cr. 8vo. $6 \mathrm{~s}$.

Also published in a Colonial Edition.

Rackham (R. B.), M.A. See Westminster Commentaries

Rage (Laura M.). THE WOMEN ART ISTS OF BOLOGNA. With 20 Illustrations. Demy 8vo. 7s. 6d. net.

Ragg (Lonsdale). B.D., Oxon. DANTE
AND HIS ITALY. With 32 Illustrations. Demy 8vo. 12s. 6d. net.

Rahtz (F. J.), M.A., B.Sc. HIGHER ENGLISH. Fourth Edition. Cr. 8vo. $35.6 d$.

JUNIOR ENGLISH. Second Edition. Cr. 8710 . is. $6 d$.

Randolph (B. W.), D.D. See Library of Devotion.

Rannie (D. W.), M.A. A STUDENT'S HISTORY OF SCOTIAND. Cr.8vo. 3s. $6 \pi^{\circ}$. WORDSWORTH AND HIS CIRCLE. With 20 Illustrations. Demy 8vo. I2s. $6 d$. net.

Raslidall (Hastings), M.A., Fellow and Tutor of New College, Oxford. DOC TRINE AND DEVELOPMENT. $C r$. 8vo. 6s.

Raven (J.J.), D.D., F.S.A. See Antiquary's Books.

Raven=Hill (L.). See Llewellyn (Owen).

Rawlings (Gertrude Burford). COINS AND HOW TO KNOW THEM. With 206 Illustrations. Second Edition. Cr.8vo. $6 s$.

Rawstorne (Lawrence, Esq.). See I.P.L.

Raymond (Walter). See School Histories.

Rea (Lilian). THE LIFE AND TIMES OF MARIE MADELEINE COUNTESS OF LA FAYETTE. With 20 Illustrations. Demy 8vo. ros. 6d. net.

Read (C.Stanford), M. B. (Lond.), M.R.C.S., L.R.C.P. FADS AND FEEDING. Cr. 8\%0. 2s. $6 \pi$.

Real Paddy (A). See I.P.L

Reason (W.), M.A. UNIVERSITY AND SOCIAL SETTLEMENTS. Edited by Cr. 8vo. 2s. 6d.

Redpath (H. A.), M.A., D.Litt. See Westminster Commentaries.

Rees (J. D.), C.I.E., M.P. THE REAL INDIA. Second Edition. Demy8vo. Ios. 6d. net.

Reich (Emil), Doctor Juris. WO MA N THROUGH THE AGES. With 36 Illus. trations. Trwo Volumes. Demy8vo. 2 Is. net.

Reynolds (Sir Joshua). See Little Galleries.

Rhodes (W. E.). See School Histories.

Ricketts (Charles). See Classics of Art.

Richardson (Charles). THE COMPLETE FOXHUNTER. With 46 Illustrations, of which 4 are in Colour. Second Edition. Demy 8vo. 12s. 6d. net.

Richmond (Wilfrid), Chaplain of Lincoln's Inn. THE CREED IN THE EPISTLES. Cr. 8vo. 2s. 6\%. net.

Riehl(W. H.). See Simplified German Texts. Roberts (M. E.). See Channer (C. C.).

Robertson (A.), D.D., Lord Bishop of Exeter. REGNUM DEI. (The Bampton Lectures of IgoI). A New and Cheaper Edition. Demy 8vo. 7s. 6d. net.

Robertson (C. Grant). M.A., Fellow of All Souls' College, Oxford. SELECT STATUTES, CASES, AND CONSTITUTIONAL DOCUMENTS, 1660.1832. Demy 8vo. ros. 6d. net. 


\section{General Literature}

Robertson (C. Grant) and Bartholomew (J. G.), F.R.S.E., F.R.G.S. A HIS TORICAL AND MODERN ATLAS OF THE BRITISH EMPIRE. Demy Quarto. 4s. 6 d. net.

Robertson(SirG.S.), K.C.S.I. CHITRAL: THE STORY of A MiNOR Siege. With 8 Illustrations. Third Edition. Demy 8vo. ros. $6 d$. net.

Robinson (Cecilia). THE MINISTRY OF DEACONESSES. With an Introduction by the late Archbishop of Canterbury. Cr. 8\%o. 3s. $6 d^{\prime}$.

Robinson (F. S.). See Connoisseur's Library.

Rochefoucauld (La). See Little Library.

Rodwell (G.), B.A. NEW TESTAMENT GREEK. A Course for Beginners. Wit a Preface by WALTER Lock, D.D., Warden of Keble College. Fcap. 8vo. 3s. $6 d$.

Roe (Fred). OLD OAK FURNITURE. With many Illustrations by the Author, includin? a frontispiece in colour. Second Edition. Demy 8vo. ros. 6d. net.

Rogers (A. G. L.), M.A. See Books on Business.

Roland. See Simplified French Texts.

Romney (George). See Little Galleries.

Roscoe (E. S.). See Little Guides.

Rose (Edward). THE ROSE READER. Illustrated. Cr. 8vo. 2s. 6d. Also in Parts. Parts I. and II. 6d. each; Part III. 8d.; Part IV. Ior

Rose (G. H.). See Hey (H.) and Baring Gould (S)

Rowntree (Joshua). THE IMPERIAI DRUG TRADE. A RE-STATEMENT OI THE OpIUM OUESTION. Third Edition Revised. Cr. 8vo. 2s. net.

Royde=Smith (N. G.). THE PILLOW BOOK: A GARNER OF MANY MoODS. Collected by. Second Edition. Cr. $8 v 0$. 4s.6d. net.

POFTS OF OUR DAY. Selected, with an Introduction, by. Fcap. 8vo. 5 s.

Rubie (A. E.), D.D. See Junior School Books.

Rumbold (The Right Hon. Sir Horace). Bart., G.C. B. G.C. M. G. TH AUSTRIAN COURT IN THE NINE TEENTH CENTURY. With t6 Illustrations. Derny 8vo. I8s. net.

Russell (Archibald G. B.). See Blake (William.)

Russell (W. Clark). THE LIFE OF ADMIRAL LORD COLLINGWOOD. With I2 $_{2}$ Illustrations by F. BRANGWYN. Fourth Edition. Cr. 8v0. 6s.

Ryley (M. Beresford). QUEENS OF THE RENAISSANCE. With 24 Illus trations. Demy 8vo. ros. 6d. net.

Sainsbury (Harrington), M.D., F.R.C.P. PRI N C P I A T E R P E UTICA. Demy 8vo. 7s. 6d. net. See also New Library of Medicine.

St. Anselm. See Library of Devotion.

St. Atgustine. See Library of Devotion.

St. Bernard. See Library of Devotion.
St. Cyres (Viscount) See Oxford Bio. graphies.

St. Francis of Assisi. THE LITTLE FLOWERS OF THE GLORIOUS MESSER, AND OF HIS FRIARS. Done into English, with Notes by WILLIAM HEYwOOD. With 40 Illustrations from Italian Painters. Demy 8vo. 5s. net. See also Library of Devotion and Standard Library.

St. Francis de Sales. See Library of Devotion.

St. James. See Churchman's Bible and IVestminster Commentaries.

St. Luke. See Junior School Books.

St. Mark. See Junior School Books and Churchman's Bible.

St. Mattliew. See Junior School Books.

st. Paul. SECOND AND THIRD EPISTLES OF PAUL THE APOSTLE TO THE CORINTHIANS. Edited by James Houghton Kennedy, D.D., Assis tant Lecturer in Divinity in the University of Dublin. With Introduction, Dissertations, and Notes by J. SchmirT. Cr. 8ro. 6s. See also Churchman's: Bible and IVestminster Commentaries.

'Saki' (H. Munro). REGINALD. Second Edition. Ficap. 8vo. 2s. 6d. net.

Salmon (A. L.). See Little Guides.

Sanders (Lloyd). TH E H O L A N D HOUSE CIRCLE. With 24 Illustrations.

Second Edition. Demy 8vo. I2s. 6d. net. Sathas (C.). See Byzantine Texts.

Schmitt (John). See Byzantine Texts.

Schofield (A. T.), M.D., Hon. Phys. Freiden. ham Hospital. See New Library of Medicine.

Scudamore (Cyril). See Little Guides.

Scupoli (Dom. L.). See Library of Devotion.

Ségur (Madame de). See Simplified French Texts.

Sélincourt (E. de.) See Keats (John).

Sélincourt (Hugh de). GREATRALEGH. With I6 Illustrations. Demy 8vo. Ios. $6 d$. net.

Sells (V. P.), M.A. THE MECHANICS OF DAILY LIFE. Illustrated. $C r .8 v 0$. 2s. $6 d$.

Selous (Edmund). TOMMY SMITH'S ANIMALS. Illustrated by G. IV. ORD. Eleventh Fdition. Fcap. 8vo. 2s. 6d. School Edition, is. $6 d$.

TOMMY SMITH'S OTHER ANIMALS. Illustrated by Augusta GuEst. Fifth Edition. Fcap. 8vo. 2s 6d. School Edition, Is, 6 d.

Senter (George), B.Sc. (Lond.), Ph.D. See Textbooks of Science.

Shakespeare (William).

THE FOUR FOLIOS, I623; I632 ; ז664; 1685. Each $\ell_{4}, 4 s$. net, or a complete set, fir, i2s. net.

Folios 2, 3 and 4 are ready.

THE POEMS OF WILLIAM SHAKESPEARE. With an Introduction and Notes 
by George Wyndham. Demy 8vo. Buck. ram, gilt top, ros. $6 d$.

See also Arcten Shakespeare, Standard Library and Little Quarto Shakespeare.

Sharp (A.). VICTORIAN POETS. $C r$. 8vo. 2s. 6 .

Sharp (Cecil). See Baring-Gould (S.).

Sharp(Elizabeth). See Little Pooks on Art.

Shedlock (J. S.) THE PIANOFORTE SONATA. Cr.8\%o. $5^{s}$.

Shelley (Percy B.). See Standard Library. Sheppard (H. F.), M.A. See Baring. Gould (S.).

Sherwell (Arthur), M.A. LIFE IN IVEST LONDON. Third Edition. Cr. 8vo. $2 s .6 d$.

Shipley (Mary E.). AN ENGLISH CHURCH HISTORY FOR CHILD. REN. With a Preface by the Bishop of Gibraltar. With Maps and Illustrations. Cr. 8vo. Each part 2s. 6\%. net.

PART I.-To the Norman Conquest.

PART II.-To the Reformation.

Sichel (Walter). See Oxford Biographies.

Sidgwick (Mrs. Alfred). HONE LIFE IN GERMANY. With I6 Illustrations. Sccond Fdition. Demy 8vo. Ios. 6d. net.

Sime (John). See Iittle Books on Art.

Simonson (G. A.). FR A N E SCO GUARDI. With 4 I Plates. Imperial 4to. $\delta 2,2 s$. net.

Sketchley (R. E. D.). See Little Books on Art.

Skipton (H. P. K.). See Little Books on Art.

Sladen (Douglas). SICILY: The New Winter Resort. With over 200 Illustrations. Second Edition. Cr. 8vo. 5s. net.

Smallwood (M. G.). See Little Books on Art.

Smedley (F. F.). See I.P.L.

Smith (Adam). THE WEALTH OF NATIONS. Edited with an Introduction and numerous Notes by EDIVIN CANNAN, MI.A. Truo zolumes. Demy 8vo. 2is. net.

Smith (H. Bompas), M.A. A N E VV JUNIOR ARITHMETIC. Crown Svo. Vithout Answers, 2s. With Answers, 2s. 6 .

Smith (H. Clifford). See Connoisseur's Library.

Smith (Horace and James). See Little Library.

Smith (R. Mudie). THOUGHTS FOR THE DAY. Edited by. Farp. Bvo. 3s. 6d. net.

Smith (Nowell C.). See Wordsworth (W).

Smith (John Thomas). A BOOK FOR

A RAINY DAY: Or, Recollections of the Events of the Years I766-I833. Edited by WiLFRED WHITTEN. Illustrated. Widc Dcmy 8vo. I2s. 6d. net.

Snell (F. J.). A BOOK OF EXMIOOR. Illustrated. Cr. 8vo. $6 s$.

Snowden(C. E.). A HANDY DIGEST OF BRITISH HISTORY. Demy 8vo. 4s. 6 .

Sophocles. See Classical Translations.

Sornet (L. A.), and Acatos (M. J.) See Junior School Books.
Southey (R.). ENGLISH SEAMEN Edited by DAvid HANNAY.

Vol. I. (Howard, Clifford, Hawkins, Drake, Cavendish). Second Edition. Cr. 8ro. 6s.

Vol. II. (Richard Hawkins, Grenville, Essex, and Raleigh). Cr. 8ro. 6s.

See also Standard Library.

Souvestre (E.). See Simplified French Texts. Spence (C. H.), M.A. See School Examination Series.

Spicer (A. Dykes), M.A. THE PAPER TRADE. A Descriptive and Historical Survey. With Diagrams and Plans. Demy 8v0. I2s. $6 \%$. net.

Spooner (W. A.), M.A. See Leaders of Religion.

Spragge (W. Horton), M.A. See Junior School Eooks.

Staley (Edgcumbe). THE GUILDS OF FLORENCE. Illustrated. Second Edition. Royal8vo. I6s. net.

Stanbridge (J. W.), B.D. See Library of Devotion.

'Stancliffe.' GOLF DO'S AND DONT'S. Second Edition. Fcap. 8zo. Is.

Stead (D. W.). See Gallaher (D.).

Stedman (A. M. M.), M.A.

INITIA LATINA: Easy Lessons on Elementary Accidence. Eleventh Edition. Fcap. 8ro. Is.

FIRST LATIN LESSONS. Eleventh Edition. Cr. 8ro. $2 s$.

FIRST LATIN READER. With Notes adapted to the Shorter Latin Primer and Vocabulary. Seventh Edition. I8mo. Is. $6 d$.

EASY SELECTIONS FROM CASAR. The Helvetian War. Fourth Edition. I\&mo. Is.

EASY SELECTIONS FROM LIVY. The Kings of Rome. Second Edition. I8mo. Is. 6 \%

EASY LATIN PASSAGES FOR UNSEEN TRANSLATION. Truelfth Ed. Fcap. 8vo. Is. $6 d$.

EXEMPLA LATINA. First Exercises in Latin Accidence. With Vocabulary. Fourth Edition. Cr. 8vo. Is.

EASY LATIN EXERCISES ON THE SYNTAX OF THE SHORTER AND REVISED LATIN PRIMER. With Vocabulary. Twelfth Edition. Cr. Szo. Is. 6 . KEY, 3s. net.

THE LATIN COMPOUND SENTENCE: Rules and Exercises. Second Edition. Cr. 8\%o. Is. 6\%. With Vocabulary. 2s.

NOTANDA QUAEDAM: Miscellaneous Latin Exercises on Common Rules and Idioms. Fifth Edition. Fcap. 8vo. Is. 6d. With Vocabulary. 2s. KEY, 2s. net.

LATIN VOCABUULARIES FOR REPETITION: Arranged according to Subjects. Sixteenth Edition. Fcap. $8 v 0$. Is. $6 d$.

A VOCABULARY OF LATIN IDIOMS. I8mo. Fourth Edition. Is. 
STEPS TO GREEK. Fourth Edition. I8mo. Is.

A SHORTER GREEK PRIMER. Third Edition. Cr. 8vo. Is. 6 d.

EASY GREEK PASSAGES FOR UNSEEN TRANSLATION. Fourth Edition, re. vised. Ficap. 8vo. Is. 6 .

GREEK VOCABULARIES FOR REPETITION. Arranged according to Subjects. Fourth Edition. Fcap. 8vo. is 6\%. GREEK TESTAMENT SELECTIONS. For the use of Schools. With Introduction, Notes, and Vocabulary. Fourth Edition. Fcap. 8vo. 2s. 6 d.

STEPS TO FRENCH. Ninth Edition. $18 \mathrm{mo} .8 d$.

FIRST FRENCH LESSONS. Ninth Edi. tion. Cr. 8\%o. Is.

EASY FRENCH PASSAGES FOR UN. SEEN TRANSLATION. Sixth Edition. Fcap. 8\%o. Is. 6d.

EASY FRENCH EXERCISES ON ELE. MENTARY SYNTAX. With Vocabulary. Fourth Edition. Cr. 8vo. 2s.6d. KEY. 3s. net.

FRENCH VOCABULARIES FOR REPETITION : Arranged according to Subjects. Fourteenth Edition. Fcap. 8vo. Is. See also School Examination Series.

Steel (R. Elliott), M.A., F.C.S. THE WORLD OF SCIENCE. With I47 Illustrations. Second Edition. Cr. 8vo.2s.6d. See also School Examination Series.

Stephenson (C.), of the Technical College, Bradford, and Suddards (F.) of the Yorkshire College, Leeds. A TEXTBOOK DEALING WITH ORNAMENTAL DESIGN FOR WOVEN FABRICS. With 66 full-page Plates and numerous Diagrams in the Text. Third Edition. Demy 8vo. 7 s. 6 d.

Sterne (Laurence). See Little Library.

Steuart (Katherine). BY A L A N WATER. Second Edition. Cr. 8vo. $6 \mathrm{~s}$.

RICHARD KENNOWAY AND HIS FRIENDS. A Sequel to "By Allan Water.' Demy 8vo. 7s.6d. net.

Stevenson (R. L.) THE LETTERS OF ROBERT LOUIS STEVENSON TO HIS FAMILY AND FRIENDS. Selected and Edited by SIDNEy Colvin. Eighth Edition. 2 vols. $C r$.8vo. I2s.

VAILIMA LETTERS. With an Etched Portrait by William STRang. Seventh Edition. Cr. 8vo. Buckram. 6s.

THE LIFE OF R. L. STEVENSON See Balfour (G.).

Stevenson (M. I.). FROM SARANAC TO THE MARQUESAS. Being Letters written by Mrs. M. I. Stevenson during I887.88. Cr. 8vo. 6s. net.

LETTERS FROM SAMOA, I891.95. Edited and arranged by M. C. BALFour With many Illustrations. Second Edition $\mathrm{Cr}$. 8vo. 6s. net.

Stoddart (Anna M.). See Oxford Bio. graphies.
Stokes (F. G.), B.A. HOURS WITH RABELAIS. From the translation of SIR T. URquhart and P. A. Motreux. With a Portrait in Photogravure. Cr. 8vo. $3 s .6 d^{2}$. net.

Stone (S. J.). POEMS AND HYMNS. With a Memoir by F. G. Ellerton, M.A. With Portrait. Cr. 8vo. $6 s$.

Storr (Vernon F.), M.A., Canon of Win. chester. DEVELOPMENT AND DIVINE PURPOSE Cr. 8vo. 5s. net.

Story (Alfred T.). A M E R I C A N SHRINES IN ENGLAND. With 4 Il. lustrations in Colour, and Ig other Illustrations. Crown 8vo. $6 s$.

See also Little Guides.

Straker (F.). See Books on Business.

Streane (A. W.), D.D. See Churchman's Bible.

Streatfeild (R. A.). MODERN MUSIC AND MUSICIANS. With 24 Illustra. tions. SecondEd. Demy8vo. 75.6d. net. See also New Library of Music.

Stroud (Henry), D.Sc., M.A. ELEMEN TARY PRACTICAL PHYSICS. With II5 Diagrams. Second Edit., revised. Cr. 8vo. 4s. 6d.

Sturch (F.), Staff Instructor to the Surrey County Council. MANUAL TRAINING DRAWING (WOODWOR K). With Solutions to Examination Questions, Orthographic, Isometric and Oblique Projection. With 50 Plates and 140 Figures. Foolscap. 5s. net.

Suddards (F.). See Stephenson (C.).

Surtees (R. S.). See I.P. L.

Sutherland (William). OLD AGE PEN. SIONS IN THEORY AND PRACTICE, IITH SOME FOREIGN EXAMPLES. Cr. $8 v 0$. 3 s. 6 d. net.

* Swanton (E. W.), Member of the British Mycological Society. FUNGI AND HOW TO KNOW THEM. With I6 Coloured Plates by M. K. Spittal, and 32 Black and White Plates. Cr. 8vo. 5s. net.

Symes (J. E.), M.A. THE FRENCH REVOLUTION. Second Edition. Cr.8vo. 2s. $6 d$.

Sympson (E. Mansel), M.A., M.D. See Ancient Cities.

Tabor (Margaret E.). 'I'HE SAINTS IN ART. With 20 Illustrations. Fcap. 8vo. 3. $6 d^{2}$. net.

Tacitus. AGRICOLA. Edited by R. F. DAvis, M.A. Cr. 8vo. 2s.

GERMANIA. By the same Editor. $C r$. 8v0. 2s. See also Classical Translations.

Tallack (W.). HOWARD LETTERS AND MEMORIES. Demy 8vo. Ios. 6d. net.

Tatham (Frederick). See Blake (William). Tauler (J.). See Library of Devotion.

Taylor (A. E.). THE ELEMENTS OF METAPHYSICS. Second Edition. Demy 8vo. Ios. 6i. net.

Taylor (F. G.), M.A. See Commercial Series. Taylor (I. A.). See Oxford Biographies. 
Taylor (Jolın W.). THE COMING OF THE SAINTS. With 26 Illustrations. Demy 8vo. 7s. 6d. net.

Taylor (T. M.), M.A., Fellow of Gonville and Caius College, Cambridge. A CONSTITUTIONAL AND POLITICAL HISTORY OF ROME. To the Reign of Domitian. Cr.8vo. $7 s .6 d$.

Teasdale-Buckell (G. T.). THE COM. PLETE SHOT. With 53 Illustrations. Third Edition. Deny 8vo. I2s. 6d. net. Tennyson (Alfred, Lord). EARLY POEMS. Edited, with Notes and an Introduction, by J. CHurton COLlins, M.A. Cr. 8ro. $6 s$.

IN MEMORIAM, MAUD, AND THE PRINCESS. Edited by J. ChuRTON Collins, M.A. Cr. 8vo. $6 s$.

See also Little Library.

Terry (C. S.). See Oxford Biographies.

Terry (F. J.), B.A. ELEMENTARY LATIN. Cr. 8vo. 2s.

TEACHER'S HANDBOOK TO ELEMEN TARY LATIN. Containing the necessary supplementary matter to Pupil's edition. Cr. 8vo. 3s. 6d. net.

Thackeray (W. M.). See Little Library.

Theobald (F. V.), M.A. INSECT LIFE Illustrated. Second Edition Revised. Cr. 8vo. 2s. $6 d$.

Thibaudeau (A. C.). BONAPARTL $\Lambda N I$ THE CONSULATE. Translated and Edited by G. K. ForTesque, LL.D. With I2 Illustrations. Demy 8vo. ros. 6d. net.

Thompson (A. H.). See Little Guides.

Thompson (Francis). S E I, E C T E D POEMS OF FRANCIS THOMPSON. With a Biographical Note by WILFRID Meynel. With a Portrait in Photo. gravure. Second Edition. Faxp. Swo. 5s. net.

Thompson (A. P.). See 'lextbooks of Technology.

* Thomson (J. M.), Iellow and Deall of Divinity of Migdalen College, Oxford. JESUS ACCORDING 'TO ST. MARK. Cr. 8vo. 5 s.

Tileston (Mary W.). DAILY STRENGTH FOR DAILY NEEDS. Sixteenth Edi tion. Medinm i6mo. 2s. 6d.net. Also au edition in stıperior binding. $6 s$.

Tompkins (H. W.), F.R.II.S. See Little Books on Art and Little Guides.

Toynbee(Paget), MI.A., D.litt. IN THI FOOTPRINTS OF DAN'TE. A 'Trea sury of Verse and Prose from the works of Dante. Small Cr.8vo. 4s. 6 d. net.

DANTE IN ENGLISH LITERATURE: FROM CHAUCER TO CARY. TzUO vols. Demy 8vo. 2is. net.

See also Oxford Jiographies and Dante.

Tozer (Basil). 'THE HORSE' IN HIS. TORY. With 25 Illustrations. Cr. 820. $6 s$.

Tremayne (Eleanor E.). See Romantic History.

Trench (Herbert). DEIRDRE WEDDED, AND OTHER POXMs. Second and Rovised Edition. Large P'ost sao. 6s.
NEWV POEMS. Second Edition. Large Post 8vo. 6s.

APOLLO AND THE SEAMAN. Large Post 8vo. Paper, Is. 6a. net; cloth, 2s 6d. net.

Trevelyan(G. M.), Fellow of Trinity College, Cambridge. ENGLAND UNDER THE STUARTS. With Maps and Plans. Trirct Edition. Demy 8vo. Ios. 6d. net.

IENGLISH LIFE THREE HUNDRED YEARS $\triangle \mathrm{CrO}$ : Being the first two chapters of England uncier the Stuarts. Edited by J. TurRal. L.A. Cr. 8vo. is.

Triggs (lnigo H.), A.R.I.B.A. TOWN PLA N N I N ' ${ }^{\circ}$ : PAST, PRESEMT, A:D Possible. With i73 Illustrations. Wide Royal 8uo. I5s. net.

Troutbeck (G. E.). See Little Guides.

Tyler (E. A.), B.A., F.C.S. See Junior School Books.

Tyrrell=Gill (Frances). See Little Boolss on Art.

Unwin (George). See Antiquary's Books.

Vardon (Harry). TIIE COMIPL IE I I: GOLFER. With 63 Illustrations. Tenth Edition. Demy 8vo. Ios. 6u. net.

Vaughan (Henry). See Little Library.

Vaughan (Herbert M.), B.A.(Oxon.). THE LAST OF 'THE ROYAL STUARTS, HENRY S'IUART, CARDINAL, DUKE OF YORK. With 20 Illustrations. Second Edition. Demy 8vo. Ios. 6d. net.

'THE MENICI POPES (LEO X. AND CLEIIENT VII. With 20 Illustrations. Demy 8:10. I5s. net.

THE NAPLES RIVIERA. With 25 Illustrations in Colour by MAURICE GREIFFENHAGEN. Second Edition. Cr. Sao. $6 s$.

Vernon (Hon. W. Warrea), M.A. READINGS ON THE INFERNO OF DANTE. With an Introduction by the Rev. Dr. Moore. In Truo Volumes. Second Edition. Cr. 8zio. I Is. net.

READINGS ON THE PURGATORIO OF DANTE. Wilh an Introduction by the late Dean Churcir. In 'Yruo I olumes. Third Enition. Cr. Ero. I5s. net.

READINGS ON 'IIHE PARADISO OF DANTE. With an Introduction by the Bishop of Ripon. In Two Volumes. Sccond Edition. Cr. 8ro. I s. Het.

Vincent (J. E.). T If R OUGH EAS'T ANGLIA IN A MOTOK CAR. With I6 Illustrations in Colour by FrANk South. GATE, R.B.A., and a Map. Cr. 8vo. $6 s$.

Voegelin (A.), M.A. See Junior Examination Series.

Waddell (Col. L. A.), LI.. D., C.E. LHASA AND ITS MVSTLRIES. With a Record of the Expedition of $1903^{-1904}$. With 155 Illustrations and Maps. Third and Cheaserlidition. Medium 8vo. 7s. 6a. net.

Wade (G. W. ), D.D. OLD'TESTAMENT HIS'ORl. With Maps. Sixth Edition. Cr. 8zo. 6s.

Wade (G. W.), D.D., and Wade (J. H.), MI.A. See Litule Guides.

Vagner (Richard). RICHARD WAG- 


\section{General Literature}

NER'S MUSIC DRAMAS: Interpretations, embodying Wagner's own explanations. By Alice Leighton Cleather and Basil Crump. In Three Volumes. Fcap 8vo. 2s. 6d. each.

Vol. I.-The Ring of the Nibelung. Third Edition.

VOL. II.-PARSIFAL, LOHENGRIN, and The Holy Grail.

VOL. III. - TRISTAN AND ISOLDE.

Waimeman (Paul). A SUMMER TOUR IN FINLAND. With I6 Illustrations in Colour by Alexander Federley, i 6 other Illustrations and a Map. Demy 8vo. Ios. 6r. net.

Walkley (A. B.). DRAMA AND LIFE. Cr. 8vo. 6s.

Wall (J. C.). See Antiquary's Books.

Wallace-Hadrill (F.), Second Master at Herne Bay College. REVISION NOTES ON ENGLISH HISTORY. Cr. 8vo. Is.

Walters (H. B.). See Little Books on Art and Classics of Art.

Walton (F. W.), M.A. See School Histories.

Walton (lzaak) and Cotton (Charles). See I.P.L. and Little Library.

Waterhouse (Elizabeth). WITH THE SIMPLE-HEARTED : Little Homilies to Women in Country Places. Second Edition. Small Pott 8vo. 2s. net.

COMPANIONS OF THE WAY. Bein Selections for Morning and Evening Reading. Chosen and arranged by ELIZABETH WATERHOUSE. Large Cr. 8vo. 5s. net.

THOUGHTS OF A TERTIARY. Small Pott 8vo. is. net.

See also Little Library.

Watt (Francis). See Henderson (T. F.).

Weatherhead (T. C.), M.A. EXAMINATION PAPERS IN HORACE. Cr. 8vo.2s. See also Junior Examination Series.

*Webb (George W.), B.A. A SYS'TEMATIC GEOGRAPHY OF THE BRITISH ISLES. With Maps and Diagrams. $C r$. 8vo. Is.

Webber (F. C.). See Textbooks of Technology.

*Weigall (Arthur E. P.). A GUIDE TO THE ANTIQUITIES OF UPPER EGYPT: From Abydos to the Sudan Frontier. With 67 Maps, and Plans. Cr. 8vo. 7s. 6d. net.

Weir (Archibald), M.A. AN INTRO. DUCTION TO THE HISTORY OF MODERN EUROPE. Cr. 8vo. $6 s$.

Welch (Catharine). THE L I T T L E DAUPHIN. With I6 Illustrations. $C r$. 8vo. 6s.

Wells (Sidney H.) See Textbooks of Science.

Wells(J.), M.A. Fellow and Tutor of Wadham College. OXFORD AND OXFORD LIFE. Third Edition. Cr. 8vo. 3s. 6 d.

A SHORT HISTORY OF ROME. Ninth Edition. With 3 Maps. Cr. 8vo. $35.6 \%$. See also Little Guides.

Vesley (John). See Library of Devotion.

Westell (W. Percival). THE YOUNG NATURALIST. With 8 Coloured Plates by C. F. NEwALL, and many other Illustrations. Cr. Evo. 6s.

Westell (W. Percival), F.L.S., M.B.O.U. and Cooper (C. S.), F.R.H.S. THE YOUNG BOTANIS $\Gamma$. With 8 Coloured and 63 Plack and White Plates drawn from Nature, by C. F. Newall. Cr. $8 z^{\prime} 0.35 .6 d$. net.

Whibley (C.). See Henley (W. E.).

Whibley (L.), M.A., Fellow of Pembroke College, Cambridge. GREEK OLIGAR. CHIES : THEIR ORGANISATION AND CHARACTER. Cr. 8vo. 6s.

White (Eustace E.). THE COMPLETE HOCKEY PLAYER. With 32 Illustrations. Second Edition. Demy8vo. 5s.net. White (George F.), Lieut.-Col. A CEN. TURY OF SPAIN AND PORTUGAL. Demy 8vo. I2s. 6d. net.

White (Gilbert). See Standard Library.

Whitfield (E. E.), M.A. See Commercial Series.

Whitehead (A. W.). GASPARD D I COLIGNY, ADMIRAL OF FRANCE. With 26 Illustrations and ro Maps and Plans. Demy 8vo. 12s. 6\%.net.

Whiteley (R. Lloyd), F.I.C., Principal of the Municipal Science School, West Bromwich. AN ELEMENTARY TEXT. BOOK OF INORGANIC CHEMISTRY. Cr. 8vo 25. 6 d.

Whitley (Miss). See Dilke (Lady).

Whitling (Miss L.), late Staff Teacher of the National Training School of Cookery. THE COMPLETE COOK. With 42 Illustrations. Demy 8vo. 7s. 6d. net.

Whitten (W.). See Smith (John Thomas).

Whyte (A. G.), B.Sc. See Books on Business.

Wilberforce (Wilfrid). See Little Books on Art.

Wilde (Oscar). DE PROFUNDIS. Truelfth Edition. Cr. 8vo. 5s. net.

THE WORKS OF OSCAR WILDE. It 12 Volumes. Fcap. 8vo. 5s. net each wolume.

1. The Duchess of Padua. II. Lady WINDERMERE'S FAN. III. A WOMAN OF No IMPORTANCE. IV. THE IMPORTANCE OF BEING EARNEST. V. AN IDEAL HUSBAND. VI. DE PROFUNDIS and Prison LETTERS. VII. INTENTIONS. VIII. EsSAYS. IX. A House of Pomegranates. $x$. LORd Arthur Savile's Crime and the Portrait of Mr. W. H. Xi. Poems. xit. Salome, A Florentine Tragedy, and La SaInte Courtisane

Wilkins (W. H.), B.A. THE ALIEN INVASION. Cr. $8 v o .2 s .6 d$.

Williams (H. Noel), THE W O M E N BONAPARTES. The Nother and three Sisters of Napoleon. With 36 Illustrations. In Two Volumes Demy 8vo. 24 s net.

A ROSE OF SAVOY : MARIE ADELFIDE OF Savoy, Duchesse de Bourgogne, Mother OF LouIs xv. With a Frontispiece in Photogtavure and 16 other Illustrations. Demy 8vo. 15s. net. 
Williams (A.). PETROL PETER: or Pretty Stories and Funny Pictures. Illustrated in Colour by A. W. Mrlls. Demy 4to. 3s. 6d. net.

Williamson (M. G.)., M.A. See Ancient Cities.

Williamson (W.), B.A. See Junior Ex. amination Series, Junior School Books, and Beginner's Books.

Wilmot-Buxton (E. MI.), F. R. Hist. S. MAKERS OF EUROPE. Outlines of European History for the Middle Forms of Schools. With r2 Maps. Tenth Edition. Cr. 8\%0. 3s. 6d.

THE ANCIENT WORLD. With Maps and Illustrations. Cr.ero. 35.6 .

A BOOK OF NOBLE WOMEN. With I6 Illustrations. Cr. E*o. 3s. $6 d$.

A HISTORY OF GREAT BRITAIN : FROM THE COMING OF THE A.NGLES TO THE YEAR I87o. With 20 Maps. Cr. Evo. 35. 6d. See also Beginner's Books and New Historical Series.

Wilson(Bishop.). See Library of Devotion.

Wilson (A. J.). See Books on Business.

Wilson (H. A.). See Bools on Business.

Wilton (Richard), M.A. LYRA PAS. TORALIS: Songs of Nature, Church, and Home. Pott 8vo. 2s. 6d.

Winbolt (S. E.), M.A. EXERCISES IN LATIN ACCIDENCE. Cr. \&vo. Is. 6d.

LATIN HEXAMETER VERSE: An Aid to Composition. Cr. $8 v 0.35 .6 d^{2}$. KEY, 5. net.

Windle (B. C. A.), D.Sc.,F.R.S., F.S.A. See A'liquary's Books, Little Guides, Ancient Cities, and School Histories.

Wood (Sir Evelyn), F. M., V.C., G.C.B., G.C.M.G. FROM MIDSHIPMAN TO FIELD-MARSHAL. With Illustrations, and 27 Maps. Fifth and Cheaper Edition. Demy E:0. 75. Ed. net.

THE REVOLT IN HINDUSTAN. Is $57^{\circ}$ 59. With 8 Illustrations and 5 Maps. Second Edition. Cr. 8vo. 6s.

Wood (J. A. E.). See Textbooks of Technology:

Wood (J. Hickory). DAY LENO. Illustrated. Third Edition. Cr. 8vo. $6 \mathrm{~s}$.

Wood (W. Birkbeck), M.A., late Scholar of Worcester College, Oxford, and Edmonds (Major J. E.), R.E., D.A.Q.-M.G. A HISTORY OF THE CIVIL WAR IN
THE UNITED STATES. With an Introduction by H. SPENSER WILK1:SON. With 24 . Maps and Plans. Second Edition. Demy 870. 12s. 6d. net.

Wordsworth (Christopher), M.A. See Antiquary's Books.

Wordsworth (W.). THE POEMS OF. With an Introduction and Notes by Nowell C. Surth, late Fellow of New College, Oxford. In Three Volumes. Demy 8vo. 15s. net.

POEMS BY WILIIAM WORDSWORTH. Selected with an Introduction by STOPFORD A. BroOKe. With 40 Illustrations by $\mathrm{E}$. H. NEW, including a Frontispiece in Photogravure. Cr. 8vo. 7s. 6d. net. See also Little Library.

Wordsworth (W.) and Coleridge (S. T.). See Little Library.

Wright (Arthur), D.D., Fellow of Queen's College, Cambridge. See Churchman's Library.

Wright (C. Gordon). See Dante.

Wright (J. C.). TO-DAY. Thoughts on Life for every day. Demy i6mo. Is.6d. net.

Wright (Sophie). GERMAN VOCABL LARIES FOR REPETITION. Fcap. Ẽc. Is. 6 ?

Wvatt (Kate M.). See Gloag (M. R.).

Wylde (A. B.). MODERN ABYSSINIA. With a Map and a Portrait. Demy 8vo. I5s. net.

Wyllie (M. A.). NORIVAY A.TD ITS FJORDS. With I 6 Illustrations, in Colour by W. L. WYLLIE, R.A., and i7 other Illustrations. Crown \&vo. 6s.

Wyndham (George). See Shakespeare (William).

Yeats (IV. B.). A BOOK OF IRISH VERSE. Rezised and Enlarged Edition. Cr. Evo. 3s. 6d.

Young (Filson). THE COMPLETE MOTORIST. With I 38 Illustrations. New Edition (Serenth), ruith many additions. Dem\% 8ro. I2s. 6it. net.

THE JOY OF THE ROAD : An Apprecia. tion of the Motor Car. With a Frontispiece in Photogravure. Small Demy 8vo. 5s. net.

Zachariah of Mitylene. See Byzantine Texts.

Zimmern (Antonia). WHAT DO WH KNOW CONCERNING ELECTRI. CITY? Fcap. 8vo. Is. 6a. net.

\section{Ancient Cities}

General Editor, B. C. A. WINDLE, D.Sc., F.R.S.

$$
\text { Cr. Sio. 4s. 6a. net. }
$$

Bristol. By Alfred Harvey, M.B. Illus-|Edinburgh. By M. G. Williamson, M.A. trated by E. H. New.

CANTERBURY. By J. C. Cox, LL.D., F.S.A Illustrated by B. C. Boulter.

Chester. By B. C. A. Windle, D.Sc. F.R.S. Illustrated by E. H. New.

Dublix. ByS.A. O. Fitzpatrick. Illustrated by W. C. Green. Illustrated by Herbert Railton.

Liscols. By E. Mansel Sympson, M.A., M.D. Illustrated by E. H. New

Shreivsbury. By T. Auden, M1.A., F.S.A. Illustrated by Katharine M. Roberts.

Wells and Glastonirury. By T. S. Holmes. Illustrated by E. H. New. 


\title{
The Antiquary's Books
}

\author{
General Editor, J. CHARLES COX, LL.D., F.S.A \\ Demy Svo. 7s. 6d. net.
}

ArChalogy and False Antiquities. By R. Munro, LL.D. With 8 I Illustrations. Bells of England, The. By Canon J.J. Raven, D.D., F.S.A. With 6o Illustrations, Second Edition.

Brasses of England, The. By Herbert W. Macklin, M.A. With 85 Illustrations. Second Edition.

Celtic Art in Pagan and Christian Times. By J. Romilly Allen, F.S.A. With 44 Plates and numerous Illustrations.

Domesday Inquest, Tire. By Adolphus Ballard, B.A., LL.B. With 27 Illustrations.

English Church Furniture. Py J. C. Cox, LL.D., F.S.A., and A. Harvey, M.B. With I2I Illustrations. Second Edition.

English Costume. From Prehistoric Times to the End of the Eighteenth Century. By George Clinch, F.G.S. With I3 I Illustra. tions.

English Monastic Life. By the Right Rev. Abbot Gasquet, O.S.B. With 5o Illustrations, Maps and Plans. Third Edition.

English Seals. By J. Harvey Bloom. With 93 Illustrations.

Folk-Lore as an Historical Science. By G. L. Gomme. With 23 Illustrations.

Gilds and Companies of London, The. By George Unwin. With 37 Illustrations.

Manor and Manortal Records, The. By Nathaniel J. Hone. With 54 Illustrations.

Mediaval Hospitals of England, The. By Rotha Mary Clay. With many Illus. trations.

Old Service Books of the English Church. By Christopher Wordsworth, M.A., and Henry Littlehales. With $3^{8}$ Coloured and other Illustrations.

PaRish LifE IN MEDIAVAL ENGLaNd. By the Right Rev. Abbott Gasquet, O.S.B. With 39 Illustrations. Second Edition.

Remains OF THE PREHistoric AgE IN ENGLAND. By B. C. A. Windle, D.Sc., F.R.S. With 94 Illustrations. Second Edition.

Royal. Forests of England, The. By J. C. Cox, LL.D., F.S.A. With 25 Plates and 23 other Illustrations.

Shrines of British Saints. By J. C. Wall. With 28 Plates and 50 other Illustrations.

\section{The Arden Shakespeare}

\section{Demy 8vo. 2s. 6 . net each volume.}

An edition of Shakespeare in single Plays. Edited with a full Introduction, Textual Notes, and a Commentary at the foot of the page.

All's Well That Ends Well. Edited by ${ }^{*}$ Macbeth. Edited by H. Cuningham.

W. O. Brigstocke.

Antony and Cleoratra. Edited by R. H. Case.

Cymbeline. Edited by E. Dowden.

Comedy of Errors, The. Edited by Henty Cuningham.

Hamlet. Edited by E. Dowden. Second Edition.

Julius Caesar. Edited by M. Macmillan.

King HENRY v. Edited by H. A. Evans.

King Henry vi. PT. I. Edited by H. C. Hart.

Kina Henry vi. Pt. 11. Edited by H. C. Hart and C. K. Pooler.

King Lear. Edited by W. J. Craig.

KING RICHARD III. Edited by A. $H$.

Thompson.
LifE AND DEATH OF King John, ThE. Edited by I vor B. John.

Love'S LABOUR'S I,Ost. Edited by H. C. Hart.

Measure for Measure. Edited by H. C. Hart.

Merchant of Venice, The. Edited by C. K. Pooler.

MerRy Wives of Windsor, The. Edited by H. C. Hart.

A Midsummer Night's Dream. Edited by $H$. Cuningham.

OTHELlo. Fitited by H. C. Hart.

Pericles. Edited by K. Deighton.

Romeo AND Juliet. Edited by Edward Dowden.

TAMing of the Shrew, The. Edited by R. Warwick Bond.

TEMPEST, THE. Edited by M. Luce.

Timon of Athens. Edited by K. Deighton.

Titus Andronicus. Edited by H. B. Baildon.

Troilus and Cressida. Edited by $K$. Deighton.

Two Gentlemen of Verona, The. Fdited by R. W. Bond.

Twelfth Night. Edited by M. Luce. 


\section{The Beginner's Books}

\section{Edited by W. WILLIAMSON, B.A.}

EAsy Dictation and SPELling. By W. Williamson, B.A. Serenth Ed. Fcap.8zo. Is.

Easy Exercises in Arithmetic. Arranged by W. S. Beard. Third Edition. Fcap. 8vo. Without Answers, Is. With Answers. Is. 3 d.

EAsy French Rhymes. By Henri Blouet. Second Edition. Illustrated. Fcap.8vo. Is.

AN EAsy POETRY Book. Selected and arranged by W. Williamson, B.A. Second Edition. Cr. 8vo. Is.

EAsy Stories from ENglish History. By E. M. Wilmot-Buxton, F.R.Hist.S. Fifth Edition. Cr.8vo. Is.

A First History of Greece. By E. E. Firth. With 7 Mlaps. Cr. 8vo. Is. $6 d$.

Stories from Roman History. By E. M. Wilmot-Buxton. Second Edition. Cr. 8vo. Is. $6 d$.

Stories From the Old Testament, By E. Wilmot-Buxton. Cr. 8v0. Is. $6 d$.

\section{Books on Business}

\section{Cr. 8vo. 2s. 6a. net.}

Automobile Industry, The. G. Holden. Law in Business. H. A. Wilson.

Stone.
REWING INDUSTRY, THE. J. L. Baker,

F.I.C., F.C.S. With 28 Illustrations.

Business of Advertising, The. C. G. Moran, With II Illustrations.

Business Side of Agriculture, ThE. A. G. L. Rogers.

Business of Insurance, The. A. J. Wilson.

Civil Engineering. C. T. Fidler. With I5 Illustrations.

Cotton Industry and Trade, The. S. J. Chapman. With 8 Illustrations.

The Electrical Industry : Lighting, Traction, and Power. A. G. Whyte,

Iron Trade of Great Britain, The. J. S. Jeans. With I2 Illustrations. Mining and Mining Investments. A.

Money Market, The. F. Straker.

Monopolies, Trusts, and Kartells. F. W. Hirst.

Ports And Docks. Douglas Owen.

Railways. E. R. McDermott.

Shipbuilding Industry The: Its History, Practice, Science, and Finance. David Pollock, M.I.N.A.

Stock Exchange, The. C. Duguid. Second Edition.

Trade Unions. G. Drage.

\section{Byzantine Texts}

\section{Edited by J. B. BURY, M.A., Litt.D.}

Tile Syriac Chronicle KNOWN AS THAT OF ZaChariah OF MitYlenE. Translated by F. J. Hamilton, D.D., and E. W. Brooks. Demy 8vo. I 25.6 d. net.

Evagrius. Edited by L. Bidez and Léon Parmentier. Demy svo. Ios. 6i. net.

The History of Psellus. Edited by C. Sathas. Demy 8vo. I5s. net.

ECTHESIS Chronica AND ChRONICON ATHENARUM. Edited by Professor S. P. Lambros. Demy 8vo. 7s. 6d. net.

The CHRONicle of MOREA. Edited by John Schmitt. Demy 8vo. I5s. net.

\section{The Churchman's Bible}

General Editor, J. H. BURN, B.D., F.R.S.E.

$$
\text { Fcap. Svo. Is. 6d. net each. }
$$

The Epistle of St. Paul, The Afostle to/ Isaiah. Explained by W. E. Barnes, D.D. ThE Galatians. Explained by A. W. Robinson, M.A. Second Eidition.

Ecclesiastes. Explained by A. W. Streane, D.D.

The Epistle of St. Paul the Arostle to the Philippians. Explained by C. R, D. Biggs, D.D. Second Lidition.

Tine Epistle of ST. JamLs. Explained by H. W. Fulford M.A. Tavo Volumes. With Map. 2s. net each.

The Epistle of St. Paul the Apostle to the Erhesians. Explained by G. H. Whitaker, M.A.

Thb Gospel According to St. Makk. Explained by J. C. Du Buisson, M.A. 2s. 6 d. net.

The Epistle of Paul tile Apostle to the Colossians and Philemon. Explained by H. J. C. Knight. as. net. 


\section{General Literature}

\section{The Churchman's Library}

General Editor, J. H. BURN, B.D., F.R.S.E.

Crown Svo. 3s. 6d. each.

The Beginnings of Englisil Christianity. By W. E. Collins, M.A. With Map.

The Churchman's Introduction to the Old Testament. By A. M. Mackay, B.A. Second Edition.

Evolution. By F. B. Jevons, M.A., Litt.D.

Some New Testament Problems. By Arthur Wright, D.D. $6 s$.

The Workmanship of ThE Prayer Book: Its Literary and Liturgical Aspects. By J. Dowden, D.D. Second Edition, Revised and Enlarged.

\section{Classical Translations}

Crown Svo.

AEschylus-The Oresteian Trilogy (Agamem. HokacE-The Odes and Epodes. Translated non, Choëphoroe, Eumenides). Translated by Lewis Campbell, LL. D. $5 s$.

CiCERO-De Oratore 1. Translated by E. N. P. Moor, M.A. Second Edition. 3s. 6 .

CiCERo-The Speeches against Cataline and Antony and for Murena and Milo. Trans. lated by H. E. D. Blakiston, M.A. $5 s$.

Cicero-De Natura Deorum. Translated by F. Brooks, M.A. $3 s .6 d$.

Cicero-De Officiis. Translated by G. B. Gardiner, M.A. 2s. $6 d$. by A. D. Godley, M.A. 2s.

LuCian-Six Dialogues Translated by S. T Irwin, M. A. 3 s. 6 .

SophoCles-Ajax and Electra. Translated by E. D. Morshead, M.A. $2 s .6 d$.

TAcitus-Agricola and Germania. Trans. lated by R. B. Townshend. 2s. 6 .

Juvenal-Thirteen Satires. Translated by S. G. Owen, M.A. $2 s .6 d$.

\section{Classics of Art}

Edited by DR. J. IJ. W. LAING

Wide Royal 8vo. Gilt top.

Thr Art of the Greeks. By H. B. Walters. Rubens. Edward Dillon, M.A. With With II2 Plates and IS Illustrations in the Text. I $2 s .6$. net.

Florentine Sculptors of the Rennais. ANCE. Wilhelm Bode, Ph.D. Translated by Jessie Haynes. With 94 Plates. I2s. 6d. net.

Ghirlandaio. Gerald S. Davies, Master of the Charterhouse. With 50 Plates. Second Edition. Ios. 6d.

* Michelangelo. Gerald S. Davies, Master of the Charterhouse. With 126 Plates. I2s. 6 d. net. Frontispiece in Photogravure and ${ }_{4} 83$ Plates. 25s. net.

RaphaEl. A. P. Oppé. With a Frontispiece in Photogravure and 200 Illustrations. I2s. 6 d. net.

Trtian. Charles Ricketts. With about 220 Illustrations. 12s. 6d. net.

Velazquez. By A. de Beruete. With 94 Plates. Ios. 6d. net.

\section{Commercial Series}

\section{Crowur 8vo.}

British Commerce and Colonies from A Short Commercial Arithmetic. Fy F. Elizabeth to Victoria. By H. de B. G. Taylor, M.A. Fourth Edition. is. 6d. Gibbins, Litt.D., M.A. Fourth Edition. 2s. French Conmercial Correspondence. By

Commercial Examination Papers. By H. de B. Gibbins, Litt.D., M.A. Is. 6 .

The Economics of Commerce, By $\mathrm{H}$. de B. Gibbins, Litt.D., M.A. Second Edition. Is. $6 d$.

A German Commercial Reader. By S. E. Bally. With Vocabulary. $2 s$.

A Commercial Geography of the Britisif EMPIRE. By L. W. Lyde, M.A. Sevent/ Edition. 2s.

A Commercial Geography of Foreign Nations. By F. C. Boon, B.A. $2 s$.

A Primer of Business. By S. Jackson, M.A. Fourth Edition. Is. 6 . S. E. Bally. With Vocabulary. Fiourth Edition. 2s.

German Commercial Corres pondence. By S. E. Bally: With Vocabulary. Secont Edition. 2s. $6 i$.

A French Commercial Reader. By S. E. Bally. With Vocabulary. Secont Eidition. 2s.

Precis Writing and OfFice CORRESPONDENCE. By E. E. Whitfield, M.A. Second Edition. 2s.

An Entrance Guide to Professions and Business. By H. Jones. Is. $6 d$.

The Principles of Book-keeping by Double EnTRY. By J. E. B. M'Allen, M.A. 2s.

Commercial Law. By W. Douglas Edwards. Second Edition. 2s. 


\section{Messrs. Methuen's Catalogue}

\section{The Connoisseur's Library \\ Wide Royal 8vo. 25s. net.}

Mezzotints. By Cyril Davenport. With $40 \mid$ European Enamels. By Henry H. Cunyng. Plates in Photogravure.

Porcrlain. By Edward Dillon. With ig Plates in Colour, 20 in Collotype, and 5 in Photogravure.

Miniatures. By Dudley Heath. With 9 Plates in Colour, 15 in Collotype, and 15 in Photogravure.

Ivories. By A. Maskell. With 80 Plates in Collotype and Photogravure.

English Furniture. By F. S. Robinson. With r6o Plates in Collotype and one in Photogravure. Second Edition.

English Coloured Books. By Martin Hardie. With 28 Illustrations in Colour and Collotype.

\section{Handbooks of English Church History}

Edited by J. H. BURN, B. D. Crown 8vo. 2s. 6d. net.

The Foundationsof the English Church. The Medieval Church and the Papacy J. H. Maude.

The Saxon Church and the Norman Con- *The Reformation Period. By Henty Quest. C. T. Cruttwel.l.

\section{The Illustrated Pocket Library of Plain and Coloured Books}

Fcap Svo. 3s. 6d. net each volume.

\section{COLOURED BOOKS}

Old Coloured Books. By George Paston. IVith I6 Coloured Plates. Ficap.8vo. 2s, net.

The Life and Death of John Mytton, EsQ. By Nimrod. With 18 Coloured Plates by Henry Alken and T. J. Rawlins. Fourth Edition.

The Life of a Sportsman. By Nimrod. With 35 Coloured Plates by Henry Alken. Handley Cross. By R. S. Surtees. With I7 Coloured Plates and roo Wondcuts in the Text by John Leech. Second Ediizon.

Mr. Sponge's Sporting Tour. By R. S. Surtees. With I $_{3}$ Coloured Plates and 90 Woodcuts in the Text by John Leech.

Jorrocks' Jaunts and Joli.ities. By R.S. Surtees. With I5 Coloured Plates by H. Alken. Second Edition.

Ask Mamma. By R. S. Surtees. With $x_{3}$ Coloured Plates and 70 Woodcuts in the Text by John Leech.

The Analysis of the Hunting Field. By R. S. Surtees. With 7 Coloured Plates by Henry Alken, and 43 Illustrations on Wood.

The Tour of Dr. Syntax in Search of the Picturesque. By IVilliam Combe. IVith 30 Coloured Plates by T. Rowlandson.

Tine Tour of Doctor Syntax in Searcil of Consolation. By William Combe. With 24 Coloured Plates by T. Rowlandson.

The Tilrd Tour of Doctor Syntax in Search of a IVIfE. By William Combe. With 24 Coloured Plates by T. Rowlandson.
The History of Johnny Quae Genus: the Little Foundling of the late Dr. Syntax. By the Author of "The Three Tours.' With 24 Coloured Plates by Rowlandson.

The English Dance of Death, from the Designs of T. Rowlandson, with Metrical Illustrations by the Author of 'Doctor Syntax.' Truo Volumes.

This book contains 76 Coloured Plates.

The Dance of Life: A Poem. By the Author of 'Doctor Syntax.' Illustrated with 26 Coloured Engravings by 'I. Rowlandson.

LiFE In London: or, the Day and Night Scenes of Jerry Hawthorn, Esq., and his Elegant Friend, Corinthian Tom. By Pierce Egan. With 36 Coloured Plates by I. R. and G. Cruikshank. With numerous Designs on Wood.

REAL LIFE IN LONDON: or, the Rambles and Adventures of Bob Tallyho, Esq., and his Cousin, The Hon. Tom Dashall. By an Amateur(Pierce Egan). With 3r Coloured Plates by Alken and Rowlandson, etc. Tro Volumes.

Tire Life of AN Actor. By Pierce Egan. With 27 Coloured Plates by Theodore Lane, and several Designs on Wood.

Tile Vicar of Wakefield. By Oliver Goldsmitl. With 24 Coloured Plates by T. Row landson.

Tile Military Adventures of Johnny Newcome. By an Officer. With I 5 Coloured Plates by T. Rowlandson. 


\section{GENERAL LiteratuRE}

Illustrated Pocket Library of Plain and Colourto Books-continued.

The National Sports of Great Britain. With Descriptions and 50 Coloured Plates by Henry Alken.

The Adventures of a Post Captain. By A Naval Officer. With 24 Coloured Plates by Mr. Williams.

Gamonia : or, the Art of Preserving Game; and an Improved Method of making Planta. tions and Covers, explained and illustrated by Lawrence Rawstorne, Esq. With I5 Coloured Plates by T. Rawlins.

An Academy for Grown Horsemen: Containing the completest Instructions for Walking, Trotting, Cantering, Galloping, Stumbling, and Tumbling. Illustrated with 27 Coloured Plates, and adorned with a Portrait of the Author. By Geoffrey Gambado, Esq.

\section{PIAIN BOOKS}

The Grave: A Poem. By Robert Plair. Jllustrated by 12 Etchings executed by Louis Schiavonetti from the original Inventions of William Blake. With an Engraved Title Page and a Portrait of Blake by'T. Phillips, R.A.

The illustrations are reprcduced in photogravure.

ILLUSTRATINNS OF THE BOOK OF JOB. Invented and engraved by William Blake.

These famous Illustrations $-2 I$ in number -are reproduced in photogravure.

Windsor Castle By IV. Harrison Ainsworth. With 22 Plates and 87 Woodcuts in the Text by George Cruikshank.
ReAl LifE in IRELAND, or, the Day and Night Scenes of Brian Boru, Esq., and his Elegant Friend, Sir Shawn O'Dogherty. By a Real Paddy. With Ig Coloured Plates by Heath, Marks, etc.

The Adventures of Johnny Newcome iv TIIE NAvy. By Alfred Burton. With I6 Coloured Plates by ' $\Gamma$. Rowlandson.

The Old English Squire: A Poein. By John Careless, Esq. With 20 Coloured Plates after the style of T. Rowlandson.

The ENGlish Spy. By Bernard Blackmantle. An original Work, Characteristic, Satirical, Humorous, comprising scenes and sketches in every Rank of Society, being Portraits of the Illustrious, Eminent, Eccentric, and Notorious. With 72 Coloured Plates by R. CRUIKSHANK, and many Illustrations on wood. Trwo Volumes. 7 s. net.

The Tower of London. By W. Harrison Ainsworth. With 40 Plates and 58 Woodcuts in the Text by George Cruikshank.

Frank Fairlegh. By F. E. Smedley. With 30 Plates by George Cruikshank.

HANDy Andy. By Samuel Lover. With 24 Illustrations by the Author.

The Compleat ANgler. By Izaak Walton and Charles Cotton. With 44 Plates and 77 Woodcuts in the Text.

The Pickwick Pal'ers. By Charles Dickens. With the 43 Illustrations by Seymour and Phiz, the two Buss Plates, and the 32 Contemporary Onwhyn Plates.

\section{Junior Examination Series}

Edited by A. M. M. STEDMAN, M.A. Fcap. 8vo. Is.

Junior Algebra Examination Papers. By Junior Geography Examination Papers. S. W. Finn, M.A.

Junior Arithmetic Examination Papers. By W.S. Beard. Fifth Edition.

Junior English Examination Papers. By W. Williamson, B.A. Second Edition.

Junior French Examination Papers. Py F. Jacob, M.A. Second Edition.

Junior General INFORMation Examination Papers. By W. S. Beard. Key, 3s. 6 d. net. By W. G. Baker, M.A.

Junior German Examination Papers. By A. Voegelin, M.A.

Junior Greek Examination Papers. By $T$. C. Weatherhead, M.A. KEY, 3s. 6d. net. Junior Latin Examination PAPERs. By C G. Botting, B.A. Sixth Edition. KEY, 3. $6 d$. net.

* Junior History Examination Papers. By W. O. P. Davis.

\section{Methuen's Junior School-Books}

Edited by O. D. INSKIP, LL.D., and W. WILLIAMSON, B.A.

A Ciass.Book of Dictation Passages. By W. Williamson, B.A. Fourteenth Edition. Cr. 8vo. Is. $6 d$.

The Gospel According to St. Matthew. Edited by E. Wilton South, M.A. With Three Maps. Cr. 8vo. Is. 6 .

The Gospel According ToSt. Mark. Edited by A. E. Rubie, D.D. With Three Maps. Cr. 8vo. Is. 6d.
A Junior English Grammar. By W. Williamson, B.A. With numerous passages for varsing and analysis, and a chapter on Essay Writing. Fourth Edition. Cr. 8vo. $2 s$.

A Junior Chemistry. By E. A. Tyler, B.A. F.C.S. With 78 Illustrations. Fourth Edition. Cr. 8vo. 2s. 6d.

The Acts of THE Apostles. Edited by A. E. Rubie, D.D. Cr. 8vo. 2s. 
Methuen's Junior Schoor, Books-continued.

A Junior French Grammar. By L. A. The Gospel According to St. Luke. With Sornet and M. J. Acatos. Third Edition. an Introduction and Notes by William Cr. 8vo. $2 s$.

Elementary Experimental. Science. Phy. sics by W. T. Clough, A.R.C.Sc. (Lond.), F.C.S. Chemistry by A. E. Dunstan, B.Sc. (Lond.), F.C.S. With 2 Plates and I54 Diagrams. Seventh Edition. Cr.8vo. 2s. $6 d$.

A Junior Geometry. By Noel S. Lydon. With 276 Diagrams. Seventh Edition. Cr. 8vo. 25 .

Elementary Experimental Chemistry. By A. E. Dunstan, B.Sc. (Lond.), F.C.S. With 4 Plates and rog Diagrams. Third Edition. Cr.8vo. 2s.

A Junior French Prose. By R. R. N. Paron, M.A. ThirdEdition. Cr.8vo. 2s. Williamson, B.A. With Three Maps. Cr. 8vo. $2 s$.

The First Book of Kings. Edited by A. E. Rubie, D.D. With 4 Maps. C $\% 8 v 0$. 25 . A Junior Greek History. By W. H. Spragge, M.A. With 4 Illustrations and 5 Maps. Cr. 8vo. 2s. 6d.

A School latin Grammar. By H. G. Ford, M.A. Cr. 8vo. 2s. 6 d.

A Junior latin Prose. Ey H. N. Asman, M.A., B.D. Cr. 8vo. 2s. 6 d.

*Elementary Experimental Electricity and Magnetism. By W. T. Clough, A.R.C.Sc. (Lond.), F.C.S. With 200 Illustrations and Diagrams. Cr. 8vo. $2 s .6 d$.

Englise ILiterature for Schools. By Edith E. Firth. Cr. 8vo. 2s. $6 d$.

\section{Leaders of Religion}

Edited by H. C. BEECHING, M.A., Canon of Westminster. With Portraits. Cr. 8vo. 2s. net.

Cardinal Newman. By R. H. Hutton. JOHN WESLEY. By J. H. Overton, M.A. Bishop Wilberforce. By G. W. Daniell, M.A.

Cardinal Manning. By A. W. Hutton, M.A. Charles Simeon. By H. C. G. Mloule, D.D. JoHN KNox. By F. MacCunn. Second Edition. JoHn Howe. By R. F. Horton, D.D.

Thomas Ken. By F. A. Clarke, M.A.

GeORGE Fox, THE QUAKER. ByT. Hodgkin, D.C.L. Third Edition.

JohN KEBLe. By Walter Lock, D.D.
Thomas Chalmers. By Mrs. Oliphant. LancFlot Andrewes. By' R. L. Ottley, D.D. Second Edition.

Augustine of Canterbury. By E. L. Cutts, D.D.

William Laud. By W. H. Hutton, M.A. Third Edition.

John Donne. By Augustus Jessopp, D.D. 'Thomas Cranmer. By A. J. Mason, D.D.

Bishop Latimer. By R. M. Carlyle and A. J. Carlyle, M.A.

Bishop Butler. By W. A. Spooner, M.A.

\section{The Library of Devotion}

With Introductions and (where necessary) Notes.

Small Pott Svo, cloth, 2s. ; leather, 2s. 6d. net.

The Conffessions of St. Augustine. Edited by C. Bigg, D.D. Sixth Edition.

The Imitation of Christ : called also the Ecclesiastical Music. Edited by C. Bigg, D.D. Fifth Editicn.

THE CHRISTIAN YEAR. Edited by Walter Lock, D.D. Fourt/ Edition.

LYRA INNOCENTIUM. Edited by Walter Lock, D.D. Second Edition.

The Temple. Edited by E. C. S. Gibson, D.D. Second Edition.

A Book of Devotions. Edited by J. W. Stanbridge. B.D. Second Edition.

a Serious Call to a Devout and Holy LifE. Edited by C. Bigg, D.D. Fourth Ed.

A Guide to Eternity. Edited by J. W. Stanbridge, B.D.

The Inner Way. By J. Tauler. Edited by A. W. Hutton, M. $\Lambda$.
On the Love of God. Ey St. Francis de Sales. Edited by W\%. J. Knox-Little, M.A.

The Psalms of David. Edited by B. W. Randolph, D.D.

Lyra Apostolica. By Cardinal Newman and others. Edited by Canon Scott Holland, M.A., and Canon H. C. Beeching: M.A.

The Song of Songs. Edited by B. Blaxland, M.A.

The Thoughts of Pascal. Edited by $C$. S. Jerram, M.A.

A Manual of Consolation from the Saints and Fathers. Edited by J. H. Burn, B.D.

* Devotions from the Apocrypha. Edited, with an Introduction, by Herbert Pentin, M.A. 
The Library of Devotion-continued.

*The Spiritual Combat. By Dom Lorenzo Scupoli. Newly translated, with an Introduction and Notes, by Thomas Barns, M.A.

The Devotions of St. Anselm. Edited by C. C. J. Webb, M.A.

Grace Abounding to the Chief of SinNERS. By John Bunyan. Edited by S. C. Freer, M.A.

Bishop Wilson's Sacra Privata. Edited by A. E. Burn, B.I).

LYRA SACRA: A Book of Sacred Verse. Edited by Canon H. C. Beeching, M.A. Second Edition, revised.

A Day Book From the Saints and Fathers. Edited by J. H. Burn, B.D.

A Little Book of Heavenly Wisdom. A Selection from the English Mystics. Edited by E. C. Gregory.

Light, Life, and Love. A Selection from the German Mystics. Edited byW.R.Inge, M.A.

An Introduction to The Devout Life. By St. Francis de Sales. Translated and Edited by T. Barns, M.A.

The Little Flowh of the Glorious Messer St. Francis and OF HIS Friars. Done into English by W. Heywood. With an Introduction by A. G. Ferrers Howell.
Manchester al MONDo: a Contemplation of Death and Immortality. By Henry Montagu, Earl of Manchester. With an Introduction by Elizabeth Waterhouse, Editor of 'A Little Book of Life and Death.'

The Spiritual Guide, which Disentangles the Soul and brings it by the Inward Way to the Fruition of Perfect Contemplation, and the Rich Treasure of Internal Peace. Written by Dr. Michaelde Molinos, Priest. Translated from the Italian copy, printed at Venice, r685. Edited with an Introduction by Kathleen Lyttelton. And a Note by Canon Scott Holland.

Devotions for Every DAy of the WeEk and the Great Festivals. By John Wesley. Edited, with an Introduction by Canon C. Bodington.

Preces Privatae. By Lancelot Andrewes, Bishop of Winchester. Selections from the Translation by Canon F. E. Brightman. Edited, with an Introduction, by A. E. Burn, D.D.

Horae Mysticae: A Day Book from the Writings of Mystics of Many Nations. Edited by E. C. Gregory.

\section{Little Books on Art}

\section{With many Illustrations. Demy 16mo. 2s. 6d. net.}

Each volume consists of about 200 pages, and contains from 30 to 40 Illustrations, including a Frontispiece in Photogravure.

Albrecht Dürer. J. Allen.

ARTS OP JAPAN, TIIE. E. Dillon.

BOoK Plates. E. Almack.

BotTiCelli. Mary L. Bonnor.

BURNE-JONES. F. de Lisle.

Christ in ART. Mrs. H. Jenner.

Claude. E. Dillon.

Constable, H. W. Tompkins.

CoRot. A. Pollard and E. Birnstingl.

Enamels. Mrs. N. Lawson.

Frederic Leighton. A. Corkran.

GEORge Romney. G. Paston.

GREEK ART. H. B. Walters.

GREUZE AND Boucher. E. F. Pollard

Holbein. Mrs. G. Fortescue.
Illuminated Manuscripts. J. W. Bradley. JEWELLERY. C. Davenport.

JOHN. HOPPNER. H. P. K. Skipton.

Sir Joshua Reynolds. J. Sime.

Millet. N. Peacock.

Miniatures. C. Davenport.

OUR LADY IN ART. Mrs. H. Jenner.

RapHaEl. A. R. Dryhurst. Second Edition.

Rembrandt. Mrs. E. A. Sharp.

TURner. F. Tyrrell-Gill.

VANDYCK. M. G. Smallwood.

Velasquez. W. Wilberforce and A. R. Gilbert.

WATts. R. E. D. Sketchley.

\section{The Little Galleries}

\section{Demy I6mo. 2s. 6d. net.}

Each volume contains zo plates in Photogravure, together with a short outline of the life and work of the master to whom the book is devoted.
A Litrle Gallery of Reynolds.
A LitTle Gallery of Romney.
A Little Gallery of Millais.
A Little Gallery of English Pozts.
A LitTle Gallery of Hoppner. 


\section{Messrs. Methuen's Catalogue}

\section{The Little Guides}

With many Illustrations by E. H. NEw and other artists, and from photographs. Small Pott 8vo, cloth, 2s. 6d. net.; leather, 3s. 6d. net.

The main features of these Guides are (I) a handy and charming form; (2) illustrations from photographs and by well-known artists; (3) good plans and maps ; (4) an adequate but compact presentation of everything that is interesting in the natura. features, history, archæolcgy, and architecture of the town or district treated.

\section{Cambridge and its Colleges. A. H. Hertfordshire. H. W. Tompkins.}

Thompson. Second Edition.

ENGLish Lakes, The. F. G. Brabant.

IsLE of Wighr. The. G. Clinch.

Malvern Country, The B. C. A. Windle.

North Wales. A. T. Story.

OXFORD AND ITS COLleGES. J. Wells. Eighth Edition.

Shakespeare's Country. B. C. A. Windle. Third Edition.

St. Paul's Cathedral. G. Clinch.

Westminster Abiey. G. E. Troutbeck. Second Edition.

Buckinghamshire. E. S. Roscoe.

CHESHIRE. W. MI. Gallichan.

Cornwall. A. L. Salmon.

Derbyshire. J. C. Cox.

Drvon. S. Baring-Gould.

DORSET. F. R. Heath. Second Edition.

Essex. J. C. Cox.

HAMPSHIRE. J. C. Cox.

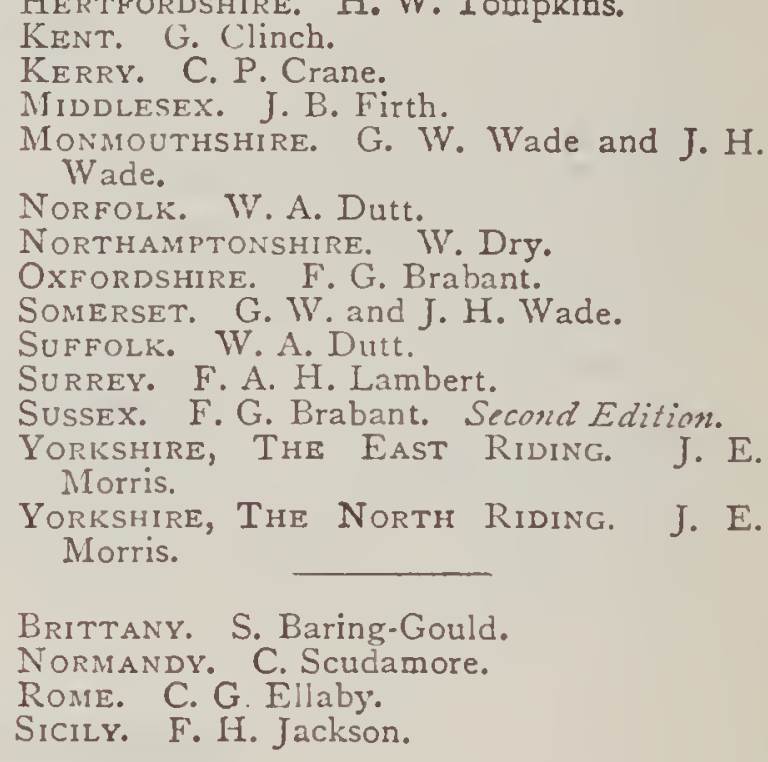

\section{The Little Library}

With Introductions, Notes, and Photogravure Frontispieces.

Small Pott Svo. Each Volume, cloth, Is. 6d. net; leather, 2s. 6d. net. Anon. A LITTLE BOOK OF ENGLISH
LYRICS. Second Edition.

Austen (Jane). PRIDE AND PREJU. DICE. Edited by E. V. Lucas. Truo Vols.

NOR'THANGER ABBEY. Edited by E. V. LUCAS.

Bacon (Francis). THE ESSAYS OF LORD B.ICON. Edited by EDWARD WrIGHT.

Barham (R. H.). THE INGOLDSBY LEGENDS. Edited by J. B. ATLAY. Irwo lolumes.

Barnett (Mrs. P. A.). A LITTLE BOOK OF ENGLISH PIROSE. Sccond Edition. Beckford (IVilliam). THE HISTORY OF THE CALIPH VATHEK. Edited by E. Denison Ross.

Blake (William). SELECTIONS FROM IV I L L A M B LAKE. Edited by M. Peruginl.

Borrow (George). LAVENGRO. Edited by F. HINDES GROONE. Two Volumes.

THE ROMANY RYE. Edited by JOHN SAMPSON.

Browning (Robert). S E L E C T I O N S FROM THE EARIY POEMS OF ROBERT BROWNING. Edited by W. Hall Griffin, M.A. Caxining's additional Poems. Edited by LLOYD SANDERS.

Cowley (Abraham). THE ESSAYS OF ABRAHAM COWLEY. Edited by H. C. MINCHIN.

Crabbe (George). SELECTIONS FROMI GEORGE CRABBE. Edited by A. C. DEANE.

Craik (Mrs.). J OH N H A L I F A X, GENTLE MI N. Edited by ANNIE Matheson. Tivo Volumes.

Crashaw (Richard). THE ENGLISH POEMS OF RICHARD CRASHAW. Edited by EDWARD HuTTON.

Dante (Alighieri). TIHE INFERNO OF DANTE. Translated by H. F. CARY. Edited by PageT 'TOYNBEe, M.A., D.Litt.

THE PURGATORIO OF DANTE. Trans. lated by H. F. CARY. Edited by PAGET TOYNBEE, M.A., D.Litt.

THE PARADISO OF DANTE. Translated by H. F. CARY. Edited by PAGET TorndeE, M.A., D. Litt.

Darley (George). SELECTIONS FROM THE POEMS OF GEORGE DARLEY. Edited by R. A. Streatfeild. 
The Little Library-continued.

Deane (A. C.). A LITTLE BOOK OF LIGHT VERSE.

Dickens (Charles). CHRISTMAS BOOKS. Two Volumes.

Ferrier (Susan). MARRIAGE. Edited by A. GOODRICH - FrEER and LORD IDDESLEIGH. Two Volumes.

THE INHERITANCE. Two Volumes.

Gaskell (Mrs.). CRANFORD. Edited by E. V. LuCas. Second Edition.

Hawthorne (Nathaniel). THE SCARLET LETTER. Edited by PERCy DEARMer.

Henderson ( $T$. F.). A LITTLE BOOK OF SCOTTISH VERSE.

Keats (John). POEMS. With an Introduction by L. Binyon, and Notes by J. MASEFIELD.

Kinglake (A. W.). EOTHEN. With an Introduction and Notes. Second Edition.

Lamb (Charles). ELIA, AND THE LAST ESSAYS OF ELIA. Edited by E. V. LUCAS.

Locker(F.). LONDON LYRICS Edited by A. D. GodLey, M.A. A reprint of the First Edition.

Longfellow (H. W.). S E LE C T I O N S FROM LONGFELLOW. Edited by L. M. F AITHFULL.

Marvell (Andrew). THE POEMS OF ANDREW MARVELL. Edited by $\mathrm{E}$. WRIGHT.

Milton (John). THE MINOR POEMS OF JOHN MILTON. Edited by H. C. BEECHING, M.A.

Moir(D. M.). MANSIE WAUCH. Edited by T. F. HENDERSON.

Nichols (J. B. B.). A LITTLE BOOK OF ENGLISH SONNETS.
Rochefoucauld (La), THE MAXIMS OF LA ROCHEFOUCAULD. Translated by Dean Stanhope. Edited by G. H. POWELl.

Smith (Horace and James). REJECTED ADDRESSES. Edited by A. D. GODLEY, M.A.

Sterne (Laurence). A SENTIMENTAL JOURNEY. Edited by H. W. PAUl.

Tennyson (Alfred, Lord). THE EARLY POEMS OF ALFRED, LORD TENNY. SON. Edited by J. Churton Collins, M.A.

I $N$ M MORIAM. Edited by $H$. C. BEECHING, M.A.

THE PRINCESS. Edited by ElIZABETH WORDSWORTH.

MAUD. Edited by ELIZABETH WORDSWORTH.

Thackeray (W. M.). VAN I T Y FAIR. Edited by S. GwYnN. Three Volumes.

PENDE N N I S. Edited by S. GWynn. Three Volumes.

ESMOND. Edited by S. GwYNN.

CHRISTMAS BOOKS. Edited byS. GWYN.

Vaughan (Henry). THE POEMS OF HENRY VAUGHAN. Edited by EDWARD HuTTON.

Walton (1zaak). THE COMPLEAT ANGLER. Edited by J. BUCHAN.

Waterhouse (Elizabeth). A LITTLE BOOK OF LIFE AND DEATH. Edited by. Twelfth Edition.

Wordsworth (W.). SELECTIONS FROM WORDSWORTH. Edited by NOWELL C. Surth.

Wordsworth (W.) and Coleridge (S. T.). LYRICAL BALLADS. Edited by GEORGE SAMPSON.

\section{The Little Quarto Shakespeare}

Edited by W. J. CRAIG. With Introductions and Notes.

Pott I6mo. In 40 Volumes. Leather, price Is, net each volume.

Mahogany Revolving Book Case. Ios. net.

\section{Miniature Library}

Reprints in miniature of a few interesting books which have qualities of humanity, devotion, or literary genius.

EUPHRANOR: A Dialogue on Youth. By Edward FitzGerald. From the edition published by W. Pickering in 1851 . Denry 32 mo. Leather, 2s. net.

The LIFE OF EDward, LORD HERBERT OF Cherbury. Written by himself. From the edition printed at Strawberry Hill in the year i764. Demy 32mo. Leather, 2s. net.
Polonius: or Wise Saws and Modern Instances. By Edward FitzGerald. From the edition published by IV. Pickering in I852. Demy 32mo. Leather, 2s. net.

The RUbaiy Edward FitzGerald. From the xst edition of 1859 , Fourth Edition. Leather, Is. net. 


\section{A New Historical Series}

Edited by the Rev. H. N. ASMAN, M.A., B.D.

*Stories from Ancient History. By E. * Stories from Modern History. By E. M. Bower, B.A. Cr.8vo. rs. 6d.

\section{The New Library of Medicine}

Edited by C. W. SALEeBY, M.D., F.R.S.Edin. Demy 8vo.

CARE of the Body, ThE. F. Cavanagh. Second Edition. 75. 6\%. net.

Children of The Nation, The. Right Hon. Sir John Gorst. 7s. 6 d. net.

Control of a Scourge, The: or, How Cancer is Curable. Chas. P. Childe. 7s. 6 d. net.

Diseases OF OCCUPATION. Sir Thomas Oliver. ros. 6 \% net.

Drink Problem, The, in its Medico-Sociological Aspects. Edited by T. N. Kelynack. $7 s .6 d . n e t$.
Drugs and the Drug Habit. H. Sainsbury. Functional Nerve Diseases. A. T. Scho. field. $7 s .6$. net.

HYGIENE OF MIND, THE. T. S. Clouston. Fifth Edition. 7s. 6d. net.

INFANT MorTality. George Newman. 7s. 6d. net.

Prevention of Tuberculosis (ConsumpTION), THE. Arthur Newsholme. ros. $6 d$. net.

*Air and Healtu. Ronald C. Macfie, M.A., M.B. $7 s .6 d$. net.

\section{The New Library of Music}

Edited by ERNEST NEWMAN. Demy 8vo. 7s. 6d. net.

Hugo Wolf. By Ernest Newman. With Handel. By R. A. Streatfeild. With iz $r_{3}$ Illustrations.

\section{Oxford Biographies}

Fcap. 8vo. Each volume, cloth, 2s. 6d. net; leather, 3s. 6d. net.

Dante Alighieri. By Paget Toynbee, M.A., Robert Burns. By T. F. Henderson. D.Litt. With I Illustrations. Third Edition. With 12 Illustrations.

Girolamo Savonarola. By E. L. S. Horsburgh, M.A. With Iz Illustrations. Second Edition.

John Howard. By E. C. S. Gibson, D.D., Bishop of Gloucester. With I 2 Illustrations. Alfred Tennyson, By A. C. Benson, M.A. With 9 Illustrations. Second Edition.

Sir Walter Raleigh. By I. A. Taylor. Witli I 2 Illustrations.

Erasmus. By E. F. H. Capey. With I2 Illustrations.

Chatham. By A. S. M'Dowall. With I2 Illustrations.

Francis of Assisi. By Anna M. Stoddart. With r6 Illustrations.

Canning. By IV. Alison Phillips. With 12 Illustrations.

Beaconsfield. By Walter Sichel. With i2 Illustrations.

Johann Wolfgang Goethe. By H. G. Atkins. With r6 Illustrations.

Thi Young Pretender. By C. S. Terry. François Frielon. By Viscount St Cyres.

Witla Iz Illustrations.

With 12 Illustrations.

\section{Romantic History}

\section{Edited by MARTIN HUME, M.A. With Illustrations. Demy 8 vo.}

A series of attractive volumes in which the periods and personalities selected are such as afford romantic human interest, in addition to their historical importance.

The First Governess of the Nether- Hume, M.A. I5s. net.

LANDS, MARgaret of Austria. Eleanor The NiNe Days' QUEEN. Richard Davey.

E. Tremayne. ios. $6 a_{\text {. net. }}$.

Two ENGLish QueEns and Philip Martil

With a Preface by Martin Hume, M.A. With r 2 Illustrations. ros. 6 . net. 


\section{General Literature}

\section{School Examination Series}

Edited by A. M. M. STEDMAN, M.A. Crown 8vo. 2s. $6 d$.

Examination Papers in English Histort. By J. 'Tait Plowden-Wardlaw, B.A.

French Examination Papers. By A. M. M. Stedman, M.A. Fifteenth Edition. KEY. Sixth Edition. 6s. net.

General KNowledge Examination Papers. By A. M. M. Stedman, M.A. Sixth Edition.

KEY. Fourth Edition. 7s. net.

German Examination Papers. By R. J. Morich, Seventh Edition.

KEY. Third Edition. 6s. net.
Greer Examination Papeirs. By A. M. M. Stedman, M.A. Ninth Edition.

$\mathrm{KEY}$, Fourth Edition. 6s. net.

History and Geography Examination PAPERs. By C. H. Spence, M.A. Third Edition.

Latin Examination Papers. By A. M. M. Stedman, M.A. Fourteenth Edition.

KEY. Serienth Edition. 6s. net.

Physics Examination Papers. By R. E. Steel, M.A., F.C.S.

\section{School Histories}

\section{Illustrated. Crown Svo. Is. 6 .}

A School History of Warwickshire. By A School History of Surrey. By $\mathrm{F}^{r}$. E B. C. A. Windle, D.Sc., F.R.S.

A School History of Somerset. By Walter Raymond. Second Edition. Malden, M.A.

Raymond. Second Edition. W. E. Rhodes, M.A. Plarr, M.A., and F. W. Walton, M.A.

\section{Simplified French Texts}

Edited by T. R. N. CROFTS, M.A.

Fcap Svo. Is.

AbDallah. By Edouard Laboulaye. Adapted Le Docteur Mathéus. By Erckmann. by J. A. Wilson.

*Deux Contes. By P. Mérimée. Adapted by J. F. Rhoades.

*Edmond Dantès. By A. Dumas. Adapted by M. Ceppi.

Jean Valjean. By Victor Hugo. Adapted by F. IV. M. Draper, M.A.

La Bataille de Waterloo. By Erckmann. Chatrian. Adapted by G. H. Evans.

La Bouillie au Miel. By A. Dumas. Adapted by P. B. Ingham, M.A.

La Chanson DE Roland. Adapted by $\mathrm{H}$. Rieu, M.A. Second Edition.

LE ConsCRIT DE I8I3. By Erckmann-Chatrian. Adapted by H. Rieu.

Chatrian. Adapted by W. P. Fuller, M.A.

* Le Duc de Beaufort. By A. Dumas. Adapted by P. B. Ingham, M.A.

L'EQUIPAGE DE LA BELLE-Nivernaise. By Alphonse Daudet. Adapted by T. R. N. Crofts, M.A.

L'Histoire D'une, Tulire. By A. Dumas. Adapted by T. R. N. Crofts, MI.A. Second Edition.

L'Histoire de Pierre et Camille. BÿA. de Musset. Adapted by J. B. Patterson, M.A.

MÉnoIREs DE CADICHON. By Madam de Ségur. Adapted by J. F. Rhoades.

* D'A jaccio A Saint Helène. By A. Dumas. Adapted by F. IV. M. Draper, M.A.

Remy LE CHevrier. By I.. Souvestre. Adapted by E. E. Chottin, B-es-L.

\section{Simplified German Texts}

Edited by T. R. G. CROFTS, M. A. Fcap. 8vo. Is.

Der Muller am Rhein. By C. Brentano. Die Notheifer. By. W. H. Riehl. Adapted Adapted by Florence A. Ryan.

Die Geschichte von Peter Schlemihl. ByA.v. Chamisso. Adapted by R. C. Perry. by P. B. Ingham, M.A.

Undine und HuldBRAND. By La Motte Fouqué. Adapted by T. R. N. Crofts, M.A 


\section{Six Ages of European History}

Edited by A. H. JOHNSON, M.A. With Maps. Crown 8vo. 2s. 6d.

Age of THE ENLightented Despot, THE, I660-1789. A. H. Johnson.

Central Period of the Middle Age, the, 918-1273. Beatrice A. Lees.

Dawn of MEdiaval Europe, The, 476-9i8. J. H. B. Masterman.
END OF THE MIIDDLE AGE, THE, 1273.1453. E. C. Lodge.

Europe in Renaissance and ReformaTION, I453-I659. M. A. Hollings.

REMAKING OF MODERN EuROPE, THE, 1789 I878. J. A. R. Marriott.

\section{Methuen's Standard Library}

Cloth, Is. net; doublevolumes, Is. 6 d.net. Paper, $6 d$. net; double volume, Is. net.

The Meditations of Marcus Aurelius. 'Translated by R. Graves.

Sense and Sensibility. Jane Austen.

ESSAYS AND COUNSEls and The NEw Atrantis. Francis Bacon, Lord Verulam.

Religio MIEDICI and URN BURIal. Sir Thomas Browne. The text collated by A. R. Waller.

The Pilgrim's Progress. John Bunyan.

Reflections on the French Revolution. Edmund Burke.

The Poems and Songs of Robert Burns. Double Volume.

The Analogy of Religion, Natural and Revealed. Joseph Butler.

Miscellaneous Poems. T. Chatterton.

The Rowley Poems. T. Chatterton.

Tom Jones. Henry Fielding. Treble Vol.

Cranpord. Mrs. Gaskell.

The Poemsand Plays of Oliver Goldsmith.

The Case is Altered. Fvery Man in His Humour. Every Man Out of His Humour. Ben Jonson.

Cynthia's Revels. Poetaster. Ben Jonson.
The Poems of John Keats. Double volume. The Text has been collated by E. de Sélincourt.

On the Imitation of Christ. By Thomas à Kempis. Translation by C. Bigg.

A Serious Call to a Devout and Holy LIFE. W. Law.

Paradise Lost. John Milton.

Eikonoklastes and the TrinuRe of King and Magistrates. John Milton.

Utopia and Poems. Sir Thomas More.

The Republic of Plato. Translated by Sydenham and Taylor. Double Volume. Translation revised by W. H. D. Rouse.

The Little Flowers of St. Francis. Translated by W. Heywood.

The Works of William Shakespeare. In Io volumes.

The Poems of Percy Bysshe Shelley. In 4 volumes. With Introductions by C. D. Locock.

The LifE of Nelson. Robert Southey.

Tiie Natural Historyand Antiquities of SELBORNE. Gilbert White.

\section{Textbooks of Science}

Edited by G. F. GOODCHILD, M.A., B.Sc., and G. R. MILLS, M.A.

\section{Fully Illustrated.}

Complete School Chemistry, The. By F. I. Oldham, B.A. With I 66 llustrations. Thard Edition. Cr. 8vo. 4s. 6 d.

Eleaientary Science for Pupil Teachers. Physics Section. By W. T. Clough, A.R.C.Sc. (Lond.), F.C.S. Chemistry Section. ByA. E. Dunstan, B.Sc. (Lond.), F.C.S. With 2 Plates and ro Diagrams. Cr. 8ro. 2s.

Examples in Elementary Mechanics, Practical, Graphical, and Theoretical. By W. J. Doubs, M.A. With 52 Diagrams. Cr. 8\%o. 55 .

Examples in Puysics. By C. E. Jackson, M.A. Cr. 8vo. 2s. $6 d$.

First Year Physics. By C. E. Jackson, M.A. With 5I Diagrams. Cr. 8vo. is. $6 d$.

Outlines of Physical Chemistry. George Senter, B.Sc. (Lond.), Ph. D. With many Diagrams. Cr. $800.35 .6 \%$.
ORganic Chemistry, AN, FOR SChOOLS AND 'Technical Institutes. By A. E. Dunstan, B.Sc. (Lond.), F.C.S. With many Hllustrations. Cr. 8\%o. 25. $6 d$.

Plant Life, Studies in Garden and School. By Horace F. Jones, F.C.S. With 320 Illustrations. Cr. 8vo. 3s. 6 \%

Practical Chemistry. Part I. W. French, II.A. Fifth Edition. Cr. 8vo. Is. 6 d.

Practical Chemistry. Part il. W. French, M.A., and T. H. Boardman, M.A. Cr. Szo. is. 6 .

* Practical Chemistry for Schools and 'Technical Institutes, A. By A. E. Dunstan, B.Sc. (Lond.), F.C.S. Cr. 8zo. 3s. $6 d$. Practical Mechanics. S. H. Wells. Fourth Edition. Cr. 8vo. 3s. 6d.

Technical Arithmetic and Geometry. By C. T. Millis, M.I.M.E. Cr. Suo. 3s. $6 d$. 


\title{
General Literature
}

\section{Textbooks of Technology}

\author{
Fully Illustrated.
}

Builders' Quantities. By H. C. Grubb. How to Make a Dress. By J. A. E. Wood. Cr. 8v0. 4s. 6d.

Carpentry and Joinery. By F. C. Webber. Fifth Edition. Cr. 8vo. 3s. 6 d.

Electicic Light and Power: An Intro. duction to the Study of Electrical Engineering. By E. E. Brooks, B.Sc. (Lond.). and W. H. N. James, A.M.I.E.E., A.R.C.Sc. Cr. 8vo. 4s. 6d.

Engineering Workshop Practice. By C. C. Allen. Cr. 8vo. 3s. $6 d$. Fourth Edition. Cr. 8vo. Is. 6 .

INSTRUCTION IN COOKERY. A. P. ThOMSON. Cr. 8vo. 2s. $6 d$.

Introduction to thr Study of Textile Design, An. By Aldred F. Barker. Demy 8vo. $75.6 d$.

Millinery, Theoretical and Practical. By Clare Hill. Fourth Edition. Cr.8vo. 2s

RÉPOUSSÉ METAL IVORK. By A. C. Horth. Cr. 8vo. 2s. 6 .

\section{Handbooks of Theology}

The Doctrine of the Incarnation. By R. AN Introduction to the History of the L. Ottley, D.D. Fourth Edition revised. Demy 8vo. I2s. 6d.

A History of Eariy Christian Doctrinf. By J. F. Bethune-Baker, M.A. Demy 8 vo. IOS. $6 d$.

AN INTRODUCTION to THE History OF RELigion. By F. B. Jevons. M.A., Litt. D. Fourth Edition. Demy 8vo. ros. 6 d. Creeds. By A. E. Burn; D.D. Demy 8vo. 10s. 6d.

The Philosophy of Religion in England And America. By Alfred Caldecott, D.D. Demy 8vo. Ios. 6d.

THE XXXIX. ARTICLES OF THE CHURCH OF ENGLAND. Edited by E. C. S. Gibson, D.D. Sixth Edition. Demy 8vo. 12s.6d.

\section{The Westminster Commentaries}

General Editor, WALTER LOCK, D.D., Warden of Keble College, Dean Ireland's Professor of Exegesis in the University of Oxford.

The Acts of the Aposties. Edited by R. The Book of Ezekiel. Edited H. A. Red. B. Rackham, M.A. Demy 8vo. Fourth Edition. Ios. 6 d.

The First Epistle of Paul the Apostie TO THE Corinthians. Edited by H. L. Goudge, M.A. Second Ed. Demy8vo. 6s.

A Commentary on Exodus. By A. H. M'Neile, B.D. With a Map and 3 Plans. Demy 8vo. Ios. 6 d. path, M.A. D. Litt. Denry 8vo. Ios, $6 d$.

The BOOK of Genesis. Edited with Introduction and Notes by S. R. Driver, D.D. Seventh Eclition Demy 8vo. ros. 6d.

THE Book of Joe. Edited by E. C. S. Gibson, D.D. Second Edition. Demy 8ro. 6s.

The EPIstle of St. James. Edited with In. troduction and Notes by R. J. Knowling, D.D. Demy 8vo. 6s.

\section{Part II.—Fiction}

Albanesi (E. Maria). SUSANNAH AND THE INVINCIBLE AMELIA: or, THI

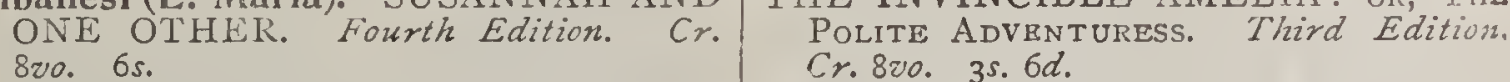
8vo. $6 s$.

THE BLUNDER OF AN INNOCENT. SecondEdition. Cr. 8vo. 6 s.

CAPRICIOUS CAROLINE. Second Edi. tion. Cr. 8vo. 6s.

LOVE AND LOUISA. Second Edition. Cr. 8vo. 6s. Also Medium 8vo. 6d.

PETER, A PARASITE. Cr. 8vo. 6s.

THE BROWN EYES OF MARY. Third Edition. Cr. 8vo. 6s.

I KNOW A MAIDEN. Third Edition. Cr. 8vo. 6s. Also Medium 8vo. 6d.

Annesley (Maude). THIS DAY'S MADNESS. Cr.8vo. $6 s$.

Anstey (F.). A BAYARD FROM BENGAL. Medium 8vo. $6 d$.

Austen (Jane). PRIDE AND PREJU. DICE. Medium8vo. 6d.

Aveling (Francis). ARNOUL THE ENGLISHMAN. Cr. 8vo. $6 s$.

Bagot(Richard). A ROMAN MYSTERY. Third Edition. Cr. 8ro. 6s.

Also Medium 8vo. $6 d$. 
THE PASSPORT. Fourth Edition. Cr. 8v0. 6s.

TEMPTATION. Fifth Edition. Cr. $8 \pi 0$. $6 s$.

ANTHONY CUTHEERT. Fourth Edition Cr. 8\%o. 6s.

LOVE'S PROXY. A Neru Edition. Cr. 8vo. $6 s$.

DONNA DIANA. Second Edition. Cr. 8vo. 6s. Also IIedium 8vo. 6d.

CASTING OF NETS. Twelfth Edition. Cr. 8vo. 6s. Also Medium 8vo. 6d.

Balfour (Andrew). BY STROKE OF SIVORD. Mredium sio. $6 d$.

Ball (Oona H.) (Barbara Burke). THEIR OXFORD YEAR. With 16 Illustrations Cr. 8vo. 6s.

BARBARA GOES TO OXFORD. With i 6 Illustrations. Third Edition. Cr. 8vo. 6s.

Baring-Gould (S.). ARMINELL. Fifth Edition. Cr. 8vio. 6s.

Also Mediun 8vo. 6 .

URITH. Fifth Eilition. Cr. 8vo. 6s. Also Medium 8vo. $6 d$.

IN THE ROAR OF THE SEA. Sezenth Edition. Cr. 8vo. 6s.

Also Medium 8vo. $6 d$.

CHEAP JACK ZITA. Medium 8io. $6 d$.

MARGERY OF QUETHER. Third Edition. Cr.8\%o. 6s.

THE QUEEN OF LOVE. Fifth Fitition. Cr. 8vo. 6s. Also Medium 8vo. 6i.

JACQUETTA. Third Edition. Cr.8vo. 6s.

KITTY ALONE. Fifth Erition. Cr. 8vo. 6s。 Also Medium 8vo. $6 d$.

NOÉMI. Illustrated. Fourth Edition. Cr. 8vo. 6s. Also Medium 8vo. 6r.

THE BROOM-SQUIRE. Illustrated. Fifth Edition. Cr. 8vo. 6s.

Also Medium 8ro. $6 \%$.

DARTMOOR IDYLLS. Cr. 8vo. $6 s$.

GUAVAS TIE TINNER. Illustrated. Second Edition. Cr. 8vo. 6s.

BLADYS OF THE STEWPONEY. Illustrated. Second Eidition. Cr. 8vo. 6s.

PABO THE PRIEST. Cr. 8vo. 6s.

IVINEFRED. Illustrated. Srcond lidition. Cr. 8vo. 6s. Also Medium 8\%o. 6\%.

ROYAL GEORGIE. Illustrated. Cr. 8vo. 6 s.

CHRIS OF ALL SORTS. Cr. 8z'o. 6s.

IN DEWISLAND. Second Ed. Cr. 8vo. 6s. THE FROBISHIES. Cromin 8vo. $6 \mathrm{~s}$. Also Medium 8vo. 6 \%.

DOMITIA. Illus. Second Ed. Cr. 8vo. 6s. MRS. CURGENVIEN OF CURGENVIEN. Croiun 8vo. $6 s$.

LITTLE TU'PENNY. Medium 8\%o. $6 \Omega$.

FURZE BLOOM. Mediums:o. 6\%.

Barnett (Edith A.). A WILDISRNESS WINNER. Second Edition. Cr. 8vo. Fs.
Barr (James). IAUGHING THROUGH A WILDERNESS. Cr. 870. $6 s$.

Barr (Robert). IN THE MIDST OF ALARMS. Third Edition. Cr. 8vo. 6s. Also Medium 8vo. $6 d$.

THE COUNTISS TEKLA. Fourth Edition. Cr. 8vo. 6s.

Also Medium 8vo. $6 d$.

THE MUTABLE MANY. Third Edition. Cr. 8vo. 6s. Also Medium 8vo. 6d.

THE TEMPESTUOUS PETTICOAT. Illustrated. Third Edition. Cr. 8vo. $6 s$. JENNIE BAXTER JOURNALIST. Medium 8vo. 6d.

Begbie (Harold). THE CURIOUS AND DIVERTING ADVENTURES OF SIR JOHN SPARROW; or, THE Progress OF AN OPEN MIND. With a Frontispiece. Second Edition. Cr.8vo. 6s.

Belloc (H.), EMMANUEL BURDEN, MERCHANT. With 36 Illustrations by G.K.Chesterton. Second Fid. Cr. 8vo. 6s.

A CHANGE IN THE CABINET. Second Edition. Cr. 8vo. 6s.

Benson (E. F.) DODO : A DETAIL OF THE DAY. Fificenth Edition. Cr. 8z'o. 6s. Also Medium 8vo. $6 \%$.

THE VINTAGE. Medium 8\%o. $6 d$.

Benson (Margaret). SUBJECT TO VANITY. Cr. 8vo. 3s. 6d.

Birmingham (George A.). THE BAD TIMES. Second Edition. Cr. 8jo. 6s.

SPANISH GOLD. Fourth Edition. Cr. $8 \approx 0.65$.

THE SEARCH PARTY. Cr. 8vo. 6s.

Bowles (G. Stewart). A GUN-ROOM DITTY BOX. Second Ed. Cr. 8vo. Is.6d.

Bretherton (Ralph Harold). TH E MILL. Cr. 8vo. 6s.

AN HONEST MAN. Second Edtition. Cr. 8i'o. 6s.

Brontë (Charlotte). SHIRLEY. Medium 8vo. $6 d$.

Burton (J. Bloundelle). ACROSS THE SALT SEAS. Wecium 8vo. 6\%.

Caffyn (Mrs.) ('lota'). ANNE MAULEVERER. Medium 8vo. $6 d$.

Campbell (Mrs. Vere). F E R R I Y. Second Exition. Cr. 8*'o. 6s.

Capes (Bernard). THE FXTRAORDINARY CONFESSIONS OF DIANA PLEASE. Third Edition. Cr. 8vo. $6 s$. A JAY OF ITALY. Fourth Ea. Cr. 8vo. 6s. LOAVES AND FISHES. Second Edition. Cr. 8zo. 6s.

A ROGUE'S TRAGEDY. Second Edition. Cr. 8vo. 6s.

THE GREAT SKENE MYSTERY. Second liditios. Cr. 8vo. 6s.

TIIE LOVE STORY OF ST. BEL. Second Edition. Cr. 8vo. 6s. 
THE LAKE OF WINE. Medium S $\sim$. $6 d$. Carey (Wymond). LOVE THE JUDGE. Second Edition. Cr.8vo. 6s.

Castle (Agnes and Egerton). FLOWER $O^{\prime}$ THE ORANGE, and Other Tales. With a Frontispiece in Colour by A. H. Buckland. Third Edition. Cr. 8vo. 6s.

Charlton (Randal). M A V E. Second Edition. Cr. 8vo. 6s.

THE VIRGIN WIDOW. Cr. 8vo. $6 s$.

Chesney (Weatherby). THE MYSTERY OF A BUNGALOW. Second Edition. Cr. 8vo. 6s.

Clifford (Mrs. W. K.). THE GETTING IVELL OF DOROTHY. Illustrated by Gordon Browne. Second Edition. Cr. 8vo. $35.6 d$.

A FLASH OF SUMMER. Medium 8vo. $6 d^{2}$. MRS. KEITH'S CRIME. Medium 8vo. 6a'.

Conrad(Joseph). THE SECRET AGENT: A Simple Tale. Fourth Ed. Cr. 8vo. 6s.

A SET OF SIX. Fourth Edition. Cr. 8vo. $6 s$.

Corbett (Julian). A BUSINESS IN GREAT WATERS. Third Edition. Cr. 8vo. 6s. Also Mediun 8vo. 6d.

Corelli (Marie). A ROMANCE OF TWO WORLDS. Twenty-Ninth Ed. Cr.8vo. $6 \mathrm{~s}$.

VENDETTA. Twenty-Seventh Edition. $C \%$ 8vo. 6s.

THELMA. Thirty-Nint/2 Ed. Cr. 8vo. 6s. ARDATH : THE STORY OF A DEAD SELF. Nineteenth Edition. Cr. 8vo. 6s. THE SOUL OF LILITH. Sixteenth $E d i$ tion. Cr. 8vo. 6s.

WORMWOOD. SixteenthEd. Cr.8ro. 6s.

BARABBAS: A DREAM OF THI WORLD'S TRAGEDY. Forty-Fourth Edition. Cr. 8ro. 6s.

THE SORROWS OF SATAN. Fifty-Fifth Edition. Cr. 8vo. 6s.

THE MASTER CHRISTIAN. Twelfth Edition. I77th Thousand. Cr.8vo. 6s.

TEMPORAL POWER: A STUDY IN SUPREMACY. Second Edition. I50th Thousand. Cr. 8vo. 6s.

GOD'S GOOD MAN : A SIMPLE LOVI STORY. Thirteenth Edition. I50th Thousand. Cr. 8vo. $6 s$.

HOLY ORDERS: THE TRAGEDY OF A QUiET LIFE. Second Edition. I2oth Thousand. Croun 8vo. 6s.

THE MIGHTY ATOM. Twenty-seventh Edition. Cr. 8vo. 6s.

BOY : a Sketch. Eleventh Edition. Cr. 8vo. $6 s$.

CAMEOS. Thirteenth Edition. Cr. 8vo. 6s.

Cotes (Mrs. Everard). See Duncan (Sara Jeannette).

Cotterell (Constance). THE VIRGIN AND THE SCALES. Illustrated. Second Edition. Cr. 8vo. $6 s$.
Crockett (S. R.), LOCHINVAR. Illus. trated. Third Edition. Cr.8vo. 6s. $A$ iso Medium 8vo. $6 d$.

THE STANDARD BEARER. Cr. 8vo. $6 s$.

Croker (Mrs. B. M.). THE OLD CAN. TONMENT. Cr.8vo. $6 s$.

JOHANNA. Second Edition. Cr.8vo. $6 s$. Also Medium Svo. 6d.

THE HAPPY VALLEY. Fourth Edition. Cr. 8vo. 6s.

A NINE DAYS' WONDER. Third Edition. Cr. 8vo. 6s.

PEGGY OF THE BARTONS. Seventin Ed. Cr. 8vo. 6s. Also Medium 8vo. 6\%.

ANGEL. Fifth Eatition. Cr. 8vo. 6s. Also Medium 8vo. $6 d$.

A STATE SECRET. Third Edition. Cr. 8vo. 3s. 6d. Also Mediun 8vo. 6d.

KATHERINE THE ARROGANT. Fifth Edition. Cr. 8vo. 6s.

Crosbie (Mary). DISCIPLES. Second Ed. Cr. 8vo. 6s.

Cuthell (Edith E.). ONLY A GUARD. ROOM DOG. Illustrated by W. PARKIN son. Crown 8vo. 3s. 6 .

Dawson (Warrington). THE SCAR. Second Edition. Cr. 8vo. 6s.

THE SCOURGE Cr.8vo. $6 s$.

Deakin (Dorothea). THE YOUNG COLUMBINE. With a Frontispiece by Lewis Baumer. Cr. 8vo. $6 s$.

Deane (Mary). THE OTHER PAWN. Cr. 8vo. 6s.

Doyle (A. Conan). ROUND THE RED LAMP. Eleventh Edition. Cr. 8vo. 6s. Also Mtedium 8vo. $6 d$.

Dumas (Alexandre). See page ${ }_{4} 6$.

Duncan (Sara Jeannette) (Mrs. Everard Cotes). THOSE DELIGH T F U AMERICANS. Medium 8vo. $6 d^{\circ}$

A VOYAGE OF CONSOLATION. Illustrated. Third Edition. Cr. 8vo. 6s. Also Medium 8vo. 6d.

COUSIN CINDERELLA. Second Edition. Cr. 8vo. 6s.

THE BURNT OFFERING. Cr. 8vo. $6 s$.

Eldridge(George D.). IN THE POTTER'S HOUSE. $C 3.870$. $6 s$.

Eliot (George). THE MILL ON THE FLOSS. Medium 8vo. $6 d$.

Erskine (Mrs. Steuart). THE MAGIC PLUMES. Cr. 8vo. $6 s$.

Fenn (G. Manville). SYD BELTON ; or, The Boy who would not go to Sea. Illustrated by Gordon Browne. Second Ed. Cr. 8vo. 3s. 6 \%

Findlater (J.H.). THE GREEN GRAVES OF BALGOWRIE. Fift/ Edition. Cr. 8vo. 6s. Also Medium 8vo. 6d.

THE LADDER TO THE STARS. Secord Edition. Cr. 8vo. $6 \mathrm{~s}$. 
Findlater (Mary). A NARROW WAY. Third Edition. Cr. 8vo. 6s.

OVER THE HILLS. Second Edition. Cr. 8vo. $6 s$.

THE ROSE OF JOY. Third Edition. Cr. 8vo. 6s.

A BLIND BIRD'S NEST. With 8 Illus. trations. Sccond Edition. Cr. 8vo. 6s.

Fitzpatrick (K.) THE WEANS AT RUWALLAN. Illustrated. Second Edition. Cr. 8vo. $6 s$.

Francis (M. E.). (Mrs. Francis Blundell). STEPPING WESTWARD. Second Edition. Cr. 8vo. 6 s.

MARGERY O' THE MILL. Third Edition. Cr. 8vo. 6s.

HARDY-ON-THE-HILL. Third Edition. Cr. 8vo. 6s.

GALATEA OF THE WHEATFIELD. Sccona'Edition. Cr.8vo. 6s.

Fraser (Mrs. Hugh). THE SLAKING OF THE SWORD. Second Edition. Cr. 8vo. 6s.

GIANNELIA. Cr. 8vo. 6s.

IN THE SHADOW OF THE LORD. Third Edition. Croun 8vo. $6 s$.

Fry (B. and C.B.). A MOTHER'S SON. Fifth Ecition. Cr. 8vo. 6s.

Fuller-Maitland (Ella). B L N C H E ESMEAD. Second Edition. Cr. 8ro. 6s.

Gallon (Tom). RICKERBY'S FOLLY. Medium 8vo. $6 d$.

Gaskell (Mrs.). CRANFORD. Medium 8vo. $6 \pi$.

MARY BARTON. Mediumsvo. $6 d$.

NORTH AND SOUTH. Medium 8vo. $6 a$. Gates(Eleanor). THE PLOW-WOMAN. Cr. 8vo. 6s.

Gerard (Dorothea). HOLY MATRIMONY. Medium 8vo. $6 d$.

MADE OF MONEY. Medium 8zo. $6 d$.

THE INPROBABLE IDYL. Third Edition. Cr. 8vo. 6s.

THE BRIDGE OF LIFE. Cr. 8\%o. $6 s$.

THE CONQUEST OF LONDON. Mealium 8vo. $6 d$.

Gibbs (Philip). THE SPIRIT OF REVOLT. Second Edition. Cr. 8vo. 6s.

Gissing (George). THE TOWN TRA. VLLLLR. Medium 8zo. $6 d$.

THE CROWN OF LIFE. Cr. 8vo. $6 s$. Also Medium 8vo. $6 d$.

Glanville (Ernest). THE INCA'S TREA. SURE. Illustrated. Cr. 8vo. $35.6 d^{\circ}$. Also Medium 8vo. $6 d$.

THE KLOOF BRIDE. Medium 8vo. $6 d$. Gleig (Charles). BUNTER'S CRUISE. Illustrated. Cr. 8vo. $35.6 d$. Also Medium 8vo. $6 d$.

Grimm (The Brothers). GRIMM'S FAIRY TALES. Illustrated. Medism 8vo. 6 .
Haig (J. C.). IN THE GRIP UF THE TRUSTS: A STORY OF IgI4. Cr. 8vo. Is. net.

Hamliton (M.). THE FIRST CLAIM. Second Edition. Cr.8vo. 6s.

Harraden (Beatrice). I N VARYING MOODS. Fourteenth Edition. Cr.8vo. 6s. THE SCHOLAR'S DAUGHTER. Fourth Edition. Cr. 8vo. 6s.

HILDA STRAFFORD and THE REMIT. TANCE IIAN. Twelfth Ed. Cr. 8vo. $6 s$.

INTERPLAY. Fifth Edition. Cr. 8vo. 6s.

Harrod (F.) (Frances Forbes Robertson). THE TAMING OF THE BRUTE. Cr. 8vo. 6s.

Hart (Mabel). SISTER K. Cr. 8vo. $6 s$.

Hichens (Robert). THE PROPHET OF BERKELEY SQUARE. Second Edition. Cr. 8vo. 6s.

TONGUES OF CONSCIENCE. Third Edition. Cr. 8vo. 6s.

FELIX. Sixth Edition. Cr. 8vo. 6s.

THE WOMAN WITH THE FAN. Serenth Edition. Cr. 8vo. 6s.

BYEWAYS. Cr. 8ro. $6 s$.

THE GARDEN OF ALLAH. Eighteenth Edition. Cr. 8vo. 6 s.

THE BLACK SPANIEL. Cr. 8vo. 6s.

THE CALL OF THE BLOOD. Seventh Edition. Cr.8vo. 6s.

BARBARY SHEEP. Second Edition. Cr. 8vo. 3s. 6d.

Hope (Anthony). THE GOD IN THE CAR. Eleventh Edition. Cr.8vo. 6s.

A CHANGE OF AIR. Sixth Ed. Cr.8vo. 6s. Also Medium 8vo. 6d.

A MAN OF MARK. Sixth Ed. Cr. 8vo. 6s Also Medium 8vo. 6d.

THE CHRONICLES OF COUNT AN. TONIO. Sixth Edition. Cr. 8vo. 6s. Also Medium 8vo. 6d.

PHROSO. Illustrated by H. R. MILLAK. Eighth Edition. Cr. 8vo. 6s. Also Medium 8vo. $6 d$.

SIMON DALE. Illustrated. Eight/2 Edition. Cr. 8vo. 6s.

THE KING'S MIRROR. Fourth Edition. Cr. 8vo. $6 s$.

QUISANTE. Fourth Edition. Cr. 8vo. 6s. THE DOLLY DIALOGUES. Cr. 8vo. $6 \mathrm{~s}$. Also Mcdiun 8vo. 6a.

A SERVANT OF THE PUBLIC. Illustrated. Fourth Edition. Cr. 8vo. 6s.

TALES OF TWO PEOPLE. With a Frontispiece by A. H. BuckLAND. Third Ed. Cr. 8vo. 6s.

THE GREAT MISS DRIVER. With a Frontispiece by A. H. BUCKLAND. Fourt/ Edition. Cr. 8vo. 6s. 
Hornung (E. W.). DEAD MEN TELL NO TALES. Medium8vo. 6d.

Housman (Clemence). THE LIFE OF SIR AGLOVALE DE GALIS. Cr. $8 \pi \circ .6 s$.

Hueffer (Ford Madox). AN FNGIJSH GIRL: A Romance. Second Fidition. Cr. 8ro. $6 s$.

MR. APOLLO: A Just Possible Story. Second Edition. Cr. 8io. $6 s$.

Hutten(Baroness von). THE HALO. Fifth Edition. Cr. 8vo. 6s.

Hyne (C. J. Cutcliffe). MR. HOR. ROCKS, PURSER. Fourth Edition. Cr. 8vo. 6s.

PRINCE RUPERT, THE BUCCANEER. Illustrated. Third Edition. Cr. 8zo. 6s.

Ingraham (J. H.). THE THRONE OI' DAVID. Medium 8vo. $6 d$.

Jacobs (W. W.). MANY CARGOES. Thirty-first Edition. Cr. 8*o. 35.6 .

SEA URCHINS. Fifteenth Edition. Cr. 8vo. $3 s .6$.

A MASTER OF CRAFT. Illustrated by WILI OWEN. Eighth Edition. Cr. 8थ0. $35.6 \%$.

LIGHT FREIGHTS. Illustrated by WILI OWEN and Others. Sezcnth Eidition. Cr. $800.35 .6 d$.

THE SKIPPER'S WOOING. Ninth Fdition. Cr. 8vo. $3 s .6 \%$.

AT SUNWICH PORT. Illustrated by WILl OWEN. Ninth Edition. Cr.8wo. 3s.6\%.

DIALSTONE LANE. Illustrated by WILL OWEn. Seventh Edition. Cr. 8vo. $3 s .6 \%$.

ODD CRAFT. Illustrated by WILL OWEN. Third Edition. Cr. 8vo. $3^{s .6}$.

THE LADY OF THE BARGE. Illustrated. Eighth Edition. Cr. 8vo. $3^{5.6}$.

SALTHAVEN. Illustrated by WiLl OWEN. Second Edition. Cr. 8\%o. 3 s. 6d.

SAILORS' KNOTS. Cr. 8vo. 3s. $6 d$.

James (Henry). THE SOFT SIDE. Second Edition. Cr. 8vo. $6 s$.

THE BETTER SORT. Cr. 8\%o. $6 s$.

THE GOLDEN BOWL. Third Edition. Cr. 8vo. 6s.

Keays (H. A. Mitchell). HE THAT EATETH BREAD WITH ME. $C r$. 8vo. $6 s$.

Kester(Vaughan). THE FORTUNES OI THE LANDRAYS. Cr. 8vo. $6 s$.

Lawless (Hon. Emily). WITH ESSEX IN IRELAND. Cr. 8vo. $6 s$.

Le Queux (William). THE HUNCHBACK OF WESTMINSTER. Third Ed. Cr. 8vo. 6s. Also Medium 8vo. 6d.

THE CLOSED BOOK. Third Edition. Cr. 8vo. 6s.

THE VALLEY OF THE SHADOW Illustrated. Third Edition. Cr. 8vo. $6 s$.

BEHIND THE THRONE. Third Edition. Cr. 8vo. 6s.
THE CROOKED WAY. Second Edition. Cr. 8\%o. 65 .

Levett $=$ Yeats $(S . K$.$) ORRAIN. Second$ Edition. Cr. 8\%o. 6s. Also Medium $82 \% .6 \%$.

THE TRAITOR'S WAY. Nedium 8vo. 6d.

Linton(E. Lynn). THE TRUE HISTORY OF JOSHUA DAVIDSON. Medium $8 \pi 0.6 \%$.

London (Jack). WHITE FANG. With a Frontispiece by CHARLES RIVINGSTON Bull. Sixth Edition. Cr. 8vo. 6s.

Lubbock (Basil). DIEP SEA WAR. RIORS. Illustrated. Second Fidition. Cr. $8 \approx 0.6 s$.

*Lucas (St. John). THF, FIRST ROUND. Cr. 8vo. 6s.

Lyall (Edna). DERRICK VAUGHAN, NOVIELIST. 43rd Thousand. Cr. 8vo 3s. $6 d$. Also Medium 8ro. $6 d$.

Maartens (Maarten). THE NITW RELI. GION: A MODlikN Novel. Third Edi. tion. Cr. 8ro. $6 s$.

PROTHERS ALL; MORE STORIES OF Dutcir Peasan' LifF. Third Edition. Cr. 8vo. 6s.

'THE PRICE OF LIS 1)ORIS. Cr. $8{ }^{\circ} 0.6 s$.

M'Carthy (Justin H.). THE LADY OF LOYALTY HOUSE. Illustrated. T\%ird Edition. Cr. 8vo. 6s.

THE DRYAD. Second Edition. Cr. 8vo. 6s. THE DUKE'S MOT'TO. Third Edition. Cr. 8vo. 6s.

Macdonald (Ronald). A HUMAN TRINITY. Second Edition, Cr. 8vo. 6s.

Macnaughtan (S.). THE FORTUNE OF CHRISTINA M'NAB. Fourth Edition. Cr. 8vo. 6s.

Malet (Lucas). COLONEL ENDEREY'S WIFE. Fourth Edition. Cr. 8vo. 6s.

A COUNSEL OF PERFECTION. Second Edition. Cr. 8vo. $6 s$.

Also Mediwm 8vo. $6 d$.

THE WAGES OF SIN. Sirtenth Edition. Cr. 8vo. 6s.

THE CARISSIMA. Fifth Ed. Cr. 8vo. Gs. Also Medium 8vo. $6 d$.

THE GATELESS BARRIER. Fifth Edition. Cr. 8vo. 6s.

THE HISTORY OF SIR RICHARD CALMADY. Seventh Edition. Cr.8vo. 6s.

Mann (Mrs. M. E.). OLIVIA'S SUMMER. Second Edition. Cr. 8vo. 6s.

A LOST ESTATE. A New Ed. Cr. 8vo. 6s. Also Medium 8vo. $6 d$.

THE PARISH OF HILBY. A New Edition. Cr. 8vo. 65.

THE PARISH NURSE. Fourth Edition. Cr. 8vo. 6s.

GRAN'MA'S JANE. Cr.8vo. $6 s$. 
MRS. PETER HOWARD. Second Edition. Cr. 8vo. 6s. Also Medium 8vo. 6d.

A WINTER'S TALE. A Nere Edition. Cr. 8vo. 6s. Also Medium 8vo. 6r.

ONE ANOTHER'S BURDENS. A New Edition. Cr.8vo. $6 s$.

Also Medium 8vo. $6 \pi$.

ROSE AT HONEYPOT. Third Ed. Cr. 8vo. 6s.

THERE WAS ONCE A PRINCE. Illustrated by M. B. MANN. Cr. 8vo. 3s. $6 d$.

WHEN ARNOLD COMES HOME. Illustrated by M. B. MaNn. Cr. 8vo. 3s. $6 d$.

THE EGLAMORE PORTRAITS. Tiir d Edition. Cr.8vo. 6s.

THE MEMORIES OF RONALD LOVE. Cr. 8vo. 6s.

THESHEEP AND THE GOATS. Third Edition. Cr. 8vo. 6s.

A SHEAF OF CORN. Second Edition. Cr. 8vo. 6s.

THE HEART-SMITER. Second Edition. Cr. 8vo. 6s.

AVENGING CHILDREN. Cr. 8\%o. 6s.

THE PATTEN EXPERIMENT. Medium 8vo. $6 d$.

'THE CEDAR STAR. Medium 8vo. 6d.

Marchmont (A. W.). MISER HOAD. LEY'S SECRET. Medium 8vo. 6\%.

A MOMENT'S ERROR. MTedium 8vo. $6 \pi$.

Marriott (Charles). GENEVRA. Second Edition. Cr. 8zio. 6s.

Marryat (Captain). PETER SIMPI.E Medium 8vo. $6 \%$.

JACOB FAITHFUL. Medium 8vo. $6 \pi$,

Marsh (Ricliard). THE TWICKENHAM PEERAGE. Second Edition. Cr. 8vo. 6s. Also Medium 8ro. $6 d$.

THE MAROUIS OF PUTNEY. Seconat Edition. Cr.8\%o. 6s.

IN THE SERVICE OF LOVE. Third. Edition. Cr.8vo. 6s.

THE GIRL AND THE MIRACLE Third Edition. Cr.8vo. 6s.

THE COWARD BEHIND THE CUR. TAIN. Cr. 8vo. 6s.

THE SURPRISING HUSBAND. Second Edition. Cr.8vo. 6s.

A ROYAL INDISCRETION. Cr. 8vo. 6s.

A METAMORPHOSIS. Medium 8vo. $6 d$.

THE GODDESS. Midium 8vo. $6 d$.

THE JOSS. Medium 8vo. $6 d$.

Marshall (Archibald). MANY JUNES. Second Edition. Cr. 8vo. 6s.

THE SQUIRE'S DAUGHTER. Cr. 8zo. 6s.

Mason (A. E. W.). CLEM E N T N A Illustrated. Third Edition. Cr.8vo. 6s. Also Medium 8vo. 6 .

Mathers(Helen). HON EY. Fourth Ed. Cr. 8vo. 6s. Also Medium 8vo. 6d.
GRIFF OF GRIFFITHSCOURT, Second Edition. Cr. 8vo. $6 s$.

Also Medium 8vo. 6r?

THE FERRYMAN Second Edition. Cr. 8vo. 6s.

TALLY-HO! Fourth Edition. Cr. 8vo. 6s. SAM'S SWEETHEART. Medium 8vo. $6 d$. Maud(Constance). A DAUGHTER OF FRANCE. With a Frontispiece. Second Edition. Cr. 8vo. 6s.

Maxwell(W. B.). VIVIEN. Ninth Edition. Cr. 8vo. 6s.

THE RAGGED MESSENGER. Third Edition. Cr.8vo. 6s.

FABULOUS FANCIES. Cr. 8vo. $6 s$.

THE GUARDED FLAMIE. Seventh Edi. tion. Cr.8vo. 6s.

ODD LENGTHS. Second Ed. Cr. 8vo. 6s. HILL RISE. Fourth Erition. Cr. 8vo. 6s. THE COUNTESS OF MAYBURY: BE. TWEEN YOU AND I. Fourth Edition. Cr. 8ro. 6s.

Meade (L. T.). DRIFT. Second Edition. Cr.8vo. 6s. Also Mredium 8vo. 6r.

RESURGAM. Second Edition. Cr. 8vo. 6s. VICTORY. Cr. 8vo. 6s.

A GIRL OF THE PEOPLE. Illustrated. Fourth Edition. Cr. 8vo. 3 s. 6 \%.

HEPSY GIPSY. Illustrated. Cr. 8vo. 2s. $6 \pi$. THE HONOURABLE MISS : A STORY OF AN OLD-FASHIONED TOWN. Illustrated. Second Edition. Cr. 8vo. 3s. 6d.

Melton (R.). CESAR'S WIFE. Second Edition. Cr. 8io. 6s.

Meredith (Ellis). HEART OF MI HEART. Cr. 8ro. 6s.

Miller (Esther). LIVING LIES. Third Edition. Cr. 8iro. 6s. Also Medium 8vo. $6 d$.

Mitford (Bertram). THE SIGN OF THE SPIDER. Illustrated. Sixth Edition. Cr. 8vo. 3s.6d. Also Medium 8vo. 6d.

IN THE WHIRI OF THE RISING. Third Edition. Cr. 8च0. 6s.

THE RED DERELICT. Second Edition. Cr. 8\%o. 6s.

Molesworth (Mrs.). THE RED GRANGE. Illustrated. Second Edition. Cr. 8ro. $35.6 \pi$.

Montgomery (K. L.). COLONEL KATE. Second Edition. Cr. Svo. 6s.

Montresor (F. F.). THE ALIEN. Thirit Edition. Cr. 3vo. 6s. Also Medium svo. 6 .

Morrison (Arthur). TALES OF MEAN STREETS. Seventis Edition. Cr. 8vo. ós. A CHILD OF THE JAGO. Fifth Edition. Cr. 8vo. 6s.

THE HOLE IN THE. WALL. Fourth Edition. Cr. Svo. 6s. Also Medium 8vo. 6d. 


\section{FICTION}

TO LONDON TOWN. Second Ed. Cr. 8vo. 6s.

DIVERS VANITIES. $C r .8 v 0.6 s$.

Nesbit (E.). (Mrs. H. Bland). THE RED HOUSE. Illustrated. Fourtiz Edition. Cr. 8vo. 6s. Also Medium 8vo. 6d.

Noble (Edward). LORDS OF THE SEA. Second Edition. Cr.8vo. $6 \mathrm{~s}$.

Norris (W. E.). HARRY AND URSULA: A Story with Two Sides to it. Second Edition. Cr. 8wo. $6 s$.

HIS GRACE. Medium 8vo. $6 d$.

GILES INGILBY. Medium 8vo. $6 d$.

THE CREDIT OF THE COUNTY. Mredium 8vo. $6 d$.

LORD LEONARD THE LUCKLESS. Medium 8vo. $6 d$.

MATTHEW AUSTIN. Mredium 8vo. $6 d$. CLARISSA FURIOSA. Medium 8vo. $6 d$.

Oliphant (Mrs.). THE LADY'S WALK. Medium 8vo. $6 d$.

SIR ROBERT'S FORTUNE. Medium 8vo. $6 d$.

THE PRODIGALS. Medium 8vo. $6 d$.

THE TWO MARYS. Medium 8vo. $6 d$.

Ollivant (Alfred). OWD BOB, THE GREY DOG OF KENMUIR. With a Frontispiece. Eleventh Ed. Cr. 8vo. 6s.

Oppenheim (E. Phillips). MASTER OF MEN. Fourth Edition. Cr. 8vo. $6 s$. Also Medizm 8vo. $6 d$.

Oxenham (John). A WEAVER OFWEBS. With 8 Illustrations by MAURICE GREIFFENHAGEN. Fourth Edition. Cr.8vo. $6 s$.

THE GATE OF THE DESERT. With a Frontispiece in Photogravure by HAROLI) Copping. Fifth Edition. $\mathrm{Cr}$. 8vo. $6 \mathrm{~s}$.

PROFIT AND LOSS. With a Frontispiece in photogravure by HAROLD COPPING. Fourth Edition. Cr. 8vio. 6s.

THE LONG ROAD. With a Frontispiece in Photogravure by HAROLD COPPING. Fourth Edition. Cr. 8vo. 6s.

THE SONG OF HYACINTH, AND OTHER STORIES. Second Edition. Cr. 8vo. 6s.

MY LADY OF SHADOWS. Fourt/ Edi. tion. Cr. 8vo. 6s."

Pain (Barry). LINDLEY KAYS. Third Edition. Cr. 8vo. 6s.

Parker (Gilbert). PIERRE AND HIS PEOPLE. Sixth Edition. Cr. 8vo. 6s.

MRS. FALCHION. Fift/ Edition. Cr. 8vo. $6 s$.

THE TRANSLATION OF A SAVAGE. Third Edition. Cr. 8vo. 6s.

THE TRAIL OF THE SWORD. Illustrated. Tenth Edition. Cr. 8vo. 6s. Also Medium 8vo. $6 d$.
WHEN VALMOND CAME TO PONTIAC: The Story of a Lost Napoleon. Sixth Edition. Cr. 8vo. 6s.

Also Medium 8 vo. $6 \%$.

AN ADVENTURER OF THE NORTH. The Last Adventures of 'Pretty Pierre.' Fourth Edition. Cr. 8vo. $6 s$.

THE SEATS OF THE MIGHTY. Illustrated. Sixteenth Edition. Cr. 8rio. 6s.

THE BATTLE OF THE STRONG: a Romance of Two Kingdoms. Illustrated. Sixth Edition. Cr. 8vo. 6s.

THE POMP OF THE LAVILETTES. Third Edition. Cr.8vo. 3s. 6d.

Also Medium 8vo. 6d.

NORTHERN LIGHTS. $C r .8 v o .6 s$.

*Pasture (Mrs. Henry de la). THE TYRANT. Cr. 8vo. $6 s$.

Patterson (J. E.). WATCHERS BY THF SHORE. Second Fdition. Cr. 8vo. 6s.

Pemberton (Max). THE FOOTSTEPS OF A THRONE. Illustrated. Third Edition. Cr. 8ro. 6s.

Also Medium 8vo. $6 \mathrm{~d}$.

I CROWN THEE KING. With Illustrations by Frank Dadd and $A$. Forrestier. Cr. 8vo. $6 s$.

Also Medizım 8vo. $6 d$.

LOVE THE HARVESTER: A STORY oF THE SHIRES. Illustrated. Third Edition. Cr. 8vo. 3s. $6 \pi$.

Phillpotts (Eden). LYING PROPHETS. Third Edition. Cr.8vo. 6s.

CHILDREN OF THE MIST Fifth Edition. Cr. 8vo. $6 s$.

Also Medizm 8vo. $6 \alpha$.

THE HUMAN BOY. With a Frontispiece Sixt/ Edition, Cr. 8vo. $6 s$.

Also Medium 8vo. $6 d$.

SONS OF THE MORNING. Second Edition. Cr. 8vo. 6s.

THE RIVER. Third Edition. Cr. 8vo. 6s. Also Medium 8vo. $6 d$.

THE AMERICAN PRISONER. Fourth Edition. Cr. 8ro. $6 s$.

THE SECRET WOMAN. Fourth Edition. Cr. 8vo. 6s.

KNOCK AT A VENTURE. With a Frontis piece. Third Edition. Cr. 8r.o. 6s.

THE PORTREEVE. Fourth Ed. Cr.8vo. 6s. THE POACHER'S WIFE. Second Fdition. Cr. 8vo. 6s. Also Medium 8vo. 6d.

THE STRIKING HOURS. Second Edition. Crown 8vo. 6s.

THE FOLK AFIELD. Crown 8vo. $6 s$.

Pickthall (Marmaduke). SAID THE FISHERMAN. Seventh Ed. Cr. 8vo. $6 s$. BRENDLE. Second Edition, Cr. 8vo. 6s. THE HOUSE OF ISLAM. Third Edition. Cr. 8vo. $6 s$. 
' $Q$ ' (A. T. Quiller Couch). THE WHI'TE WOLF. Second Fdition. Cr.8wo. 6s. Also Medium 8rio. $6 d$.

THE MAYOR OF TROY. Fourth Edition. Cr. 8vo. 6s.

MFRRY-GARDEN, AND OTHER STORIES. Cr. 8\%o. 65.

MAJOR VICOUREUX. Third Edition. Cr. 8*o. us.

Querido (Israel). TOIL OF MEN. Translated by F. S. ARNOLD. Cr. 8vo. $6 s$.

Rawson (Maud Stepney). THE EN CHANTED GARDLN. liourth Edition. Cr. 8vo. 6s.

THE EASY GO LUCKIES : OR, ONE WAY of LIVING. Second Edition. Cr. Bro. Us. IIAPPINIESS. Cr. 8zo. Gs.

Rhys (Grace). THE WOOING OF SHEILA. Second Fidition. Cr. 8zo. Gs.

TIIE BRIDE. C $r .8 v o$. Gs.

Rislge W. Pett). IOS' PROPERTY. Second Edition. Cr Ero. 6s. Also Medium 8io. 6.t.

ERB. Second Edition. Cr. 8zo. 6s. Also Medium 8r'o. $6 d$.

A SON OF THE STATE. Second Edition. Cr. 8vo. 3s.6d. Also Medium 8ro. 6..

A HREAKER OF IAWS. A Ner Edition Cr. 8vo. 3s. 6d.

MRS. GALFR'S RUSINESS. Illustrated. Seconl Fition. Cr. Sro. 6s.

THE IVICKHAMSES. Fourth Edition. Cr. 8*io. 6s.

NAME OF GARLAND. Third Edition. Cr. 8vo. 6s.

SPLFNDID PROTHFR. Second Edition. Cr. 8r'o. 6s.

GEORGI and THE GENERAL. Medium 8wo. $6 \%$.

Ritchie (Mrs. David G.). MIAN AND 'IIIE CASSOCK. Second Edition. Cr. 8vo. 6s.

Roberts (C. G. D.). THE HEART OF THE ANCIENT WOOD. Cr. 8RO 35.6 .

Robins (Elizabeth). THE CONVFRT. Third Edition. Cr. 8vo. 6s.

Rosenkrantz (Baron Palle). T $\mathrm{H}$ I MIAGISTRATE'S OWN CASE. Cr. 8:0. Gs.

Russell (IV. Clark). MY DANISH S W E E'T H EA R'T. Illustrated. Fifth Edition. Cr. 8vo. 6s. Also Medium 8rio. $6 a^{2}$

IIIS ISLAND PKINCESS. Illustrated. Second Edition. Cr. 8\%o. 6s. Also Medium 8io. 6d.

ABANDONED. Second Edition. Cr. Sio. 6s. Also Medium 8vo. $6 \pi$.

MASTER ROCKAFELLAR'S VOYAGE, Illustrated by Golidon BrownF. Fourth Edition. Cr. 8\%o. $35.6 \%$.
A MARRIAGE AT SEA. Medium 8vo. 6d. Ryan (Marah Ellis). FOR THE SOUL OF RAFAEL. Cr.8vo. 6s.

Sandys (Sydney). JACK CARSTAIRS OF THE: POWER HOUS With 4 Illustrations by Stanley L. Wood. $C r$. 8vo. Gs.

Sergeant (Adeline). THE PASSION OF PAUL MARILLIER. Crozun 8zo. 6s.

THE QUEST OF GEOFFREY D A R R E L L. Cr. $8 z^{\prime} 0.6 s$.

THF, COMING OF THE RANDOLPHS. Cr. 8vo. 6s.

THE PROGRESS OF RACHAEL. $C r$. 8\%o. 6s.

I3ARPARA'S MONEY. MTedium 8wo. 6d. THE MASTER OF BE E CHWOOD. Medium 8:ं0. $6 d$.

THE YELLOW DIAMOND. Second Ed. Cr. 8vo. 6s. Also Mlediame sio. 6u.

"HE LOVF THAT OVERCAME. Medium 8\%o. $6 d$.

Shelley(Bertha). ENDERBY. Third Ed. Cr. 8vo. 6s.

Sidgwick (Mrs. Alfred). THE KINS. MAN. With 8 Illustrations by C. E. Brock. Third Fitition. Cr. 8no. 6s.

THE SEVERINS. Cr. Szo. $6 \mathrm{~s}$.

Smith (Dorothy V. Horace). MISS MONA. Cr. 8:ro. 3s. 6d.

Sonnichsen (Albert). DEEP-SEA VAGA. BONDS. Cr. 8ro. $6 s$.

Stewart (Newton V.). A SON OF THE EMPEROR: BEING PASSAGF FROM THE LIFE OF ENzIO, KING OF SARDINIA AND Corsica. Cr. suo. 6s.

Sunbury (George). THE HA'PENNY MILLIONAIKE. Cr. 8vo. 3s. 6\%.

Surtees (R. S.). HANDLEY CROSS. Iliustrated, Medium $8 \pi 0.61$

MR. SPONGE'S SPORTING TOUR. Illustrated. Medium $8 z^{\prime} 0.6 d$.

ASK NIAMMA. Illus. Medium 8no. 6d.

Swayne (Martin Lutrell). THE BISHOP AND THE LADY. Second Edition. Cr. 8vo. 6s.

Thurston (E. Temple). MIRAGE. Fourth Edition. Cr. Si'o. $6 s$.

Underhill (Evelyn). THE COLUMIN OF DUST. Cr: 8ro. $6 s$.

Urquhart (M.). A TRAGEDY IN COMMIONPLACE. Secord Ed. Cr. 8\%o. 6s.

Vorst (Marie Van). THE SENTIMENTAL ADVENTURES OF JIMIMY BULSTRODE. Cr. 8vo. 6s.

IN AMIBUSH. Cr. 8ro. $6 s$.

Waineman (Paul). T H E B A Y OF L I L A C S : A Romance from Finland. Second Edition. Cr. Sz'0. 6s.

THE SONG OF THE FOREST. $C r .8 * 0$. os. 


\section{FICTION}

Walford (Mrs. L. B.). M R S M I T H. Mediun 8vo. $6 d$.

THE BABY'S GRANDMOTHER. Medium 8vo. $6 d$.

COUSINS. Medium 8vo. 6d.

TROUBLESOME DAUGHTERS. Medium 8vo. $6 d$.

Wallace (General Lew). B E N-H U R. Medium 8vo. 6d.

THE FAIR GOD. Medium 8vo. 6d.

Waltz (Elizabeth C.). THE ANCIENT LANDMARK: A Kentucky Romance. Cr. 8vo. 6s.

Watson (H. B. Marriott). TWISTED EGLANTINE Illustrated. Third Edition. Cr. 8vo. 6s.

THE HIGH TODY : Being further Chapters in the Life and Fortunes of Dick Ryder, otherwise Galloping Dick. With a Frontis. piece. Third Edition. Cr. 8vo. 6s.

A MIDSUMMER DAY'S DREAM. Third Edition. Crown 8vo. 6s.

THE CASTLE BY THE SEA. Second Edition. Cr. 8vo. 6s.

THE PRIVATEERS. Illustrated. Second Edition. Cr. 8vo. 6s.

A POPPY SHOW : Being Divers and Diverse Tales. Cr. 8vo: $6 s$.

THE FLOWER OF THE HEART. Third Edition. Cr. 8vo. 6s.

THE ADVHNTURERS. Medium 8vo. 62.

Webling (Peggy). THE STORY OF VIRGINIA PERFECT. Third Edition. Cr. 8vo. 6s.

Weekes (A. B.). THE PRISONERS OF WAR. Medium 8vo. $6 \pi$.

Wells (H. G.). THE SEA LADY. Cr. 8vo. 6s. Also Mediwn 8vo. 6d.

Weyman (Stanley). UNDER THE RED ROBE. With Illustrations by R. C. WOODVILLE. Twenty-Second Ed. Cr.8vo. $6 s$.

Whitby (Beatrice). THE RESULT OF AN ACCIDENT. Second Edition. Cr. svo. $6 s$.

White (Percy). THE SYSTEM. Third Edition. Cr. 8vo. 6 s.
A PASSIONATE PILGRIM. Medium Sขo. 6 ?.

LOVE AND THE WISE MEN. Cr. 8vo. $6 s$.

Williams (Margery). THE BAR. Cr. 8vo. 6s.

Williamson (Mrs. C. N.). TH E A DVENTURE OF PRINCESS SYLVIA. Second Edition. Cr. 8vo. 6s.

THE WOMAN WHO DARED. Cr. 8vo. $6 s$.

THE SEA COULD TELL. Second Edition. Cr. 8vo. 6s.

THE CASTLE OF THE SHADOWS. Third Edition. Cr. 8vo. 6s.

PAPA. Cr. 8vo. 6s.

Williamson (C. N. and A. M.). THE LIGHTNING CONDUCTOR: The Strange Adventures of a Motor Car. With I6 Illustrations. Seventeenth Edition. Cr. 8vo. 6s. Also Cr. 8vo. is. net.

THE PRINCESS PASSES: A Romance of a Motor. With I 6 Illustrations. Ninth Edition. Cr. 8vo. 6s.

MY FRIEND THE CHAUFFEUR. With x6 Illustrations. Tenth Edit. Cr.8vo. $6 s$.

LADY BETTY ACROSS THE WATER. Tenth Edition. Cr. 8vo. 6 s.

THE CAR OF DESTINY AND ITS ERRAND IN SPAIN. With I7 Illustrations. Fourth Edition. Cr. svo. 6s.

THE BOTOR CHAPERON. With a Frontispiece in Colour by A. H. BUCKLAND, 16 other Illustrations, and a Map. Fifth Edition. Cr. 8vo. $6 s$.

SCARLET RUNNER. With a Frontispicce in Colour by A. H. BUCKLAND, and 8 other Illustrations. Third Ed. Cr. 8vo. $6 s$.

SET IN SILVER. With a Frontispiece. Sccond Edition. Cr. 8vo. 6s.

Wyllarde (Dolf). THE PATHTVAY OF THE PIONEER (Nous Autres). Fourth Edition. Cr. 8vo. 6s.

Yeldham (c. C.). DURHAM'S FARM. Cr. 8vo. 6s.

\section{Books for Boys and Girls}

\section{Illustrated. Crown 8vo. 3s. 6a.}

The, GetTing Well of Dorothy. By Mrs. W. K. Clifford. Second Edition.

Only a Guard-Room Dog. By Edith E. Cuthell.

Master Rockafrllar's Voyage. By WV. Clark Russell. Fourth Edition.

SyD Belton: Or, the Boy who would not go to Sea. By G. Manville Fenn. Second Ed.
The Red Grange. By Mrs. Molesworth.

A Girl of the People. By L. T. Meade. Fourth Edition.

Hepsy Gipsy. By L. T. Meade. 2s. 6it. The Honourable Miss. By L. T. Meade. Second Fition.

There was oncei a Prince. By Mrs. M. li. Mann.

When Arnold comes Home. Ty Mrs. M. L. Manu. 


\section{The Novels of Alexandre Dumas}

\section{Medium 8vo. Price 6d. Double Volumes, is.}

Acté.

The Adventures of Captain Pamphile.

AMAURY.

THE BIRD OF FATE.

The Black Tulip.

The Castle of Eppstein.

Catherine Blum.

CECILE.

The Chevalier D'Harmental. (Double volume.) is.

Chicot The Jester.

CONSCIENCE.

'THE CONVICT's Son.

The Corsican Brothers: and Otho the ARCHER.

Crop.EAREd JacQuot.

DOM GORENFLOT.

The Fatal Combat.

The Fencing Master.

FERNANDE.

Gabriel Lambert.

Georges.

The Great Massacre.

HENRI DE NAVARRE.
Hélène de Chaverny.

THE HOROSCOPE.

LOUISE DE LA VAllikere. (Double volume.)

IS.
THE MAN IN THE IRON MASK. (Double volume.) Is.

Maitize Adam.

'The Mouth of Hell.

NANON. (Double volume.) is.

Pauline; Pascal Bruno; and Bontekoe.

PÈrE la RUINE.

The Prince of Thieves.

THE REMINISCENCES OF ANTONy.

ROBIN HOOD.

The Snowball and Sultanetta.

SYI,VANDIRE.

TAles of THE SUPERnatural.

TAles of STrange AdVENTURE.

The Three Musketeers. (Double volume.) Is.

THE TRAGEDY OF NANTIS.

TwENTY Years AFTER. (Double volume.) Is. TIIE WILD-DUCK Shooter.

THE WOLF-LEADER.

\section{Methuen's Sixpenny Books}

\section{Mediun Svo.} Albanesi (E. Maria). L O V IE AN D Brownell (C. L.). THE HEART OF
LOUISA. I KNOW A MAIDEN.

Anstey (F.). A BAYARD OF BENGAL. Austen(J.). PRIDE AND PREJUDICl: Bagot (Richard). A ROMAN MYSTERI. CASTING OF NITS.

DONNA DIANA.

Balfour (Andrew). BY STROKE OF SWORD.

Baring=Gould (S.). FURZE BLOOM.

CHEAP JACK ZITA.

KITTY ALONE.

URITH.

THE BROOM SQUIRE.

IN THE ROAR OF THE SEA.

NOEMI.

A BOOK OF FAIRY TALES. Illustrated. LITTLE TU'PENNY.

WINEFRED.

THE FROBISHERS.

THE OUEEN OF LOVE.

ARMINELL.

Barr (Robert). JENNIE BAXTER.

IN THE MIDST OF ALARMS.

THE COUNTESS TEKIA.

THE MUTABLE MANY.

Benson (E. F.). DODO.

'THE VINTAGI.

Burton (J. Bloundelle). ACROSS THE SALT SEAS.

Caffyn (Mrs.). ANNE MAULEVERER. Capes (Bernard). THE LAKE OF IVINE.

Clifford (Mrs. W. K.). A FLASH OF SUMIMER.

MRS. KEITH'S CRIME.

Corbett (Julian). A BUSINESS IN GREAT WATERS.

Croker (Mrs. B. M.). ANGEL.

A STATE SECRET.

PEGGY OF THE BARTONS.

JOHANNA.

Dante (Alighieri). THE D I VI NE COMEDY (Cary).

Doyle (A. Conan). ROUNI THE RED LAMP.

Duncan (Sara Jeannette). A VOYAGE OF CONSOLATION.

THOSE DELIGHTFUL AMERICANS.

Eliot (George). THE $1 I I L L$ ON THE FLOSS.

Findlater (Jane H.). THE GREEN GRAVES OF BALGOWRIE.

Gallon (Tom). RICKERBY'S HOLLY.

(iaskell (Mrs.). CRANFORD.

MARY BAR'ION.

Brontē (Charlotte). SHIRLEY. NORTH AND SOUTH. 
Gerard (Dorothea). HOLY MATRI- Montresor (F. F.). THE ALIEN.

MIONY.

MADE OF MONEY.

Gissing (G). THE TOWN TRAVELLER. THE CROWN OF LIFE.

Glanville (Ernest). THE I NCA'S TREASURE.

THE KLOOF BRIDE.

Gleig (Charles). BUNTER'S CRUISE.

Grimm (The Brothers). GRIMM'S FAIRY TALES.

Hope (Anthony). A MAN OF MARK.

A CHANGE OF AIR.

THE CHRONICLES OF COUN'T ANTONIO.

PHROSO.

THE DOLLY DIALOGUES.

Hornung (E. W.). DEAD MEN TELL NO TALES.

Ingraham (J. H.). THE THRONE OF DAVID.

Le Oueux (W.). THE HUNCHBACK OF IVESTMINSTER.

Levett-Yeats (S. K.). THE TRAITOR'S WAY.

ORRAIN.

Linton (E. Lynn). THE TRUE HIS. TORY OF JOSHUA DAVIDSON.

Lyall (Edna). DERRICK VAUGHAN.

Malet (Lucas). THE CARISSIMA.

A COUNSEL OF PERFECTION.

Mann (Mrs. M. E.). MRS. PETER HOWARD.

A LOST ESTATE.

THE CEDAR STAR.

ONE ANOTHER'S BURDENS.

THE PATTEN EXPERIMENT

A WINTER'S TALE.

Marchmont (A. W.). MIISER HOAD. LEY'S SECRET

A MONENT'S ERROR.

Marryat (Captain). PETER SIMPLE. JACOB FAITHFUL.

Marsh (Richard). A METAMORPHOSIS. THE TWICKENHAM PEERAGE. THE GODDESS.

THE JOSS.

Mason (A. E. W.). CLEMENTINA.

Mathers (Helen). HONEY.

GRIFF OF GRIFFITHSCOURT,

SAM'S SWEETHEART.

Meade (Mrs. L. T.). DRIFT.

Miller(Esther). LIVING LIES.

Mitford (Bertram). THE SIGN OF THE Morrison (Arthur). THE HOLE IN THE WALL.

Nesbit (E.) THE RED HOUSE.

Norris (W. E.). HIS GRACE.

GILES INGILBY.

THE CREDIT OF THE COUNTY.

LORD LEONARD THE LUCKLESS.

MATTHEIV AUSTIN.

CLARISSA FURIOSA.

Oliphant (Mrs.). THE LADY'S WALK.

SIR ROBERT'S FORTUNE.

THE PRODIGALS.

THE TWO MARYS.

Oppenheim (E. P.). MASTER OF MEN.

Parker (Gilbert). THE POMP OF THE LAVILETTES.

WHEN VALMOND CAME TO PONTIAC THE TRAIL OF THE SWORD.

Pemberton (Max). THE FOOTSTEPS OF A THRONE.

I CROWN THEE KING.

Phillpotts (Eden). THE HUMAN BOY. CHILDREN OF THE MIST.

THE POACHER'S IVIFE.

THE RIVER.

' $Q$ ' (A. T. Quiller Couch). TH E WHITE WOLF.

Ridge(W. Pett). A SON OF THE STATE. LOST PROPERTY.

GEORGE and THE GENERAL.

ERB.

Russell (W. Clark). ABANDONED.

A MARRIAGE AT SEA.

MY DANISH SIVEETHEART.

HIS ISLAND PRINCESS.

Sergeant (Adeline). THE MASTER OF BEECHWOOD.

BARBARA'S MIONEY.

THE YELIOW DIAMOND.

THE LOVE THAT OVERCAME.

Sidgwick (Mrs. Alfred). THE KINS. MAN.

Surtees (R. S.). HANDLEY CROSS. IIR. SPONGE'S SPORTING TOUR. ASK MAMMA.

Walford (Mrs. L. B.). MIR. SMITH. COUSINS.

THE BABY'S GRANDMOTHER.

TROUBLESOME DAUGHTERS.

Wallace (General Lew). BEN.HUR.

THE FAIR GOD.

Watson (H. B. Marriott). THE ADVEN TURERS.

Weekes (A. B.). PRISONERS OF WAR.

Wells (H. G.). 'THE SEA LADY.

White (Percy). A PASSIONATE PILGRIM. 




$$
53771
$$


